AUTOMATING IMAGE-BASED

CADASTRAL BOUNDARY MAPPING

Sophie Charlotte Crommelinck 



\title{
AUTOMATING IMAGE-BASED CADASTRAL BOUNDARY MAPPING
}

\author{
DISSERTATION
}

to obtain

the degree of doctor at the University of Twente, on the authority of the rector magnificus, prof.dr. T.T.M. Palstra

on account of the decision of the Doctorate Board, to be publicly defended

on Friday $25^{\text {th }}$ October 2019 at 12.45

by

Sophie Charlotte Crommelinck

born on $08 / 05 / 1992$

in Cologne, Germany 
This thesis has been approved by

Prof.dr.ir. M.G. Vosselman, supervisor

Dr.-Ing. Y. Yang, co-supervisor

Dr. M.N. Koeva, co-supervisor

ITC dissertation number 368

ITC, P.O. Box 217, 7500 AE Enschede, The Netherlands

ISBN 978-90-365-4874-8

DOI 10.3990/1.9789036548748

Cover designed by J ob Duim

Printed by ITC Printing Department

Copyright (c 2019 by Sophie Crommelinck

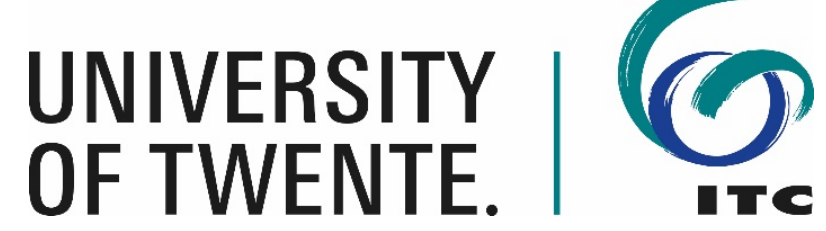


Graduation committee:

\section{Chairman/ Secretary}

Prof.dr.ir. A. Veldkamp University of Twente (ITC)

\section{Supervisor}

Prof.dr.ir. M.G. Vosselman University of Twente (ITC)

\section{Co-supervisors}

Dr.-Ing. Y. Yang

Dr. M.N. Koeva

University of Twente (ITC)

University of Twente (ITC)

\section{Members}

Prof.dr.ir. J.A. Zevenbergen

University of Twente (ITC)

Prof.dr.ir C.H.J. Lemmen

Prof. Dr. B. Höfle

Prof. Dr.-Ing. N. Haala

University of Twente (ITC)

University of Heidelberg

University of Stuttgart 



\section{Acknowledgements}

This work was supported by the Horizon 2020 program of the European Union [project number 687828].

I would like to thank my supervisors who have supported and guided this work. George, who was able to continuously identify and understand my work's bottlenecks. You taught me to pay attention to the precise wording of my findings and to keep up a critical attitude towards them. Mila, who often insisted on setting my work in an applied context, teaching me to never forget the end user and to value personal communication in a research team. Michael, who provided precious insights from computer vision. You helped me to improve the contextualization of my work from a technical research perspective.

I would also like to thank Rohan and Markus who supervised me in the first year(s) of my Ph.D. Rohan added a practical dimension to my work and continued to impress me with his writing skills, as well as with his eagerness and competence to put all its4land pieces together. You taught me the value of taking in different perspectives and to ask simple questions. Markus, who always managed to understand my methodological ideas and struggles, advising me to break down a problem in manageable chunks. I want to thank Bernhard, who supervised me during my master and my research stay in Heidelberg. It is due to his guidance that I have learned the basics of solid research work, the value of patience in the research process, and the importance to finish one step properly before continuing the research process.

Thank you my colleagues at ITC. I enjoyed being part of such an international and diverse working environment. The social events we shared were always worthwhile. Thank you Claudia, with whom I shared an office and many thoughts about on-going work and life. Overall, this international setting in Enschede allowed me to improve my Dutch and my English for which I am thankful.

Thank you its4land team for providing an international and multi-disciplinary context to this Ph.D. work. Our regular its4land and tech4land meetings, which we documented on share4land, thought me to handle the challenge of reaching consensus and progress with a diverse team through online and offline communication. You helped me to take off the scientific glasses once in a while and focus on aligning varied goals and motivations.

Thank you my athletic friends for endless hours of running, cycling, and finessing. Together with you, I have matured into a mentally and physically fitter runner. Thank you LAAC group for a solid track workout every Thursday. 
Thanks to my engelhorn sports teammates for keeping me as a long-distance member and always welcoming me back at events around Heidelberg.

Thank you Maphie for keeping up our long-distance friendship through skyping and traveling together.

Thanks to my family for always being there for me and supporting me in whatever I decide to do. Thanks to my two uncles J an and Herman for helping me with the Dutch summary of this thesis.

From all the discoveries I made throughout this Ph.D., Pierre is the one I am most grateful for. Thank you Pierre for being with me and supporting my every stride. 


\section{Table of Contents}



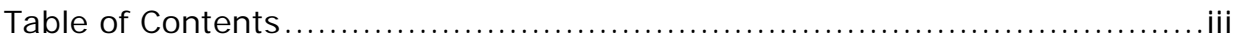

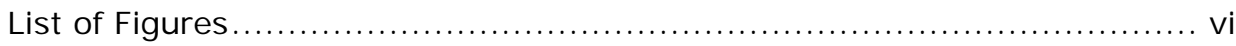



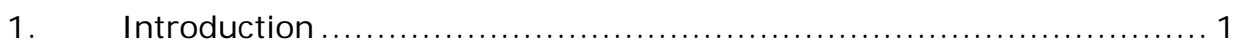

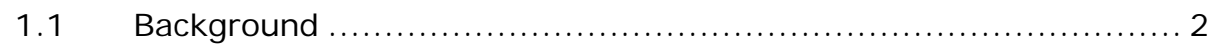





1.1.3 Application of UAV-based Cadastral Mapping.................. 4

1.1.4 Boundary Delineation for UAV-based Cadastral Mapping....... 5

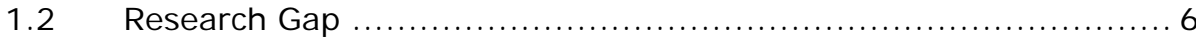

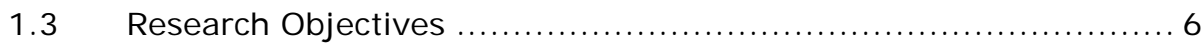



2. Review of Automatic Feature Extraction from High-Resolution Optical

Sensor Data for UAV-based Cadastral Mapping .................................. 9





2.1.1 Objective and Organization of the Study .................. 11

2.2 Review of Feature Extraction and Evaluation Methods .............. 13

2.2.1 Cadastral Boundary Characteristics........................ 13

2.2.2 Feature Extraction Methods .............................. 15

2.2.3 Accuracy Assessment Methods ........................... 26

2.3 Discussion .............................................................. 28

2.3.1 Cadastral Boundary Characteristics....................... 28

2.3.2 Feature Extraction Methods ............................. 28

2.3.3 Accuracy Assessment Methods .............................. 31

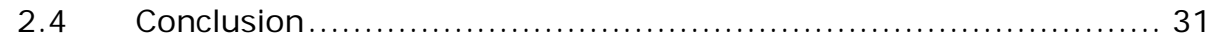

3. Contour Detection for UAV-based Cadastral Mapping ................... 35

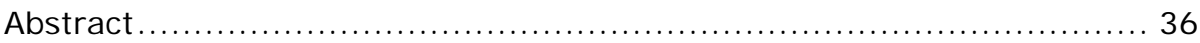



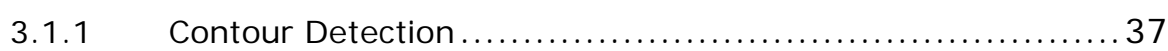

3.1.2 Objective and Organization of the Study .................... 39

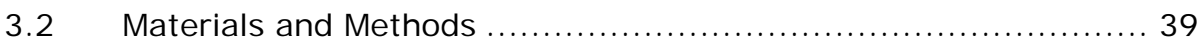

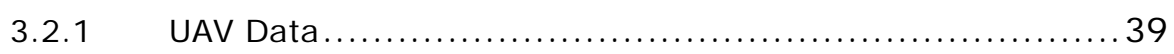

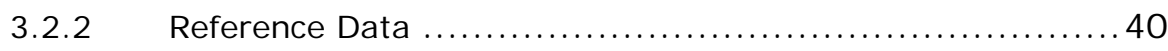

3.2.3 Image Processing Workflow .............................. 41

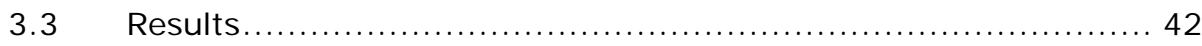

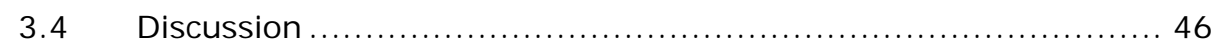

3.4.1 Detection Quality ........................................ 46

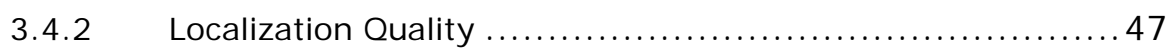


3.4.3 Discussion of the Evaluation Approach..................... 47

3.4.4 Transferability and Applicability of gPb for Boundary

Delineation............................................. 48

3.5 Conclusion............................................................ 49

4. SLIC Superpixels for Object Delineation from UAV Data ................ 51

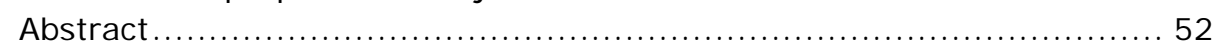

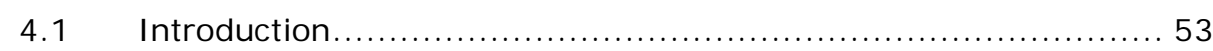

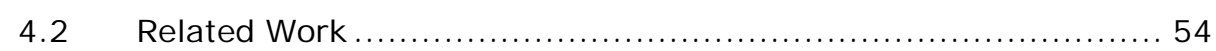

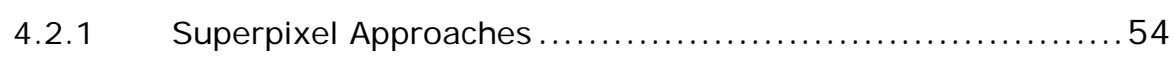

4.2 .2 SLIC Approach ........................................ 54

4.2.3 Superpixels in Remote Sensing.......................... 56

4.2.4 SLIC Superpixels for Object Delineation ..................... 57

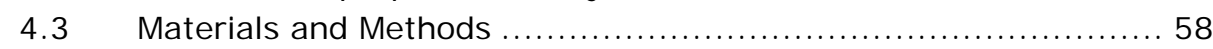

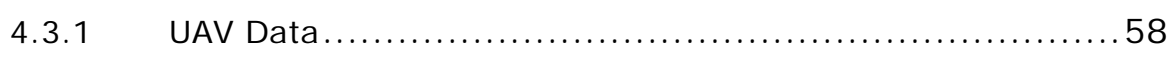



4.3.3 Image Processing Workflow ............................ 59

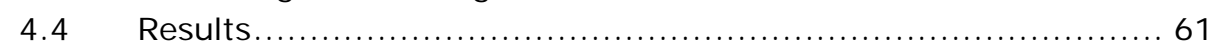

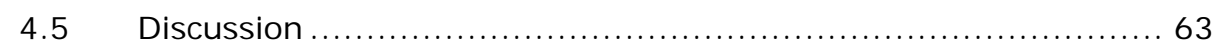

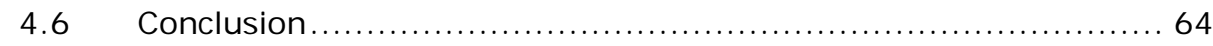

5. Interactive Boundary Delineation from UAV Data .....................6 67

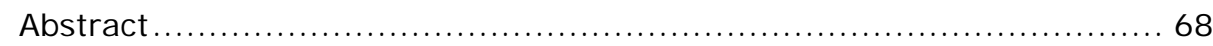

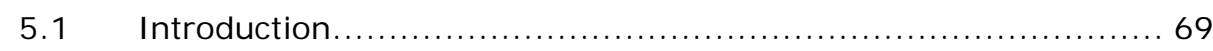

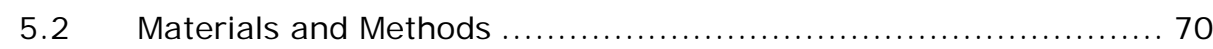



5.2.2 Image Processing Workflow ............................... 71

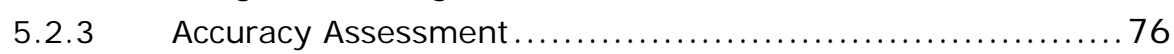

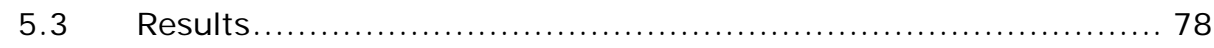

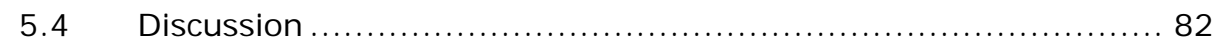

5.5 Conclusion.............................................................. 83

6. Validating and Improving Automated Feature Extraction for UAV-based

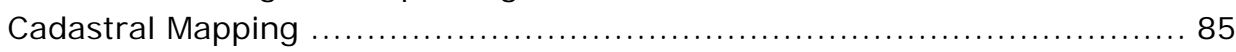

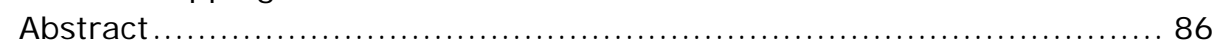

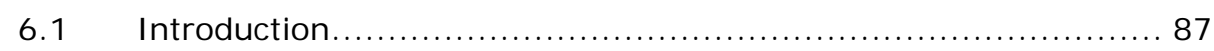

$6.2 \quad$ Materials and Methods ............................................... 87



6.2.2 Boundary Delineation Approach .......................... 90

$6.2 .3 \quad$ Accuracy Analysis ......................................... 93

6.2.4 Operational Analysis ...................................... 97

$6.2 .5 \quad$ Feedback Analysis ....................................... 98

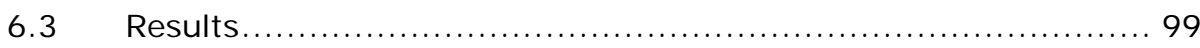

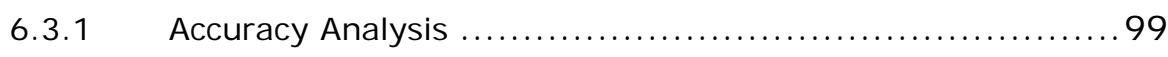

6.3.2 Operational Analysis ................................... 103 
6.3.3 Feedback Analysis .................................. 108

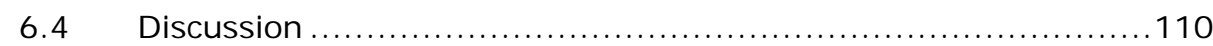



7. Deep Learning for Boundary Line Classification in Cadastral Mapping 115

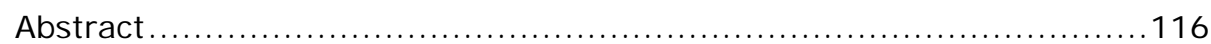

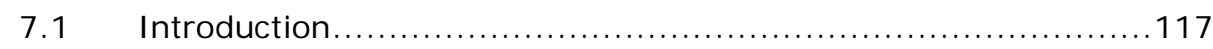

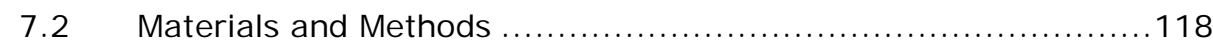

7.2.1 Image Data ........................................ 118

7.2.2 Boundary Mapping Approach........................... 119

7.2.3 Accuracy Assessment................................. 123

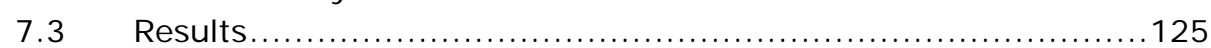

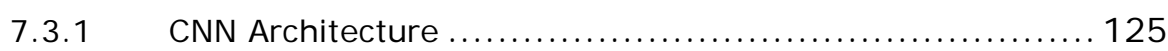

7.3.2 RF vs. CNN Classification ............................. 127

7.3.3 Manual vs. Automated Delineation....................... 128

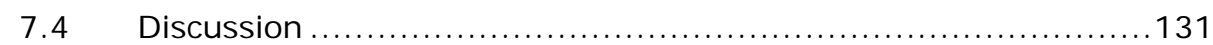

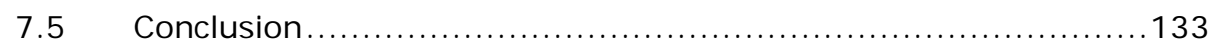

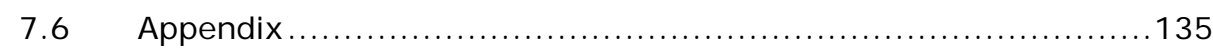

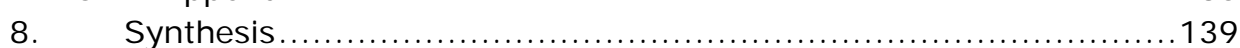

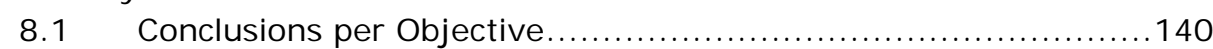

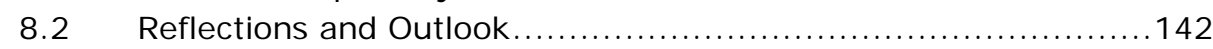

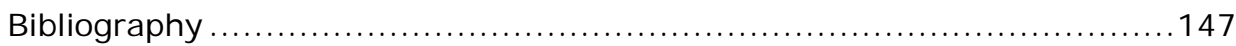

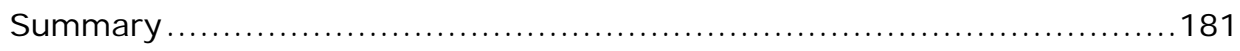

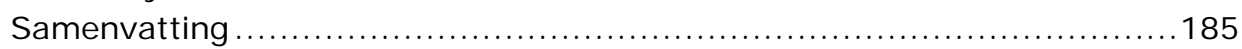

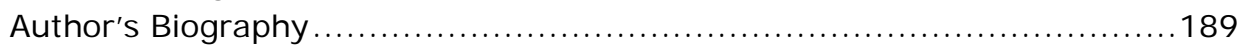




\section{List of Figures}

Figure 1.1. Structure of its 4 land project. This Ph.D. research is based on one of the four technical work packages, namely Automate It. ................ 3

Figure 2.1. Overview of cadastral surveying techniques and cadastral boundary concepts that contextualize the scope of this review paper. The lines between different categories are fuzzy and should not be understood exclusively. They are drawn to give a general overview.

Figure 2.2. Characteristics of cadastral boundaries extracted from highresolution optical remote sensors. The cadastral boundaries are derived based on (a) roads, power lines and pipelines [38], (b) fences and hedges [10], (c/ d) crop types [31], (e) roads, foot paths, water drainage, open areas and scrubs [75], and (f) adjacent vegetation [71]. (d) Shows the case of a nonlinear irregular boundary shape. The cadastral boundaries in (e) and (f) are often obscured by tree canopy. Cadastral boundaries in (ad) are derived from UAV data; in (e) and (f) from HRSI. All of the boundaries are manually extracted and delineated...................... 15

Figure 2.3. Pixel-based and object-based feature extraction approaches aim to derive low-level and high-level features from images. Object-based approaches may include information provided by low-level features that is used for high-level feature extraction.

Figure 2.4. Sequence of commonly applied workflow steps to detect and extract linear features used to structure the methods reviewed. ....... 17

Figure 2.5. Spatial resolution of data used in the case studies. The figure shows the 52 case studies, in which the spatial resolution was known. For case studies that use datasets of multiple resolutions, the median resolution is used. For 37 further case studies, which are not represented in the histogram, the spatial resolution was left undetermined. 18

Figure 2.6. UAV-derived orthoimage that shows a rural residential housing area in Namibia, which is used as an exemplary dataset to implement representative feature extraction methods. 19

Figure 2.7. (a) Subset of the original UAV orthoimage converted to greyscale. (b) Anisotropic diffusion applied to greyscale UAV image to reduce noise. After filtering, the image appears smoothed with sharp contours removed, which can be observed at the rooftops and tree contours............... 19

Figure 2.8. I mage segmentation applied to the original UAV orthoimage: (a) graph-based segmentation, (b) SLIC segmentation, and (c) Watershed segmentation. The label matrices are converted to colors for visualization purpose. The input parameters are tuned to obtain a comparable number of segments from each segmentation approach. However, all approaches result in differently located and shaped segments.

Figure 2.9. Edge detection applied to the greyscale UAV orthoimage based on (a) Canny edge detection and (b) the Laplacian of Gaussian. The output is a binary image in which one value represents edges (green) and the other value represents the background (black). (c) Shows the line segment detector applied and imposed on the original UAV orthoimage. 
Figure 2.10. (a) Douglas-Peucker simplification (red) of the contour generated with snakes (green). The simplified contour approximates the fence that marks the cadastral boundary better than the snake contour does. (b) Binary image derived from Canny edge detection as shown in Figure 2.9a. The image serves as a basis for morphological closing, shown in (c). Through dilation followed by erosion, edge pixels (green) belonging to one class in (b) are connected to larger regions in (c).... 26

Figure 3.1. Combined $\mathrm{gPb}$ contour detection and hierarchical image segmentation for the delineation of closed object contours from RGB images described in [116]. The example image is taken from the 'Berkeley Segmentation Dataset and Benchmark' [247] and is processed with the publicly available source code for gPb contour detection [248]. ......... 38

Figure 3.2. Manually delineated object contours used as reference data to determine the detection quality overlaid on UAV orthoimages of (a) Amtsvenn, Germany, (b) Toulouse, France, and (c) Lunyuk, Indonesia.

Figure 3.3. Image processing workflow for delineation of visual object contours from UAV orthoimages and its assessment based on the

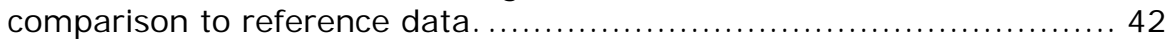

Figure 3.4. Examples of contour maps $(\mathbf{a}, \mathbf{d}, \mathbf{g})$ and binary boundary maps $(k=0.1)$ of Amtsvenn $(\mathbf{a}, \mathbf{b}, \mathbf{c})$, Toulouse $(\mathbf{d}, \mathbf{e}, \mathbf{f})$ and Lunyuk $(\mathbf{g}, \mathbf{h}, \mathbf{i})$. The boundary maps are buffered with $2 \mathrm{~m}$ to increase their visibility. (a, $\mathbf{b}, \mathbf{d}, \mathbf{e}, \mathbf{g}, \mathbf{h})$ result from an untiled input image of $1000 \times 1000$ pixels, $(\mathbf{c}, \mathbf{f}, \mathbf{i})$ from an input image of $5000 \times 5000$ pixels merged from 25 tiles.

Figure 3.5. Binary boundary maps derived from untiled UAV orthoimage of Amtsvenn with a size of $1000 \times 1000$ pixels and $100 \mathrm{~cm}$ GSD at level (a) $k=0.1$, (b) $k=0.3$, and (c) $k=0.5$.

Figure 3.6. Detection quality: the errors of commission and omission is shown for binary boundary maps of different Ground Sample Distances (GSD) derived for (a) Amtsvenn, (b) Toulouse, and (c) Lunyuk at level k = 0.1.

Figure 3.7. Localization quality: the distance between pixels being True Positives (TP) and the reference data relative to the total number of TPS per Ground Sample Distance (GSD) is shown for (a) Amtsvenn, (b) Toulouse, and (c) Lunyuk at level $\mathrm{k}=0.1$.

Figure 4.1. (a) SLIC $(m=20)$ and (b) SLICO applied to an UAV orthoimage of Toulouse with $0.05 \mathrm{~m}$ ground sample distance (GSD) and $\mathrm{k}=625$. SLIC generates regular-shaped superpixels in untextured regions and highly irregular superpixels in textured regions. SLICO generates regular-shaped superpixels across the scene, regardless of texture. SLICO superpixels are spatially more compact, but spectrally more heterogeneous............ 56

Figure 4.2. Manually delineated outlines of exactly localizable roads and roofs used for the accuracy assessment overlaid on UAV orthoimages of (a) Amtsvenn in Germany and (b) Toulouse in France. Outlines in close spatial proximity, such as two parallel outlines of roads, might appear as a thicker line, as they consist of two parallel lines in the reference data. 
Figure 4.3. SLIC outlines derived for compactness parameters $(\mathbf{a}, \mathbf{b}) \mathrm{m}=1$, (c, d) $m=20$, and $(\mathbf{e}, \mathbf{f})$ SLICO, where $m$ is adaptively refined for each superpixel. The first row of images shows superpixels overlaid on the orthoimage of Amtsvenn, while the second row shows superpixels overlaid on the orthoimage of Toulouse, both for $k=10,000$. .. 61

Figure 4.4. Errors of omission obtained for (a) Amtsvenn and (b) Toulouse. The number of superpixels $k$ varies according to the extent covered per study area (Table 4.2).

Figure 4.5. Errors of commission obtained for (a) Amtsvenn and (b) Toulouse. The number of superpixels $k$ varies according to the extent covered per study area (Table 4.2).

Figure 5.1. Sequence of a commonly applied workflow proposed in [44]. The workflow aims to extract physical objects related to those manifesting cadastral boundaries from high-resolution optical sensor data. For the first and second component, state-of-the-art computer vision approaches have been evaluated separately and determined as efficient for UAV-based cadastral mapping [240,261]. The third component as well as the overall approach is described in this paper. .. 70

Figure 5.2. UAV data from (a) Amtsvenn and (b) Gerleve overlaid with SLIC lines used for training (30\%) and validation (70\%).... 71

Figure 5.3. Workflow of globalized probability of boundary (gPb) contour detection and hierarchical image segmentation resulting in a binary boundary map containing closed boundaries. 72

Figure 5.4. Workflow of simple linear iterative clustering (SLIC) resulting in agglomerated groups of pixels, i.e., superpixels, whose boundaries outline physical objects in the image.

Figure 5.5. Workflow of interactive delineation: each superpixel outline is split, wherever outlines of three or more adjacent superpixels have a point in common (visualized by line color). Attributes are calculated per line. They are used by a RF classifier to predict boundary likelihoods (visualized by line thickness). User-selected nodes (red points) are connected along the lines of highest likelihoods. 76

Figure 5.6. (a) Detection quality, for which delineation data are buffered with $0.05 \mathrm{~m}$ and reference data with $0.2 \mathrm{~m}$. Both are overlaid to calculate the number of pixels being TP, FN, TN or FP. (b) Localization quality, for which the reference data are buffered with 0.05-0.2 $\mathrm{m}$ and overlaid with the buffered delineation data to calculate the sum of TPs per buffer distance.

Figure 5.7. Classification performance: localization quality for SLIC lines of different cost values $\mathrm{c}$ assigned through the RF classification for (a) Amtsvenn and (b) Gerleve. 79

Figure 5.8. Examples of the interactive delineation (green) along SLIC lines (red). The thicker a SLIC line, the lower c.......................... 81

Figure 5.9. Interactive outlining performance: localization quality for delineation for (a) Amtsvenn and (b) Gerleve. Both the reference and the interactively delineated data consists of lines that are rasterized to quantify the localization quality. 
Figure 6.1. UAV data tiles of $250 \times 250 \mathrm{~m}$ and a $5 \mathrm{~cm}$ GSD of Muhoza (a-c)

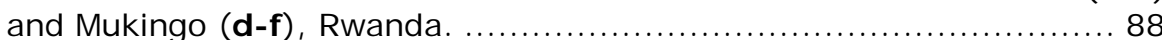

Figure 6.2. UAV data tiles of $250 \times 250 \mathrm{~m}$ and a $6 \mathrm{~cm}$ GSD of Kajiado, Kenya.

Figure 6.3. UAV data tiles of $250 \times 250 \mathrm{~m}$ and $150 \times 150 \mathrm{~m}$ and a $6 \mathrm{~cm}$ GSD of Mailua, Kenya.

Figure 6.4. Boundary mapping approach: (a) MCG image segmentation. (b) Boundary classification that requires line labeling into 'boundary' and 'not boundary' for training. The labeled lines are used together with line-based features to train a Random Forest classifier that generates boundary likelihoods for testing. (c) Interactive delineation guided by a QGIS plugin that creates a least-cost-path between user-selected nodes along simplified lines from (a) with highest boundary likelihoods generated in (b).

Figure 6.5. Spatial correctness based on overlaying the buffered delineation and reference data to compute pixels being True Positive (TP) or False Positive (FP). These pixels are then summated to calculate (a) the error commission and (b) the correctness. (c) We implemented the described procedure for line-based accuracy assessment as a new 'LineComparison' QGIS plugin.

Figure 6.7. (a) Explaining concepts of the boundary mapping approach during the workshop introduction. (b) Demonstrating the boundary mapping approach during the interactive feedback session. (c) Discussing strengths, weaknesses, opportunities, and threats (SWOT) of the approach proposed. 99

Figure 6.8. Examples of delineation results for Rwandan study areas: (a/ b) Building delineation. (d/ e) Parcel delineation. (c) Building segmentation requiring editing. (f) Building segmentation from different resolutions. (g) Visible parcels not demarcated by objects but by context. (h) Wall outline and centerline used for parcel delineation. (i) Boundary classification trained to detect buildings (top) and parcels (bottom). 102

Figure 6.9. Boundary mapping in Mailua: image segmentation, boundary classification, and interactive delineation applied to delineate visible boundaries of pastoralists' homesteads from UAV data. .104

Figure 6.10. (a-d) Examples of visible boundaries in Kajiado. (e-h) Boundary demarcations challenging to identify correctly from remote sensing imagery 105

Figure 6.11. (a/ b) Cadastral boundaries delineated from UAV data with the proposed boundary mapping approach. 107

Figure 6.12. Challenges observed during delineation: (a) undersegmentation, (b) over-segmentation, (c) fragmented segmentation, (d) redundancy of least-cost-path calculation, (e) visible boundary not demarcated by objects, but by context, and (f) identification of delineation areas through boundary mapping approach. 107

Figure 6.13. Transferring an innovative idea to a successful application along technology readiness levels (TRL) proposed by the European Commission [331]. .108 
Figure 6.14. SWOT results considering technology readiness levels (TRL) (a) 1-3, (b) 4-6, and (c) 7-9.

Figure 6.15. From physical object to cadastral boundary: reformulated boundary concepts for indirect surveying.

Figure 7.1. Boundary Delineation workflow proposed to improve indirect surveying. This study optimizes image segmentation, questions whether Random Forest (RF) or Convolutional Neural Networks (CNN) are better suited to derive boundary likelihoods for visible object outlines, and introduces additional functionalities for the interactive delineation. ...117

Figure 7.2. (a) Aerial image of $0.25 \mathrm{~m}$ GSD for a rural scene in Ethiopia, divided into areas for training and testing our approach before comparing results to (b) cadastral reference. UAV images for peri-urban scenes in (c) Rwanda (0.02 m GSD), and (d) Kenya (0.06 m GSD) to compare automated to manual delineation.

Figure 7.3. MCG image segmentation lines around visible objects before and after simplification reducing the line count by $80 \%$

Figure 7.4: Boundary line classification based on Random Forest (RF) to derive boundary likelihoods for MCG lines. 121

Figure 7.5. Boundary line classification based on Convolutional Neural Networks (CNN) to derive boundary likelihoods for MCG lines..........122

Figure 7.6: Interactive delineation functionalities: (a) connect lines surrounding a click, or (b) a selection of lines. (c) Close endpoints of selected lines to a polygon. (d) Connect lines along least-cost-path. ...123

Figure 7.7. Interface of open source QGIS BoundaryDelineation plugin [306] developed to guide interactive delineation functionalities. ............... 123

Figure 7.8. Accuracy and loss for our fine-tuned VGG19................. 127

Figure 7.9. (a) Automated delineation requires clicking once somewhere in the parcel, while manual delineation requires precise clicking at least four times on each corner. (b) Boundaries partly covered or delineated by vegetation impede indirect surveying and limit the effectiveness of our automated delineation compared to manual delineation. 


\section{List of Tables}

Table 2.1. Case study examples for image segmentation methods.......... 22

Table 2.2. Case study examples for line extraction methods................. 23

Table 2.3. Case study examples for contour generation methods........... 24

Table 2.4. Case study examples for post-processing methods. .............. 25

Table 3.1. Specifications of UAV datasets per study area. .................... 40

Table 3.2. Number of Pixels and Ground Sample Distance (GSD) per tile after image preprocessing...................................................... 43

Table 3.3. Comparison of detection quality for images of largest Ground Sample Distance (GSD) per study area for the untiled image and the same image merged from 25 tiles. Lower errors are marked in bold. ......... 46

Table 4.1. Specifications of UAV datasets....................................... 58

Table 4.2. Varying numbers of superpixels $k$ resulting for the two study areas with a coverage of $1,000,000 \mathrm{~m}^{2}$ (Amtsvenn) and $250,000 \mathrm{~m}^{2}$ (Toulouse).

Table 5.1. Specifications of UAV data. ....................................... 71

Table 5.2. Features calculated per SLIC line segment......................... 75

Table 5.3. Classification performance: detection quality for SLIC lines of different cost value c compared to reference data....................... 79

Table 5.4. Interactive outlining performance: general statistics for the manual

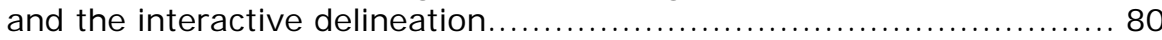

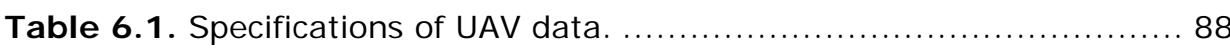

Table 6.2. Features calculated per line to be used by the Random Forest (RF) classifier for boundary classification. ..................................... 93

Table 6.3. Omitted features calculated in previous version of approach [300].

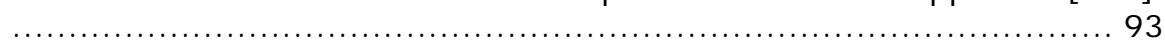

Table 6.4. Specifications of workshops organized for feedback collection. . 99

Table 6.5. Accuracy assessment of building outlines and cadastral boundaries in Rwandan study areas delineated once manually, once with the approach proposed

Table 6.6. Detailed results for building delineation in Rwanda...............101

Table 6.7. Detailed results for cadastral boundary delineation in Rwanda. 101

Table 6.8. Detailed results for cadastral boundary delineation in Kenya...101

Table 6.9. Cases for which the approach proposed fails ordered by frequency, and ideas for improvement. 110

Table 7.1. Distribution of training and testing data for boundary classification based on Random Forest (RF) and Convolutional Neural Networks (CNN).

Table 7.2. Delineation functionalities of BoundaryDelineation QGIS plugin.

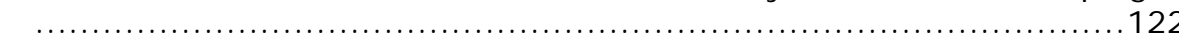

Table 7.3. Settings for our fine-tuned CNN based on VGG19..............127

Table 7.4. Is the boundary likelihood predicted for the correct lines? .......128 
Table 7.5. How correct is the predicted boundary likelihood?...

128

Table 7.6. Does automated delineation cost less effort? .................... 131

Table 7.7. Which plugin functionality to use for which boundary type? ....131

Table 7.8. Results obtained on validation data for different fine-tuned CNNs. 
1. Introduction 


\subsection{Background}

Recording land rights provides land owners tenure security, sustainable livelihood and increases financial opportunities. Estimates suggest that about $75 \%$ of the world population does not have access to a formal system to register and safeguard their land rights. This lack of recorded land rights increases insecure land tenure and fosters existence-threatening conflicts, particularly in developing countries. Recording land rights spatially, i.e., cadastral mapping, is considered the most expensive part of a land administration system. Recent developments in technology allow us to rethink contemporary cadastral mapping. The aim of the its4land project is to make use of technological developments to create more efficient approaches for cadastral mapping.

\subsection{1 its4land Project}

This Ph.D. research is embedded in the its4land project of the European Union (EU) [1]. The project aims to develop an innovative suite of land tenure recording tools inspired by geo-information technologies, that responds to enduser needs and market opportunities in sub Saharan Africa, reinforcing existing strategic collaborations between EU and East Africa [2-4]. The project goals align with target 1.4 of the sustainable development goals (SDGs) of the United Nations, which aims to deliver tenure security for all [5].

The land tenure recording tools are intended to be investigated in terms of integration, validation, demonstration and prototyping in the context of the fitfor-purpose concept for land administration published by the World Bank and the International Federation of Surveyors (FIG) [6]. Fit-for-purpose land administration is part of broader development theories that argue societal prosperity requires secure land tenures provided by a complete and up-to-date land administration system $[7,8]$. its4land investigations are based on case study scenarios in Kenya, Rwanda and Ethiopia. Each country has its specific land tenure recording situation, but all face an immense challenge to rapidly and cheaply map millions of unrecognized land rights. Within this context, the project aims to provide tools for a rapid and cheap mapping of a large amount of unrecognized land rights.

its4land is part of the H2020-ICT-2015 program and has its start and end point on 01 . February 2016 and 31. January 2020, respectively. During those 48 months, eight consortium partners work on research and innovation with the aim of international partnership building in low and middle income counties. The consortium includes multi-sectorial, multi-national and multidisciplinary partners located in Africa and Europe. The group consists of the Technical University of Kenya, the Bahir Dar University, the Institut d'Enseignement Superieur de Ruhengeri and Esri Rwanda, while the latter group consists of the 
University of Twente, the Westfälische Wilhelms-Universität Münster, the Katholieke Universiteit Leuven and Hansa Luftbild AG. The Faculty of GeoInformation Science and Earth Observation (ITC), which is part of the University of Twente is the leading partner of its4land and part of its staff members are supervising this Ph.D. research.

The motivation, objectives and research questions from its4land are used as a basis for this Ph.D. research. The Ph.D. research project makes out one of eight transdisciplinary work packages of its4land. A visual contextualization of all work packages is provided in Figure 1.1. The work package Automate It serves as a basis for this Ph.D. research. It aims at an adaptation of UAV mapping and remote sensing methods for cadastral mapping. Further information on its4land can be found on the project website [9] or via its project number 687828 .



Figure 1.1. Structure of its4land project. This Ph.D. research is based on one of the four technical work packages, namely Automate It.

\subsubsection{Key Concepts}

Unmanned aerial vehicles (UAV), also known as drones, Unmanned Aerial Systems (UAS), or Remotely Piloted Aircraft Systems (RPAS) are small aircraft 
systems without an on-board pilot. They are evolving as an alternative tool to acquire land tenure data. UAVs can capture geospatial data at high quality and resolution in a cost-effective, transparent, and flexible manner, from which visible land parcel boundaries, i.e., cadastral boundaries are extractable. This extraction is not automated, even though physical objects automatically retrievable through image analysis methods often demarcate cadastral boundaries. This Ph.D. research contributes to advancements in developing a corresponding methodology for automated feature extraction from highresolution imagery for cadastral boundary mapping. Automated feature extraction refers to image analysis methods that automatically extract relevant information on visible physical objects. These objects often demarcate cadastral boundaries, which are spatial representations of cadastral records, showing the extent, value, and ownership of land. The process of delineating these boundaries to provide precise spatial description and identification of land parcels is referred to as cadastral boundary mapping. The automated identification and delineation of cadastral boundaries are based on highresolution imagery. These are data of high spatial resolution captured with an optical sensor from a remote sensing platform such as an aircraft or an UAV.

\subsubsection{Application of UAV-based Cadastral Mapping}

In the context of contemporary cadastral mapping, UAVs are increasingly argued and demonstrated as tools able to generate accurate and georeferenced high-resolution imagery from which cadastral boundaries can be visually detected and manually delineated [10-12]. To support this manual delineation, existing parcel boundary lines might be automatically superimposed, which could simplify and accelerate cadastral mapping [13]. Except for [14,15], cadastral mapping is not mentioned in review papers on application fields of UAVs [16-19]. This might be due to the small number of case studies within this field, the often highly prescribed legal regulations relating to cadastral surveys, and the novelty of UAV in mapping generally. Nevertheless, all existing case studies underline the high potential of UAVs for cadastral mapping in both urban and rural contexts for developing and developed countries.

Cadastral mapping contributes to the creation of formal systems for registering and safeguarding land rights. According to the World Bank and the International Federation of Surveyors (FIG), 75\% of the world's population does not have access to such systems. Further, they state that 90 countries lack land registration systems, while 50 countries are in the process of establishing such systems [6]. In these countries, cadastral mapping is often based on partly outdated maps or satellite images of low-resolution, which might include areas covered by clouds. Numerous studies have investigated cadastral mapping based on orthoimages derived from satellite imagery [20- 
27] or aerial photography [28]. The definition of boundary lines is often conducted in a collaborative process among members of the communities, governments and aid organizations, which is referred to as 'Community Mapping' [29], 'Participatory Mapping' [24] or 'Participatory GIS' [20]. Such outdated satellite images are substitutable through up-to-date high-resolution orthoimages derived from UAVs, as shown in case studies in Namibia [10] and Rwanda [12]. The latter case shows the utility of UAVs to partially update existing cadastral maps.

In developed countries, the case studies focus on the conformity of the UAV data's accuracy with local accuracy standards and requirements [30,31]. Furthermore, case studies tend to investigate the possibilities of applying UAVs to reshape the cadastral production line efficiency and effectiveness [32-34]. In the latter, manual boundary detection with all stakeholders is conducted in an office, eliminating the need for convening all stakeholders on the parcel. In developed countries, UAV data are frequently used to update small portions of existing cadastral maps rather than creating new ones. Airspace regulations are the most limiting factor that hinders the thorough use of UAVs. Currently, regulatory bodies face the alignment of economic, information and safety needs or demands connected to UAVs $[18,35]$. Once these limitations are better aligned with societal needs, UAVs might be employed for further fields of land administration, including the monitoring of public infrastructure like oil and gas pipelines, power lines, dikes, highways, and railways [36]. Nowadays, some national mapping agencies in Europe integrate, but mainly investigate, the use of UAVs for cadastral mapping [35].

Overall, UAVs are employed to support land administration both in creating and updating cadastral maps. The entirety of case studies confirms that UAVs are suitable as an addition to conventional data acquisition methods to create detailed cadastral maps, including overview images or 3D models [30,31,37]. The average geometrical precision is shown to be the same, or better, compared to conventional terrestrial surveying methods [32]. UAVs will not substitute conventional approaches since they are currently not suited to map large areas such as entire countries [38]. The use of UAVs supports the economic feasibility of land administration and contributes to the accuracy and completeness of cadastral maps.

\subsubsection{Boundary Delineation for UAV-based Cadastral Mapping}

In all case studies, cadastral boundaries are manually detected and delineated from orthoimages. This is realized either in an office with a small group of involved stakeholders for one parcel or in a community mapping approach for several parcels at once. All case studies lack an automatic approach to extract boundary features from the UAV data. An automatic or semi-automatic feature 
extraction process would simplify cadastral mapping: manual feature extraction is generally regarded as time-consuming, wherefore automation will bring substantial benefits [39]. The degree of automation can range from semiautomatic, including human interaction to fully automatic. Due to the complexity of image understanding, fully automatic feature extraction often shows a certain error rate. Therefore human interaction can hardly be excluded completely [40]. However, even a semi-automatic or partial extraction of boundary features would radically alter cadastral mapping with regards to cost and time. Jazayeri et al. state that UAV data have the potential for automated object reconstruction and boundary extraction activities to be accurate and low-cost [41]. This is especially true for visible boundaries, manifested physically by objects such as hedges, stone walls, large scale monuments, walkways, ditches, or fences, which often coincide with cadastral boundaries $[42,43]$. Such visible boundaries offer the potential to be automatically extracted from UAV data.

\subsection{Research Gap}

UAVs providing high-resolution imagery and automatic feature extraction are novel tools in cadastral boundary mapping. Automated cadastral boundary delineation based on UAV data is rarely investigated, even though physical objects, which can be extracted using image analysis, often demarcate cadastral boundaries. An automated workflow that delineates cadastral boundaries from UAV data offers the potential to improve current cadastral mapping approaches in terms of time, cost, accuracy, and acceptance. At the beginning of this Ph.D. and to the best of our knowledge, no research has been done on expediting the cadastral mapping workflow through automatic boundary delineation from UAV data.

\subsection{Research Objectives}

The main goal of this Ph.D. research is to develop an approach that simplifies image-based cadastral mapping. This aims to support the automated mapping of land tenure. The goal is pursued by developing an automated cadastral boundary delineation approach applicable to high-resolution remote sensing data.

The research addresses the following sub-objectives:

(i) To review relevant information

In the scope of this objective, relevant background information is reviewed. This concerns the state-of-the-art on cadastral mapping, boundary delineation, UAV photogrammetry, and feature extraction. The information is structured to serve as a basis for further developments on automated boundary delineation for UAV-based 
cadastral mapping. To review case studies that deal with UAV-based cadastral mapping aims to demonstrate the potential of UAVs within this field and to outline the lack of an automated approach for boundary delineation.

(ii) To develop a suitable approach

This objective focusses on developing a suitable approach based on the information obtained in (i). While (i) provides contextual information and ideas on how to develop a suitable workflow in theory, this objective focusses on testing and adapting different methods in practice. The objective is pursued by designing and implementing an approach that is applicable to UAV-based cadastral mapping, and that is superior to manual delineation.

(iii) To optimize and evaluate the developed approach

This objective focusses on analyzing the developed approach from (ii) in the context of UAV-based cadastral mapping provided in (i). The developed approach is evaluated in comparison to manual delineation and refined when necessary.

\subsection{Outline}

Objective (i) is addressed in chapters 1 and 3, objective (ii) in chapters 4, 5 and 6 , and objective (iii) in chapters 7 and 8.

The dissertation is structured as follows:

Chapter 1 introduces the Ph.D. research. We describe the research gap to be addressed and formulate corresponding research objectives.

Chapter 2 provides contextual information on the Ph.D. research. This is done by reviewing approaches for feature extraction from various application fields. These are synthesized into a hypothetical workflow applicable for automated boundary delineation from UAV data. The workflow consists of image segmentation, line extraction, and contour generation.

Chapter 3 investigates which method performs best for image segmentation, which is the first step of the hypothetical workflow proposed in chapter 3 . This is done by analyzing the transferability of $\mathrm{gPb}$ contour detection, a state- ofthe-art computer vision method, to UAV-based cadastral mapping.

Chapter 4 investigates which method performs best for line extraction, which is the second step of the hypothetical workflow proposed in chapter 3 . This is done by analyzing a superpixel approach, namely simple linear iterative 
clustering (SLIC), in terms of its applicability to delineate outlines of roads and roofs from UAV data.

Chapter 5 investigates which method performs best for contour generation, which is the third step of the hypothetical workflow proposed in chapter 3 . This is done by coupling gPb contour detection and SLIC superpixels through machine learning with a procedure for a subsequent interactive delineation.

Chapter 6 investigates how to improve the workflow developed. This is done by reducing its complexity: the coupling of $\mathrm{gPb}$ contour detection and SLIC superpixels are replaced by multiscale combinatorial grouping (MCG). The workflow now consists of image segmentation, boundary classification, and interactive delineation. Benefits of the approach compared to manual delineation are analyzed in geometrical, operational, and qualitative regards.

Chapter $\mathbf{7}$ investigates how each step of the workflow can be optimized. For image segmentation, filtering is added to reduce over-segmentation. For boundary classification, Convolution Neural Networks (CNN) replace predicting boundary likelihoods with Random Forest (RF). For interactive delineation, additional functionalities are developed. The effectiveness of the approach compared to manual delineation is evaluated for rural and peri-urban scenes from UAV and aerial data.

Chapter 8 synthesizes the work with conclusions per research objective and reflects upon lessons learned and recommendations for future work. 


\section{Review of Automatic Feature Extraction from High-Resolution Optical Sensor Data for UAV-based Cadastral Mapping*}

* This chapter is based on:

44. Crommelinck, S.; Bennett, R.; Gerke, M.; Nex, F.; Yang, M.Y.; Vosselman, G. Review of Automatic Feature Extraction from High-Resolution Optical Sensor Data for UAV-Based Cadastral Mapping. Remote Sensing 2016, 8, 1-28. 


\begin{abstract}
Unmanned Aerial Vehicles (UAVs) have emerged as a rapid, low-cost and flexible acquisition system that appears feasible for application in cadastral mapping: high-resolution imagery, acquired using UAVs, enables a new approach for defining property boundaries. However, UAV-derived data are arguably not exploited to its full potential: based on UAV data, cadastral boundaries are visually detected and manually delineated. A workflow that automatically extracts boundary features from UAV data could radically increase the pace of current mapping procedures. This review introduces a workflow considered applicable for automated boundary delineation from UAV data. This is done by reviewing approaches for feature extraction from various application fields and synthesizing these into a hypothetical generalized cadastral workflow. The workflow consists of pre-processing, image segmentation, line extraction, contour generation and post-processing. The review lists example methods per workflow step including a description, trialed implementation, and a list of case studies applying individual methods. Furthermore, accuracy assessment methods are outlined. Advantages and drawbacks of each approach are discussed in terms of their applicability on UAV data. This review can serve as a basis for future work on the implementation of most suitable methods in an UAV-based cadastral mapping workflow.
\end{abstract}




\subsection{Introduction}

Unmanned Aerial Vehicles (UAVs) have emerged as rapid, efficient, low-cost and flexible acquisition systems for remote sensing data [14]. The data acquired might be of high-resolution and accuracy, ranging from a sub-meter level to a few centimes $[45,46]$. A photogrammetric UAV workflow includes flight planning, image acquisition, mostly camera calibration, image orientation and data processing, which can result in Digital Terrain Models (DTMs), Digital Surface Models (DSMs), orthoimages and point clouds [39]. UAVs are described as a capable sourcing tool for remote sensing data since they allow flexible maneuverings, high-resolution image capture, flying under clouds, easy launch and landing and fast data acquisition at low cost. Disadvantages include payload limitations, uncertain or restrictive airspace regulations, battery induced short flight duration and time consuming processing of large volumes of data gathered $[47,48]$. In addition, multiple factors that influence the accuracy of derived products require extensive consideration. This includes the quality of the camera, the camera calibration, the number and location of ground control points and the choice of processing software [32]. UAVs have been employed in a variety of applications such as the documentation of archaeological sites and cultural heritage $[49,50]$, vegetation monitoring in favor of precision agriculture [51,52], traffic monitoring [53], disaster management [54,55] and 3D reconstruction [56].

Another emerging application field is UAV-based cadastral mapping. Cadastral maps are spatial representations of cadastre records, showing the extent, value, and ownership of land [57]. Cadastral maps are intended to provide a precise description and identification of land parcels, which are crucial for a continuous and sustainable recording of land rights [7]. Furthermore, cadastral maps support land and property taxation, allow the development and monitoring of a land markets, support urban planning and infrastructure development and allow production of statistical data. An extensive review of concepts and purposes of cadasters in relation to land administration is given in $[58,59]$. UAVs are proposed as a new tool for fast and cheap spatial data production that enable the production of cadastral maps. Within this field, UAVs simplify land administration processes and contribute to securing land tenure [60]. UAVs enable a new approach to the establishment and updating of cadastral maps that contribute to new concepts in land administrations such as fit-for-purpose [6], pro-poor land administration [61] and responsible land administration [24].

\subsubsection{Objective and Organization of the Study}

The review is based on the hypothesis that image processing algorithms applied to high-resolution UAV data are employable to determine cadastral boundaries. Therefore, methods are reviewed that are deemed feasible for 
detecting and extracting cadastral boundaries. The review is intended to serve as a basis for future work on the implementation of the most suitable methods in an UAV-based cadastral mapping workflow. The degree of automation of the final workflow is left undetermined at this point. Due to an absence of work in this context, the scope of this review is extended to methods that could be used for UAV-based cadastral mapping, but that are currently applied (i) on different data sources or (ii) for different purposes.

(i) UAV data includes dense point clouds from which DTMs and DSM are derived as well as high-resolution imagery. Such products can be similarly derived from other high-resolution optical sensors. Therefore, methods based on other highresolution optical sensor data such as High-Resolution Satellite Imagery (HRSI) and aerial imagery are equally considered in this review. Methods applied solely on 3D point clouds are excluded: UAV-derived point clouds do not contain full 3D information since visual information is often lost or generalized. Methods that are based on the derived DSM are considered in this review. Methods that combine 3D point clouds and aerial or satellite imagery are considered in terms of methods based on the aerial or satellite imagery.

(ii) The review includes methods that aim to extract features other than cadastral boundaries having similar characteristics, which are outlined in the next section. Suitable methods are not intended to extract the entirety of boundary features since some boundaries are not visible to optical sensors.

This paper is structured as follows: Firstly, the objects to be automatically extracted are defined and described. Therefore, cadastral boundary concepts and common cadastral boundary characteristics are outlined. Secondly, methods that are feasible to automatically detect and extract previously outlined boundary features are listed. The methods are structured according to subsequently applicable workflow steps. Thereafter, representative methods are applied to an example UAV dataset to visualize their performance and applicability on UAV data. Thirdly, accuracy assessment methods are outlined. Finally, the methods are discussed in terms of the advantages and drawbacks faced in case studies and during the implementation of representative methods. The term 'case studies' is extended to studies on method development followed by examples in this review. The conclusion covers recommendations on suitable approaches for boundary delineation and issues to address in future work. 


\subsection{Review of Feature Extraction and Evaluation Methods}

\subsubsection{Cadastral Boundary Characteristics}

In this paper, a cadastral boundary is defined as a dividing entity with a spatial reference that separates adjacent land plots. An overview on concepts and understandings of boundaries in different disciplines is given in [43]. Cadastral boundaries can be represented in two different ways: (i) in many cases they are represented as line features that clearly demarcate the boundary's spatial position. (ii) Some approaches employ laminar features that represent a cadastral area without clear boundaries. The cadastral boundary is then defined implicitly based on the outline or center of the area constituting the boundary [62]. This is beneficial for ecotones that represent transitional zones between adjacent ecosystems or for pastoralists that move along areas. In such cases, cadastral boundaries seek to handle overlapping access rights and to grant spatiotemporal mobility [63-65]. As shown, a cadastral boundary does not merely include spatial aspects, but those of time and scale as well $[66,67]$.

Different approaches exist to categorize concepts of cadastral boundaries. The lines between the different categories presented in the following can be understood as fuzzy. They are drawn to give a general overview, visualized in Figure 2.1. From a technical point of view, cadastral boundaries are dividable into two categories: (i) Fixed boundaries, whose accurate spatial position has been recorded and agreed upon and (ii) general boundaries, whose precise spatial position is left undetermined [8]. Both require surveying and documentation in cadastral mapping. Cadastral surveying techniques can be distinguished between (i) direct techniques, in which the accurate spatial position of a boundary is measured on the ground using theodolite, total stations and Global Navigation Satellite System (GNSS) and (ii) indirect techniques, in which remotely sensed data such as aerial or satellite imagery are applied. The spatial position of boundaries is derived from these data in a second step [21]. Fixed boundaries are commonly measured with direct techniques, which provide the required higher accuracy. Indirect techniques, including UAVs, are able to determine fixed boundaries only in the case of highresolution data. Indirect techniques are mostly applied to extract visible boundaries. These are determined by physical objects and coincide with the concept of general boundaries $[42,43]$. This review concentrates on methods that delineate general, i.e., visible cadastral boundaries from indirect surveying techniques of high-resolution. The methods are intended to automatically extract boundary features and to be employable to UAV data. 


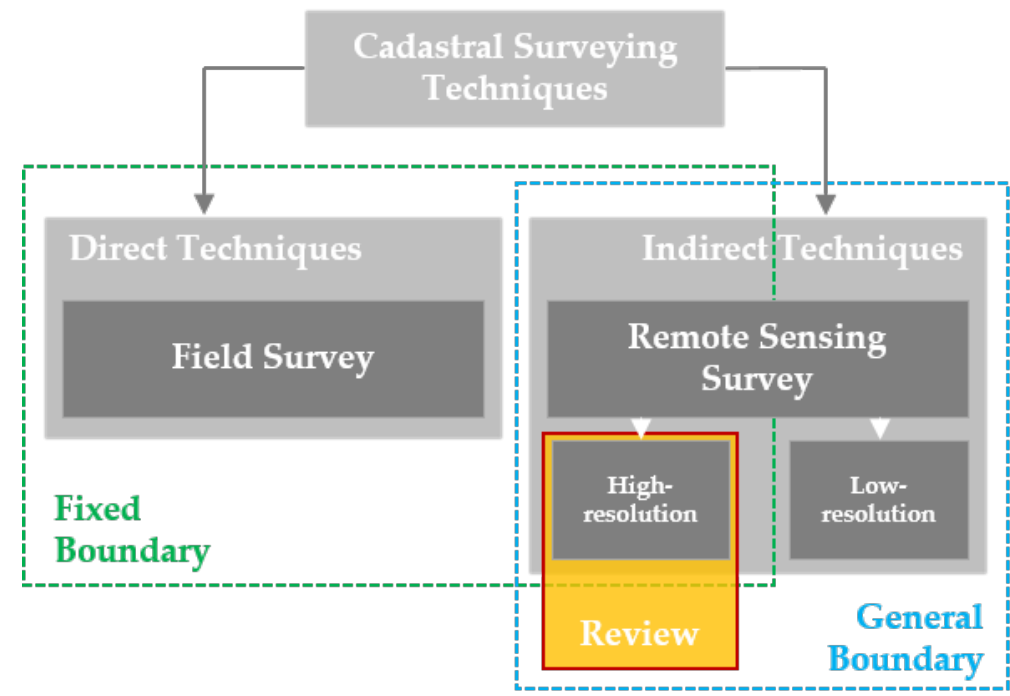

Figure 2.1. Overview of cadastral surveying techniques and cadastral boundary concepts that contextualize the scope of this review paper. The lines between different categories are fuzzy and should not be understood exclusively. They are drawn to give a general overview.

In order to understand, which visible boundaries define the extents of land, literature on 2D cadastral mapping based on indirect techniques was reviewed to identify common boundary characteristics. Man-made objects are found to define cadastral boundaries as well as natural objects. Studies name buildings, hedges, fences, walls, roads, footpaths, pavement, open areas, crop type, shrubs, rivers, canals and water drainages as cadastral boundary features $[10,20,21,23,32,68-70]$. Trees are named as the most limiting factor since they often obscure the view of the actual boundary [31,71]. No study summarizes characteristics of detected cadastral boundaries, even though it is described as crucial for feature recognition to establish a model that describes the general characteristics of the feature of interest [72]. Common in many approaches is the linearity of extracted features. This might be due to the fact that some countries do not accept curved cadastral boundaries [22]. Even if a curved river marks the cadastral boundary, the boundary line is approximated by a polygon [21]. When considering the named features, the following characteristics can be derived: most features have a continuous and regular geometry expressed in long straight lines of a limited curvature. Furthermore, features often share common spectral properties, such as similar values in color and texture. Moreover, boundary features are topologically connected and form a network of lines that surround land parcels of a certain (minimal) size and shape. Finally, boundaries might be indicated by a special distribution of other objects such as trees. In summary, features are detectable based on their geometry, spectral property, topology and context. 
This review focusses on methods that extract linear boundary features since cadastral boundaries are commonly represented by straight lines with exceptions outlined in $[64,73]$. Cadastral representations in 3D as described in [74] are excluded. With UAVs, not all cadastral boundaries can be detectable. Only those detectable with an optical sensor, i.e., visible boundaries can be extracted. This approach does not consider socially perceived boundaries that are not marked by a physical object. Figure $\mathbf{2 . 2}$ provides an overview of visible boundary characteristics mentioned before and commonly raised issues in terms of their detection.

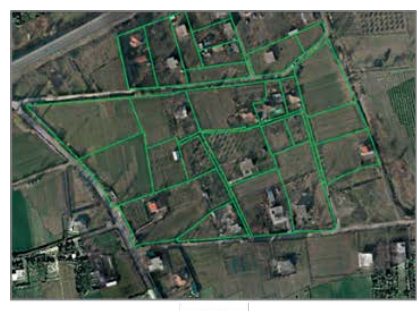

(a)

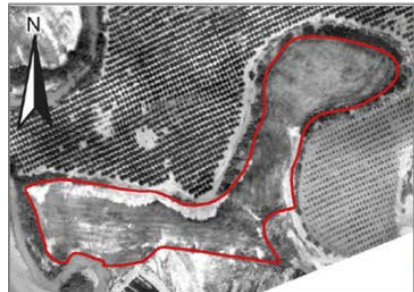

(d)

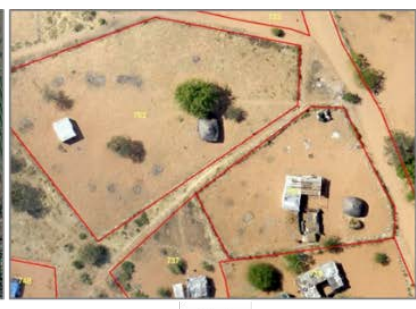

(b)

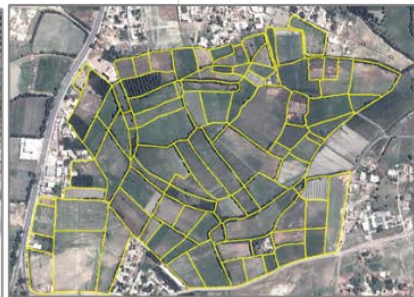

(e)

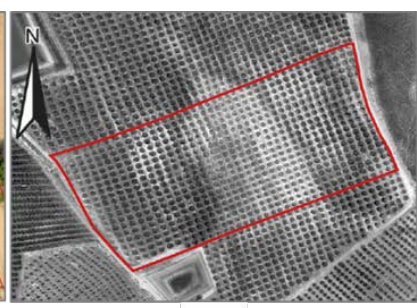

(c)

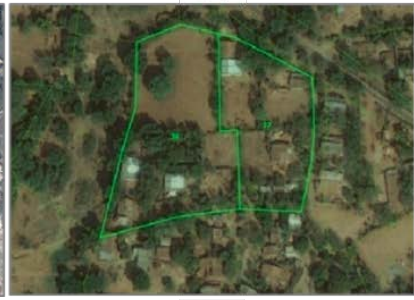

(f)

Figure 2.2. Characteristics of cadastral boundaries extracted from high-resolution optical remote sensors. The cadastral boundaries are derived based on (a) roads, power lines and pipelines [38], (b) fences and hedges [10], (c/ d) crop types [31], (e) roads, foot paths, water drainage, open areas and scrubs [75], and (f) adjacent vegetation [71]. (d) Shows the case of a nonlinear irregular boundary shape. The cadastral boundaries in (e) and (f) are often obscured by tree canopy. Cadastral boundaries in (ad) are derived from UAV data; in (e) and (f) from HRSI. All of the boundaries are manually extracted and delineated.

\subsubsection{Feature Extraction Methods}

This section reviews methods that are able to detect and extract the above mentioned boundary characteristics. The methods reviewed are either pixelbased or object-based. (i) Pixel-based approaches analyze single pixels, optionally taking into account the pixels' context, which can be considered through moving windows or implicitly through modeling. These data-driven approaches are often employed when the object of interest is smaller or similar in size as the spatial resolution. Example exceptions are modern convolutional neural networks (CNN) [76], which are explained in the latter. The lack of an explicit object topology is one drawback that might lead to inferior results, in 
particular for topographic mapping applications compared to those of human vision [77]. (ii) Object-based approaches are employed to explicitly integrate knowledge on object appearance and topology into the object extraction process. Applying these approaches becomes possible, once the spatial resolution is finer than the object of interest. In such cases, pixels with similar characteristics such as color, tone, texture, shape, context, shadow or semantics are grouped to objects. Such approaches are referred to as Object Based Image Analysis (OBIA). They are considered model-driven since knowledge about scene understanding is incorporated to structure the image content spatially and semantically. The grouping of pixels might also results into groups of pixels, called superpixels. This approach with corresponding methods could be seen as a third in-between category, but is understood as object-based in this review [78-80].

Pixel-based approaches are often used to extract low-level features, which do not consider information about spatial relationships. Low-level features are extracted directly from the raw, possibly noisy pixels with edge detection being the most prominent algorithms [81]. Object-based approaches are used to extract high-level features, which represent shapes in images that are detected invariant of illumination, translation, orientation and scale. High-level features are mostly extracted based on the information provided by low-level features [81]. High-level feature extraction aimed at automated object detection and extraction, is currently achieved in a stepwise manner and is still an active research field [82]. Algorithms for high-level feature extraction often need to be interlinked to a processing workflow and do not lead to appropriate results when applied solely [78]. The relation of the described concepts is visualized in Figure 2.3. Both pixel-based and object-based approaches are applicable to UAV data. Pixel-based approaches can be applied to UAV data, or to its down sampled version of lower resolution. Due to the high-resolution of UAV data, object-based approaches seem to be preferred. The final boundary representation should be object-based rather than pixel-based. Both approaches are included in this review as the ability to discriminate and extract features is highly dependent on scale $[83,84]$. 


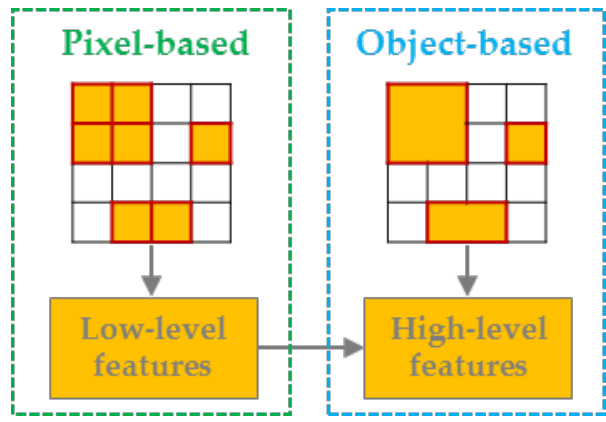

Figure 2.3. Pixel-based and object-based feature extraction approaches aim to derive low-level and high-level features from images. Object-based approaches may include information provided by low-level features that is used for high-level feature extraction.

The reviewed methods are structured according to a sequence of commonly applied workflow steps for boundary delineation, shown in Figure 2.4. The structure of first identifying candidate regions, then detecting linear features, and finally connecting these appears to be a generic approach, as following literature exemplifies: A review of linear feature extraction from imagery [72], a review of road detection [85] and case studies that aim to extract road networks from aerial imagery $[86,87]$ and to delineate tree outlines from HRSI [88]. The first step, image segmentation, aims to divide an image into nonoverlapping segments in order to identify candidate regions for further processing [89-91]. The second step, line extraction, detects edges. Edges are defined as a step change in the value of a low-level feature such as brightness or color. A collinear collection of such edges aggregated on the basis of a grouping criteria is commonly defined as a line [92-94]. The third step, contour generation, connects lines to form a closed vectorized boundary line that surrounds an area defined through segmentation. These main steps can optionally be extended with pre- and post-processing steps.

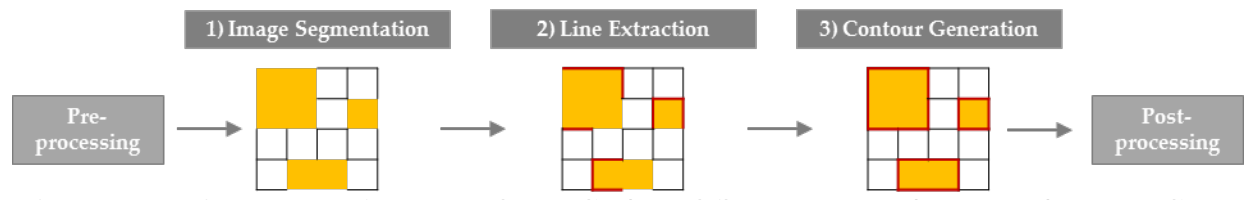

Figure 2.4. Sequence of commonly applied workflow steps to detect and extract linear features used to structure the methods reviewed.

This review includes 37 case studies of unknown resolution and 52 case studies of multiple resolutions, most often below $5 \mathrm{~m}$ (Figure 2.5). The investigated case studies intend to detect features such as coastlines, agricultural field boundaries, road networks and buildings from aerial or satellite imagery, which is mainly collected with IKONOS or QuickBird satellites. The methods are often equally applicable to aerial and satellite imagery, as the data sources can have similar characteristics such as the high-resolution of the derived orthoimages [95]. 




Figure 2.5. Spatial resolution of data used in the case studies. The figure shows the 52 case studies, in which the spatial resolution was known. For case studies that use datasets of multiple resolutions, the median resolution is used. For 37 further case studies, which are not represented in the histogram, the spatial resolution was left undetermined.

In the following, each workflow step is explained in detail, including a table of example methods and case studies that apply these methods. The table represents possible approaches, with various further methods possible. The most common strategies are covered, while specific adaptations derived from these are excluded, to limit the extent of this survey. Overall, the survey of methods in this review is extensive, but it does not claim to be complete. The description and contextualization of most methods is based upon [96-99]. Due to the small number of case studies on linear feature extraction that employ high-resolution sensors of $<0.5 \mathrm{~m}$, one group of the described table includes case studies on resolutions of up to $5 \mathrm{~m}$, whereas the other includes the remaining case studies. In order to demonstrate the applicability of the methods on UAV imagery for boundary delineation, some representative methods were implemented. An orthoimage acquired with a fixed-wing UAV during a flight campaign in Namibia served as an exemplary dataset (Figure 2.6). The orthoimage shows a rural residential housing area and has a Ground Sample Distance (GSD) of $5 \mathrm{~cm}$. The acquisition and processing of the images is described in [10]. Cadastral boundaries are marked with fences and run along paths in this exemplary dataset. As for the implementation, image processing libraries written in Python and Matlab were considered. For Python, this included Scikit [100] and OpenCV modules [101]. The latter are equally available in $\mathrm{C}++$. For Matlab, example code provided from MathWorks [102] and VLFeat [103] was adopted. The methods were implemented making use of different libraries and mostly applying standard parameters. The visually most representative output was chosen for this review as an illustrative explanation of discussed methods. 




Figure 2.6. UAV-derived orthoimage that shows a rural residential housing area in Namibia, which is used as an exemplary dataset to implement representative feature extraction methods.

\subsubsection{Preprocessing}

Pre-processing steps might be applied in order to improve the output of the subsequent image segmentation and to simplify the extraction of linear features. Therefore, the image is processed to suppress noise and enhance image details. The pre-processing includes the adjustment of contrast and brightness and the application of smoothing filters to remove noise [104]. Two possible approaches that aim at noise removal and image enhancement are presented in the following. Further approaches can be found in [105].

- Anisotropic diffusion aims at reducing image noise while preserving significant parts of the image content (Figure 2.7), based on source code provided in [106]). This is done in an iterative process of applying an image filter until a sufficient degree of smoothing is obtained [106,107].

- Wallis filter is an image filter method for detail enhancement through local contrast adjustment. The algorithm subdivides an image into non-overlapping windows of the same size to then adjust the contrast and minimize radiometric changes of each window [108].

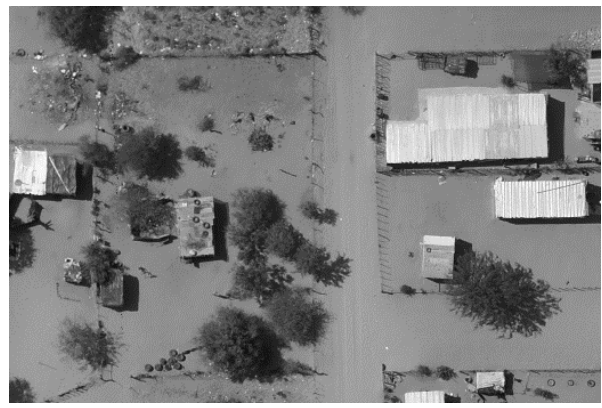

(a)

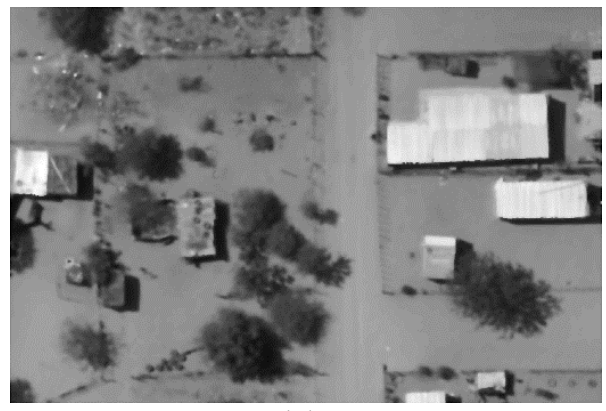

(b)

Figure 2.7. (a) Subset of the original UAV orthoimage converted to greyscale. (b) Anisotropic diffusion applied to greyscale UAV image to reduce noise. After filtering, the image appears smoothed with sharp contours removed, which can be observed at the rooftops and tree contours. 


\subsubsection{Image Segmentation}

This section describes methods that divide an image into non-overlapping segments that represent areas. The segments are detected based on homogeneity parameters or on the differentiation to neighboring regions [109]. In a not ideal case, the image segmentation creates segments that cover more than one object of interest or the object of interest is subdivided into several objects. These outcomes are referred to as undersegmentation and oversegmentation, respectively [109]. Various strategies exist to classify image segmentation, as shown in $[110,111]$. In this review, the methods are classified into (i) unsupervised or (ii) supervised approaches. Table $\mathbf{2 . 1}$ shows an exemplary selection of case studies that apply the methods described in the following.

(i) Unsupervised approaches include methods in which segmentation parameters are defined that describe color, texture, spectral homogeneity, size, shape, compactness and scale of image segments. The challenge lies within defining appropriate segmentation parameters for features varying in size, shape, scale and spatial location. Thereafter, the image is automatically segmented according to these parameters [98]. Popular approaches are described in the following and visualized in Figure 2.8: these were often applied in the case studies investigated for this review. A list of further approaches can be found in [110].

- Graph-based image segmentation is based on color and is able to preserve details in low-variability image regions while ignoring details in high-variability regions. The algorithm performs an agglomerative clustering of pixels as nodes on a graph such that each superpixel is the minimum spanning tree of the constituent pixels [112,113].

- Simple Linear I terative Clustering (SLIC) is an algorithm that adapts a k-mean clustering approach to generate groups of pixels, called superpixels. The number of superpixels and their compactness can be adapted within the memory efficient algorithm [114].

- Watershed algorithm is an edge-based image segmentation method. It is also referred to as a contour filling method and applies a mathematical morphological approach. First, the algorithm transforms an image into a gradient image. The image is seen as a topographical surface, where grey values are deemed as elevation of the surface of each pixel's location. Then, a flooding process starts in which water effuses out of the minimum grey values. When the flooding across two minimum values converges, a boundary that separates the two identified segments is defined $[109,110]$.

- Wavelet transform analyses textures and patterns to detect local intensity variations and can be considered as a generalized combination of three other operations: Multi-resolution analysis, template matching and frequency domain analysis. The algorithm decomposes an image into a low 
frequency approximation image and a set of high frequency, spatially oriented detailed images [115].

- Globalized Probability of Boundary ( $\mathbf{g} \mathbf{P b}$ ) originates from computer vision and proposes closed boundaries by combining edge detection and hierarchical image segmentation, while integrating image information on texture, color and brightness on both a local and a global scale [116].

- Multiscale Combinatorial Grouping (MCG) develops gPb further by a combinatorial merging of the hierarchical regions detected before based on normalized-cut segmentation [117].

(ii) Supervised methods often consist of methods from machine learning and pattern recognition. These can be performed by learning a classifier to capture the variation in object appearances and views from a training dataset. In the training dataset, object shape descriptors are defined and used to label the training dataset. Then, the classifier is learned based on a set of regions with object shape descriptors resulting in their corresponding predicted labels. The automation of machine learning approaches is limited since the classifier needs to be trained with samples that often require manual labeling. The aim of training is to model the process of data generation such that it can predict the output for unforeseen data. Various possibilities exist to select training sets and features [118] as well as to select a classifier [98,119]. In contrast to the unsupervised methods, these methods go beyond image segmentation as they additionally add a semantic meaning to each segment. A selection of popular approaches that have been applied in case studies investigated for this review are described in the following. A list of further approaches can be found in [98].

- Convolutional Neural Networks (CNN) are inspired by biological processes being made up of neurons that have learnable weights and biases. The algorithm creates multiple layers of small neuron collections which process parts of an image, referred to as receptive fields. Then, local connections and tied weights are analyzed to aggregate information from each receptive field [104].

- Markov Random Fields (MRF) are a probabilistic approach based on graphical models. They are used to extract features based on spatial texture by classifying an image into several regions or classes. The image is modelled as a MRF and a maximum a posteriori probability approach is used for classification [120].

- Support Vector Machines (SVM) consist of a supervised learning model with associated learning algorithms that support linear image classification into two or more categories through data modelling. Their advantages include excellent generalization capability, which concerns the ability to classify shapes that are not within the feature space used for training [121]. 
Table 2.1. Case study examples for image segmentation methods.

\begin{tabular}{|c|c|c|c|}
\hline $\begin{array}{r}\text { I mage } \\
\text { Segmentation } \\
\text { Method }\end{array}$ & $\begin{array}{l}\text { Resolution } \\
\quad<5 \mathrm{~m}\end{array}$ & $\begin{array}{l}\text { Resolution } \\
>5 \mathrm{~m}\end{array}$ & $\begin{array}{l}\text { Unknown } \\
\text { Resolution }\end{array}$ \\
\hline Unsupervised & $\begin{array}{l}{[122][123][124]} \\
{[125][126][127]} \\
{[128][129][130]} \\
{[131][132][133]} \\
{[134][135][136]} \\
{[137][87][88]} \\
{[138][116][117]}\end{array}$ & $\begin{array}{l}{[139][140][141]} \\
{[115][142][143]}\end{array}$ & $\begin{array}{l}{[144][145][146]} \\
{[147][148][149]} \\
{[145][135][150]} \\
{[151][152][153]} \\
{[154][155]}\end{array}$ \\
\hline Supervised & $\begin{array}{l}{[83][156]} \\
{[80][158]} \\
{[160][159]} \\
{[161]} \\
{[163][118]} \\
{[165]}\end{array}$ & [166] [167] & $\begin{array}{lll}{[148]} & {[94]} & {[147]} \\
{[168]} & & {[169]} \\
{[170]} & & \end{array}$ \\
\hline
\end{tabular}

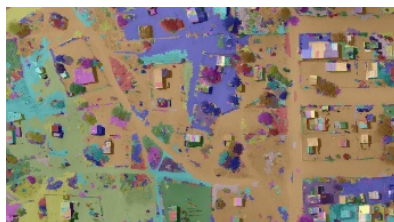

(a)

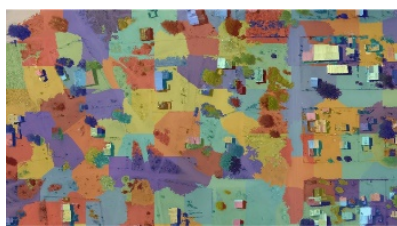

(b)

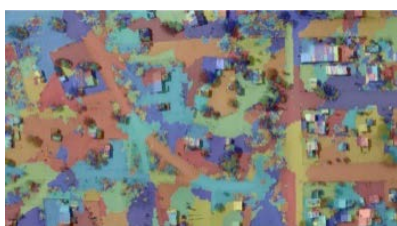

(c)

Figure 2.8. I mage segmentation applied to the original UAV orthoimage: (a) graphbased segmentation, (b) SLIC segmentation, and (c) Watershed segmentation. The labe matrices are converted to colors for visualization purpose. The input parameters are tuned to obtain a comparable number of segments from each segmentation approach. However, all approaches result in differently located and shaped segments.

\subsubsection{Line Extraction}

This section describes methods that detect and extract linear features. Table 2.2 shows an exemplary selection of case studies that apply the described methods, which are visualized in Figure 2.9. The figure shows that a large number of edges is detected especially in the case of vegetation and on the rooftops of buildings, while a small number of edges is detected on paths.

- Edge detection can be divided into (i) first and (ii) second order derivative based edge detection. An edge has the one-dimensional shape of a ramp and calculating the derivative of the image can highlight its location. (i) First order derivative based methods detect edges by looking for the maximum and minimum in the first derivative of the image to locate the presence of the highest rate of change between adjacent pixels. The most prominent representative is the Canny edge detection that fulfils the criteria of a good detection and localization quality and the avoidance of multiple responses. These criteria are combined into one optimization criteria and solved using the calculus of variations. The algorithm consists of Gaussian smoothing, gradient filtering, non-maximum suppression and 
hysteresis thresholding [171]. Further representatives based on first order derivatives are the Robert's cross, Sobel, Kirsch and Prewitt operators. (ii) Second order derivative based methods detect edges by searching for zero crossings in the second derivative of the image to find edges. The most prominent representative is the Laplacian of Gaussian, which highlights regions of rapid intensity change. The algorithm applies a Gaussian smoothing filter, followed by a derivative operation [172,173].

- Straight line extraction is mostly done with the Hough transform. This is a connected component analysis for line, circle and ellipse detection in a parameter space, referred to as Hough space. Each candidate object point is transformed into Hough space, in order to detect clusters within that space that represent the object to be detected. The standard Hough transform detects analytic curves, while a generalized Hough transform can be used to detect arbitrary shaped templates [174]. As an alternative, the Line Segment Detector (LSD) algorithm could be applied. For this method, the gradient orientation that represents the local direction of the intensity value, and the global context of the intensity variations are utilized to group pixels into line-support regions and to determine the location and properties of edges [92]. The method is applied for line extraction in $[\mathbf{1 7 5}, 176]$. The visualization in Figure $\mathbf{2 . 9}$ is based on source code provided in [176].

Table 2.2. Case study examples for line extraction methods.

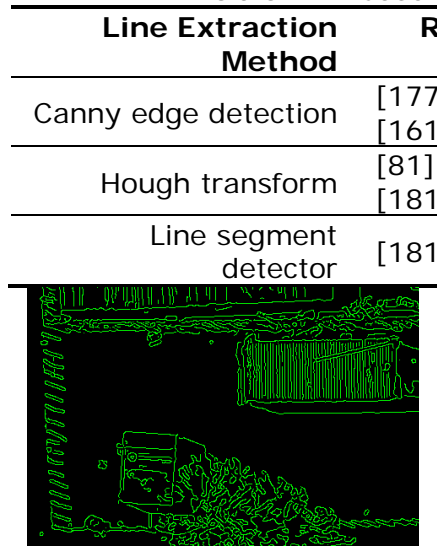

(a)

\section{Resolution} $<5$ m

177] [83] [131

161]

81] [130] [136

81] [175] [138]

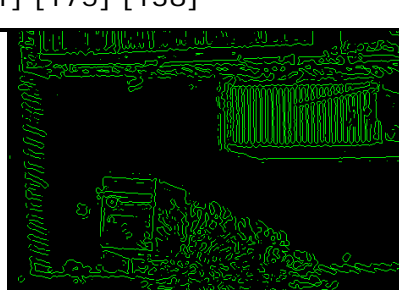

(b) Resolution $>5 \mathrm{~m}$ [139] [178]

[182]

2]

Figure 2.9. Edge detection applied to the greyscale UAV orthoimage based on (a) Canny edge detection and (b) the Laplacian of Gaussian. The output is a binary image in which one value represents edges (green) and the other value represents the background (black). (c) Shows the line segment detector applied and imposed on the original UAV orthoimage.

\subsubsection{Contour Generation}

This section describes methods that are used to generate a vectorized and topologically connected network through connection of line segments. 
Table 2.3 shows an exemplary selection of case studies that apply the methods described in the following, which can be categorized in two groups:

(i) A human operator outlines a small segment of the feature to be extracted. Then, a line tracking algorithm recursively predicts feature characteristics, measures these with profile matching and updates the feature outline respectively. The process continues until the profile matching fails. Perceptual grouping, explained in the following, can be used to group feature characteristics. Case studies that apply such line tracking algorithms can be found in $[184,188,189]$.

(ii) Instead of outlining a small segment of the feature to be extracted, the human operator can also provide a rough outline of the entire feature. Then, an algorithm applies a deformable template and refines this initial template to fit the contour of the feature to be extracted. Snakes, which are explained in the following, are an example for this procedure.

- Perceptual grouping is the ability to impose structural organization on spatial data based on a set of principles namely proximity, similarity, closure, continuation, symmetry, common regions and connectedness. If elements are close together, similar to one another, form a closed contour, or move in the same direction, then they tend to be grouped perceptually. This allows to group fragmented line segments to generate an optimized continuous contour [190]. Perceptual grouping is applied under various names such as line grouping, linking, merging or connection in the case studies listed in Table 2.3.

- Snakes also referred to as active contours are defined as elastic curves that dynamically adapt a vector contour to a region of interest by applying energy minimization techniques that express geometric and photometric constraints. The active contour is a set of points that aims to continuously enclose the feature to be extracted [191]. They are listed here, even though they could also be applied in previous steps, such as image segmentation [122,127]. In this step, they are applied to refine the geometrical outline of extracted features $[88,141,145]$.

Table 2.3. Case study examples for contour generation methods.

\begin{tabular}{|c|c|c|c|}
\hline $\begin{array}{r}\text { Contour Generation } \\
\text { Method }\end{array}$ & $\begin{array}{l}\text { Resolution } \\
<5 \mathrm{~m}\end{array}$ & $\begin{array}{l}\text { Resolution } \\
>5 \mathrm{~m}\end{array}$ & $\begin{array}{c}\text { Unknown } \\
\text { Resolution }\end{array}$ \\
\hline Perceptual grouping & $\begin{array}{l}{[125][158][192]} \\
{[123][138]}\end{array}$ & [178] & $\begin{array}{l}{[152][193][194]} \\
{[195][196][197]} \\
{[198][167][151]} \\
{[154][155][170]} \\
{[187]}\end{array}$ \\
\hline Snakes & $\begin{array}{l}{[122][127][199]} \\
{[200][201][202]} \\
{[88]}\end{array}$ & & [188] [145] [141] \\
\hline
\end{tabular}




\subsubsection{Postprocessing}

Postprocessing aims to improve the output of the delineated feature by optimizing its shape. Two prominent approaches are explained in the following. Table 2.4 shows an exemplary selection of case studies that apply the described post-processing methods, which are visualized in Figure 2.10.

- Douglas-Peucker algorithm is used to simplify a line by reducing the number of points in a curve that is approximated by a series of points [203].

- Morphological operators are employed as a post-processing step to smooth the contour of detected line features [204].

Table 2.4. Case study examples for post-processing methods.

\begin{tabular}{|c|c|c|c|}
\hline $\begin{array}{r}\text { Postprocessing } \\
\text { Method }\end{array}$ & $\begin{array}{c}\text { Resolution } \\
<5 \mathrm{~m}\end{array}$ & $\begin{array}{c}\text { Resolution } \\
>5 \mathrm{~m}\end{array}$ & $\begin{array}{c}\text { Unknown } \\
\text { Resolution }\end{array}$ \\
\hline $\begin{array}{r}\text { Douglas-Peucker } \\
\text { algorithm }\end{array}$ & $\begin{array}{lll}{[80]} & {[192]} & {[87]} \\
{[205][181]} & \\
{[1}\end{array}$ & [178] [143] & [206] \\
\hline $\begin{array}{r}\text { Morphological } \\
\text { operators }\end{array}$ & $\begin{array}{l}{[81][125][126]} \\
{[207][130][83]} \\
{[156][131][208]} \\
{[158][136][87]} \\
{[205][138]}\end{array}$ & [142] & $\begin{array}{l}{[134][135][152]} \\
{[209][154]}\end{array}$ \\
\hline
\end{tabular}






(a)

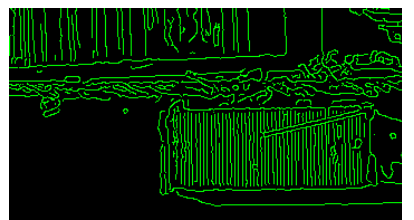

(b)



(c)

Figure 2.10. (a) Douglas-Peucker simplification (red) of the contour generated with snakes (green). The simplified contour approximates the fence that marks the cadastral boundary better than the snake contour does. (b) Binary image derived from Canny edge detection as shown in Figure 2.9a. The image serves as a basis for morphological closing, shown in (c). Through dilation followed by erosion, edge pixels (green) belonging to one class in (b) are connected to larger regions in (c).

\subsubsection{Accuracy Assessment Methods}

In the following, approaches that assess the accuracy of extracted linear features are described. In order to quantify the accuracy, reference data are required to then calculate a metric, which measures the similarity between the result and the reference data. These methods are known as supervised discrepancy methods [210]. The reference data can be acquired through manual delineation of visually extractable linear features $[72,211,212]$ or through their extraction from existing maps [156,208,213]. Some authors extend the assessment to aspects such as time, cost and energy savings and include further accuracy measures [72]. For methods intending to classify linear features, the accuracy assessment is extended to thematic aspects $[214,215]$. In such cases, the confusion matrix is calculated as well as statistics derived from it, such as the user's, producer's and overall accuracy as well as the kappa coefficient $[123,216-218]$. The accuracy might also be evaluated based on thematic and geometric aspects [219]. The geometric accuracy incorporates positional aspects, indicating errors in terms of the object's location and errors in terms of the spatial extent of an object. These components can be assessed with pixel-based and object-based measures. Pixel-based accuracy assessment has a rather quantitative character, is often used to assess geometric accuracy and is more standardized than object-based accuracy assessment. The latter has a rather qualitative character and is often used to assess classification quality [153]. The trend towards standardized pixel-based accuracy measures is manifested in efforts from the International Society for Photogrammetry and Remote Sensing (ISPRS), which publishes benchmark data to assess different methods in a uniform approach [220]. A comparison of both approaches shows that object-based approaches provide additional accuracy information compared to pixel-based approaches [216]. One example for this additional information are topological aspects that can be assessed with an object-based approach as shown in [221]. Such approaches can be based on a fuzzy representation of the object's boundary [222,223]. Ultimately, different aspects in terms of feature extraction performance can be highlighted with a combination of pixel-based and object-based metrics [224]. 
The following approaches that can be applied both pixel-based and objectbased, calculate planimetric accuracy. They are simple to implement and are often applied when assessing feature extraction methods [ $212,216,224,225]$ :

- The completeness measures the percentage of the reference data which is explained by the extracted data, i.e., the percentage of the reference data which could be extracted. The value ranges from 0 to 1 , with 1 being the optimum value.

- The correctness represents the percentage of correctly extracted data, i.e., the percentage of the extraction, which is in accordance with the reference data. The value ranges from 0 to 1 , with 1 being the optimum value.

- The redundancy represents the percentage to which the correct extraction is redundant, i.e., extracted features overlap themselves. The value ranges from 0 to 1 , with 0 being the optimum value.

- The Root-Mean Square (RMS) difference expresses the average distance between the matched extracted and the matched reference data, i.e., the geometrical accuracy potential of the extracted data. The optimum value for RMS is 0 .

These planimetric measures calculate per pixel or per object the number of (i) True Positives (TP), where the extracted features match the reference data, (ii) False Positives (FP), where the extracted feature does not exist in the reference data and (iii) False Negatives (FN), where the features existent in the reference data are not extracted. The measures described can be used to derive further quality measures. These include the quality, the rank distance, the branching factor, the mean detour factor and connectivity measures when assessing linear networks $[181,212,226,227]$. Further, the precision-recall curve, the F-measure and the average precision are derived to assess object detection methods [98]. Since they contain the information inherent in the initial measures, they are used if a single quality measure is desired.

In the most common and simple approach, a buffer area of a specific width is calculated around linear features in the extracted data and the reference data. Comparing these areas then leads to the described accuracy measures. A buffer analysis can be performed either on a pixel-based or object-based representation of linear features. For a pixel-based representation, the buffer consists of a set of pixels within a specific distance from a set of pixels that represents the line. For an object-based representation, i.e., a vector representation, the buffer consists of a corridor of a specific width around the line [228]. The results of this approach strongly depend on the buffer width. 


\subsection{Discussion}

In the following sections, the previously described feature extraction and evaluation methods are discussed and interpreted in perspective of previous studies and in the context of UAV-based cadastral mapping. The former is based on advantages and drawbacks faced in case studies, while the latter is based on the experiences made during the implementation of representative methods. The discussion is structured equivalent to the previous chapter.

\subsubsection{Cadastral Boundary Characteristics}

For all feature extraction approaches it is necessary to define characteristics of the feature to be extracted as stressed in $[72,229]$. Defining feature characteristics has been done in this review for boundary features in terms of geometry, spectral property, topology and context. This is often not exclusively done in the reviewed case studies, which extract linear features such as coastlines, agricultural field boundaries, road networks and buildings. One example of a feature description approach similar to this review is employed in a study on the extraction of road networks [189], in which the feature description is extended to functional characteristics. Even with a thorough description of linear boundary features, methods that extract visible features only are not capable of extracting the entirety of boundary features. This might include socially constructed boundaries that are not visible to an optical sensor $[42,66]$. The detection of such boundaries can be supported through modeldriven approaches that generate a contour around land plots based on a priori knowledge.

\subsubsection{Feature Extraction Methods}

The methods reviewed in this study are grouped according to common workflow steps applied for linear feature extraction from high-resolution optical sensor data. They consist of (i) preprocessing, (ii) image segmentation, (iii) line extraction, (iv) contour generation and (v) post-processing (Figure 2.4). An entirety of methods has been reviewed, which does not result in a recommendation of one specific method per workflow step since further experimental work would be required to do this reliably. The most common methods listed in this review are applied to data of different resolutions mostly below $5 \mathrm{~m}$. Furthermore, the workflow steps are not necessarily independent and sometimes the same method can be applied within different steps. Finding a non-redundant classification structure for all methods does not seem feasible as stated by authors of similar review papers on road extraction [230], object detection [98] and linear feature extraction [72]. Many case studies combine strategies from a variety of approaches, of which many example combinations are listed in [230]. The greatest variety can be found in case studies on road detection [231]. This application field appears to be most prominent for linear 
feature extraction, demonstrated in the large number of case studies, their comprehensive review in [230], their extensive comparison on benchmark data [232] and commonly applied accuracy assessment methods originating from this field [225].

In the following sections, advantages and drawbacks named in case studies that apply previously described methods are outlined. Furthermore, recommendations on their applicability for UAV-based boundary delineation are drawn, when possible.

(i) Preprocessing steps that include image enhancement and filtering are often applied in case studies that use high-resolution data below $1 \mathrm{~m}[128,131,136]$. This might be due to the large level of detail in such images, which can be reduced with filtering techniques. Without such preprocessing, oversegmentation might result - as well as too many non-relevant edges obtained through edge detection. One drawback of applying such preprocessing steps is the need to set thresholds for image enhancement and filtering. Standard parameters might lead to valuable results, but might also erase crucial image details. Selecting parameters hinders the automation of the entire workflow.

(ii) Image segmentation is listed as a crucial first step for linear feature extraction in corresponding review papers $[72,85,119]$. Overall, image segments distinct in color, texture, spectral homogeneity, size, shape, compactness and scale are generally better distinguishable than images that are inhomogeneous in terms of these aspects. The methods reviewed in this paper are classified into supervised and unsupervised approaches. More studies apply an unsupervised approach, which might be due to their higher degree of automation. The supervised approaches taken from machine learning suffer from their extensive input requirements, such as the definition of features with corresponding object descriptors, labeling of objects, training a classifier and applying the trained classifier on test data [94,157]. Furthermore, the ability of machine learning approaches to classify an image into categories of different labels is not necessarily required in the scope of this workflow step since the image only needs to be segmented. The proposed machine learning approaches, such as CNNs, can also be employed in further workflow steps, i.e., for edge detection as shown in [233]. A combination of edge detection and image segmentation based on machine learning is proposed in [234]. According to Dey et al. it is challenging for CNNs to decide on a general network structure and to properly learn the classifier with test data [110]. A large number of case studies are based on SVM [121]. SVMs are appealing due to their ability to generalize well from a limited amount and quality of training data, which appears to be a common limitation in remote sensing. Mountrakis et al. found that SVMs can be based on fewer training 
data, compared to other approaches. However, they state that selecting parameters such as kernel size strongly affects the results and is frequently solved in a trial-and-error approach, which again limits the automation [121]. Furthermore, SVMs are not optimized for noise removal, which makes image preprocessing indispensable for high-resolution data. Approaches such as the Bag-of-Words framework, as applied in [235], have the advantage of automating the feature selection and labeling, before applying a supervised learning algorithm. Further state-of-the-art approaches, including AdaBoost and random forest are discussed in [98]. Machine learning approaches can also consist of segmentation and

(iii) Line extraction makes up the majority of case studies on linear feature extraction, with Canny edge detection being the most prominent approach. The Canny edge detector is capable of reducing noise while a second order derivative such as the Laplacian of Gaussian that responds to transitions in intensity, is sensitive to noise. When comparing different edge detection approaches, it has been shown that the Canny edge detector performs better than the Laplacian of Gaussian and first order derivatives as the Robert's cross, Sobel and Prewitt operator [172,173]. In terms of line extraction, the Hough transform is the most commonly used method. The LSD appears as an alternative that requires no parameter tuning while giving accurate results.

(iv) Contour Generation is not represented in all case studies since it is not as essential as the two previous workflow steps for linear feature extraction. The exceptions are case studies on road network extraction, which name contour generation, especially based on snakes, as a crucial workflow step $[85,230]$. One drawback of snakes lies in finding a seed point as the snake should be initialized close to features to be extracted [202]. Furthermore, these methods require parameter tuning in terms of the energy field, which limits their automation [88]. Perceptual grouping is rarely applied in the case studies investigated, especially not in those based on high-resolution data.

(v) Postprocessing is utilized more often than preprocessing. Especially morphological filtering is applied in the majority of case studies. Admittedly, it is not always employed as a form of post-processing to improve the final output, but equally during the workflow to smooth the result of a workflow step before further processing $[81,130,131,136]$. When applied at the end of the workflow in case studies on road extraction, morphological filtering is often combined with skeletons to extract the vectorized centerline of the road $[87,135,152,205,207]$. 


\subsubsection{Accuracy Assessment Methods}

Considering the accuracy, there is not one optimal method for its assessment or reporting. Many studies, especially those that aim at road extraction, use the described approach based on buffer analysis. Quackenbush states that most case studies focus on the extraction of a single feature, such as roads, which reduces the informative value of the resulting confusion matrix [72]. Furthermore, many studies report qualitative accuracy measures based on visual assessment. Those that provide more quantitative measures are often vague in describing their procedure [72]. Moreover, Foody states that quantitative measures are often misinterpreted and should therefore be interpreted with care. He argues that standardized measures and reporting schemes could be supportive, but are unlikely given the range of application fields and disciplines [217]. The ISPRS benchmark tests are a recent effort into this direction [220].

Furthermore, mixed pixels and registration problems might lead to a gap between extracted data and reference data. This results into low accuracy measures even for accurately extracted features [217]. A prioritization of a comprehensive cover of land plots over spatial accuracy is manifested in the fit-for-purpose land administration strategy proposed in [6]. The accuracy measure should therefore not only be interpreted with care, but also initially chosen with care taking into account the context and aim of the study as concluded in [217].

\subsection{Conclusion}

This review aimed to explore options to delineate boundaries for UAV-based cadastral mapping. At first, an initial review of cadastral mapping based on high-resolution optical sensor data was done to document the recent state-ofthe-art. Then, cadastral boundary concepts and characteristics were summarized. Thereafter, an extensive review was completed on methods that extract and assess linear features with boundary characteristics. Since cadastral features include a variety of objects, the methods could also be applied to detect linear features in further application fields. The workflow steps proposed for boundary delineation include pre-processing, image segmentation, line extraction, contour generation and post-processing. Per workflow step, the most popular methods were described and case studies that have proven their suitability were listed. The applicability of some representative methods on high-resolution UAV data was shown through their implementation on an exemplary UAV-derived orthoimage. In general, the workflow steps were supported in the majority of case studies and have proven to be valid when applied to UAV data. Thereafter, the most common accuracy assessment approaches were described. Moreover, advantages and drawbacks 
of each method were outlined, resulting in recommendations on their application for UAV-based cadastral mapping.

In conclusion, this review serves as a basis for the subsequent implementation of most suitable methods in a cadastral mapping workflow. Depending on the methods chosen and their implementation, different degrees of automation can be obtained. It would be possible to aim for a data-driven workflow that extracts visible boundaries, which then need to be processed by a human operator. Another option would be a model-driven workflow that delineates boundaries based on knowledge about their characteristics. Future work should focus on the automation of a suitable workflow. To increase the level of automation while reducing the amount of required human input is also a central aim of ongoing research [72]. Due to a lack of robustness of automatic feature extraction, some authors favor semi-automatic approaches that combine the interpretation skills of a human operator with the measurement speed of a computer [188,236]. Semi-automatic approaches that include editing capabilities seem indispensable for cadastral mapping approaches that focus on the participation of local stakeholders and the integration of local knowledge [6]. When evaluating an entire boundary delineation workflow for cadastral mapping, the following points proposed in [82] can be suggested as a basis for evaluation: The workflow should correctly and completely extract all relevant boundaries, be simple in parameterization with a high degree of automation and a minimal need of interaction, have a low computational effort, include self-assessment to increase reliability and be robust against varying quality of input data. The varying quality of input data might result from the application of different UAV platforms and sensors. Their influence on the choice of optimal workflow steps for cadastral mapping could be investigated in future work.

Overall, this review contributes to the applicability of UAVs, which according to Watts et al., has the potential to revolutionize remote sensing and its application fields to the same degree as the advent of Geographical Information Systems (GIS) did two decades ago [18]. For cadastral mapping, numerous studies have demonstrated the potential of UAVs especially in terms of fast data capturing and high accuracy [30]. UAV-based cadastral mapping could contribute to contemporary initiatives such as the United Nations' sustainable development goals, as it allows a new economic, environmental and social approach to cadastral mapping [237]. With UAVs being able to rapidly map small areas, the cadastral map could be kept up-to-date at low-cost in a sustainable way. These aspects together with the possibility of creating a transparent and participatory mapping process could contribute to another recent initiative, namely fit-for-purpose land administration published by the World Bank and the International Federation of Surveyors (FIG) [6]. 
Future work might concentrate on the integration of existing maps as a source of geometric and semantic information that was left undetected by the automatic feature extraction. Existing maps are incorporated in the workflow of cadastral mapping to support the manual delineation, as basis for map updates or for accuracy assessment $[11,13]$. Their potential to support the automatic feature extraction as proposed for road extraction [208] is not yet exploited and hardly investigated [238]. As a further data source, smart sketchmaps that transfer hand drawn maps into topologically and spatially corrected maps could be integrated in the feature extraction workflow [239]. This would allow integrating local spatial knowledge and to delineate socially perceived boundaries. Those boundaries are not visible to optical sensors and were excluded from this review. Furthermore, the boundary delineation methods could be enhanced to support the increasingly prominent area of 3D cadastral mapping of boundaries and buildings [74]. This would allow a detailed representation of complex interrelated titles and land uses [41]. Future development on UAV-based cadastral mapping can be expected since the ISPRS lists UAVs as key topic and stresses their potential for national mapping in their recent paper on trends and topics for future work [95]. Moreover, the European Union has acknowledge the use of UAV-derived orthoimages as a valid source for cadastral mapping and further applications [31]. 


\section{Contour Detection for UAV-based Cadastral Mapping*}

* This chapter is based on:

240. Crommelinck, S.; Bennett, R.; Gerke, M.; Yang, M.Y.; Vosselman, G. Contour Detection for UAV-Based Cadastral Mapping. Remote Sensing 2017, 9, 1-13. 


\begin{abstract}
Unmanned Aerial Vehicles (UAVs) provide a flexible and low-cost solution for the acquisition of high-resolution data. The potential of high-resolution UAV imagery to create and update cadastral maps is increasingly investigated. Existing procedures generally involve substantial fieldwork and many manual processes. Arguably, multiple parts of UAV-based cadastral mapping workflows could be automated. Specifically, as many cadastral boundaries coincide with visible boundaries they could be extracted automatically using image analysis methods. This study investigates the transferability of $\mathrm{gPb}$ contour detection, a state- of-the-art computer vision method, to remotely sensed UAV images and UAV-based cadastral mapping. Results show that the approach is transferable to UAV data and automated cadastral mapping: object contours are comprehensively detected at completeness and correctness rates of up to $80 \%$. The detection quality is optimal when the entire scene is covered with one orthoimage, due to the global optimization of $\mathrm{gPb}$ contour detection. However, a balance between high completeness and correctness is hard to achieve: a combination with area based segmentation and further object knowledge is proposed. The localization quality shows the usual dependency on ground resolution. The approach offers the potential to accelerate the process of general boundary delineation during the creation and updating of cadastral maps.
\end{abstract}




\subsection{Introduction}

Unmanned Aerial Vehicles (UAVs) have gained increasing popularity in remote sensing as they provide a rapid, low-cost and flexible acquisition system for high-resolution data [14,15,39]. Recently, cadastral mapping has emerged as a field of application for UAVs [38,241-243]. Cadastral maps show the extent, value, and ownership of land [57] and are considered crucial for a continuous and sustainable recording of land rights [7]. In contemporary settings, UAV data are employed both to create and to update cadastral maps, mostly through manual delineation of visible cadastral boundaries. An overview of case studies investigating the potential of UAVs for cadastral mapping and their approaches for boundary delineation is provided in [44]. However, none of the case studies described provide an automated approach for cadastral boundary delineation. Especially visible boundaries, manifested through physical objects, could potentially be extracted automatically [41]. A large number of cadastral boundaries are assumed to be visible, as they coincide with natural or manmade object contours [42,43]. Such visible boundaries might be extractable with computer vision methods that detect object contours in images.

\subsubsection{Contour Detection}

Contour detection refers to finding boundaries between objects or segments. Early approaches, such as Canny edge detection [171], extract edges by calculating gradients of local brightness, which are thereafter combined to contours. The approach typically detects irrelevant edges in textured regions. Later approaches include additional cues such as texture [244] and color [94] to identify contours. Maire et al., extended these approaches to consider multiple cues on both the local and global image scales through spectral partitioning [245]. Image information on a global scale allows for identification of contours not initially recognized by generating closed object outlines and eliminating irrelevant contours in textured regions. In [116,246], the closing of object outlines is provided by a hierarchical segmentation that partitions an image into meaningful objects. Detecting contours and assigning probabilities as presented in $[116,245,246]$ is referred to as gPb (globalized probability of boundary). The concept is summarized in Figure 3.1. The justification for using the method is based on [44], in which a workflow and feature extraction methods suitable for cadastral mapping are provided. gPb contour detection combines the workflow steps of image segmentation, line extraction and contour generation. 


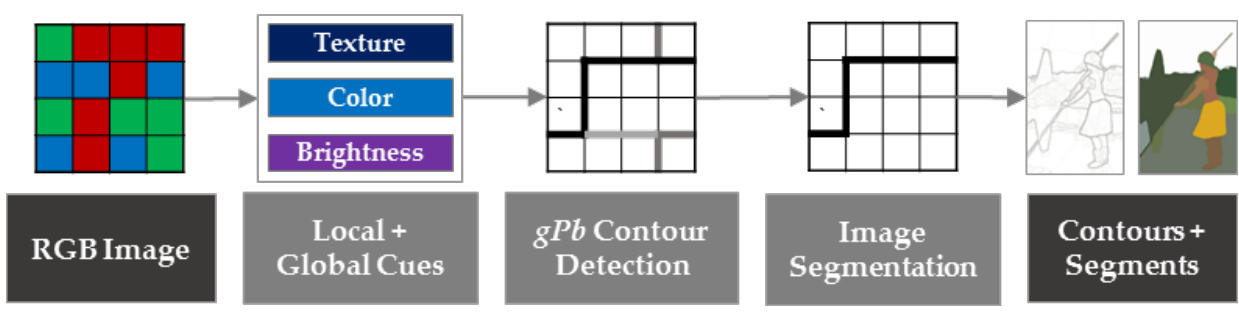

Figure 3.1. Combined gPb contour detection and hierarchical image segmentation for the delineation of closed object contours from RGB images described in [116]. The example image is taken from the 'Berkeley Segmentation Dataset and Benchmark' [247] and is processed with the publicly available source code for $\mathrm{gPb}$ contour detection [248].

In a first step of $\mathrm{gPb}$ contour detection, oriented gradient operators for brightness, color and texture cues are calculated by measuring their differences on two halves of a differently scaled disc. Results are stored in an affinity matrix that represents similarities between pixels. Small similarities, i.e., a strong oriented gradient, indicates a boundary between two distinct regions. Subsequently, all cues are merged based on a logistic regression classifier to predict the orientation and the posterior probability of a boundary, i.e., edge strength, at each image pixel. The image information on a global scale is obtained through spectral clustering. The local and global image scales convey different information: the former extracts all edges, while the latter extracts only the most salient edges. Both are combined through learning techniques as a weighted sum of local and spectral signals. This allows the assignment of a probability to each contour, resulting in the $\mathrm{gPb}$ contour detector.

The following step, i.e., image segmentation, consists of (i) an Oriented Watershed Transform (OWT) that forms initial regions from contours; and (ii) the construction of an Ultrametric Contour Map (UCM) that defines a hierarchical segmentation [249]. OWT is a variant of the watershed algorithm and constructs a set of initial regions from the oriented contour signal provided by a contour detector. UCM represents a hierarchy of these regions obtained by weighting each boundary and their agglomerative clustering. The image segmentation, consisting of the two steps OWT and UCM, can be applied to the output of any contour detector. However, it has been proven to work optimally on the output of the gPb contour detector [246].

The overall results are (i) a contour map, in which each pixel is assigned a probability for being a boundary pixel; and (ii) a binary boundary map, in which each pixel is labelled as either 'boundary' or 'no boundary' and from which closed segments can be derived. The number of contours transferred from the contour map to closed segments in the boundary map is defined by a threshold, which is referred to as scale $k$ in $[116,246]$ and in the following. The processing of $\mathrm{gPb}$-owt-ucm is referred to as $\mathrm{gPb}$ contour detection in this study. 
gPb contour detection provides accurate results compared to other approaches [116] and is often referred to as a state-of-the-art method for contour detection [250-252]. As the cue combination is learned, based on a large number of natural images from the 'Berkeley Segmentation Dataset and Benchmark' [247], the approach seeks to be transferable to images of different contexts. Nevertheless, gPb contour detection has hardly been applied to remotely sensed data $[253,254]$ and, to the best of the authors' knowledge, never to UAV data. The transferability of methods from computer vision to remote sensing is challenging, as both are often developed for image data with different characteristics: a benchmark dataset used in computer vision, such as the 'Berkeley Segmentation Dataset and Benchmark', contains natural images of maximal 1000 pixels in width and height, whereas a benchmark dataset used in remote sensing, such as the 'ISPRS Benchmark' [220], contains images from multiple sensors with higher numbers of pixels and larger Ground Sample Distances (GSD). This study addresses the transferability of gPb contour detection, originally designed to work on natural images, to remotely sensed UAV orthoimages.

\subsubsection{Objective and Organization of the Study}

This study investigates which processing is required for a state-of-the-art contour detection method from computer vision, namely $\mathrm{gPb}$ contour detection, to be applied to remotely sensed data of high-resolution, namely UAV data. Once the technical transferability is defined, the applicability of the method within the application field of cadastral mapping is investigated. This study aims to outline the potential of $\mathrm{gPb}$ contour detection for an automated delineation of cadastral boundaries demarcated by visible objects.

Overall, the study addresses the research gaps of transferring a method developed within computer vision to an application in remote sensing, where images have different characteristics. Further, it encounters the lack of automation within cadastral boundary delineation by investigating the applicability of gPb contour detection.

The paper is structured as follows: after having described the context of this research, the UAV datasets as well as the methodological approach are described. Then, the results are described and discussed. Concluding remarks include generic statements about the transferability and applicability of gPb contour detection for UAV-based delineation of visible cadastral boundaries.

\subsection{Materials and Methods}

\subsubsection{UAV Data}

Three UAV orthoimages of different extents showing rural areas in Germany, France and Indonesia were selected for this study. Rural areas were chosen 
because the number of visible boundaries is usually higher in rural areas compared to high-density urban areas. Table $\mathbf{3 . 1}$ shows specifications of the data capture, while Figure $\mathbf{3 . 2}$ shows orthoimages of the study areas.

Table 3.1. Specifications of UAV datasets per study area.

\begin{tabular}{|c|c|c|c|c|c|}
\hline Location & Acquisition & UAV Model & $\begin{array}{l}\text { GSD } \\
{[\mathrm{cm}]}\end{array}$ & Extent [m] & Pixels \\
\hline $\begin{array}{l}\text { Amtsvenn } \\
\text { Germany }\end{array}$ & Sept. 2016 & $\begin{array}{c}\text { GerMAP } \\
\text { G180 }\end{array}$ & 4.86 & $1000 \times 1000$ & $20,538 \times 20,538$ \\
\hline $\begin{array}{l}\text { Toulouse } \\
\text { France }\end{array}$ & Oct. 2016 & $\begin{array}{l}\text { DelairTech } \\
\text { DT18 }\end{array}$ & 3.61 & $500 \times 500$ & $13,816 \times 13,816$ \\
\hline $\begin{array}{c}\text { Lunyuk } \\
\text { Indonesia }\end{array}$ & Oct. 2015 & $\begin{array}{c}\text { DJI Phantom } \\
3 \text { Advanced }\end{array}$ & 3.00 & $250 \times 250$ & $8,344 \times 8,344$ \\
\hline
\end{tabular}

\subsubsection{Reference Data}

The study is based on the assumption that large portions of cadastral boundaries are visible [42]. Therefore, the method is intended to extract contours of physical objects that demarcate cadastral boundaries. A general list of such objects is rarely available in literature and strongly depends on the area of investigation [44]. From a list of objects provided in [44], the following objects were assumed to indicate cadastral boundaries for the investigated study areas: roads, fences, hedges, stone walls, roof outlines, agricultural field outlines as well as outlines of tree groups. The contours of these objects were manually delineated for all three orthoimages (Figure 3.3). The reference data is not identical with cadastral boundaries since a subset of these, i.e., visible boundaries, are considered in this study. Cadastral boundaries are assumed to be more regular than the outlines of visible objects delineated as reference data. A workflow for cadastral boundary delineation would need to contain a step in which extracted contours are generalized to be more likely to be cadastral boundaries. This study is not designed to provide such a complete workflow; it seeks to delineate object contours as a first workflow step.

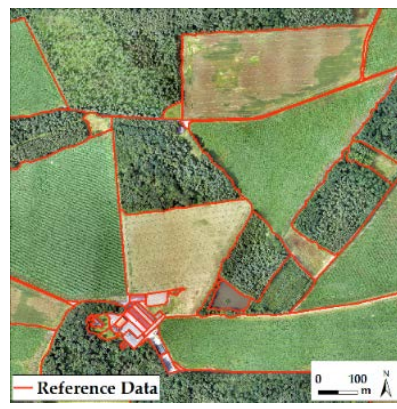

(a)



(b)

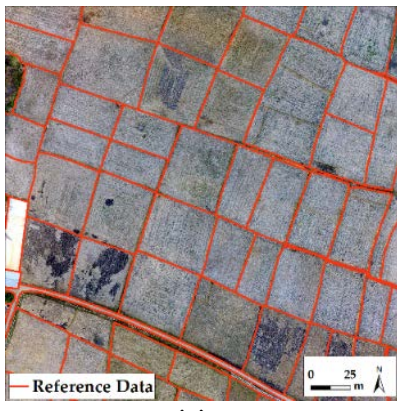

(c)

Figure 3.2. Manually delineated object contours used as reference data to determine the detection quality overlaid on UAV orthoimages of (a) Amtsvenn, Germany, (b) Toulouse, France, and (c) Lunyuk, Indonesia. 


\subsubsection{Image Processing Workflow}

The method investigated, gPb contour detection, is open source and available as a precompiled Matlab package [248]. This implementation was found to be inapplicable because of long computing time and insufficient memory when processing images of more than 1000 pixels in width and height. Therefore, an image processing workflow that reduces the original image size to $1000 \times 1000$ pixels was designed (Figure 3.3). The workflow consists of four steps, which are explained in the following. Apart from the Matlab implementation for $\mathrm{gPb}$ contour detection, all workflow steps were implemented in Python as QGIS processing scripts.

1) I mage Pre-processing: The UAV orthoimage was first resampled to lower resolutions ranging from 5 to $100 \mathrm{~cm}$ GSD. The resampling was based on nearest neighbor resampling, as it is computationally least expensive. Furthermore, the resampling to different GSDs enabled investigation of the influence of GSD in detecting object contours. The resampled images of 1000 to 5000 pixels in width and height were then tiled to tiles of $1000 \times 1000$ pixels. The smaller the GSD, the more tiles were created (Table 3.2).

2) Boundary Delineation: Then, gPb contour detection was applied to each tile of different GSDs. This resulted in contour maps containing probabilities for contours per pixel. By applying hierarchical image segmentation at scale $\mathrm{k}$ within the range $[0 ; 1]$, contours of a certain probability were closed and transferred to a binary raster map containing pixels for the classes 'boundary' and 'no boundary'. The resulting boundary map was created for all levels of $k$.

3) I mage Postprocessing: All tiles belonging to the same set were merged to one contour map and one binary boundary map, which was then vectorized.

4) Accuracy Assessment: The assessment was pixel-based and investigated the confusion matrix in terms of pixels labelled as True Positives (TP), True Negatives (TN), False Positives (FP) and False Negatives (FN) [228,255]. The accuracy assessment is designed to determine the accuracy in terms of (i) the detection quality, i.e., completeness and correctness as well as (ii) the localization quality, i.e., the accuracy of boundaries in a geometric sense:

(i) Each line was buffered with a radius distance of $2 \mathrm{~m}$ and converted to a raster format. The same buffering and rasterization was applied to the reference data. From the confusion matrix, the following errors were calculated: the error of commission within the range of [0; 100], showing the percentage of pixels erroneously labelled as 'boundary' and the error of omission within the range of $[0 ; 100]$, showing the percentage of pixels erroneously labelled as 'no boundary'. The 
generous buffer of $2 \mathrm{~m}$ was chosen in order to account for uncertainties in conjunction with manual delineation and resampling effects.

(ii) Since multiple objects, such as trees and bushes, do not provide exactly localizable contours, the localization accuracy requires a different set of reference data. Therefore, a subset of the reference data was evaluated containing exactly locatable object contours only, i.e., road and roof outlines. This subset was rasterized to a raster of $5 \mathrm{~cm}$ GSD and each reference line was buffered with distances from 0 $2 \mathrm{~m}$ at increments of $20 \mathrm{~cm}$. The binary boundary map was resampled to a GSD of $5 \mathrm{~cm}$ to be comparable to the reference raster. During the resampling, only one center pixel of $5 \times 5 \mathrm{~cm}$ was kept per pixel of a larger GSD to avoid having a higher number of pixels after resampling a boundary map of a larger GSD. The resampled binary boundary map was then compared to the reference raster. Based on the confusion matrix, the number of TPs per buffer zone was calculated to investigate the distance between TPs and the reference data and thus the influence of GSD on the localization quality.

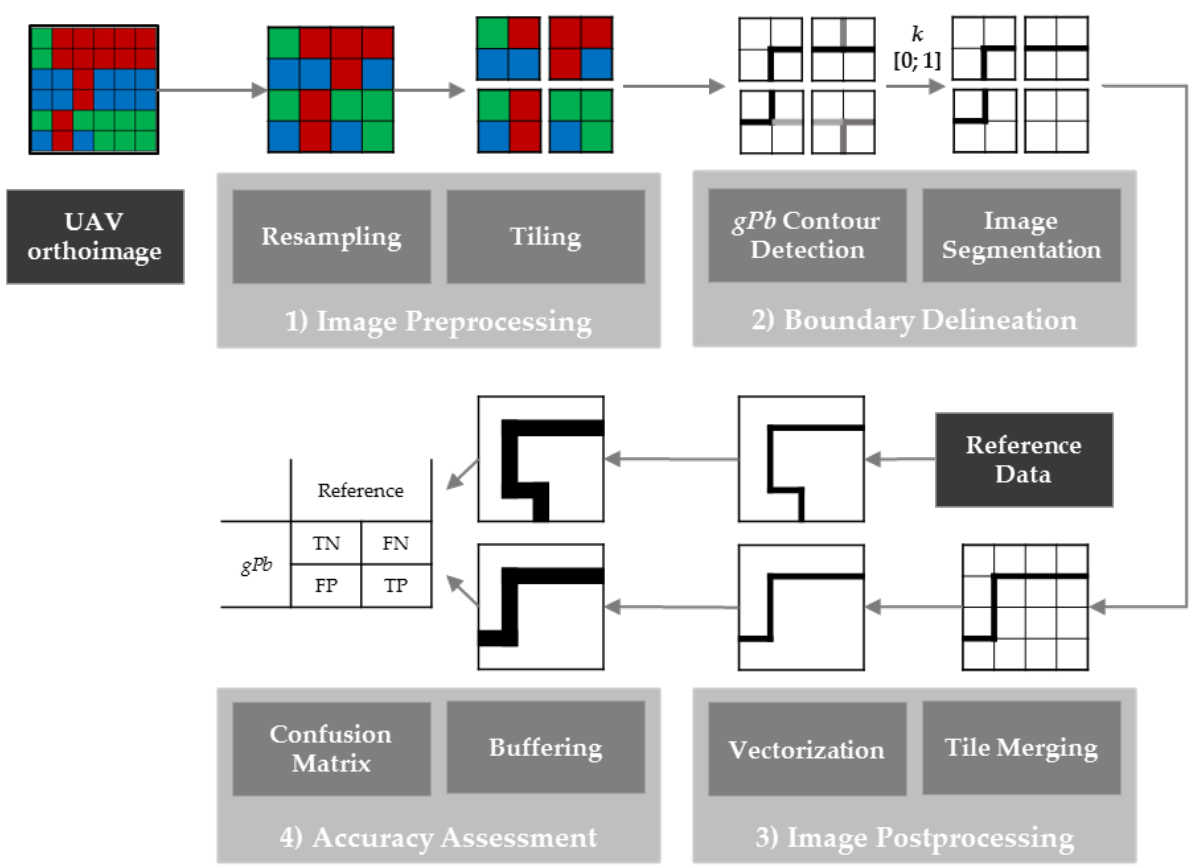

Figure 3.3. Image processing workflow for delineation of visual object contours from UAV orthoimages and its assessment based on the comparison to reference data.

\subsection{Results}

Resampling and tiling the UAV orthoimages to tiles of $1000 \times 1000$ pixels results in a higher number of tiles for images of a smaller GSD (Table 3.2). 
Applying gPb contour detection on each tile of $1000 \times 1000$ pixels belonging to the same set of tiles with an identical GSD results in a contour map and a binary boundary map (Figure 3.4). The lower the level of $k$, the fewer contours are transferred from the contour map to the binary boundary map (Figure 3.5). The processing time for each tile ranged from 10 to 13 minutes and was 11 minutes on average, with $\mathrm{gPb}$ contour detection running singlethreaded. The accuracy assessment is shown in terms of detection quality (Figure 3.6) and localization quality (Figure 3.7). To separate the influence of GSD and tiling on the detection quality, each untiled image of the largest GSD per study area was tiled to 25 tiles and assessed (Table 3.3).

Table 3.2. Number of Pixels and Ground Sample Distance (GSD) per tile after image preprocessing.

\begin{tabular}{ccccc}
\hline Pixels & Tiles & $\begin{array}{c}\text { GSD [cm] } \\
\text { Amtsvenn }\end{array}$ & $\begin{array}{c}\text { GSD [cm] } \\
\text { Toulouse }\end{array}$ & $\begin{array}{c}\text { GSD [cm] } \\
\text { Lunyuk }\end{array}$ \\
\hline $5000 \times 5000$ & 25 & 20 & 10 & 5 \\
$4000 \times 4000$ & 16 & 25 & 12.5 & 6.25 \\
$3000 \times 3000$ & 9 & 33 & 16.5 & 8.3 \\
$2000 \times 2000$ & 4 & 50 & 25 & 12.5 \\
$1000 \times 1000$ & 1 & 100 & 50 & 25 \\
\hline
\end{tabular}




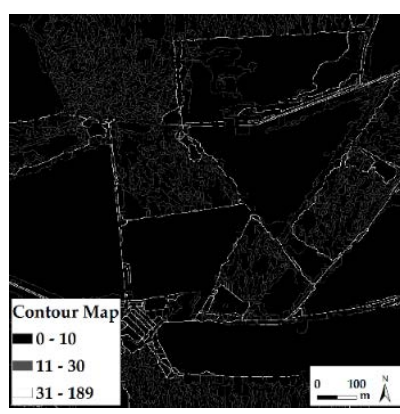

(a)

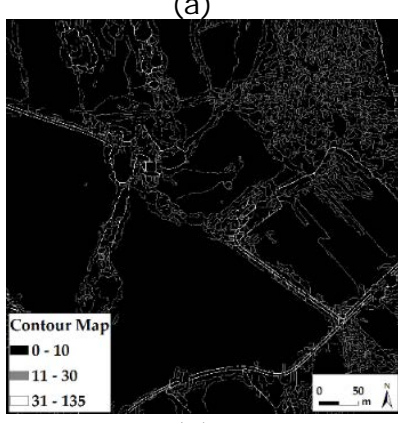

(d)



(g)

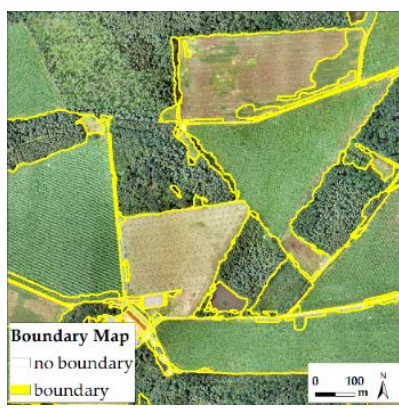

(b)

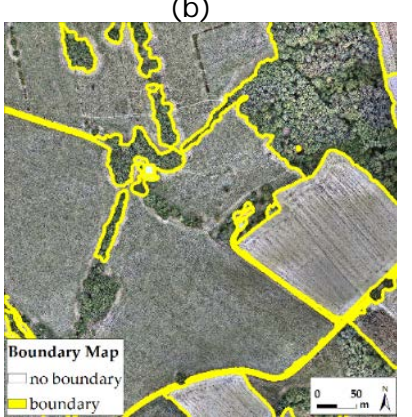

(e)

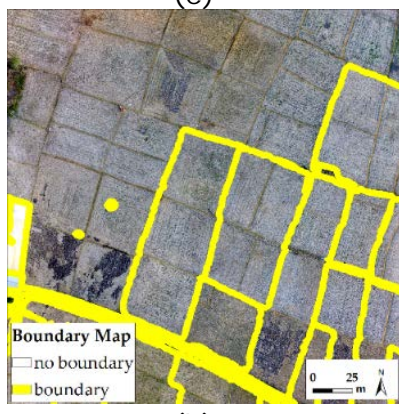

(h)

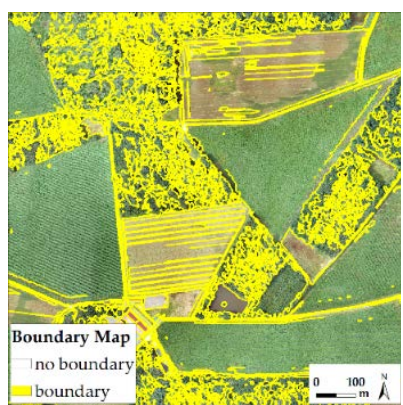

(c)

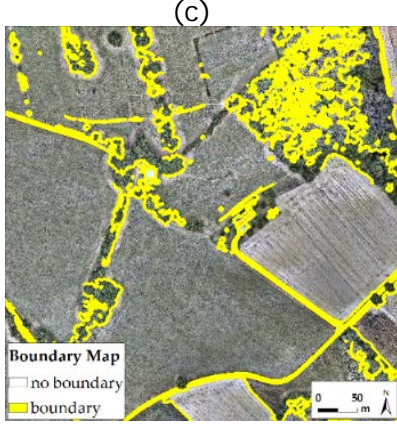

(f)



(i)

Figure 3.4. Examples of contour maps $(\mathbf{a}, \mathbf{d}, \mathbf{g})$ and binary boundary maps $(k=0.1)$ of Amtsvenn $(\mathbf{a}, \mathbf{b}, \mathbf{c})$, Toulouse $(\mathbf{d}, \mathbf{e}, \mathbf{f})$ and Lunyuk $(\mathbf{g}, \mathbf{h}, \mathbf{i})$. The boundary maps are buffered with $2 \mathrm{~m}$ to increase their visibility. (a, b, d, e, g, h) result from an untiled input image of $1000 \times 1000$ pixels, $(\mathbf{c}, \mathbf{f}, \mathbf{i})$ from an input image of $5000 \times 5000$ pixels merged from 25 tiles. 


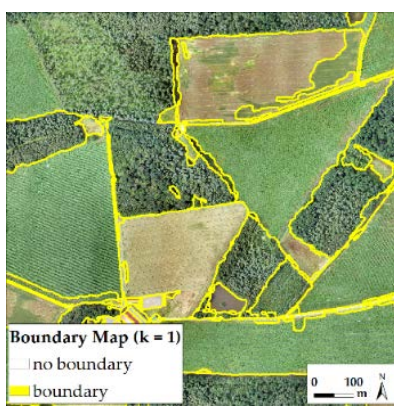

(a)

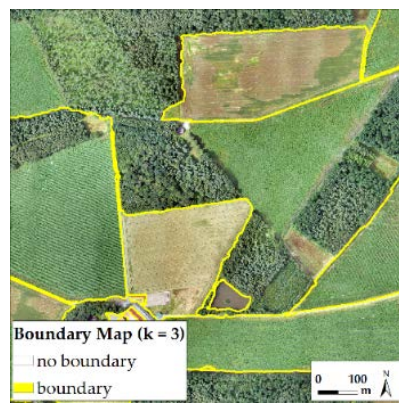

(b)



(c)

Figure 3.5. Binary boundary maps derived from untiled UAV orthoimage of Amtsvenn with a size of $1000 \times 1000$ pixels and $100 \mathrm{~cm}$ GSD at level (a) $k=0.1$, (b) $k=0.3$, and (c) $\mathrm{k}=0.5$.

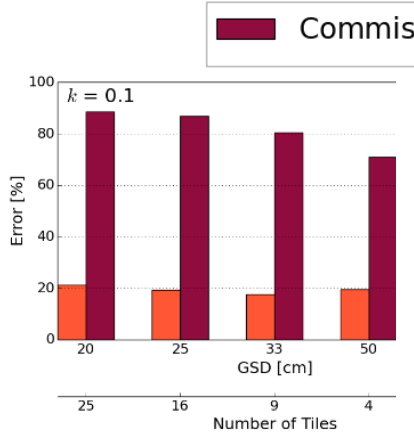

(a)

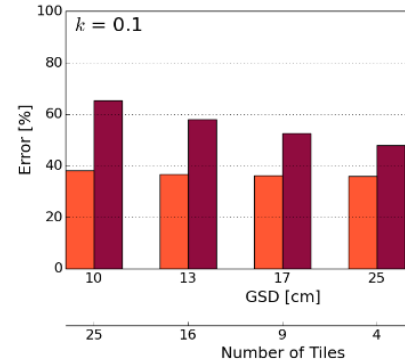

(b)

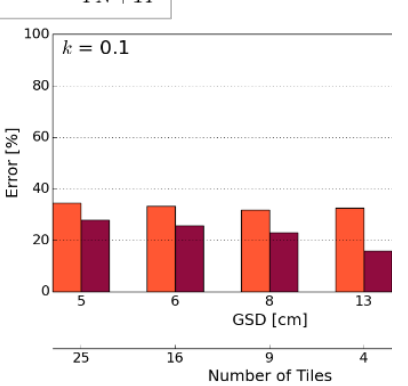

(c)

Figure 3.6. Detection quality: the errors of commission and omission is shown for binary boundary maps of different Ground Sample Distances (GSD) derived for (a) Amtsvenn, (b) Toulouse, and (c) Lunyuk at level $k=0.1$.



(a)

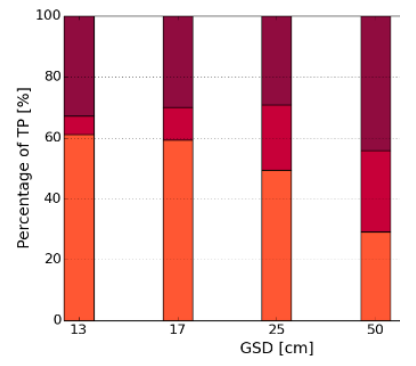

(b)



(c)

Figure 3.7. Localization quality: the distance between pixels being True Positives (TP) and the reference data relative to the total number of TPs per Ground Sample Distance (GSD) is shown for (a) Amtsvenn, (b) Toulouse, and (c) Lunyuk at level k = 0.1. 
Table 3.3. Comparison of detection quality for images of largest Ground Sample Distance (GSD) per study area for the untiled image and the same image merged from 25 tiles. Lower errors are marked in bold.

\begin{tabular}{|c|c|c|c|c|c|c|}
\hline \multirow{3}{*}{$\begin{array}{r} \\
\text { Pixels; GSD[cm] } \\
\text { Tiles }\end{array}$} & \multicolumn{2}{|c|}{ Amtsvenn } & \multicolumn{2}{|c|}{ Toulouse } & \multicolumn{2}{|c|}{ Lunyuk } \\
\hline & $1000 x$ & $0 ; 100$ & $1000 x$ & $00 ; 50$ & $1000 x$ & $00 ; 25$ \\
\hline & 1 & 25 & 1 & 25 & 1 & 25 \\
\hline $\begin{array}{r}\text { Error of } \\
\text { commission [\% ] }\end{array}$ & 55.15 & 70.01 & 23.43 & 53.88 & 17.21 & 31.10 \\
\hline $\begin{array}{r}\text { Error of } \\
\text { omission [\%] } \\
\end{array}$ & 13.44 & 68.75 & 27.44 & 90.12 & 52.30 & 96.24 \\
\hline
\end{tabular}

\subsection{Discussion}

\subsubsection{Detection Quality}

The error of commission, i.e., excessive detection, increases with parallel decreasing of GSD and increasing of tiles and is mostly lowest for the untiled input image regardless of scale $\mathrm{k}$ (Figure 3.6). Both the errors of commission and omission are higher when applying $\mathrm{gPb}$ contour detection on the tiled image compared to the untiled image of the same GSD (Table 3.3). Therefore, the detection quality depends on the number of tiles, i.e., the area of the captured scene, rather than the GSD for the three study areas: gPb contour detection considers the global extent of one image or one single tile, respectively. When taking a small tile of the UAV orthoimage into account, the global optimization is not efficient. This results in a higher number of contours detected in textured regions (oversegmentation). Therefore, more boundaries are detected in Figure $\mathbf{3 . 4 c / f / i}$, which consist of 25 tiles compared to Figure $\mathbf{3 . 4 b} / \mathbf{e} / \mathbf{h}$, which consist of one single tile. Overall, the global optimization works optimally if the entire scene is covered in one orthoimage.

The error of omission, i.e., missed boundaries, varies less than the error of commission per binary boundary map. It can be concluded that a higher number of tiles that cover smaller parts of the entire scene, does not lead to an increase in the error of omission (Figure 3.6). The lowest number of errors of omission are obtained for $k=0.1$ since fewer boundaries are missed in the over-segmented binary boundary map (Figure 3.5).

The overall detection accuracy is close to $100 \%$ since many pixels are classified correctly as 'no boundary'. It is therefore not visualized in Figure 3.6. A low level of $k$ leads to an oversegmentation of the image, while a higher level of $k$ leads to an undersegmentation or even the case that no boundaries are contained in the binary boundary map (Figure 3.5), which influences the errors of commission and omission accordingly. However, even for the lowest level of k, contours indicated in the contour map (Figure $\mathbf{3 . 4 9}$ ), might not be transferred to the binary boundary map (Figure $\mathbf{3 . 4 h}$ ). This indicates that when aiming for a high completeness of detected contours, which is considered 
optimal in [256] before integrating user interaction, the contour map should be considered for further processing.

The results for Amtsvenn show the highest number of errors of commission, due to many textured regions in which boundaries are erroneously detected. The error of commission is lowest for Lunyuk since the image contains rarely any textured regions or small objects. The high errors of omission for the Toulouse and Lunyuk data reveal that boundaries are less definite and visible in these images.

\subsubsection{Localization Quality}

The number of TPs within $20 \mathrm{~cm}$ distance to the reference data relative to the total number of TPs per GSD decreases for larger GSDs, for all study areas (Figure 3.7). For GSDs of $20-33 \mathrm{~cm}$ (Amtsvenn) and $10-25 \mathrm{~cm}$ (Toulouse and Lunyuk), the number of TP localized within $20 \mathrm{~cm}$ distance from the reference data ranges between $50-60 \%$. This percentage decreases for all study areas when the GSD is increased to $100 \mathrm{~cm}$. The results indicate that contours are more accurately localized for UAV images of a higher resolution.

\subsubsection{Discussion of the Evaluation Approach}

The study results strongly depend on the applied buffer distance. For detection quality, the buffer distance of $2 \mathrm{~m}$ was chosen. This does not represent the following two visually observed cases: (i) some boundaries run along the shadow of an object and are therefore shifted compared to the reference data that runs along the actual object contour. (ii) Some boundaries are covered by other objects, e.g., trees covering streets. Merging contours of smaller objects with the applied buffer distance does not represent such cases. Such issues could be resolved with an object detection that includes semantics, i.e., knowledge about the objects to be extracted. UAV-based approaches offer the potential to extract such object knowledge through incorporation of highresolution imagery, pointclouds and Digital Surface Models (DSM). According to Mayer, the use of such additional information makes object extraction more robust and reliable [256]. The approach to detection quality is employed similarly in other studies $[228,255,257,258]$. The authors argue that despite its strong dependency on the buffer size and its focus on positional accuracy while neglecting factors such as topological accuracy, the buffer approach provides a simple and comprehensive accuracy measure. Further, it can be used on both a vector and a raster representation and is easy to implement [258].

Apart from the accuracy assessment method, the manually drawn reference data strongly influences the results. Manually drawn reference data are argued to be valid for measuring the degree to which an automated system, as 
proposed in this study, outperforms a human operator [256]. However, each human might draw different reference data. Averaging a large number of manually drawn reference data, as proposed in [94], might reduce errors produced by an individual.

\subsubsection{Transferability and Applicability of $\mathrm{gPb}$ for Boundary Delineation}

gPb contour detection appears to be transferable to UAV orthoimages. The approach can support the automation of cadastral boundary delineation in cases where cadastral maps are scarcely available and concepts such as fitfor-purpose and responsible land administration are in place [6,259]. Such concepts accept general boundaries, for which the positional correctness is of lower importance [42]. In cases where a map needs to be created or updated, and positional inaccuracy is tolerated to a certain degree, editing automatically generated visible boundaries from gPb contour detection on an UAV orthoimage might be less cost- and time-intensive than manually delineation all boundaries. This would need to be verified by comparing both cadastral mapping workflows as a whole. Hence, future work is required to determine to which degree the object contours coincide with cadastral boundaries and which level of accuracy is required to outperform a manual cadastral mapping workflow. For road extraction, which is closely related to the object detection of this study, Mayer et al. propose a correctness of around $85 \%$ and a completeness of around $70 \%$ for an approach to be of real practical importance [232]. Such values can hardly be achieved when applying solely gPb contour detection for cadastral boundary delineation.

Furthermore, there might be cases in which only a small portion of cadastral boundaries is visible or object contours do not coincide with cadastral boundaries. Then, the proposed data-driven approach will need to be combined with a knowledge-driven approach. To reliably delineate a closed and geometrically and topologically correct network of boundaries, further object knowledge should be incorporated, e.g., through semi-supervised machine learning approaches and thus derived complementary data. The contour map containing the probability for each contour detected and for which the level of $k$ does not need to be defined, could be employed as a first workflow step. The salient contours detected in this step could be balanced by incorporating an area-based segmentation, resulting in more homogeneous areas. Adding further steps to the workflow could generate an output directly comparable to cadastral boundaries. In future, the authors aim to develop a workflow that remains as automatic, generic and adaptive to different scenarios as possible, similarly formulated in [260] as a need for contemporary boundary detection schemes. 


\subsection{Conclusion}

This study contributes to the recent endeavor of making the process of cadastral mapping more reproducible, transparent, automated, scalable and cost-effective. This is achieved by proposing the application of UAV orthoimages combined with automated image analysis, i.e., a state-of-the-art computer vision method that has never been applied to UAV data. The approach does not require prior knowledge (learning) and automatically detects object contours from UAV orthoimages that indicate visible cadastral boundaries. More specifically, this study investigates the transferability of gPb contour detection to UAV images and its applicability for automated cadastral mapping. This is investigated in terms of detection and localization quality for three different study areas.

The approach is most suitable for areas in which object contours are clearly visible and coincide with cadastral boundaries. The approach proposed offers potential to simplify and accelerate the cadastral mapping process. This applies to the automated outlining of general boundaries during the creation and updating of cadastral maps. To establish the comparability of the detected object contours with cadastral boundaries, future work will focus on incorporating the approach proposed here with machine learning methods to integrate further object knowledge. The goal is to generate a tool for cadastral boundary delineation that is highly automatic, generic and adaptive to different scenarios. 


\section{SLIC Superpixels for Object Delineation from UAV Data*}

* This chapter is based on:

261. Crommelinck, S.; Bennett, R.; Gerke, M.; Koeva, M.; Yang, M.Y.; Vosselman, G. In SLIC Superpixels for Object Delineation from UAV Data, International Conference on Unmanned Aerial Vehicles in Geomatics, Bonn, Germany, 04-07 September, 2017; IV-2/W3, pp 9-16. 


\begin{abstract}
Unmanned aerial vehicles (UAV) are increasingly investigated with regard to their potential to create and update (cadastral) maps. UAVs provide a flexible and low-cost platform for high-resolution data, from which object outlines can be accurately delineated. This delineation could be automated with image analysis methods to improve existing mapping procedures that are cost, time and labor intensive and of little reproducibility. This study investigates a superpixel approach, namely simple linear iterative clustering (SLIC), in terms of its applicability to UAV data. The approach is investigated in terms of its applicability to high-resolution UAV orthoimages and in terms of its ability to delineate object outlines of roads and roofs. Results show that the approach is applicable to UAV orthoimages of $0.05 \mathrm{~m}$ GSD and extents of 100 million and 400 million pixels. Further, the approach delineates the objects with the high accuracy provided by the UAV orthoimages at completeness rates of up to $64 \%$. The approach is not suitable as a standalone approach for object delineation. However, it shows high potential for a combination with further methods that delineate objects at higher correctness rates in exchange of a lower localization quality. This study provides a basis for future work that will focus on the incorporation of multiple methods for an interactive, comprehensive and accurate object delineation from UAV data. This aims to support numerous application fields such as topographic and cadastral mapping.
\end{abstract}




\subsection{Introduction}

Superpixel approaches, introduced in [262], group pixels into perceptually meaningful atomic regions. Superpixels are located between pixel- and objectlevel: they carry more information than pixels by representing perceptually meaningful pixel groups, while not comprehensively representing image objects. Superpixels can be understood as a form of image segmentation that oversegment the image in a short computing time. Comparisons to similar approaches that can be found in [114,263-267] have demonstrated their advantages: The outlines of superpixels have shown to adhere well to natural image boundaries, as most structures in the image are conserved [262,264]. Furthermore, they allow reducing the susceptibility to noise and outliers as well as to capture redundancy in images. With image features being computed for each superpixel rather than each pixel, subsequent processing tasks are reduced in complexity and computing time. Thus, superpixels are considered useful as a preprocessing step for analyses at object level such as image segmentation [114,268].

In general, the success of image segmentation activities is highly variable as it depends on the image, the algorithm and its parameters: an algorithm that performs as desired on one image might result in a lower segmentation quality when applied with the same parameters to another image. This study investigates the applicability of a superpixel approach, namely simple linear iterative clustering (SLIC), in terms of its ability to delineate object outlines of roads and roofs from UAV data. The approach has proven to accurately delineate object outlines [114]. In this study, SLIC is applied to two UAV orthoimages of $0.05 \mathrm{~m}$ GSD and extents of 100 million and 400 million pixels.

Object delineation is potentially useful in numerous application fields, such as topographic and cadastral mapping [44]. Cadastral mapping refers to mapping the extent, value, and ownership of land, being crucial for a continuous and sustainable recording of land rights [7]. Cadastral mapping is used in this study as an example application field to investigate the applicability of SLIC superpixels for an automatic delineation of object outlines. Such visible outlines can correspond to cadastral boundaries, as a large portion of cadastral boundaries are assumed to be visible [42]. Automatically delineating visible boundaries, would thus improve cadastral mapping approaches in terms of cost, time, accuracy and reproducibility. This study investigates SLIC superpixels as part of a boundary delineation workflow. The study does not provide a full workflow for automatic delineation of visible cadastral boundaries. However, when used alongside other more conventional mapping techniques, the approach may improve the time and costs associated with wide-area cadastral mapping projects. 


\subsection{Related Work}

\subsubsection{Superpixel Approaches}

Superpixels oversegment an image by forming compact and uniform groups of pixels that have similar characteristics in, e.g., color or geometry. In the past, multiple superpixel approaches have been developed. They can be classified into i) graph-based and ii) gradient-ascent-based approaches:

- In i), each pixel is considered a node in a graph. An edge weight is defined between all pairs of nodes that is proportional to their similarity. Then, a cost function defined on the graph is formulated and minimized, in order to extract superpixel segments. Examples of graph-based approaches are $[112,269,270]$.

- In ii), pixels are iteratively mapped to a feature space to delineate denser regions that represent clusters. Each iteration refines each cluster to obtain a better segmentation until convergence. Examples of gradient-ascentbased approaches are [271-273].

State-of-the-art superpixel approaches have been compared in [114,263-267] considering speed, memory efficiency, compactness of outlines, their ability to adhere to image boundaries and their impact on segmentation performance. Boundary adherence is often measured via boundary recall, indicating how many true edges are missed, and via undersegmentation, indicating to what extent superpixels exceed outlines of the reference data [114,264]. The SLIC superpixel approach, belonging to the group of gradient-ascent-based approaches, appears as the best overall performer: the algorithm is low in processing time and produces compact and nearly uniform superpixels that are positively evaluated in terms of boundary recall and undersegmentation error [114,263-267].

\subsubsection{SLIC Approach}

SLIC was introduced in [268] and later extended to a zero parameter version of SLIC called SLICO and compared to state-of-the-art superpixel approaches in [114]. SLIC considers image pixels in a 5D space, defined by the L*a*b values of the CIELAB color space as well as their $x$ and $y$ coordinates. Pixels in the 5D space are clustered based on an adapted k-means clustering integrating color similarity and proximity in the image plane. The clustering is based on a distance measure $D$ that measures color similarity in L*a*b space $\left(d_{c}\right)$ and pixel proximity in $\mathrm{x}$, y space $\left(d_{s}\right)$. The latter is normalized by a grid interval (S) that defines the square root of the total number of image pixels divided by the number of superpixels $(\mathrm{k})$. The compactness and regularity of the superpixels is controlled with the constant $\mathrm{m}$. This parameter functions as a weighting 
criteria between the spatial distance $\left(d_{c}\right)$ and the spectral distance $\left(d_{s}\right)$. A larger $\mathrm{m}$, increases the weight of spatial proximity, which leads to more compact superpixels with boundaries adhering less to spectral outlines in the image.

$$
\mathrm{D}=\sqrt{\left(\frac{d_{c}}{m}\right)^{2}+\left(\frac{d_{s}}{s}\right)^{2}}
$$

SLICO replaces the constant values for $m$ and $S$ used in (1) to normalize spectral and spatial proximity, by iteratively normalizing their proximity. The proximities are dynamically normalized for each cluster considering the maximum observed spectral distance $\left(m_{c}\right)$ and spatial distance $\left(m_{s}\right)$ from the previous iteration. This leads to a more consistent superpixel compactness and a reduced need to define parameters.

$$
\mathrm{D}=\sqrt{\left(\frac{d_{c}}{m_{c}}\right)^{2}+\left(\frac{d_{s}}{m_{s}}\right)^{2}}
$$

In general, only pixels within D are considered during clustering, which makes SLIC fast and computational efficient compared to conventional k-means clustering. Another advantage of SLIC is its ability to be applied to images greater than 0.5 million pixels, processing time scales linearly with the number of pixels. Further, it is simple to implement and demands low computational and memory cost. The boundary recall is lowest compared to other approaches. However, the risk of losing meaningful image edges remains, when an edge is placed inside a superpixel [114].

SLIC implementations are available in OpenCV [274], VLFeat [275], GDAL [276], Scikit [277], Matlab [278] and GRASS [279]. They are mostly based on the two SLIC versions proposed in [114]. For the first version (SLIC), the parameter $\mathrm{k}$ specifies the number of approximately equally sized superpixels. Optionally, the compactness parameter $\mathrm{m}$ can be set to control the trade-off between superpixels' homogeneity and boundary adherence. This version generates regular-shaped superpixels in untextured regions and highly irregular superpixels in textured regions (Figure 4.1a). For the second version (SLICO), only the parameter $\mathrm{k}$ can be defined, while $\mathrm{m}$ is adaptively refined for each superpixel. SLICO generates regular-shaped superpixels across the scene, regardless of texture (Figure 4.1b) [114]. 


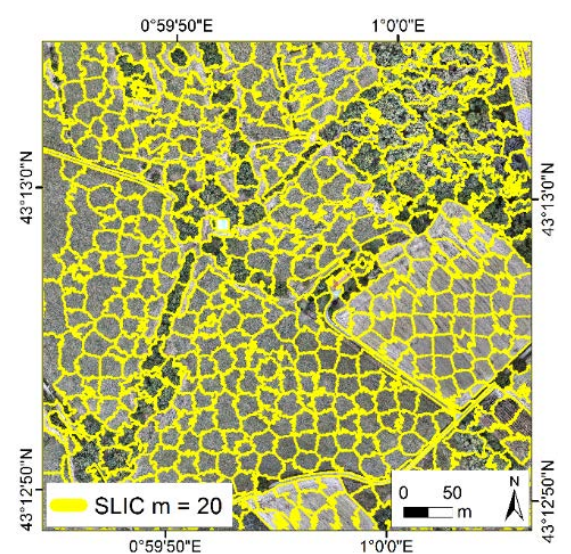

(a)

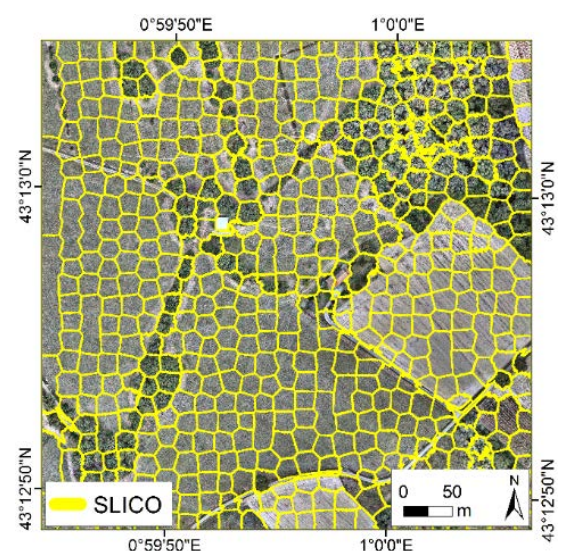

(b)

Figure 4.1. (a) SLIC $(m=20)$ and (b) SLICO applied to an UAV orthoimage of Toulouse with $0.05 \mathrm{~m}$ ground sample distance (GSD) and $\mathrm{k}=625$. SLIC generates regular-shaped superpixels in untextured regions and highly irregular superpixels in textured regions. SLICO generates regular-shaped superpixels across the scene, regardless of texture. SLICO superpixels are spatially more compact, but spectrally more heterogeneous.

\subsubsection{Superpixels in Remote Sensing}

The benefits of analyzing groups of pixels instead of single pixels, has been verified from a computer vision perspective for multiple applications such as object recognition $[280,281]$. This has similarly been done from a remote sensing perspective for object-based image analysis (OBIA) [78]. The use of superpixels in computer vision is increasingly popular, whereas only few studies in remote sensing consider superpixels [263,282-288].

However, the need and acceptance of superpixels in remote sensing is presumed: the local spatial autocorrelation between pixels with a highresolution in remotely sensed imagery is high. Thus one object is often composed of many pixels with similar characteristics [289]. This has led to the formulation of the OBIA paradigm [78]. Superpixels that group pixels of similar characteristics into an oversegmented image are considered a preprocessing step in conventional OBIA approaches [282].

A comparison of four state-of-the-art superpixel approaches, with SLIC being the best choice considering speed and accuracy, has been conducted on satellite imagery of 0.5-0.6 m GSD and an extent of 4 million pixels [263]. In further studies that apply superpixels on remote sensing data, SLIC is equally considered as the most suitable superpixel approach $[283,285,287,290]$. SLIC has rarely been applied to UAV data, or for object delineation in topographic or cadastral mapping. This study aims to bridge both of these research gaps. 


\subsubsection{SLIC Superpixels for Object Delineation}

In general, SLIC cannot be considered as a standalone approach for object delineation. Each superpixel needs to be closed even if no object outline is available within the image. The larger $k$, the more outlines are generated that do not align with object outlines. In order to eliminate those unwanted outlines, SLIC could be combined with further segmentation methods. Another option, proposed in [287] would be to fuse neighboring SLIC regions of similar color to eliminate non-relevant outlines.

Combining the information from multiple segmentations has been investigated in other studies aiming to develop a transferable approach with a constant object recognition robustness and a reduced need for parameter optimization. Object outlines delineated through multiple segmentations are shown to be more reliable and robust compared to those detected by fewer segmentations $[280,281,291,292]$. This idea can equally be transferred to superpixels: combining superpixels with the output of a contour detector has shown to better delineate object contours, compared to using a standalone superpixel approach [272,293-295].

SLIC superpixels are often combined with the $\mathrm{Pb}$ [94] or the $\mathrm{gPb}$ [245] contour detector. These approaches combine texture, color and brightness to calculate probabilities of boundaries $(\mathrm{Pb})$ and globalized probabilities of boundaries $(\mathrm{gPb})$, respectively. The former considers these cues on a local scale, while the latter considers them on both a local and a global scale. Detected contours and superpixel outlines are mostly combined with cost functions that minimize the inter-class similarity while maximizing the intra-class similarity. The functions are optimized through learning based on computer vision benchmark datasets [293-295]. These functions are not directly transferable to remote sensing imagery, which have more complex characteristics. However, the approach of $\mathrm{gPb}$ contour detection has been investigated as a standalone approach for UAVbased cadastral mapping in [240]. The study shows that the approach provides a comprehensive initial detection of candidate objects that could be verified and located exactly by integrating SLIC outlines.

When combining SLIC and a further segmentation approach, such as gPb contour detection, moderate errors of omission are acceptable: outlines missed by SLIC might be detected by the second approach. In general, a low error of omission, i.e., a high level of completeness, is of utmost importance for an automated object detection system before integrating user interaction and thus reducing the system's automation [256]. The user interaction required to manually delineate a missed boundary (error of omission) is more timeconsuming than to delete an erroneously included boundary (error of 
commission). The goal is to minimize the summated time for editing both the error of omission and commission.

\subsection{Materials and Methods}

\subsubsection{UAV Data}

Two UAV orthoimages of different extents showing rural areas in Germany and France were selected for this study. Table $\mathbf{4 . 1}$ shows specifications of the data capture. Figure $\mathbf{4 . 2}$ shows the orthoimages of both study areas.

Table 4.1. Specifications of UAV datasets.

\begin{tabular}{rcc}
\hline Location & Amtsvenn & Toulouse \\
Country & Germany & France \\
\hline UAV model & GerMAP G180 & DT18 PPK \\
camera/focal length & Ricoh GR/18.3 & DT-3Bands RGB/5.5 \\
forward/sideward overlap [\%] & $80 / 65$ & $80 / 70$ \\
GSD [m] & 0.05 & 0.05 \\
extent [m] & $1000 \times 1000$ & $500 \times 500$ \\
\hline
\end{tabular}

\subsubsection{Reference Data}

Automatically delineating objects is considered useful for cadastral mapping, as object outlines often align with visible cadastral boundaries [42]. Examples for such objects are roads, fences, hedges and stone walls, as well as outlines of roofs, agricultural fields and tree groups [44]. From this list, road and roof outlines were selected for this study, as these are the objects with the highest visibility and the most accurately delineable outlines for both study areas. These outlines were manually delineated for parts, where the outlines could be localized exactly. Parts of road and roofs without a precisely distinguishable outline were not delineated as reference data (Figure 4.2). 


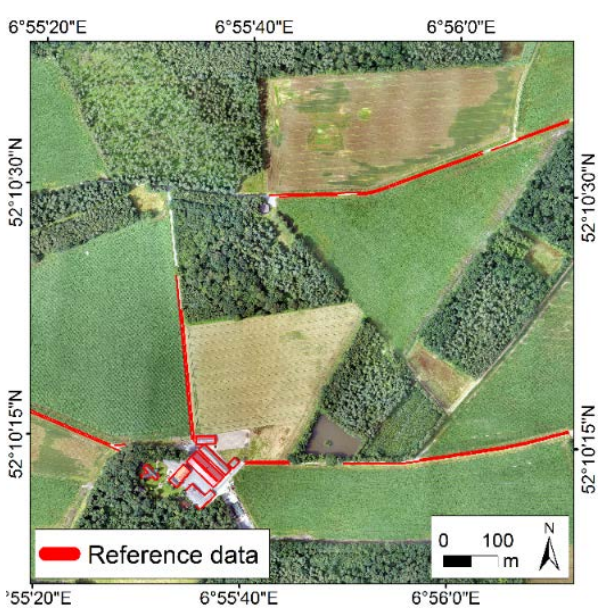

(a)

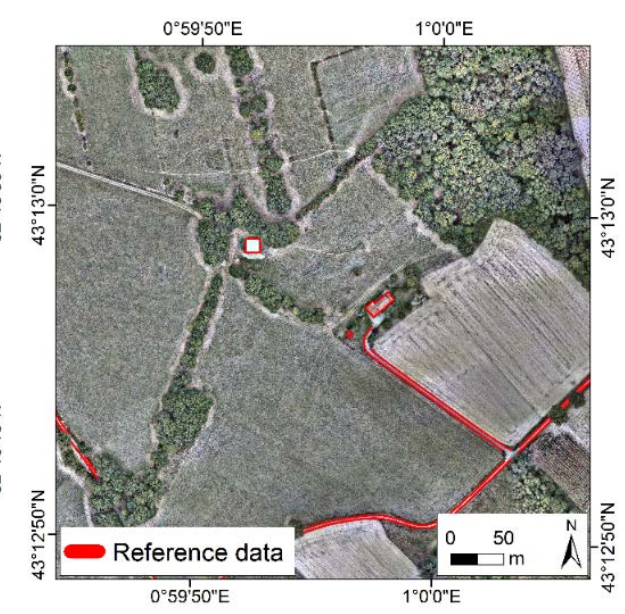

(b)

Figure 4.2. Manually delineated outlines of exactly localizable roads and roofs used for the accuracy assessment overlaid on UAV orthoimages of (a) Amtsvenn in Germany and (b) Toulouse in France. Outlines in close spatial proximity, such as two parallel outlines of roads, might appear as a thicker line, as they consist of two parallel lines in the reference data.

\subsubsection{Image Processing Workflow}

The image processing workflow consists of the application of SLIC on the UAV datasets and its accuracy assessment. For the SLIC application, a Matlab implementation was used [278], which is based on [114]. All further workflow steps were implemented in Python as QGIS processing scripts making use of functionalities from QGIS [296], GRASS [297] and GDAL [298].

\subsubsection{SLIC Application}

The Matlab implementation, used in this study, provides a SLIC and a SLICO version [278]. SLICO requires a predefined number of superpixels $k$, while SLIC requires $k$, as well as a compactness parameter $m$ that regularized the SLIC outlines. $\mathrm{k}$ was chosen in accordance to possible sizes of objects of interest in the range $[1 ; 400] \mathrm{m}^{2} . \mathrm{m}$ was chosen in accordance to recommendations from MathWorks in the range $[1 ; 20]$. Due to the different extents of the two UAV orthoimages (Table 4.1), this resulted in different numbers for $\mathrm{k}$ ranging from 625 to 1,000,000: the smaller the size of one superpixels, the larger the total number of superpixels $k$ (Table 4.2). SLIC was applied to the entire orthoimage. 
Table 4.2. Varying numbers of superpixels $k$ resulting for the two study areas with a coverage of $1,000,000 \mathrm{~m}^{2}$ (Amtsvenn) and 250,000 $\mathrm{m}^{2}$ (Toulouse).

\begin{tabular}{cccc}
\hline GSD per superpixel $[\mathrm{m}]$ & $\begin{array}{c}\text { Coverage per } \\
\text { superpixel }\left[\mathrm{m}^{2}\right]\end{array}$ & $\begin{array}{c}\mathbf{k} \\
\text { Amtsvenn }\end{array}$ & $\begin{array}{c}\mathbf{k} \\
\text { Toulouse }\end{array}$ \\
\hline 1 & 1 & $1,000,000$ & 250,000 \\
2 & 4 & 250,000 & 62,500 \\
3 & 9 & 111,111 & 27,778 \\
4 & 16 & 62,500 & 15,625 \\
5 & 25 & 40,000 & 10,000 \\
10 & 100 & 10,000 & 2,500 \\
15 & 225 & 4,444 & 1,111 \\
20 & 400 & 2,500 & 625 \\
\hline
\end{tabular}

\subsubsection{Accuracy Assessment}

In order to decrease the processing time of the accuracy assessment, the SLIC outlines were clipped to a buffer of $0.3 \mathrm{~m}$ radius around the reference data. Then, all lines in the reference data and the clipped SLIC outlines were buffered with a radius of $0.1 \mathrm{~m}$. These datasets were converted to a raster format of $0.05 \mathrm{~m}$ pixel size. Then, each SLIC dataset was overlaid with the reference data, in order to label each pixel as true positive (TP), true negative (TN), false positive (FP) or false negative (FN). The sum of pixels with an identical label was summarized in a confusion matrix. From the confusion matrix, the error of omission (3) and the error of commission (4) was calculated in the range $[0 ; 100]$ :

$$
\begin{aligned}
& \text { error of omission }[\%]=\frac{\mathrm{FN}}{\mathrm{FN}+\mathrm{TP}} * 100 \\
& \text { error of commission }[\%]=\frac{\mathrm{FP}}{\mathrm{FP}+\mathrm{TP}} * 100
\end{aligned}
$$

The error of omission captures the percentage of pixels erroneously labelled as 'no outline', i.e., the percentage of object outlines that are missed by the SLIC outlines. The error of commission captures the percentage of pixels erroneously labeled as 'outline', i.e., the percentage of object outlies that are incorrectly included in the SLIC outlines. These measures are based on [258] and evaluate to which extent SLIC outlines coincide with actual object outlines. 


\subsection{Results}



(a)

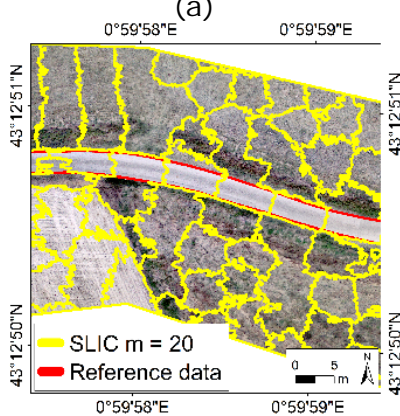

(d)

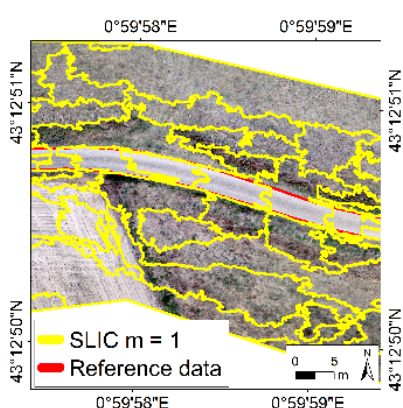

(b)



(e)



(C)

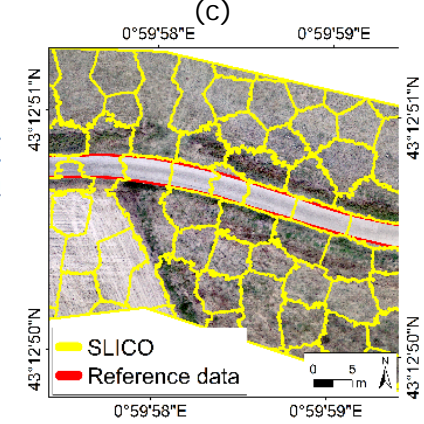

(f)

Figure 4.3. SLIC outlines derived for compactness parameters (a, b) $m=1$, (c, d) $\mathrm{m}=20$, and $(\mathbf{e}, \mathbf{f}) \mathrm{SLICO}$, where $\mathrm{m}$ is adaptively refined for each superpixel. The first row of images shows superpixels overlaid on the orthoimage of Amtsvenn, while the second row shows superpixels overlaid on the orthoimage of Toulouse, both for $k=10,000$.

Figure 4.3 shows that the regularity of the superpixel outlines can be enlarged by increasing $\mathrm{m}$ : the outlines of SLIC are more irregular for $m=1$ (Figure 4.3a/ b) than for $m=20$ (Figure 4.3c/ d). They run strictly along boundaries of spectral differences for $m=1$, while $m=20$ allows SLIC superpixels that are more homogeneous in shape, but less homogeneous in spectral content. This regularity in shape is increased even more, when using SLICO, for which $\mathrm{m}$ is automatically defined (Figure 4.3e/ f). The superpixels' outlines require further merging steps to delineate objects in the image as closed polygons. One approach might be to group SLIC superpixels of similar color. Another approach would be to merge SLIC outlines at locations, where another method with a higher detection quality, such as gPb contour detection, locates a boundary. 


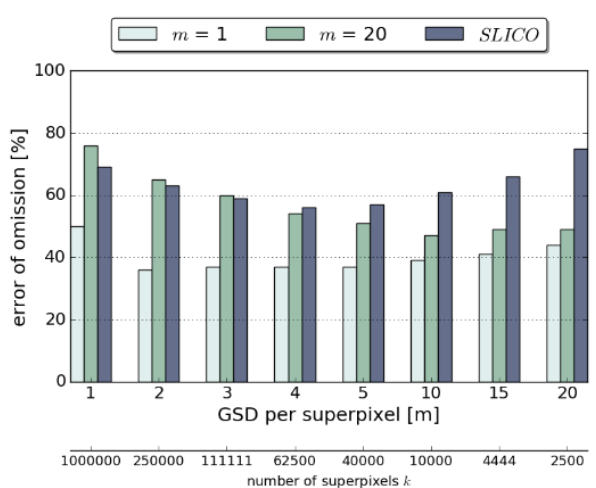

(a)

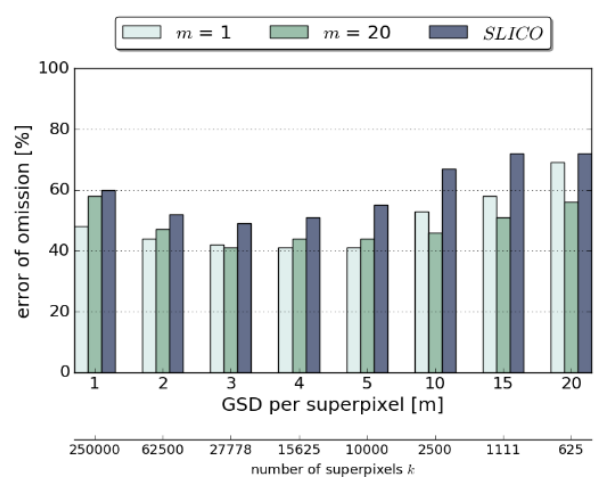

(b)

Figure 4.4. Errors of omission obtained for (a) Amtsvenn and (b) Toulouse. The number of superpixels k varies according to the extent covered per study area (Table 4.2).

Figure 4.4 shows the errors of omission for both study areas that range from $36 \%$ to $76 \%$. The error of omission is mostly lowest for $m=1$, regardless of the number of superpixels $k$. This observation holds true for data of Amtsvenn (Figure 4.4a) and Toulouse (Figure 4.4b). The allowed range for parameter $\mathrm{m}$ is $[0 ; \infty]$. The error of omission is mostly higher for SLICO compared to $\mathrm{m}=1$ and $\mathrm{m}=20$ across all investigated cases: due to the high shape regularity that SLICO enforces (Figure 4.4e/f), the superpixels become spectrally more heterogeneous and their outlines delineate the objects less accurately. The lowest errors of omission for both study areas are obtained for GSDs in the range [2; 5] $\mathrm{m}$ and amount to 36-37\% for Amtsvenn and to $41-$ $44 \%$ for Toulouse. For GSDs of $1 \mathrm{~m}$, the results of both study areas contain small areas for which the superpixel outlines appear regular-shaped, oriented in one direction and unaligned with object outlines. This effect might be caused by a memory problem due to the large number of generated superpixels. The predefined number of $\mathrm{k}$ and the obtained number of superpixels varies in median mean by $0.3 \%$. 


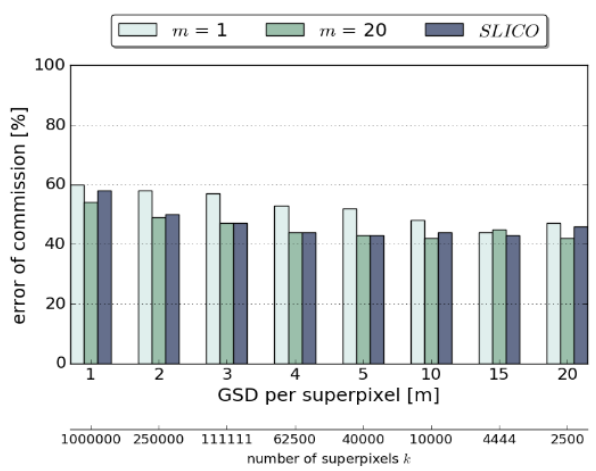

(a)

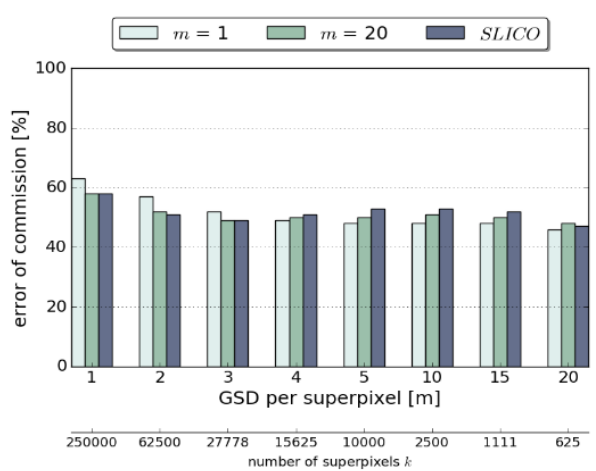

(b)

Figure 4.5. Errors of commission obtained for (a) Amtsvenn and (b) Toulouse. The number of superpixels $\mathrm{k}$ varies according to the extent covered per study area (Table 4.2).

Figure 4.5. shows the errors of commission for both study areas that range from $42 \%$ to $63 \%$. These numbers strongly depend on the chosen buffer size of $0.3 \mathrm{~m}$ around the reference data, in which FP pixels are counted. For a smaller buffer size, the errors of commission would be lower. This buffer size does not influence the error of omission, as this error considers boundary pixels in the reference map only. The errors of commission vary less per GSD and in terms of the SLIC parameters compared to the errors of omission. SLIC outlines need to be closed even when no object outline is available in the image. This hinders the effacement of the error of commission. The results indicate that this effect, i.e., the relative number of erroneously labeled 'outline' pixels, occurs equally across all investigated cases.

\subsection{Discussion}

The results indicate that SLIC superpixels delineate object outlines most accurately and completely using SLIC with a compactness parameter $\mathrm{m}=1$ for superpixels' GSDs in the range [2; 5 ] $\mathrm{m}$. Depending on the extent covered in one orthoimage, this results in a different number of superpixels defined as $k$ (Table 4.2). The regularity of object outlines to be delineated can be considered when deciding on $\mathrm{m}$ or SLICO: SLICO results in more regularshaped outlines and can provide more suitable results, when the object outlines are regular as well. When applying SLIC, the regularity of shape outlines can be slightly increased by increasing $\mathrm{m}$.

The results from [263], in which SLIC is applied to satellite imagery of 0.5 $0.6 \mathrm{~m}$ GSD are closest to those obtained in this study. Csillik suggests using an initial superpixel size of $10 \times 10$ pixels and 10 iterations for the clustering and refinement of the superpixels. The same number of iterations was used in this study. It is proposed as default by MathWorks. The superpixel size proposed by Csillik would correspond to superpixels of $0.5 \mathrm{~m}$ GSD for the data 
of this study ( $k=4,000,000$ for Amtsvenn; $k=1,000,000$ for Toulouse). As the error of omission increased for GSDs below $2 \mathrm{~m}$ and corresponding values of $k$, these superpixel sizes were not analyzed in this study. Furthermore, UAV data can be analyzed by considering 3D information in addition to the orthoimage. Future work will investigate the usability of SLIC on digital surface models (DSM) as proposed by Csillik. This could be done by applying $\mathrm{gPb}$ contour detection and SLIC superpixels on a DSM. This would allow identifying high gradients in high, which indicate objects such as fences or walls. Incorporating such information could help to localize missed outlines and to erase shadow outlines that are erroneously captured as object outlines (Figure 4.3a/ c/ e).

The accuracy assessment applied in this study is based on [258] and is similarly employed in numerous further studies $[228,255,257,299]$. It provides a comprehensive and widely used measure for positional accuracy. Disadvantages include its dependency on the applied buffer size and its sole focus on positional accuracy. For this study, it adequately measures to which extent SLIC outlines coincide with actual object outlines. More extensive accuracy assessment approaches suitable for the described application are listed in [44].

Furthermore, the manually delineated object outlines can contain errors. However, the applied buffer of $0.1 \mathrm{~m}$ partly smoothes inaccurately delineated outlines. Inaccuracies might be further reduced by averaging the manually delineated outlines of multiple human operators [94]. In general, manually drawn reference data are accepted to measure the degree to which an automated system outperforms a human operator [256].

Even for a workflow that accurately and completely delineates objects from UAV orthoimages, future work is required to determine the number of cadastral boundaries that are visible and can thus be extracted automatically. However, even a partial extraction of cadastral boundaries could improve the mapping procedure in terms of cost and time. Furthermore, an accurate and complete delineation of objects can be useful in further application fields such as topographical mapping, road tracking, or building extraction.

\subsection{Conclusion}

This study investigates automatic object delineation from optical UAV data. This supports multiple application fields such as recent endeavors in cadastral mapping, which aim to automatically delineate objects that demarcate cadastral boundaries from high-resolution optical sensor data. In this application field, a suitable workflow is assumed to consists of multiple feature extraction methods [44]. This study has investigated the potential of SLIC 
superpixels to delineate objects as part of such a workflow: SLIC was found to be applicable to UAV orthoimages and feasible to accurately delineate object outlines taking into account the high-resolution of $0.05 \mathrm{~m}$ provided by the UAV orthoimages.

However, the method generates a large number of outlines that do not demarcate object outlines. Future work will investigate the combination of SLIC with the contour detection method proposed in [240]. This contour detection method has shown to provide a comprehensive initial detection of candidate objects that could be verified and located exactly by integrating SLIC outlines. In addition, information from DSMs is intended to be incorporated along with the information from RGB orthoimages.

The goal is a tool for cadastral boundary delineation that is highly automatic, generic and adaptive to different scenarios. The tool will be most suitable for areas in which objects are clearly visible and coincide with cadastral boundaries. Once the design and implementation of such a tool is tested, its transferability to real-world scenarios will be investigated. This will be done in countries like Kenya, Rwanda and Ethiopia, where concepts like fit-for-purpose [6] and responsible land administration [259] are accepted or in place. 


\section{Interactive Boundary Delineation from UAV Data* $^{*}$}

\footnotetext{
* This chapter is based on:
}

300. Crommelinck, S.; Höfle, B.; Koeva, M.; Yang, M.Y.; Vosselman, G. In Interactive Boundary Delineation from UAV Data, ISPRS Annals of the Photogrammetry, Remote Sensing and Spatial Information Sciences, Riva del Garda, Italy, 04-07 J une, 2018; IV2, pp 81-88. 


\begin{abstract}
Unmanned aerial vehicles (UAV) are evolving as an alternative tool to acquire land tenure data. UAVs can capture geospatial data at high quality and resolution in a cost-effective, transparent, and flexible manner, from which visible land parcel boundaries, i.e., cadastral boundaries are delineable. This delineation is to no extent automated, even though physical objects automatically retrievable through image analysis methods mark a large portion of cadastral boundaries. This study proposes (i) a methodology that automatically extracts and processes candidate cadastral boundary features from UAV data, and (ii) a procedure for a subsequent interactive delineation. Part (i) consists of two state-of-the-art computer vision methods, namely gPb contour detection and SLIC superpixels, as well as a classification part assigning costs to each outline according to local boundary knowledge. Part (ii) allows a user-guided delineation by calculating least-cost paths along previously extracted and weighted lines. The approach is tested on visible road outlines in two UAV datasets from Germany. Results show that all roads can be delineated comprehensively. Compared to manual delineation, the number of clicks per $100 \mathrm{~m}$ is reduced by up to $86 \%$, while obtaining a similar localization quality. The approach shows promising results to reduce the effort of manual delineation that is currently employed for indirect (cadastral) surveying.
\end{abstract}




\subsection{Introduction}

Unmanned aerial vehicles (UAVs) are rapidly developing and increasingly applied in remote sensing, as they fill the gap between ground based sampling and airborne observations. Numerous application fields make use of the costeffective, flexible and rapid acquisition system delivering orthoimages, point clouds and digital surface models (DSMs) of high-resolution [14,39].

Recently, the use of UAVs in land administration is expanding $[12,41,241,301,302]$ : the high-resolution imagery is often used to visually detect and manually delineate cadastral boundaries. Such boundaries outline land parcels, for which additional information such as ownership and value are saved in a corresponding register [303]. The resulting cadastral map is considered crucial for a continuous and sustainable recording of land rights, as it allows the establishment of bureaucratic systems of fiscal and juridical nature and simplifies economic decision-making [7].

Worldwide, land rights of over $75 \%$ of the population are unrecognized, wherefore innovative, affordable, reliable, transparent, scalable and participatory tools for fit-for-purpose and responsible land administration are sought [6]. Automatically extracting visible cadastral boundaries from UAV data by providing a publicly available approach to edit and finalize those boundaries would meet this demand and improve current mapping procedures in terms of cost, time and accuracy [304].

This study describes advancements in developing a corresponding approach for UAV-based mapping of visible cadastral boundaries. Our study is based on the assumption that a large portion of cadastral boundaries is manifested through physical objects such as hedges, fences, stone walls, tree lines, roads, walkways or waterways. Those boundaries, visible in the RGB as well as the DSM data, offer the potential to be extracted in part automatically [42]. The extracted outlines require (legal) adjudication and incorporation of local knowledge from human operators in order to derive final cadastral boundaries.

In past work, a hypothetical generalized workflow for the automatic extraction of visible cadastral boundaries has been proposed [44]. The workflow was derived from 89 studies that extract physical objects related to those manifesting cadastral boundaries from high-resolution optical sensor data. The synthesized methodology consists of image segmentation, line extraction and contour generation (Figure 5.1). For image segmentation, globalized probability of boundary $(\mathrm{gPb})$ contour detection was found to be applicable for an initial detection of visible boundaries. However, the method does not enable the processing of large images. Therefore, the UAV data were reduced in resolution, which led to a reduced localization quality [240]. The localization 
quality at the locations of initially detected candidate boundaries is improved through the proceeding workflow component. For line extraction, simple linear iterative clustering (SLIC) superpixels applied to the full-resolution data were found to coincide largely with object boundaries in terms of completeness and correctness [261].

The aim of this study is to describe the final workflow component of contour generation: gPb contour detection and SLIC superpixels are combined with a random forest (RF) classifier and processed in a semi-automatic procedure that allows a subsequent delineation of visible boundaries. Overall, the study contributes to advancements in developing a methodology for UAV-based delineation of visible cadastral boundaries. The workflow uses RGB and DSM information and is designed for rural areas, in which physical objects such as roads are clearly visible and are anticipated to coincide with fixed cadastral boundaries.

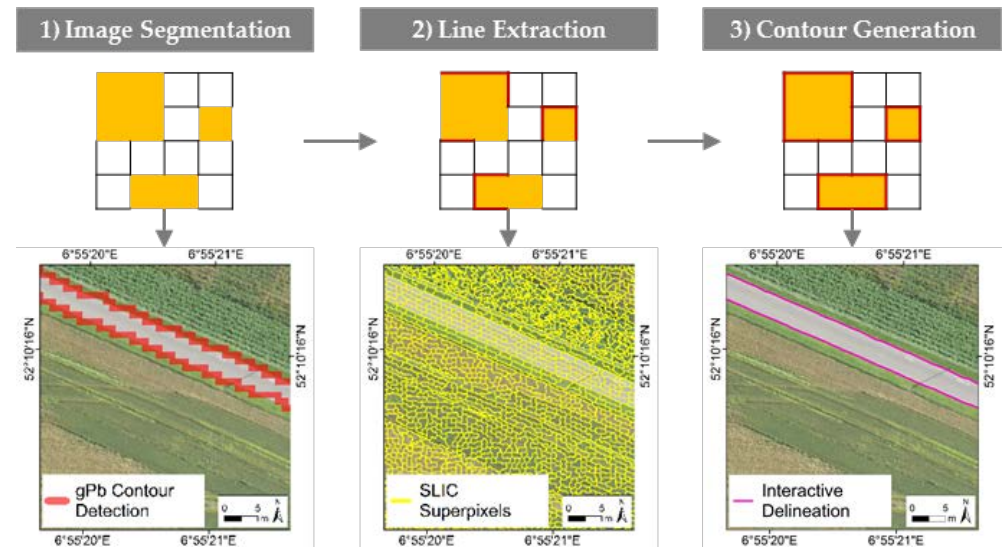

Figure 5.1. Sequence of a commonly applied workflow proposed in [44].The workflow aims to extract physical objects related to those manifesting cadastral boundaries from high-resolution optical sensor data. For the first and second component, state-of-the-art computer vision approaches have been evaluated separately and determined as efficient for UAV-based cadastral mapping $[240,261]$. The third component as well as the overall approach is described in this paper.

\subsection{Materials and Methods}

\subsubsection{UAV Data}

Two rural areas in Amtsvenn and Gerleve in Germany were selected for this study (Table 5.1, Figure 5.2). The data were captured with indirect georeferencing, i.e., Ground Control Points (GCPs) were distributed within the field and measured with a Global Navigation Satellite System (GNSS). RGB orthoimages as well as DSMs were generated with Pix4DMapper. 
Table 5.1. Specifications of UAV data.

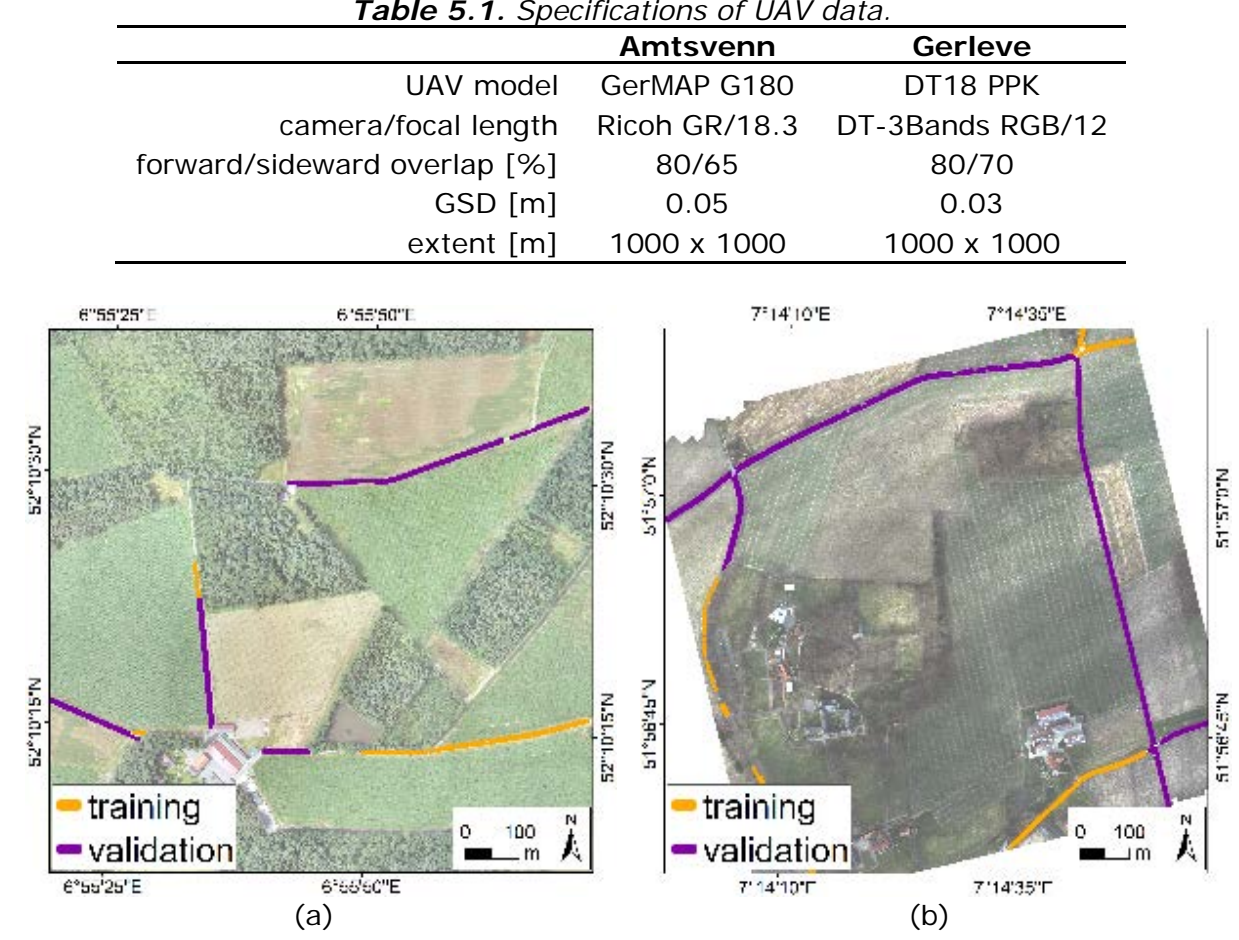

Figure 5.2. UAV data from (a) Amtsvenn and (b) Gerleve overlaid with SLIC lines used for training $(30 \%)$ and validation $(70 \%)$.

\subsubsection{Image Processing Workflow}

The image processing workflow is based on the one shown in Figure 5.1. Its three components are described in chapters 5.3.2.1-5.3.2.3. Corresponding source code, with test data and a step-by-step guide is publically available [305]. The interactive component is implemented in an open source GIS [306].

\subsubsection{Image Segmentation - gPb Contour Detection}

Contour detection refers to finding closed boundaries between objects or segments. Globalized probability of boundary $(\mathrm{gPb})$ contour detection refers to the approach visualized in Figure 5.3, explained in this section and based on [116]. This approach originates from computer vision and aims to find closed boundaries between objects or segments in an image. This is achieved through combining edge detection and hierarchical image segmentation, while integrating image information on texture, color and brightness on both a local and a global scale.

In a first step, oriented gradient operators for brightness, color and texture are calculated on two halves of differently scaled discs to obtain local image information. The cues are merged based on a logistic regression classifier 
resulting in a posterior probability of a boundary, i.e., an edge strength per pixel. The global image information is obtained through spectral clustering detecting the most salient edges only. This is done by examining a radius of pixels around a target pixel in terms of oriented gradient operators as for the local image information. The local and global information are combined through learning techniques and trained on natural images from the 'Berkeley Segmentation Dataset and Benchmark' [247]. By considering image information on different scales, relevant boundaries are verified, while irrelevant ones, e.g., in textured regions, are eliminated. This is referred to as global optimization in the following. In the second step, initial regions are formed from the oriented contour signal provided by a contour detector through oriented watershed transformation. Subsequently, a hierarchical segmentation is performed through weighting each boundary and their agglomerative clustering to create an ultrametric contour map (ucm) that defines the hierarchical segmentation.

The overall result consists of (i) a contour map, in which each pixel is assigned a probability of being a boundary pixel, and (ii) a binary boundary map containing closed contours, in which each pixel is labeled as 'boundary' or 'no boundary'. The approach has been shown to be applicable to UAV orthoimages for an initial localization of candidate object boundaries [240]. UAV orthoimages of extents larger than $1000 \times 1000$ pixels need to be reduced in resolution, due to the global optimization of the original implementation. The localization quality of initially detected candidate boundaries is improved through the following workflow components that use the full-resolution RGB and DSM data.



Figure 5.3. Workflow of globalized probability of boundary $(\mathrm{gPb})$ contour detection and hierarchical image segmentation resulting in a binary boundary map containing closed boundaries.

\subsubsection{Line Extraction - SLIC Superpixels}

Simple linear iterative clustering (SLIC) superpixels originate from computer vision and are introduced in [262]. Superpixels aim to group pixels into perceptually meaningful atomic regions and can therefore be located between pixel- and object-based approaches. The approach allows to compute image 
features for each superpixel rather than each pixel, which reduces subsequent processing tasks in complexity and computing time. Further, the boundaries of superpixels adhere well to object outlines in the image and can therefore be used to delineate objects [264].

When comparing state-of-the-art superpixel approaches, SLIC superpixels have outperformed comparable approaches in terms of speed, memory efficiency, compactness and correctness of outlines [263,266,267]. The approach, visualized in Figure 5.3, was introduced and extended by Achanta el al. $(2010,2012)$. SLIC considers image pixels in a 5D space, in terms of their $L^{*} a^{*} b$ values of the CIELAB color space and their $x$ and $y$ coordinates. Subsequently, the pixels are clustered based on an adapted k-means clustering. The clustering considers color similarity and spatial proximity. SLIC implementations are widely available. This study applies the GRASS implementation [279].

The approach has been shown to be applicable to UAV orthoimages of $0.05 \mathrm{~m}$ ground sample distance (GSD) [261]. Further, cadastral boundaries demarcated through physical objects often coincide with the outlines of SLIC superpixels.

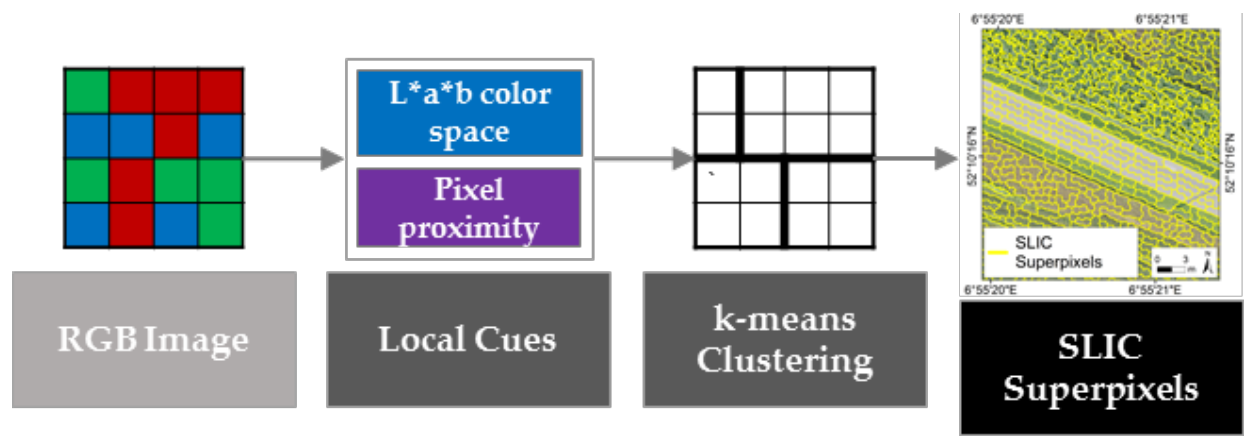

Figure 5.4. Workflow of simple linear iterative clustering (SLIC) resulting in agglomerated groups of pixels, i.e., superpixels, whose boundaries outline physical objects in the image.

\subsubsection{Contour Generation - Interactive Delineation}

Contour generation refers to generating a vectorized and topologically connected network of SLIC outlines from chapter 2.2.2 that surround candidate regions from chapter 2.2.1. This component combines the detection quality of $\mathrm{gPb}$ contour detection with the localization quality of SLIC superpixels. This is realized by seeking a subset of superpixels whose collective boundaries correspond to contours of physical objects in the image. 
Levinshtein et al. (2012) first reformulated the problem of finding contour closure to identifying subsets of superpixels that align with physical object contours. The authors combine features such as distance, strength, curvature and alignment to identify edges for image segmentation. These features are combined by learning the best generic weights for their combination on a computer vision benchmark dataset. This approach can be related to perceptual grouping in which local attributes in relation to each other are grouped to form a more informative attribute containing context information [307]. By iteratively grouping low-level image descriptions, a higher-level structure of higher informative value is obtained [308]. Perceptual grouping for contour closure is widely applied in computer vision [309,310], pattern recognition [308] as well as in remote sensing [178,194]. The criteria for perceptual grouping are mostly based on the classical Gestalt cues of proximity, continuity, similarity, closure, symmetry, common regions and connectedness that originate from Lowe's early work on perceptual grouping, in which a computational model for parallelism, collinearity, and proximity is introduced [311]. The attributes are mostly combined into a cost function that models the perceptual saliency of the resulting structure.

These ideas are transferable to this study: Wegner et al. (2015) extract road networks from aerial imagery and elevation data by applying superpixel-based image segmentation, classifying the segments with a RF classifier and searching for the Dijkstra least-cost path between segments with high likelihoods of being roads. Warnke and Bulatov (2017) extend this approach by optimizing the methodology in terms of feature selection. They investigate the training step by evaluating two classifiers and show that choosing features largely influences classification quality and that feature importance depends on the selected classifier. Similarly, García-Pedrero et al. (2017) use superpixels as minimum processing units, which is followed by a classification-based agglomerating of superpixels to obtain a final segmentation of agricultural fields from satellite imagery. All these approaches consider superpixels as segments, i.e., superpixels are agglomerated by comparing features per segment in relation to its adjacent neighbors [293,313,314], sometimes in combination with boundary information [315,316].

In this paper, the problem of finding adjacent superpixels belonging to one object is reformulated to finding parts of superpixel outlines that delineate one object: attributes are not calculated per superpixel, but per outline segment (Figure 5.4). They are created by splitting each superpixel outline, wherever outlines of three or more adjacent superpixels have a point in common. 19 attributes taking into account the full-resolution RGB and DSM, as well as the low-resolution $\mathrm{gPb}$ information are calculated per line (Table 5.2). Similar to the classical Gestalt cues, the attributes consider the SLIC lines themselves 
(i.e., their geometry) and their spatial context (i.e., their relation to gPb lines or to underlying RGB and DSM rasters).

Table 5.2. Features calculated per SLIC line segment.

\begin{aligned} & \hline Feature Description \\ & \hline length [m] $\begin{array}{l}\text { length per SLIC segment along the line } \\ \text { median of all ucm_rgb pixels within a 0.4m buffer around each } \\ \text { SLIC segment } \\ \text { median of all DSM laplacian filter values within a 0.4m buffer } \\ \text { lap_dsm } \\ \text { around each SLIC segment } \\ \text { distance between SLIC segment and gPb lines (overall shortest } \\ \text { distance) }\end{array} \\ &$ horizontal angle measured clockwise from north per SLIC \\ & azimuth [ ${ }^{\circ}$ segment \\ & ratio of distance between start and end point along SLIC \\ & segment (line length) and their direct Euclidean distance \\ & horizontal angle measured clockwise from north per gPb \\ & segment closest to a SLIC segment (aims \\ & to indicate line parallelism/collinearity) \end{aligned}

For training and validation, one attribute is added manually by labeling SLIC lines corresponding to reference object outlines as 'boundary' or 'no boundary', respectively. The data are divided into $30 \%$ for training and $70 \%$ for validation. The features shown in Table $\mathbf{5 . 2}$ together with the label 'boundary' or 'no boundary' are provided to the RF classifier to learn the combination of features leading to the class 'boundary' for the training data. The trained classifier then uses the features to predict for each line in the validation data a likelihood for each line for belonging to the class 'boundary'. This boundary likelihood b is transformed to a cost value $\mathrm{c}$ as shown in the following: 


$$
c[0 ; 1]=1-b
$$

$$
\begin{array}{ll}
\text { where } & c=\text { cost value per SLIC line } \\
& b=\text { boundary likelihood per SLIC line }
\end{array}
$$

This cost value $c$ in the range $[0 ; 1]$ is used to find the least-cost path between points indicated by a user. The Steiner least-cost path searches for the path along the SLIC lines having the lowest c, i.e., the highest likelihood for belonging to the class 'boundary'. The points represent start-, end, and optionally middle-points of a boundary to be delineated. Finally, the result is displayed to the user providing the options to accept, smooth, edit and/or save the line. Smoothing is done using the Douglas-Peucker line simplification. This interactive component is implemented as an open source QGIS plugin [306].

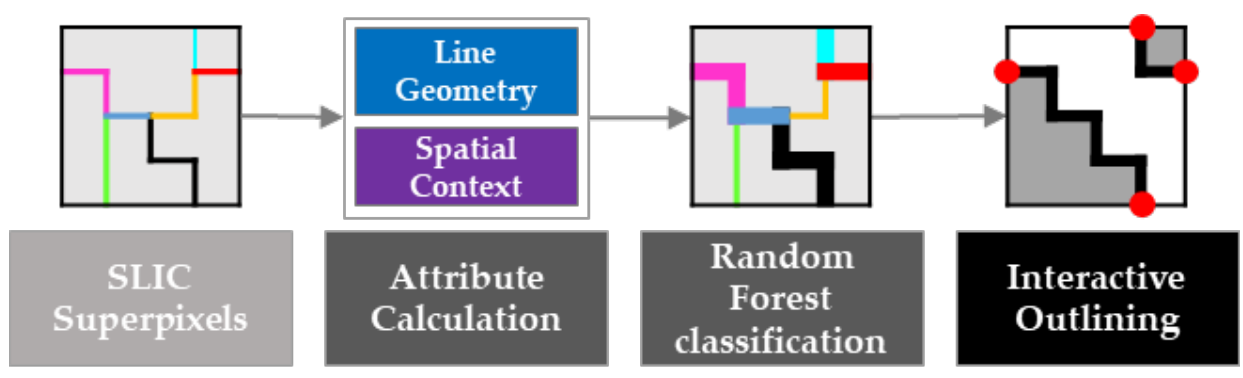

Figure 5.5. Workflow of interactive delineation: each superpixel outline is split, wherever outlines of three or more adjacent superpixels have a point in common (visualized by line color). Attributes are calculated per line. They are used by a RF classifier to predict boundary likelihoods (visualized by line thickness). User-selected nodes (red points) are connected along the lines of highest likelihoods.

\subsubsection{Accuracy Assessment}

The methodology is designed and implemented for rural areas, in which the number of visible cadastral boundaries is expected to be higher than in urban ones. As stated above, numerous physical objects can manifest cadastral boundaries. For accuracy assessment in a metric sense, an object was sought, whose outline is clearly delineable. Further, automating the delineation process saves most time for large parcels with long and curved outlines. Luo et al. [304] have shown that up to $49 \%$ of cadastral boundaries are demarcated by roads and conclude that deriving road outlines would therefore contribute significantly to generating cadastral boundaries. Consequently, roads are selected for accuracy assessment.

The approach is investigated in terms of the components shown in Figure 5.5. Since the first one, i.e., 'data pre-processing', has been evaluated in previous studies [240,261], the accuracy assessment focuses on 'classification' and the 'interactive outlining'. The accepted accuracy for cadastral boundary surveying depends on local requirements, regulations and the accuracy of the boundaries 
themselves. Recommendations from the IAAO [303] range from $0.3 \mathrm{~m}$ for urban areas to $2.4 \mathrm{~m}$ in rural areas for horizontal accuracy. They advise to use these measures judiciously and remain unclear whether this is a maximum for the accepted error or a standard deviation. According to Stock [317] landowners require a higher accuracy $(0.2 \mathrm{~m})$ than authorities $(0.5 \mathrm{~m})$ for rural boundaries. Details on how this accuracy is measured are not provided.

In this study, which is implemented in rural areas, the accepted accuracy is set to $0.2 \mathrm{~m}$ as maximum distance between delineation and reference data. Reference data are created through manually delineating visible outlines of roads. Only those visible outlines whose fuzziness did not exceed the accepted accuracy are delineated as reference data.

\subsubsection{Classification Performance}

How well the RF classifier assigns optimal costs to each SLIC line is crucial for the subsequent least-cost path generation. The performance is investigated by considering the feature importance obtained after applying the trained classifier on the validation dataset, as well as the confusion matrix and the derived correctness (Eq. 2) for different cost values c. Due to the analysis according to $c$, completeness is not considered: for larger $c$, more lines are detected, which makes the number of false negatives (FN) and thereby completeness not directly comparable across groups of different $c$.

$$
\begin{aligned}
& \text { correctness }[0 ; 100]=\frac{\mathrm{TP}}{\mathrm{TP}+\mathrm{FP}} \\
& \text { where TP }=\text { true positives } \\
& F P=\text { false positives }
\end{aligned}
$$

The detection quality (Figure 5.6a) determines, how comprehensively SLIC lines are detected by the RF classifier. This is done by calculating a buffer of radius $0.2 \mathrm{~m}$ around the reference lines. The buffer size is chosen in accordance to the pre-defined accepted accuracy. SLIC lines are buffered with the smallest radius possible of $0.05 \mathrm{~m}$ in accordance to the GSD of the UAV data. SLIC lines are grouped according to boundary likelihoods $b$, transformed to a cost value $c(2)$ in the range $[0 ; 1]$ at increments of 0.2 . Each group is overlaid with the buffered reference data to calculate a confusion matrix and a correctness.

The localization quality (Figure 5.6b) determines, if low c are assigned to segments located closer to the reference data. This is done by buffering the reference data with radii of $0.05,0.1,0.15$, and $0.2 \mathrm{~m}$. The previously buffered and grouped SLIC lines are reused. Each group is overlaid with the buffered reference data to generate a confusion matrix and to calculate the sum of TP pixels per buffer distance. 


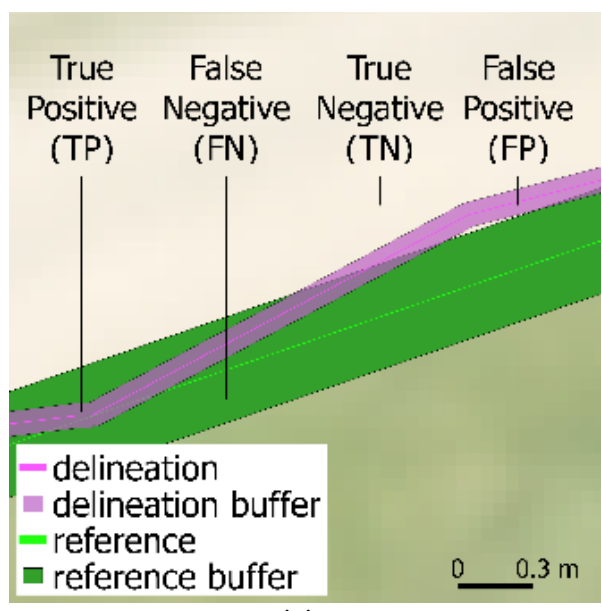

(a)

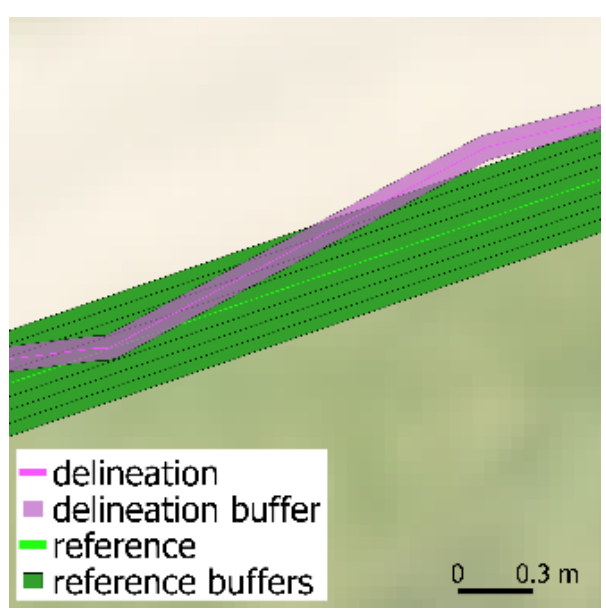

(b)

Figure 5.6. (a) Detection quality, for which delineation data are buffered with $0.05 \mathrm{~m}$ and reference data with $0.2 \mathrm{~m}$. Both are overlaid to calculate the number of pixels being TP, FN, TN or FP. (b) Localization quality, for which the reference data are buffered with 0.05-0.2 $\mathrm{m}$ and overlaid with the buffered delineation data to calculate the sum of TPs per buffer distance.

\subsubsection{Interactive Outlining Performance:}

If and to what extent the interactive delineation is superior to manual delineation is the focus of this section. This is done by defining a user scenario and delineating all visible road outlines once manually, once interactively. Metric accuracy measures are calculated for both datasets. The user scenario encompasses the guideline of using as few clicks as necessary to delineate all visible roads within the accepted accuracy of $0.2 \mathrm{~m}$. The metric accuracy measures consist of the calculation of the localization quality as described above and the average number of required clicks per $100 \mathrm{~m}$.

\subsection{Results}

The results reveal that the assignment of $\mathrm{c}$ works as desired: road outlines are comprehensively covered by SLIC lines of low $\mathrm{c}$ values and the correctness decreases for higher c (Table 5.3). Similarly, the localization quality mostly decreases for higher c, i.e., the classifier assigs low c values for a high percentage of lines close to the reference data (Figure 5.7). These values would vary when changing the buffer size or taking into account different lines for training.

The calculated feature importance for features shown in Table 5.2 reveals that higher-order features are often more valuable, i.e., a feature containing the gradient between green values right and left of the SLIC line (green_grad) is more important than a feature containing averaged green values underlying a 
SLIC line (I_gre_medi, r_gre_medi). DSM-related features have low importance (dsm_grad, lap_dsm, r_dsm_medi, I_dsm_medi), which can be increased by considering another physical object, whose outlines are stronger demarcated through height difference and by using relative height as a feature. gPb-related features (ucm_rgb, dist_to_gPb, azi_gPb) have a low importance, which might be caused by the low resolution of the gPb data. Tiling does not solve this problem since the global optimization requires image information on a global scale. However, gPb contours are still relevant as they are used to narrow down the area of investigation and thus reduce processing time. The results give an initial estimation of feature importance, but would require more data to analyzable in depth.

Table 5.3. Classification performance: detection quality for SLIC lines of different cost value c compared to reference data.

\begin{tabular}{|c|c|c|c|}
\hline & & Amtsvenn & Gerleve \\
\hline & SLIC line segments $(\mathrm{N})$ & 22,183 & 57,500 \\
\hline & SLIC line segments [m] & 37,063 & 72,333 \\
\hline \multirow{4}{*}{ 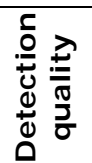 } & correctness $(c=0.0-0.19)[\%]$ & 86 & 93 \\
\hline & correctness $(c=0.2-0.39)[\%]$ & 90 & 96 \\
\hline & correctness $(c=0.4-0.59)[\%]$ & 78 & 88 \\
\hline & correctness $(c=0.6-0.79)[\%]$ & 61 & 70 \\
\hline
\end{tabular}

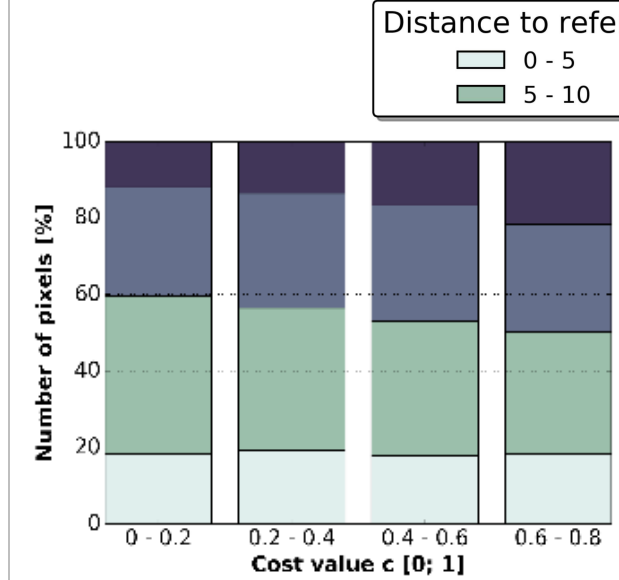

(a)

$\square-15$
$10-20$
15



(b)

Figure 5.7. Classification performance: localization quality for SLIC lines of different cost values c assigned through the RF classification for (a) Amtsvenn and (b) Gerleve.

The interactive outlining performance visualized in Figure 5.9 reveals that road outlines are successfully demarcated by low c values generated through RF classification (a). The interactive delineation visualized in (b) allows to select nodes (yellow) from a set of nodes (red), that are automatically connected (green) along the SLIC lines of least cost. The interactive delineation saves most clicks, when delineating long and curved roads as shown in (c), 
where the interactive delineation of a line of $274 \mathrm{~m}$ length requires two clicks only. For road parts covered by vegetation or those having narrow or fuzzy boundaries manual delineation is superior $(\mathrm{d})$. High gradients inside a road can cause the least-cost path to run along the middle of the road, which can be avoided by placing an additional node (yellow) along the road outline $(e, f)$. The least-cost path favors less segments of high costs over more segments of lower costs since the summated costs of the entire path are considered $(\mathrm{g})$. Created outlines can be smoothed out through the build-in line simplification that transforms the initial least-cost path (blue) to a simpler path (green) (h).

In general, all visible road outlines were delineated. For Amtsvenn $0 \%$ and for Gerleve $5 \%$ of lines required minor editing, in cases, where SLIC outlines do not run along the desired road outline (Figure 5.8d). The localization quality (Figure 5.9) visualizes the portion of delineated lines located at different distances to the reference data. Results show that for Amtsvenn almost $60 \%$ and for Gerleve almost $80 \%$ of boundaries delineated with the interactive approach are within $10 \mathrm{~cm}$ of the reference data. These results together with the decrease of required clicks, i.e., a reduction by $86 \%$ for Amtsvenn and 76\% for Gerleve (Table 5.4), and the lower zoom level required for delineation, shows that the interactive delineation is superior in terms of effort to delineate visible roads from UAV data.

Table 5.4. Interactive outlining performance: general statistics for the manual and the interactive delineation

\begin{tabular}{rcccc}
\hline & \multicolumn{2}{c}{ Amtsvenn } & \multicolumn{2}{c}{ Gerleve } \\
& manual & interactive & manual & interactive \\
\hline line segments [m] & 1,900 & 1,915 & 3,911 & 3,922 \\
avg. clicks per & $14.2(100 \%)$ & $2.3(86 \%)$ & $21.2(100 \%)$ & $4.5(76 \%)$ \\
$100 m(N)$ & $10.2(10 \%)$ &
\end{tabular}




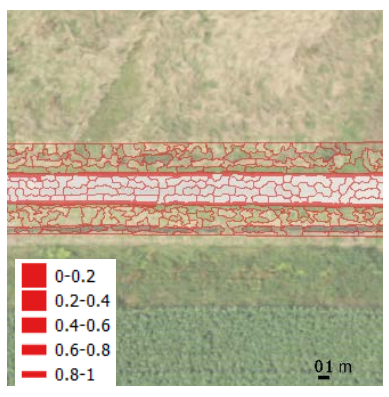

(a)

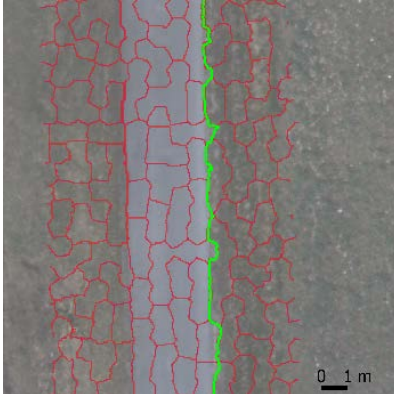

(d)

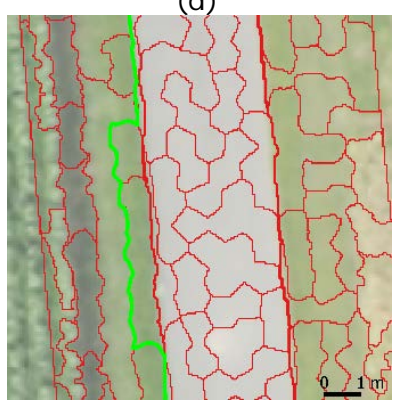

(g)



(b)

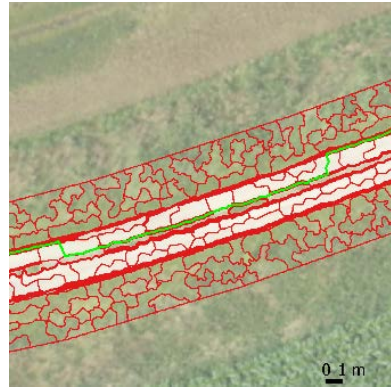

(e)

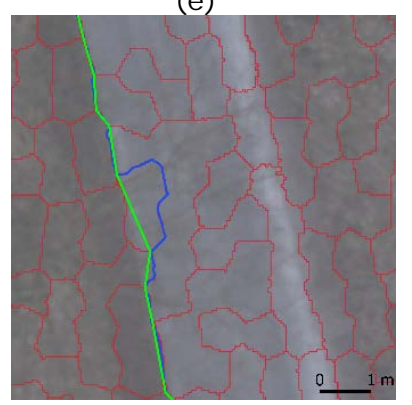

(h)

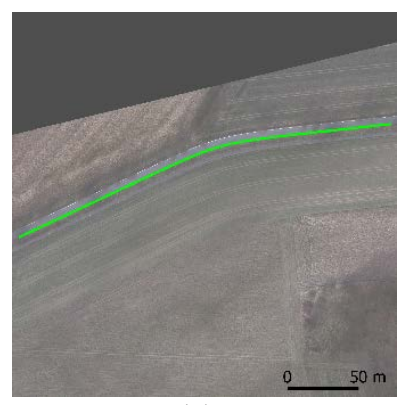

(c)



(f)

Figure 5.8. Examples of the interactive delineation (green) along SLIC lines (red). The thicker a SLIC line, the lower c. 


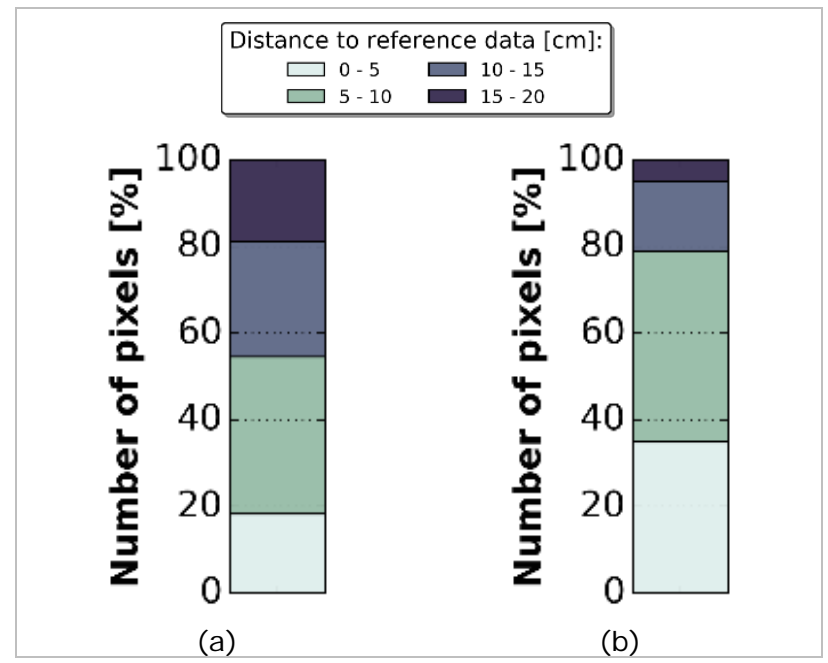

Figure 5.9. Interactive outlining performance: localization quality for delineation for (a) Amtsvenn and (b) Gerleve. Both the reference and the interactively delineated data consists of lines that are rasterized to quantify the localization quality.

\subsection{Discussion}

In general, the methodology could improve current indirect mapping procedures by making them more reproducible and efficient. However, a certain skill level of the surveyors in geodata processing is required as well as the presence of visible cadastral boundaries. With cadastral boundaries being a human construct, certain boundaries are not automatically detectable, wherefore semi-automatic approaches are required [304].

Limitations of the accuracy assessment are as follows: labelled training data doesn't always coincide exactly with the reference data, as SLIC outlines do not perfectly match the manually delineated road outlines. Furthermore, some roads have fuzzy outlines, wherefore a certain outline is selected within the accepted accuracy for both the manual and the interactive delineation. Furthermore, manual image interpretation is prone to produce ambiguous results due to interpreters generalizing differently. These uncertainties propagate through the accuracy measures and would increase when considering physical objects of fuzzier outlines [313,318]. Further, the percentage of roads demarcating cadastral boundaries, which according to Luo et al. (2017) amounts up to $49 \%$ might be lower in certain cases. Further work should be conducted considering various objects in relation to real cadastral reference data.

Future work could focus on identifying optimal features for classification $[312,319]$. The optimal selection of training data could be supported by active learning strategies. Another focus would be to extent the approach to different 
physical objects, datasets and scenarios by developing a classifier transferable across scenes. However, even manually labeling $30 \%$ of the data before being able to apply the interactive delineation as done in this study, would still be superior in terms of effort than delineating $100 \%$ manually. Existing cadastral data might be used to automatically generate training data. The transferability to data from aerial or satellite platforms could be considered to determine the degree to which high-resolution UAV data containing detailed 3D information is beneficial or required for indirect cadastral surveying. Further, the least-cost paths generation can be improved by scaling the line costs with their length to avoid the path favoring few segments of high cost over many segments of low costs (Figure $\mathbf{5 . 8 g}$ ). In addition, sharp edges in the generated least-cost path can be penalized to reduce outlier occurrence, as done in snake approaches.

\subsection{Conclusion}

This study contributes to developing a methodology for UAV-based delineation of visible cadastral boundaries. This is done by proposing a methodology that partially automates and simplifies the delineation of outlines of physical objects such as roads demarcating cadastral boundaries. Previous work has focused on automatically extracting RGB image information for that methodology. In this paper, the methodology is extended by a classification and an interactive outlining part applied to RGB and DSM data. Furthermore, this study proposes a methodology to automate cadastral mapping covering all required steps after obtaining UAV data to generating candidate cadastral boundary lines.

The reformulated problem of delineating physical objects from image data to combining line feature information with RF classification presented in this study, could be beneficial for different delineation applications. The aim of this study is to apply the suggested approach for cadastral mapping. In this field, the approach has shown promising results to reduce the effort of current indirect surveying approach based on manual delineation. Highest savings are obtained for long and curved outlines. Future work will focus on the methodology's transferability to real-world cadastral mapping scenarios. 


\section{Validating and Improving Automated Feature Extraction for UAV-based Cadastral Mapping*}

\footnotetext{
* This chapter is based on:

320. Crommelinck, S.; Koeva, M.; Yang, M.Y.; Vosselman, G. Robust Object Extraction from Remote Sensing Data. arXiv: 1904.12586 2019.
} 


\section{Abstract}

The extraction of object outlines has been a research topic during the last decades. Despite advances in photogrammetry, remote sensing, and computer vision, this task remains challenging due to object and data complexity. This challenge amplifies for cadastral boundaries demarcated by various objects with different characteristics. Meanwhile, with land rights being sensitive, knowledge-, and context-dependent, the scope of automation in cadastral mapping seems limited. We addressed these circumstances by proposing an approach that couples a machine-based automatic feature extraction with a delineator-based interactive delineation. In this chapter, we validate and improve this indirect surveying approach by comparing it to manual delineation: (i) the accuracy analysis questions how correct and complete visible objects can be delineated with the approach compared to manual delineation, (ii) the operational analysis questions when and why the approach works better or worse compared to manual delineation, and (iii) the feedback analysis investigates the approach based on feedback from surveying stakeholders. As a result, we gain an understanding and an overview of current bottlenecks, which enables us to identify and prioritize areas for further development. 


\subsection{Introduction}

This chapter introduces improvements in automated feature extraction for UAV-based cadastral mapping compared to [300], describes the approach's current functioning, demonstrates results obtained in different scenarios, and synthesizes user feedback. The areas of investigation are a peri-urban site in Rwanda, as well as, a rural and a peri-urban site in Kenya. The aim is to gain an understanding and an overview of current bottlenecks, in order to identify and prioritize areas for further development. This is done by analyzing the boundary mapping approach compared to manual delineation as follows:

(i) The accuracy analysis questions how correct and complete visible objects can be delineated with the approach when considering manual delineation as ground truth. This is done by conducting a line-based accuracy assessment of quantitative completeness and spatial correctness for precisely extractable object outlines of buildings and cadastral boundaries delineated once with the approach, once manually by local experts.

(ii) The operational analysis questions when and why the approach is more efficient compared to manual delineation. This is done taking into account observations from the delineation for the accuracy analysis and an additional delineation to provide an overview of successful and challenging delineation cases.

(iii) The feedback analysis investigates the approach based on feedback from stakeholders having a background or an affiliation with surveying. This is done by analyzing the approach in terms of strengths, weaknesses, opportunities, and threats (SWOT). Feedback is derived from three oneday workshops for land administration stakeholders from local government institutions, NGOs, private companies, and national government institutions.

\subsection{Materials and Methods}

\subsubsection{UAV data}

UAV data from four different sites in Rwanda and Kenya are used for this chapter. The data were captured with indirect georeferencing, i.e., Ground Control Points (GCPs) were distributed within the field and measured with a Global Navigation Satellite System (GNSS). RGB orthomosaics and DSMs were generated with Pix4DMapper. Specifications of the data capture are shown in Table 6.1. The UAV data are visualized in Figure 6.1-3. 
Validating and Improving Automated Feature Extraction

Table 6.1. Specifications of UAV data.

\begin{tabular}{|c|c|c|c|c|c|c|}
\hline Location & $\begin{array}{c}\text { UAV Model, } \\
\text { Brand }\end{array}$ & $\begin{array}{l}\text { UAV } \\
\text { Type }\end{array}$ & $\begin{array}{c}\text { UAV } \\
\text { Sensor }\end{array}$ & $\begin{array}{c}\text { Capture } \\
\text { Date }\end{array}$ & $\begin{array}{l}\text { Area } \\
\text { [ha] }\end{array}$ & $\begin{array}{l}\text { GSD } \\
{[\mathrm{cm}]}\end{array}$ \\
\hline $\begin{array}{l}\text { Muhoza, } \\
\text { Rwanda }\end{array}$ & $\begin{array}{c}\text { FireFLY6, } \\
\text { BirdsEyeView }\end{array}$ & Hybrid & $\begin{array}{l}\text { SONY } \\
\text { A6000 }\end{array}$ & $\begin{array}{l}\text { Jan. } \\
2018\end{array}$ & 94 & 5 \\
\hline $\begin{array}{l}\text { Mukingo, } \\
\text { Rwanda }\end{array}$ & $\begin{array}{c}\text { Inspire 2, } \\
\text { DJI }\end{array}$ & $\begin{array}{l}\text { Rotary- } \\
\text { wing }\end{array}$ & $\begin{array}{c}\text { Zenmuse } \\
\text { X5S }\end{array}$ & $\begin{array}{l}\text { Jan. } \\
2018\end{array}$ & 50 & 5 \\
\hline $\begin{array}{l}\text { Kajiado, } \\
\text { Kenya }\end{array}$ & $\begin{array}{c}\text { Phantom 4, } \\
\text { DJI }\end{array}$ & $\begin{array}{l}\text { Rotary- } \\
\text { wing }\end{array}$ & FC330 & $\begin{array}{l}\text { Sep. } \\
2018\end{array}$ & 830 & 5.8 \\
\hline $\begin{array}{l}\text { Mailua, } \\
\text { Kenya }\end{array}$ & $\begin{array}{c}\text { DT18, } \\
\text { DelairTech }\end{array}$ & $\begin{array}{l}\text { Fixed- } \\
\text { wing }\end{array}$ & $\begin{array}{l}\text { DT18 } 3 \\
\text { bands }\end{array}$ & $\begin{array}{l}\text { March } \\
2018\end{array}$ & 330 & 5.7 \\
\hline
\end{tabular}

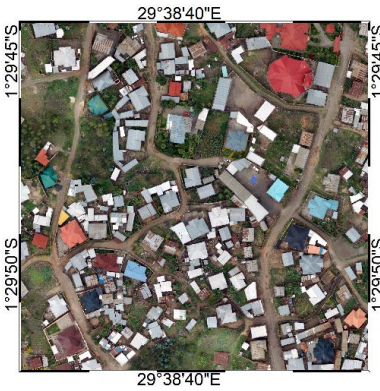

(a)



(d)

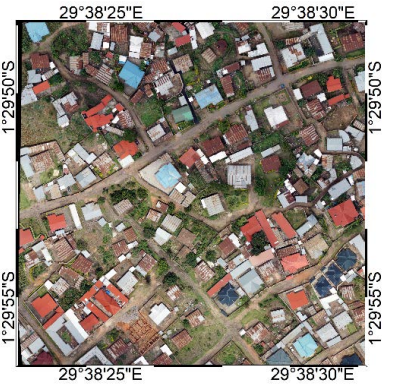

(b)

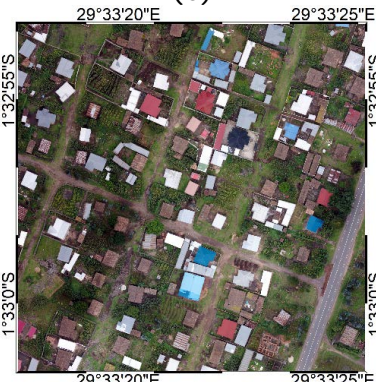

(e)

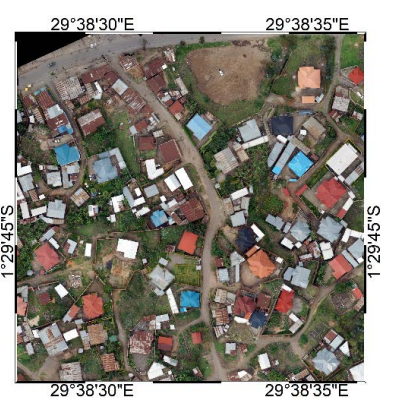

(c)



(f)

Figure 6.1. UAV data tiles of $250 \times 250 \mathrm{~m}$ and a $5 \mathrm{~cm}$ GSD of Muhoza (a-c) and Mukingo (d-f), Rwanda. 


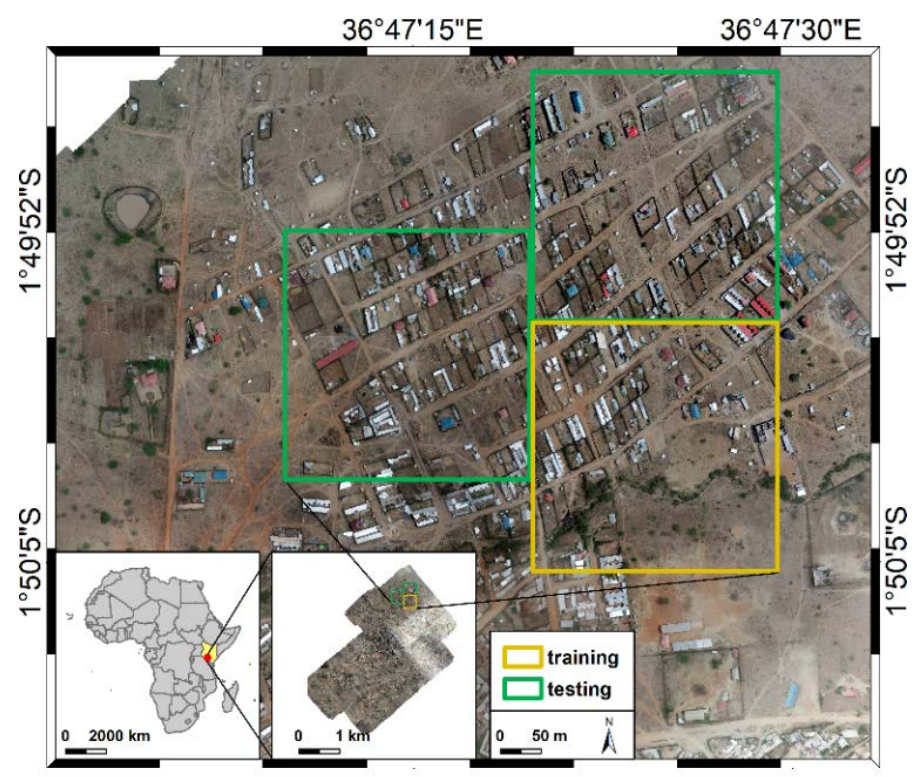

Figure 6.2. UAV data tiles of $250 \times 250 \mathrm{~m}$ and a $6 \mathrm{~cm}$ GSD of Kajiado, Kenya.

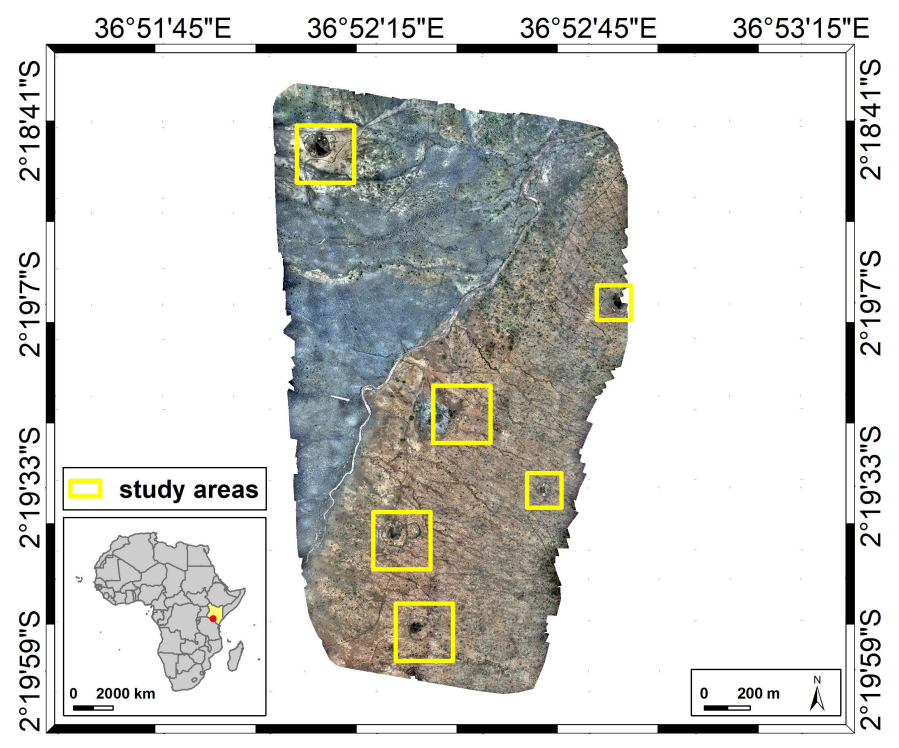

Figure 6.3. UAV data tiles of $250 \times 250 \mathrm{~m}$ and $150 \times 150 \mathrm{~m}$ and a $6 \mathrm{~cm}$ GSD of Mailua, Kenya. 


\subsubsection{Boundary Delineation Approach}

The delineation approach is based on [300]. It supports the delineation of boundaries by automatically retrieving information from RGB and DSM data that is then used to guide an interactive delineation. The approach consists of three parts: (i) image segmentation, (ii) boundary classification, and (iii) interactive delineation (Figure 6.4). Each part is addressed in the following describing its current functioning and changes compared to what has been proposed in the previous chapter and in [300]. The source code is publically available [305].

The source code has been optimized in terms of performance, effectivity, consistency, and clearness as explained in the following. This aimed to make the code accessible in a server environment or a Docker container. Changes include the replacement of former QGIS or GRASS functions by OGR or GDAL functions, which are accessible without a QGIS installation; the storing of the code configurations in JSON structure; the adding of logging and error handing, the replacement of temporarily stored shapefiles as GeoJ SON structures; and the conversion from Python 2.7 to Python 3, from QGIS 2 to QGIS 3, and from PyQt 4 to PyQt 5.

(i) I mage segmentation delivers closed contours capturing the outlines of visible objects in the image. Multiresolution Combinatorial Grouping (MCG) [117] has shown to be applicable to high-resolution UAV data and to deliver accurate closed contours of visible objects [320].

Changes: The workflow described in the previous chapter proposes to use Globalized Probability of Boundary (gPb) [116] and Simple Linear Iterative Clustering (SLIC) superpixels [114]. We now propose to use an extended version of $\mathrm{gPb}$ developed by the same authors: Multiresolution Combinatorial Grouping (MCG) [117]. This allows combining the previous steps of image segmentation and line extraction into one method while increasing spatial accuracy compared to using gPb and decreasing oversegmentation compared to using SLIC.

(ii) Boundary classification requires labeling the contours from (i) into 'boundary' and 'not boundary' to generate training data. A set of features is calculated per line capturing its geometry (i.e., length, number of vertices, azimuth, sinuosity) and its spatial context (i.e., gradients of RGB and DSM underlying the line).

(iii) Table 6.2 shows all features being calculated, of which the first two are not used for the classification. These features together with the labels are used to train a Random Forest (RF) classifier [321]. The trained classifier predicts boundary likelihoods for unseen testing data for which the same features have been calculated, as indicated with training and testing in 
Figure 6.4. An open-source RF implementation [322] is used for the classification.

Changes: The boundary classification proposed in the previous chapter aimed to generate a boundary likelihood combining information from $\mathrm{gPb}$ and SLIC. Since this combination is omitted, the features ucm_rgb and azi_gPb previously calculated per SLIC line to describe its geometrical relation to the closest $\mathrm{gPb}$ line are omitted as well. From an analysis of feature importance and a comparison with the state-of-the-art on feature selection, it appeared redundant to calculate similar features such as r_gre_medi, I_gre_medi, and green_grad. Therefore, we omitted calculating the redundant features $r$ _dsm_medi, I_dsm_medi, $r$ red_medi, I_red_medi, r_gre_medi, I_gre_medi, r_blu_medi, I_blu_medi, and kept the higher-order features dsm_grad, lap_dsm, r_dsm_medi, I_dsm_medi. For the same reason, we omitted calculating lap_dsm. We added vertices to account for geometrical line characteristics that were previously not captured. Optimizing features decreased the complexity and thus processing time of the boundary classification step. Table 6.3 shows all omitted features.

(iv) Interactive delineation allows a user to start the actual delineation: the RGB orthomosaic is displayed to the user, who is asked to select nodes to be connected to a boundary. A least-cost-path algorithm searches for the lines from (i) that connect the user-selected nodes taking into account the boundary likelihood from (ii). The line is suggested to the user with the options to edit, save or delete. We implemented (iii) as publically available plugin [306] for the open-source geographic information system QGIS [296].

Changes: The changes relate to interface design, coding quality, and added functionalities. A survey study [323] was conducted to identify and prioritize points for improvement of the interactive delineation. This was done by asking 13 volunteers with a geospatial background to delineate visible boundaries with the developed QGIS plugin. As main reasons for the plugin's partial inability to delineate cadastral boundaries, the following were identified: (i) land parcel boundaries are not visible, fuzzy, or irregular, (ii) plugin implementation errors, e.g., not-working of automatic renaming of layers or inability to connect large number of nodes, and (iii) QGIS software instability. (ii) and (iii) have been addressed by refactoring and restructuring the source code to be compliant with the recently released QGIS3 version. Before, the interactive delineation was implemented in QGIS2. The participants listed the following recommendations to improve the plugin: (i) separating the tasks of interactive delineation and manual editing more comprehensively in the GUI, (ii) docking the plugin to the QGIS toolbar to improve usability, (iii) decreasing the computing time for the creation of node and network 
files, which took up to 10 minutes, (iv) decreasing the number of proposed nodes to select from, (v) allowing to simplify created lines more comprehensively, (vi) allowing to connect a higher number of nodes, (vii) automatically enabling snapping in QGIS when plugin is opened, (viii) automatically keeping the last node of the previously created line selected, and (ix) including further topological checks of a created line besides its sinuosity. (i) Has been addressed by making a visual distinction between the interactive and the manual delineation in the GUI (Figure 6.4c). (ii) Has been implemented. (iii) Has been addressed by refactoring and restructuring of the source code; the calculation now takes a few seconds. (iv) Has been addressed by using MCG instead of SLIC lines for node creation. MCG over-segments less than SLIC, wherefore fewer nodes are created. ( $v$ ) Has been addressed by implementing a stronger DouglasPeucker line simplification. (vi) Has been addressed by refactoring and restructuring the source code for QGIS 3, allowing the connection of multiple nodes. (vii) Has been implemented. (viii) Has not been implemented, as this functionality would add complexity to the interactive delineation process and represents a use case that is not present in most delineation cases. (ix) The previous version displayed the line sinuosity as probable line usability in a traffic-light-fashion. The current implementation does not make use of line sinuosity any more, but uses the boundary likelihood instead: the line thickness indicates its boundary likelihood and thus gives a more comprehensive impression on its usefulness compared to the previously used sinuosity.

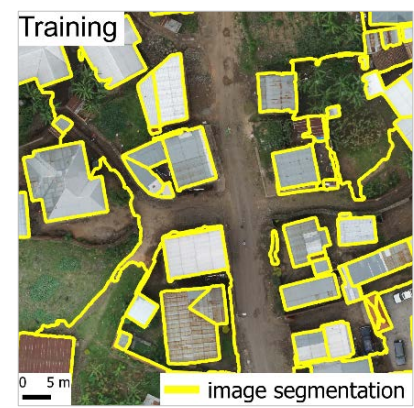

(a)

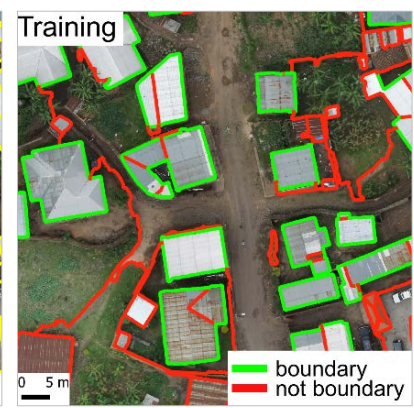

(b)

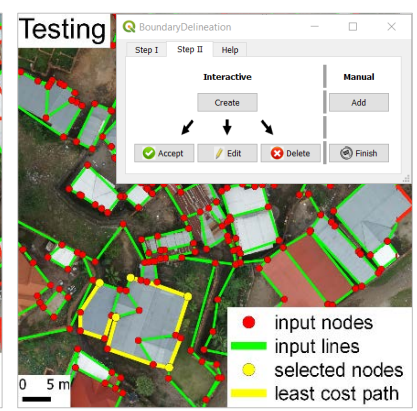

(c)

Figure 6.4. Boundary mapping approach: (a) MCG image segmentation. (b) Boundary classification that requires line labeling into 'boundary' and 'not boundary' for training. The labeled lines are used together with line-based features to train a Random Forest classifier that generates boundary likelihoods for testing. (c) Interactive delineation guided by a QGIS plugin that creates a least-cost-path between user-selected nodes along simplified lines from (a) with highest boundary likelihoods generated in (b). 
Table 6.2. Features calculated per line to be used by the Random Forest (RF) classifier for boundary classification.

\begin{tabular}{|c|c|c|}
\hline & Feature & Description \\
\hline \multirow{10}{*}{ 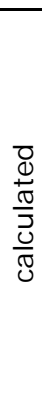 } & ID & Unique number per line \\
\hline & boundary & Boundary label or likelihood in range $[0 ; 1]$ \\
\hline & vertices & Number of vertices per line \\
\hline & length $[\mathrm{m}]$ & Length per line \\
\hline & azimuth $\left[{ }^{\circ}\right]$ & Bearing in degrees between start and end of each line \\
\hline & sinuosity & $\begin{array}{l}\text { Total line length divided by the shortest distance between } \\
\text { start and end of each line }\end{array}$ \\
\hline & red_grad & $\begin{array}{l}\text { Abs. difference between median of all red values lying within } \\
\text { a } 0.4 \mathrm{~m} \text { buffer right and left of each line }\end{array}$ \\
\hline & green_grad & Same as red_grad for green of RGB \\
\hline & blue_grad & Same as red_grad for blue of RGB \\
\hline & dsm grad & Same as red grad for DSM \\
\hline
\end{tabular}

Table 6.3. Omitted features calculated in previous version of approach [300].

\begin{tabular}{|c|c|c|}
\hline \multirow{12}{*}{ 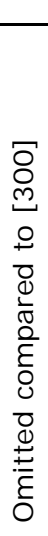 } & Feature & Description \\
\hline & ucm_rgb & median of all ucm_rgb pixels within a $0.4 \mathrm{~m}$ buffer around \\
\hline & lap_dsm & median of all DSM Laplacian filter values within a $0.4 \mathrm{~m}$ buffer \\
\hline & & around each SLIC segment \\
\hline & dist_to_gPb $[\mathrm{m}]$ & $\begin{array}{l}\text { distance between SLIC segment and gPb lines (overall } \\
\text { shortest distance) }\end{array}$ \\
\hline & azi_gPb $\left[^{\circ}\right]$ & horizontal angle measured clockwise from north per $\mathrm{gPb}$ \\
\hline & & $\begin{array}{l}\text { segment closest to a SLIC segment (aims to indicate line } \\
\text { parallelism/collinearity) }\end{array}$ \\
\hline & r_dsm_medi & $\begin{array}{l}\text { median of all DSM values lying within a } 0.2 \mathrm{~m} \text { buffer right of } \\
\text { each SLIC segment. }\end{array}$ \\
\hline & I_dsm_medi & Same as r_dsm_medi for left buffer of DSM \\
\hline & $\bar{r} \_$red_-medi & edi for right buffer of red of RGB \\
\hline & I_red_medi & Same as r_dsm_medi for left buffer of red of RGB \\
\hline & r_gre_medi & Same as r_dsm_medi for right buffer of green of RGB \\
\hline & I_gre_ & Same as $r_{-}$dsm_medi for left buffer of green of RGB \\
\hline & r_blu_medi & Same as r_dsm_medi for right buffer of blue of RGB \\
\hline & I blu medi & Same as $r$ dsm medi for left buffer of blue of RGB \\
\hline
\end{tabular}

\subsubsection{Accuracy Analysis}

The accuracy analysis questions how correct and complete visible objects can be delineated with the approach when considering manual delineation as ground truth. This is done by conducting a line-based accuracy assessment of (i) quantitative completeness, and (ii) spatial correctness for the delineation of buildings and cadastral boundaries. They are delineated based on UAV imagery once with the approach, once manually based on local knowledge, and compared thereafter. For buildings, manually delineated outlines of undamaged buildings having an area of $>25 \mathrm{~m}^{2}$ are considered. For cadastral boundaries, manually delineated parcel outlines are used.

(i) Quantitative Completeness investigates the percentage of objects that can be entirely delineated by the approach. After the interactive delineation, the number of objects, i.e., buildings or parcels, that are either 
directly acceptable without further editing, require editing, or require manual delineation are summated.

(ii) Spatial Correctness investigates to what extent successfully delineated objects coincide with the reference data in a spatial sense. This is done by first buffering the reference data. The buffer size should be chosen in accordance with the required accuracy of the object delineation. For cadastral mapping, the statutorily requested accuracy depends on the needs and nature of the area being surveyed. The measuring approach and the accepted result can vary $[303,324]$. Subsequently, the percentage of the delineation line lying inside and outside of the reference buffer is calculated. This can be done either vector- or raster-based. Inaccuracies in the reference data are expected to be much lower than the accuracy needed for cadastral boundary mapping.

For a raster-based approach, as done in this chapter, the delineation lines are rasterized to the $5 \mathrm{~cm}$ GSD of the input data. Only those delineation lines from (i) that were directly acceptable without further editing are used. The reference lines are buffered, rasterized and overlaid with the reference data (Figure 6.5). The overlay allows labeling pixels as TP, where the delineation line falls inside the reference buffer and as FP, where the delineation line falls outside the reference buffer. The sum of pixels with the same label is summarized in a confusion matrix. From the confusion matrix, the error of commission (Figure 6.5a) and the correctness (Figure $\mathbf{6 . 5 b}$ ) are calculated in the range [0; 100]. The error of commission captures the percentage of pixels falling outside of the reference buffer, the correctness those that fall inside of it. The buffer size should be chosen in accordance with locally accepted accuracies for cadastral boundary mapping. For this chapter the buffer size is set to a radius of $30 \mathrm{~cm}$ as justified in the following.

The accuracy measures are based on [325], described as commonly reported measures in more recent publications [72,224], and similarly used to evaluate the results of an ISPRS benchmark dataset [220]. We implemented the described procedure for line-based accuracy assessment consisting of buffering, rasterizing, overlaying, calculating and plotting the confusion matrix, in a publically available plugin (Figure 6.5c) [326] for QGIS [296]. 


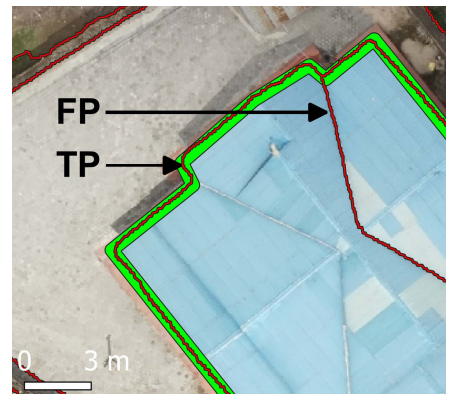

error of commission $[\%]=\frac{\mathrm{FP}}{\mathrm{FP}+\mathrm{TP}} * 100$

correctness $[\%]=\frac{\mathrm{TP}}{\mathrm{FP}+\mathrm{TP}} * 100=1-$ error of commission

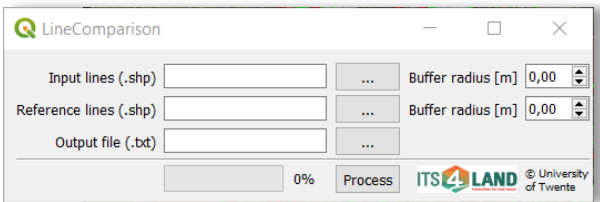

Figure 6.5. Spatial correctness based on overlaying the buffered delineation and reference data to compute pixels being True Positive (TP) or False Positive (FP). These pixels are then summated to calculate (a) the error commission and (b) the correctness. (c) We implemented the described procedure for line-based accuracy assessment as a new 'LineComparison' QGIS plugin.

In Rwanda, no legal planimetric accuracy standard has been defined. Cadastral records originate mostly from a cadastral mapping effort initiated in the 2000s. This mapping was based on general boundaries as stated in the Land Law. This mapping effort resulted from land reforms demanding a nationwide adjudication, surveying, and recording program, during which 12 million parcels were mapped over a period of four years in a participatory mapping approach. Locals were trained for a few days as para-surveyors to draw boundaries on printed satellite or aerial images that were afterwards digitized in the office. Speed and costs were prioritized over accuracy. After completion of the project, attendance has now shifted to maintenance and updating: the cadastral records are supposed to be continuously updated and replaced by fixed boundaries using Differential Global Positioning Systems (DGPS). This is currently realized with a focus on urban areas, for which coarse network systems are increasingly being established. During this updating process in land registration, measuring points are allowed to lie within a maximum of $25 \mathrm{~cm}$ radius.

For the Rwandan study areas of this chapter, the official cadastral reference data have been delineated in the described participatory mapping approach on aerial imagery of $25 \mathrm{~cm}$ GSD. This cadastral data are thus outdated and of low spatial accuracy. Two Rwandan surveyors with delineation expertise in surveying generated new cadastral reference data for the accuracy analysis of this chapter. They applied indirect surveying: parcel boundaries were delineated on the UAV orthomosaics based on local knowledge of boundary locations and characteristics, and in comparison with the original cadastral data.

In Kenya, for many years after independence in 1963, no clearly defined national land policy had been defined. This has resulted in complex and fragmented land management and administration systems that co-exist 
without being compatible. Different laws, regulations, and requirements have resulted in cadastral maps using different coordinate systems, scales and surveying methods. Fixed and general boundaries are applied both for parcel identification. Partly overlapping boundaries often cause land conflicts. Despite the recommendation in the national land policy from 2009 to establish a digital land information infrastructure as part of the National Spatial Data Infrastructure (NSDI), the fragmentation hinders the integration and establishment of a homogeneous digital cadastre. In general, the current Kenyan cadastre can be described as a largely incomplete, fragmented, and paper-based patchwork of maps with different accuracies.

For Kajiado, the study area of this chapter, boundaries were derived from aerial imagery and topographic maps of scale 1:50,000, from which, base maps at scale 1:5,000 were prepared, which were enlarged to scale 1:2,500 to produce graphical Registry Index Maps (RIMs). RIMs show the outline of land parcels within a given jurisdiction using general boundaries along visible features. The boundaries' position is only indicative and not legally binding. RIMs and survey plans for urban areas have the highest accuracy specifications of $30 \mathrm{~cm}$ nominal positional accuracy [327]. Different types of RIMs exist that partly allow positional errors of up to $200 \mathrm{~cm}[328,329]$. In Kajiado, RIMs are available in paper-based form (Figure 6.6a). All amendments are made on the original sheet, which leads to consecutive illegibility of the map (Figure 6.6b). During fieldwork, we obtained an understanding of local demarcation practices and captured example boundaries, in order to create a reference dataset for the accuracy analysis of this chapter. Cadastral boundaries are thus, similar to those for the Rwandan data, manually delineated from the UAV orthomosaics based on local knowledge. 


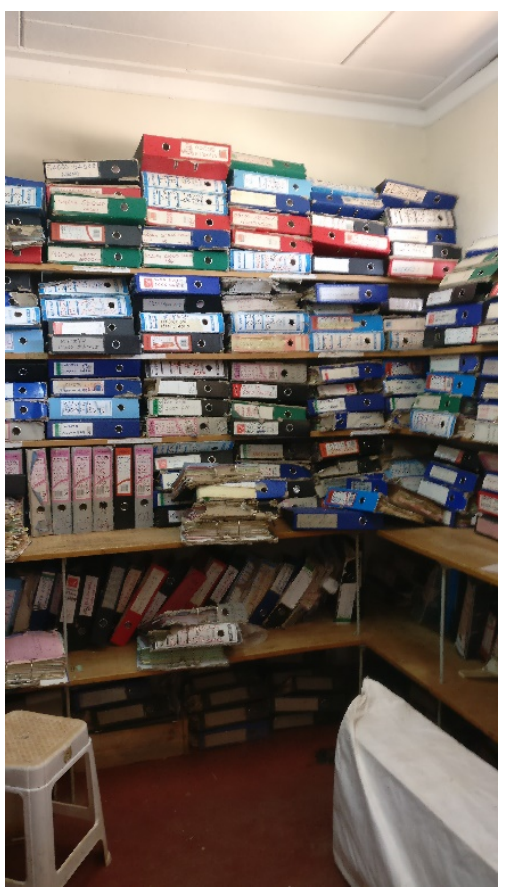

(a)

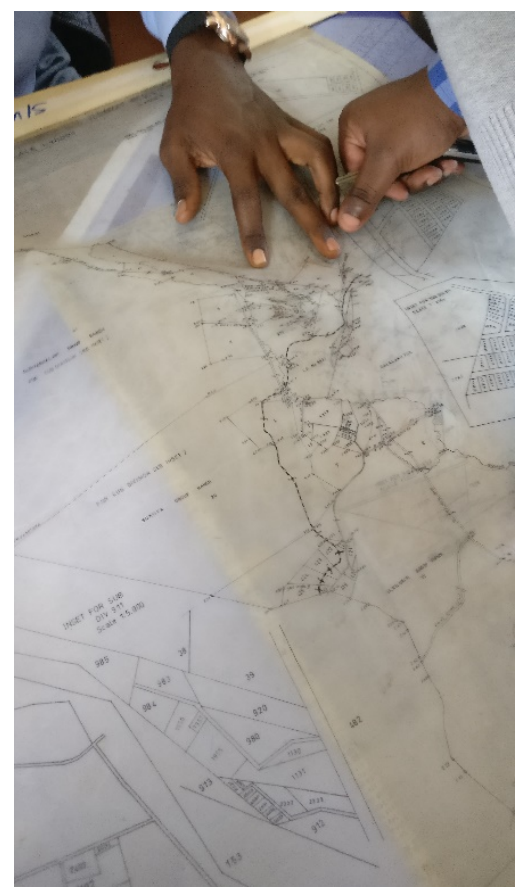

(b)

Figure 6.6. (a) Paper-based cadastral data storage in Kajiado (Kenya), and (b) a paperbased map used in land administration.

\subsubsection{Operational Analysis}

The operational analysis questions when and why the approach is more efficient compared to manual delineation. This is done taking into account observations from the delineation for the accuracy analysis and an additional delineation to provide an overview of successful and challenging delineation cases.

For the peri-urban site, we selected Kajiado, an area close to Nairobi, in which current land administration challenges of urbanization, subdivision, and digitization are present and in which a large portion of cadastral boundaries is visible. Delineating boundaries with indirect surveying from the remote sensing imagery requires knowledge about the boundaries. To recognize boundaries in an image, it helps to be familiar with their appearance on the ground. Therefore, we went to the area for which UAV data were captured and took images of example boundaries. A team of village elders and a local researcher joined us to communicate with land owners when passing and capturing their boundaries. The team explained which objects were typically used to demarcate boundaries and provided insights on local boundary demarcation issues. The fieldwork helped to develop an understanding of the current land administration system available in paper-based form only. 
For the rural site, we selected a Mailua group ranch in Kajiado County. The area is governed by a local pastoralist Masaii community with collectively registered land rights. The local pastoralists live jointly in homesteads around which they undertake pastoralist activities. Neither the homesteads nor the pastoralist activities are currently spatially documented in a formal land administration system. Challenges arise due to increasing subdivision processes without adequate survey control. Visually extractable homesteads' outlines are delineated and attributed with land tenure information derived during fieldwork in the research project.

\subsubsection{Feedback Analysis}

The feedback analysis investigates the approach based on feedback from stakeholders having a background or an affiliation with surveying.

While the former analyses focus on technical aspects, the feedback analysis reveals additional legal, organizational and financial aspects. Considering not only technical feasibility and limitations, but equally the required legal, institutional and financial capacity developments, is essential to implement improvements in land registration [330].

In order to collect feedback on the boundary mapping approach from local land administration stakeholders, three workshops were organized. The design of the workshops was built on insights gained from [323]. Stakeholders were considered relevant when having a background or an affiliation in surveying or related decision making. Three one-day workshops were organized for stakeholders from (i) local government institutions, (ii) NGOs and private companies, as well as (iii) national government institutions (Table 6.4). Each workshop followed the same structure: at the beginning, the research project its4land [3], of which the boundary mapping approach is part of, was introduced (Figure 6.7a). Then, the participants were invited to provide feedback. We demonstrated the interactive delineation and requested participants to delineate example boundaries themselves (Figure 6.7b). We asked the participants which strengths, weaknesses, opportunities, and threats (SWOT) came to their minds when comparing the approach proposed to manual delineation. For the approach proposed, they were asked to consider the entire workflow of image segmentation, boundary classification, and interactive delineation. For manual delineation, they should consider current indirect surveying techniques, i.e., manual delineation on remote sensing imagery. The points on strengths, weaknesses, opportunities and threats were noted individually and afterwards discussed and prioritized in the group (Figure 6.7c). 
Table 6.4. Specifications of workshops organized for feedback collection.

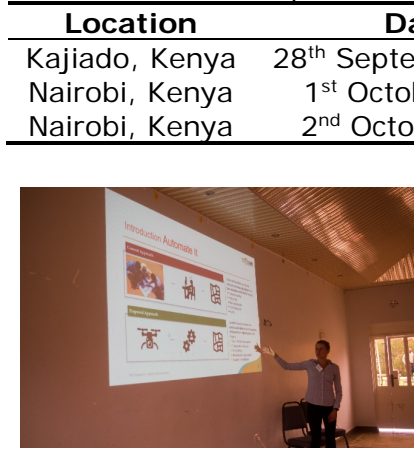

(a) Date Participants

22
32 3

\section{Affiliation}

local government Kajiado NGOs, private companies national government Kenya

Figure 6.7. (a) Explaining concepts of the boundary mapping approach during the workshop introduction. (b) Demonstrating the boundary mapping approach during the interactive feedback session. (c) Discussing strengths, weaknesses, opportunities, and threats (SWOT) of the approach proposed.

\subsection{Results}

\subsubsection{Accuracy Analysis}

The accuracy analysis contains results for the Rwandan and Kenyan study areas, both presented in the following. An insight in the partly overlapping results is provided for the Rwandan data.

For the building outlines in the Rwandan study areas, the training tile contained 1870 lines of which $30 \%$ were labeled as 'boundary' and $70 \%$ as 'not boundary' in Muhoza. In this area, the testing tiles contained 4164 lines that covered 225 buildings in the reference data. In Mukingo, the training tile contained 967 lines of which $23 \%$ were labeled as 'boundary' and $77 \%$ as 'not boundary'. In this area, the testing tiles contained 1344 lines that covered 222 buildings in the reference data.

$317(71 \%)$ out of the 447 building objects could be directly extracted (Figure 6.8a/ b), of which $84 \%$ were extracted with a correctness of $30 \mathrm{~cm}$ (Table 6.5, Table 6.6). For the 86 (19\%) buildings that needed editing, $82 \%$ required minor editing on $<20 \%$ of the outline length, e.g., when the image segmentation did not provide a closed contour encompassing the entire building (Figure 6.8c). The 41 (9\%) buildings that could not be delineated by the approach were mostly missed entirely during image segmentation. An over-segmentation would have allowed delineating more buildings with the approach, making the labeling and later selection of nodes more timeconsuming, due to more useless lines and nodes requiring consideration. The selection of the segmentation parameters was chosen after visual inspection of different settings for the tile as a whole. The inspection aimed to define 
parameters that obtained a delineation for the majority of outlines, while limiting the extraction and fragmentation of useless lines.

For the cadastral boundaries in the Rwandan study areas, the initial step of image segmentation required stronger over-segmentation, as the walls and fences to be extracted had less contrast compared to building outlines. The training tile contained 5831 lines of which 19\% were labeled as 'boundary' and $81 \%$ as 'not boundary' in Muhoza. In Mukingo, the training tile contained 3558 lines of which $15 \%$ were labeled as 'boundary' and $85 \%$ as 'not boundary'. In Mukingo, only a few objects demarcating cadastral boundaries were visible.

The approach could be applied for the delineation of visible cadastral boundaries demarcated through walls and fences (Figure 6.8d/e). The approach is most suited for areas in which a large portion of cadastral boundaries is visible. For the visible parcels, the approach could be used to delineate 37 of $90(41 \%)$ parcels without further editing and 31 of $90(34 \%)$ parcels that required editing (Table 6.5, Table 6.7). Editing was considered only, when the interactive and the editing part together were more efficient than manual delineation. The higher percentage of editing for parcels compared to buildings can be explained by the outlines' complexity: parcel outlines run along different objects with varying characteristics, while building outlines are more consistent. Not all complexities are correctly captured during image segmentation and thus require editing.

When comparing buildings and cadastral boundaries, buildings allow more unambiguous reference data. The data can be captured with precise labeling rules resulting in objects of a specific quantity with an accurate outline. For cadastral boundaries, defining precise labeling rules is more challenging: the reference data should capture visible object outlines that demarcate parcel boundaries. Which objects are considered as visible cadastral boundaries depends on the local context. The cadastral reference may contain parts that are not demarcated through visible objects but can nevertheless be delineated based on the context: if there is a gap between two walls delineating a parcel, the boundary is drawn as the shortest closing of this gap (Figure 6.8g). Similarly, the delineator might delineate the centerline of a wall as a cadastral boundary, while the boundary mapping approach captures the wall's in- or outline (Figure 6.8h). 
Table 6.5. Accuracy assessment of building outlines and cadastral boundaries in Rwandan study areas delineated once manually, once with the approach proposed.

Building Delineation

Boundary Delineation

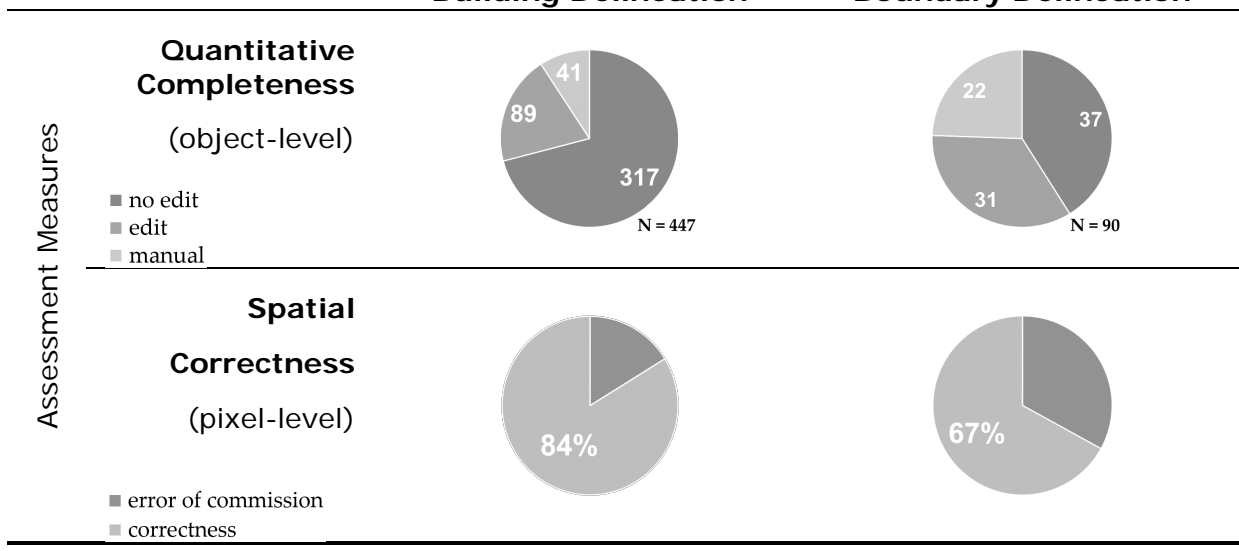

Table 6.6. Detailed results for building delineation in Rwanda.

\begin{tabular}{|c|c|c|c|c|c|c|c|c|}
\hline \multirow{2}{*}{  } & \multicolumn{2}{|c|}{$\begin{array}{c}\text { error of } \\
\text { commission [\%] }\end{array}$} & \multicolumn{2}{|c|}{$\begin{array}{c}\text { buildings } \\
\text { no edit }(\mathrm{N})\end{array}$} & \multicolumn{2}{|c|}{$\begin{array}{l}\text { buildings } \\
\text { edit }(N)\end{array}$} & \multicolumn{2}{|c|}{$\begin{array}{l}\text { buildings } \\
\text { manual (N) }\end{array}$} \\
\hline & Mukingo & Muhoza & Mukingo & Muhoza & Mukingo & Muhoza & Mukingo & Muhoza \\
\hline 1 & 16 & 17 & 103 & 72 & 17 & 39 & 11 & 11 \\
\hline 2 & 16 & 17 & 68 & 74 & 7 & 26 & 16 & 3 \\
\hline & \multicolumn{2}{|c|}{$\overline{\mathrm{x}}=16.5$} & \multicolumn{2}{|c|}{$\Sigma=317$} & \multicolumn{2}{|c|}{$\Sigma=89$} & \multicolumn{2}{|c|}{$\Sigma=41$} \\
\hline
\end{tabular}

Table 6.7. Detailed results for cadastral boundary delineation in Rwanda.

\begin{tabular}{|c|c|c|c|c|c|c|c|c|}
\hline \multirow{2}{*}{ 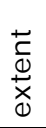 } & \multicolumn{2}{|c|}{$\begin{array}{c}\text { error of } \\
\text { commission [\%] }\end{array}$} & \multicolumn{2}{|c|}{$\begin{array}{l}\text { boundaries } \\
\text { no edit }(\mathrm{N})\end{array}$} & \multicolumn{2}{|c|}{$\begin{array}{l}\text { boundaries } \\
\text { edit }(\mathrm{N})\end{array}$} & \multicolumn{2}{|c|}{$\begin{array}{l}\text { boundaries } \\
\text { manual }(\mathrm{N})\end{array}$} \\
\hline & Mukingo & Muhoza & Mukingo & Muhoza & Mukingo & Muhoza & Mukingo & Muhoza \\
\hline 1 & 22 & 35 & 9 & 14 & 4 & 15 & 4 & 13 \\
\hline 2 & 37 & 36 & 3 & 11 & 0 & 12 & 0 & 5 \\
\hline & \multicolumn{2}{|c|}{$\bar{x}=32.5$} & \multicolumn{2}{|c|}{$\Sigma=37$} & \multicolumn{2}{|c|}{$\Sigma=31$} & \multicolumn{2}{|c|}{$\Sigma=22$} \\
\hline
\end{tabular}

Table 6.8. Detailed results for cadastral boundary delineation in Kenya.

\begin{tabular}{ccccc}
\hline \multirow{2}{*}{} & $\begin{array}{c}\text { error of } \\
\text { commission [\%] }\end{array}$ & $\begin{array}{c}\text { boundaries } \\
\text { no edit }(\mathrm{N})\end{array}$ & $\begin{array}{c}\text { boundaries } \\
\text { edit }(\mathrm{N})\end{array}$ & $\begin{array}{c}\text { boundaries } \\
\text { manual }(\mathrm{N})\end{array}$ \\
\cline { 2 - 5 } & Kajiado & Kajiado & Kajiado & Kajiado \\
\hline 1 & 13 & 15 & 12 & 83 \\
\hline 2 & 24 & 6 & 12 & 83 \\
\hline & $\overline{\mathrm{x}}=18.5$ & $\Sigma=21$ & $\Sigma=24$ & $\Sigma=166$ \\
\hline
\end{tabular}




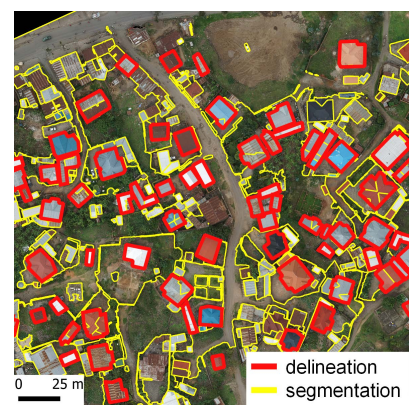

(a)

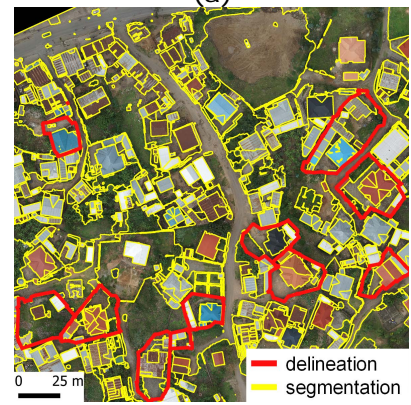

(d)

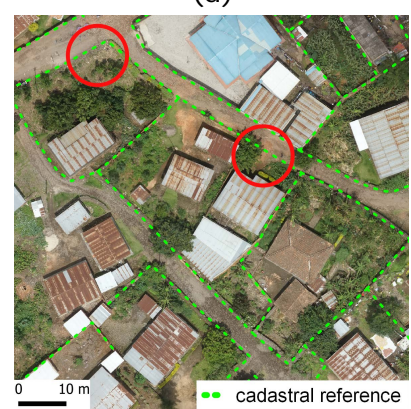

(g)

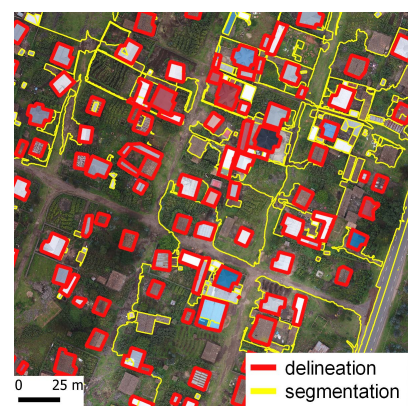

(b)

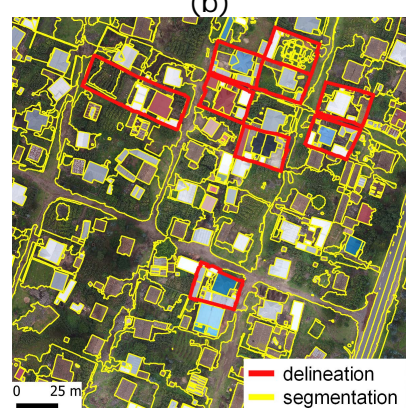

(e)



(h)

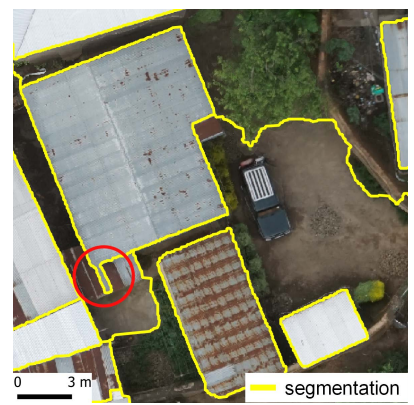

(c)



(f)

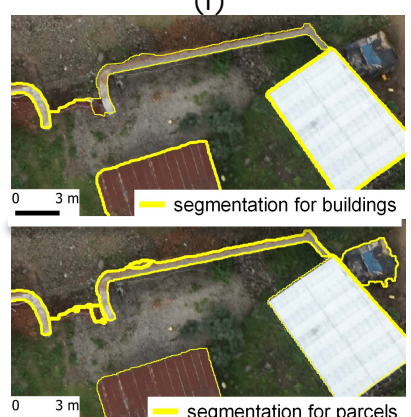

(i)

Figure 6.8. Examples of delineation results for Rwandan study areas: (a/ b) Building delineation. (d/ e) Parcel delineation. (c) Building segmentation requiring editing. (f) Building segmentation from different resolutions. (g) Visible parcels not demarcated by objects but by context. (h) Wall outline and centerline used for parcel delineation. (i) Boundary classification trained to detect buildings (top) and parcels (bottom).

The approach has now been investigated for numerous objects demarcating cadastral boundaries, such as roads [300], buildings, walls, and fences. Which objects demarcate visible cadastral boundaries is location-dependent. Compared to manual delineation, which is the current procedure for indirect surveying [44], the approach obtains the highest time savings for areas in which boundaries are visible, long and curved. This has been shown for roads [300] and is assumed to hold true for agricultural field boundaries as well. The 
walls and fences investigated in this chapter are often covered by vegetation and not built consistently (Figure $\mathbf{6 . 8 g}$ ). Such boundaries are challenging to extract during image segmentation so that the time efficiency for short and straight parts remains comparable to that for manual delineation. The approach could be improved by adding further functionalities: (i) allowing the user to add or move nodes and lines during delineation, (ii) automatically adjusting incorrect corners in the result (Figure 6.8c), and (iii) providing the option to apply the method without the boundary classification step as it adds little value for cases of clear and few boundaries detected through the image segmentation step (Figure $\mathbf{6 . 8 a /} \mathbf{b}$ ).

\subsubsection{Operational Analysis}

The application of each of the approach's workflow steps for Mailua is visualized in Figure 6.9. Two of the five study areas, i.e., those shown in the first two rows of Figure 6.9, were used to train the RF classifier that then predicts boundary likelihoods for lines used during the interactive delineation. Since the UAV data of Mailua did not contain DSM information, corresponding features were not calculated during boundary classification.

Applying the approach in Mailua to delineate homesteads' outlines showed once more the benefits of the approach when delineating curved outlines: To manually delineate the curved outline of the pastoralists' homesteads, each node needs to be clicked. Selecting a location to click, requires detailed zooming in the orthoimage of $5 \mathrm{~cm}$ GSD. Delineating the outline with the approach, requires only two nodes at diagonal locations to be clicked. Selecting these locations can be done, by clicking on two of the automatically extracted nodes on a zoomed out level. The outlines of pastoralists' homesteads were demarcated by piling a specific type of vegetation. The demarcating was of high contrast to its surrounding, seldom covered by other vegetation and established continuously with rare gaps. This allowed effectively applying the approach with little over-segmentation, which resulted in a low number of nodes and lines to be used during the interactive delineation. The extracted outlines are attributed with land tenure information on, e.g., ownership, usage derived in related Sketchmap work in the research project. The approach can thus contribute to the demarcation of pastoralists' homesteads currently not spatially documented in a formal land administration system. 


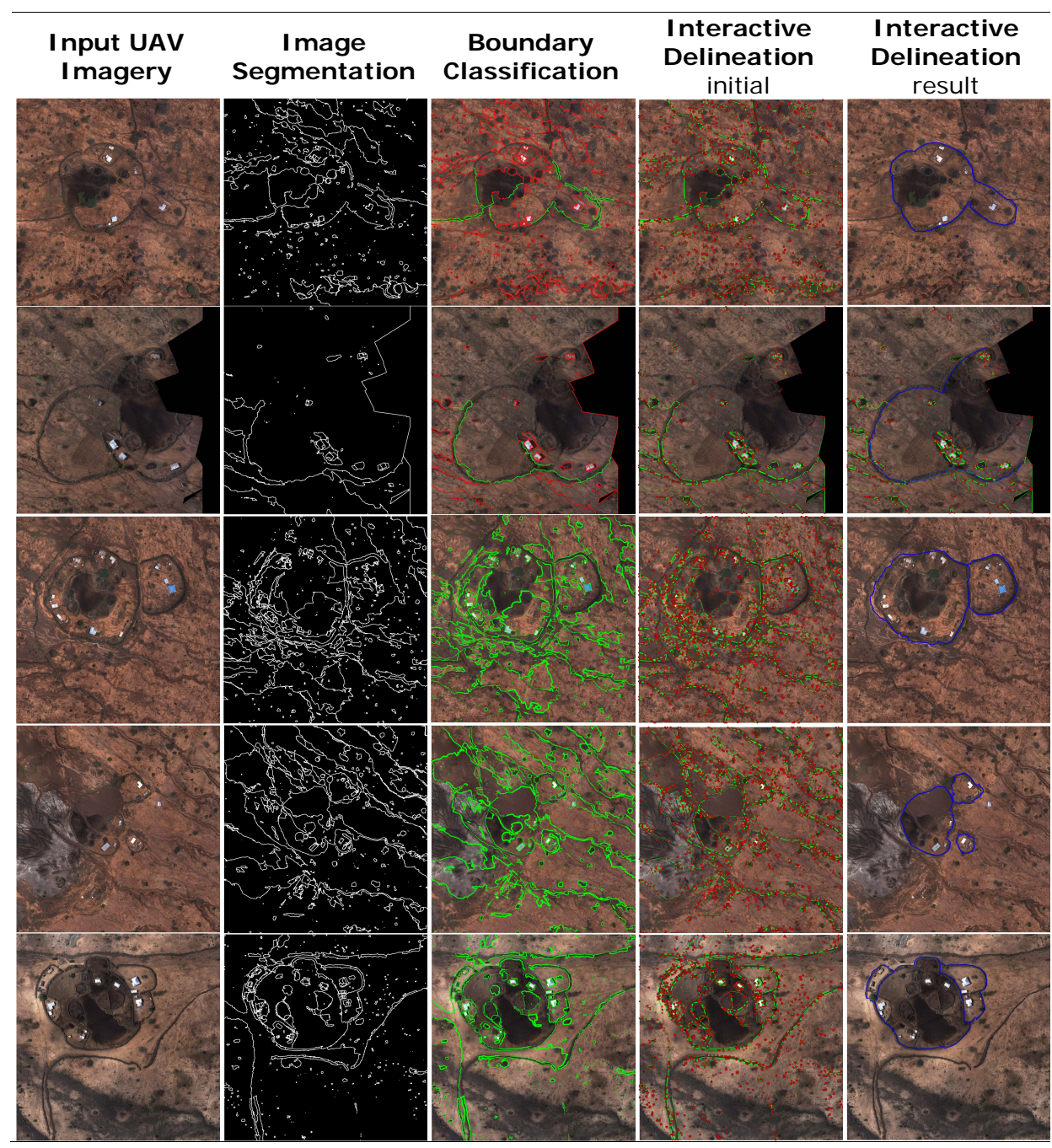

Figure 6.9. Boundary mapping in Mailua: image segmentation, boundary classification, and interactive delineation applied to delineate visible boundaries of pastoralists' homesteads from UAV data.

During fieldwork in Kajiado, we obtained an understanding of local boundary characteristics and demarcation challenges. The letters used in the following refer to Figure 6.10. A majority of boundaries is demarcated by visible objects such as (a) stone walls, (b) corrugated metal fences, (c) vegetation, or (d) ditches. The following examples are extractable from remote sensing imagery, but require local knowledge or context for a correct identification: (h) ditches can be confused with soil erosion, when extracted from imagery alone. (d) Some fences demarcating boundaries are challenging to differentiate from its surrounding. High-resolution DSMs can support the identification of such fences. (f) Beacons demarcate boundary corner points and (g) can be used in 
parallel with linear boundary demarcations, or as control points for hosting measurements. The legal boundary has often remained on the connection of the beacons, instead of on the visible boundary.



(a)

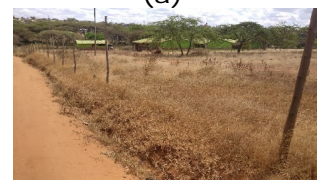

(e)

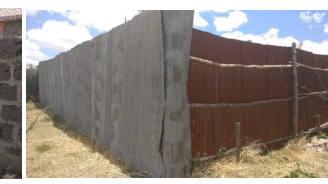

(b)
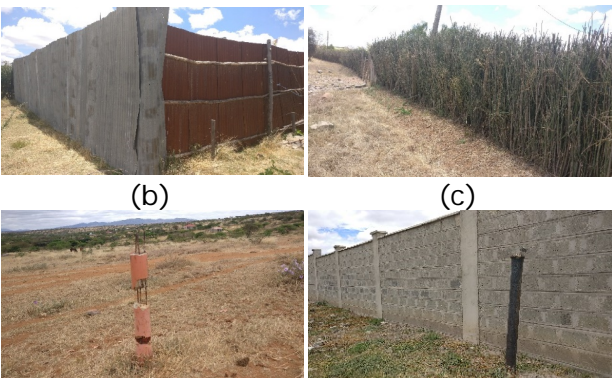

(f) (c)



(g)

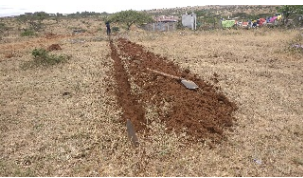

(d)

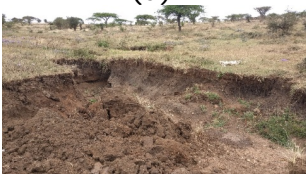

(h)

Figure 6.10. (a-d) Examples of visible boundaries in Kajiado. (e-h) Boundary demarcations challenging to identify correctly from remote sensing imagery.

Based on the local knowledge obtained during fieldwork and the large portion of cadastral boundaries in Kajiado being visible, the boundary mapping approach could be applied to the captured UAV data (Figure 6.11). It took about $30 \mathrm{~min}$ to interactively delineate each example tile of $300 \times 300 \mathrm{~m}$. During application, we observed the following challenges:

(i) I mage segmentation: It appeared difficult to define which level of contrast should be recognized by the image segmentation. An undersegmentation delineates roofs only, while low-contrast outlines of fences and hedges were not extracted (Figure 6.12a). An over-segmentation resulted in outlines of fences and hedges, as well as many superfluous lines, which made the subsequent labeling in (ii) tedious and limited the operability in (iii) due to numerous nodes and lines (Figure 6.12b). In addition, many fences were not continuous or wide enough to be extracted by image segmentation (Figure 6.12e). Applying the approach to accurately extract objects with a precise outline such as roofs is more useful compared to extracting objects with fuzzy outlines such as hedges, for which the delineation requires manual adjustment and local knowledge. Fuzzy boundary demarcations tend to be the ones that are established with less effort, and that stay less permanently. However, accurate outlines of, e.g., roofs also have their flaws: While the roof can be precisely delineated, the actual boundary on the ground can be different or invisible when covered by an overhanging roof or running in between beacons (Figure 6.10f). For the case shown in Figure 6.10g, boundary mapping should focus on extracting and connecting beacons, i.e., the metal poles, rather than delineating the visible boundary, i.e., the massive stone wall. 
(ii) Boundary classification: The lines from (i) were often fragmented (Figure 6.12c), which made manual line labeling into 'boundary' and 'not boundary' during training time-consuming. Joining such fragmented lines to larger ones without losing the corner information would be useful. The benefit of the boundary likelihood to calculate the least-cost-path was limited when no alternative line between two nodes was available (Figure 6.12d). In such cases, in which the user-selected nodes cannot be connected along alternative lines, the least-cost-path does not find the optimal connection, but the only one. Calculating the boundary likelihood is most beneficial to delineate long and curved boundaries [300], and might be omitted when the majority of boundaries is short and along clearly visible object outlines.

(iii) Interactive delineation: Numerous boundaries could be delineated although they were not demarcated by an object: the context made clear that a boundary could be closed without the existence of an object outline, e.g., by closing the gap between two walls delineating a parcel (Figure 6.12e). The same holds true for visible boundaries covered by shadow or vegetation, under which the continuation of, e.g., a fence can be assumed. The nodes, originally created to be user-selected points to be connected along the lines from (i), were useful for the partially required manual delineation and editing of lines: they marked accurate outlines or corner points of objects that the delineation could be snapped to. The line thickness, originally intended to visualize the boundary likelihood from (ii), was useful to quickly identify areas for further delineation without detailed zooming (Figure 6.12f). The line simplification in (iii), originally intended to reduce the number of nodes and to straighten pixel-derived lines, was sometimes too strong, resulting in lines and nodes not fully representing the high-resolution of the input data. Most parcels required partial manual delineation or editing since few were completely demarcated by visible object outlines. Even though not originally designed for manual delineation and editing, the approach simplified these steps due to the described benefits of the suggested nodes and lines. 




(a)

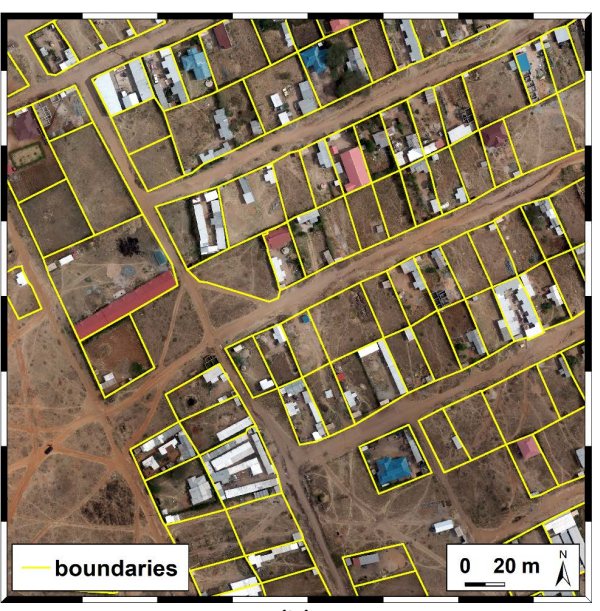

(b)

Figure 6.11. (a/ b) Cadastral boundaries delineated from UAV data with the proposed boundary mapping approach.

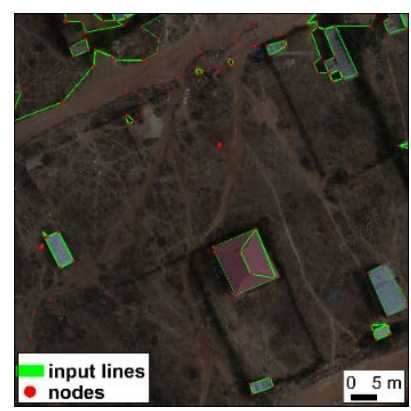

(a)



(d)

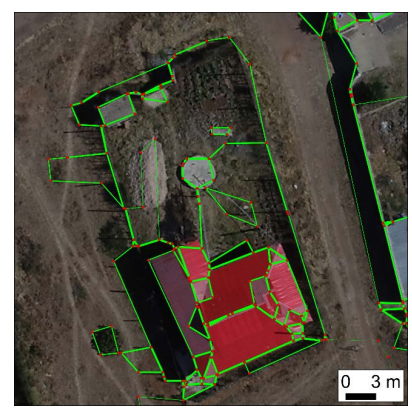

(b)



(e)

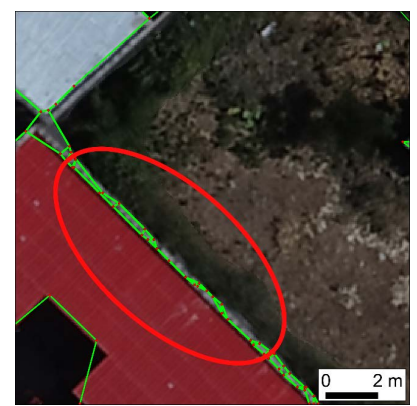

(c)

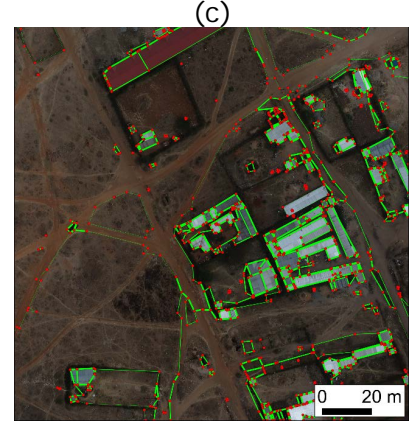

(f)

Figure 6.12. Challenges observed during delineation: (a) under-segmentation, (b) over-segmentation, (c) fragmented segmentation, (d) redundancy of least-costpath calculation, (e) visible boundary not demarcated by objects, but by context, and (f) identification of delineation areas through boundary mapping approach. 


\subsubsection{Feedback Analysis}

The SWOT feedback from the three workshops was grouped according to technology readiness levels (TRL) proposed by the European Commission [331]. TRLs are used to simplify the transition from a scientific idea to a realworld application and enable a consistent and precise discussion on different scales of technical maturity (Figure 6.13). The obtained points on strengths, weaknesses, opportunities and threats are grouped into feedback on the conceptual development (TRL 1-3, Figure 6.14a), the technological development (TRL 4-6, Figure 6.14b), and the operational development (TRL 7-9, Figure 6.14c). The conceptual development relates to the idea and design of the boundary mapping approach. The technological development relates to its current implementation and functioning. The operational development relates to its future applicability in land administration systems.

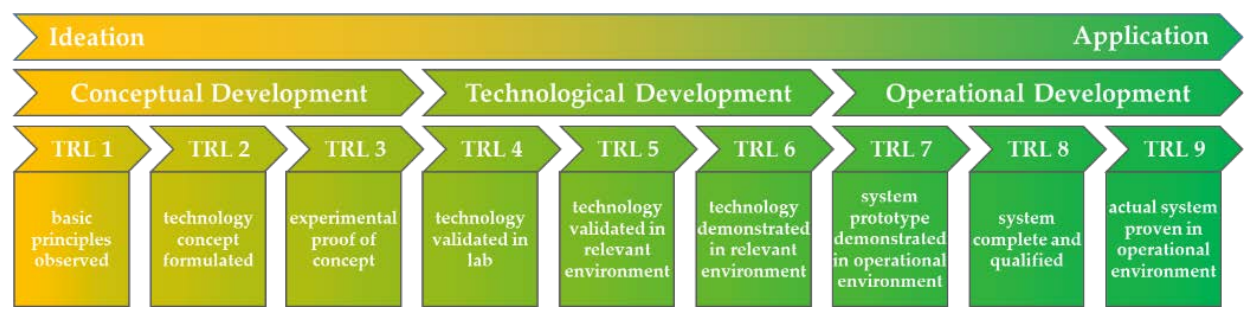

Figure 6.13. Transferring an innovative idea to a successful application along technology readiness levels (TRL) proposed by the European Commission [331]. 


\subsection{Discussion}

The results from the analyses presented before are synthesized in Table 6.9 and discussed in the following.

Table 6.9. Cases for which the approach proposed fails ordered by frequency, and ideas for improvement.

\begin{tabular}{|c|c|c|}
\hline Boundary Type & I ssue Explanation & I mprovement I deas \\
\hline $\begin{array}{l}\text { Fragmented } \\
\text { Figure } 6.12 \mathrm{c}\end{array}$ & $\begin{array}{l}\text { - Over-segmentation } \\
\text { - Tedious line labeling during } \\
\text { classification } \\
\text { - Little overview during } \\
\text { interactive delineation }\end{array}$ & $\begin{array}{l}\text { - J oin fragmented } \\
\text { segmentation results }\end{array}$ \\
\hline $\begin{array}{r}\text { Low-contrast or } \\
\text { fuzzy } \\
\text { Figure } 6.12 \mathrm{a} \\
\end{array}$ & $\begin{array}{l}\text { - Under-segmentation } \\
\text { - Little usability of image } \\
\text { segmentation } \\
\text { - Increase of manual delineation }\end{array}$ & $\begin{array}{l}\text { - Add creation of closed } \\
\text { polygons and geometric } \\
\text { checks } \\
\text { - Simplify moving and } \\
\text { omitting of vertices } \\
\end{array}$ \\
\hline $\begin{array}{r}\begin{array}{r}\text { Short and } \\
\text { straight }\end{array} \\
\text { Figure } 6.12 \mathrm{~d} \\
\end{array}$ & $\begin{array}{l}\text { - Redundancy of boundary } \\
\text { likelihood and least-cost-path } \\
\text { calculation }\end{array}$ & $\begin{array}{l}\text { - Make usage of boundary } \\
\text { likelihood optional }\end{array}$ \\
\hline $\begin{array}{r}\text { Multi-type } \\
\text { Figure } 6.12 b\end{array}$ & $\begin{array}{l}\text { - Manual delineation more } \\
\text { efficient than combination of } \\
\text { interactive and manual } \\
\text { delineation } \\
\text { - Nodes and lines are useful to } \\
\text { snap delineation to }\end{array}$ & $\begin{array}{l}\text { - Couple interactive and } \\
\text { manual delineation more } \\
\text { efficiently }\end{array}$ \\
\hline $\begin{array}{r}\text { Context- } \\
\text { dependent } \\
\text { Figure } 6.8 \mathrm{~g} \\
\text { Figure } 6.12 \mathrm{e}\end{array}$ & $\begin{array}{l}\text { - Increase of manual delineation } \\
\text { - Nodes and lines are useful to } \\
\text { snap delineation to }\end{array}$ & $\begin{array}{l}\text { - Add creation of closed } \\
\text { polygons and geometric } \\
\text { checks } \\
\text { - Simplify moving and } \\
\text { omitting of vertices } \\
\text { - Incorporate local } \\
\text { demarcation practices } \\
\text { from existing maps }\end{array}$ \\
\hline $\begin{array}{r}\begin{array}{r}\text { Knowledge- } \\
\text { dependent }\end{array} \\
\text { Figure } 6.8 \mathrm{~h} \\
\end{array}$ & $\begin{array}{l}\text { - Increase of manual delineation } \\
\text { - Increased need for ground- } \\
\text { level images }\end{array}$ & $\begin{array}{l}\text { - Incorporate local } \\
\text { demarcation knowledge in } \\
\text { classifier } \\
\text { - Incorporate recursive } \\
\text { learning }\end{array}$ \\
\hline $\begin{array}{l}\text { Covered by } \\
\text { vegetation }\end{array}$ & $\begin{array}{l}\text { - Little usability of image } \\
\text { segmentation } \\
\text { - Increase of manual delineation }\end{array}$ & $\begin{array}{l}\text { - I ncorporate LiDAR data or } \\
\text { high-resolution DTMs } \\
\text { - Exclude vegetation } \\
\text { through semantic } \\
\text { recognition }\end{array}$ \\
\hline $\begin{array}{r}\text { I nvisible } \\
\text { Figure } 6.12 \mathrm{a} \\
\end{array}$ & $\begin{array}{l}\text { - Little usability of image } \\
\text { segmentation } \\
\text { - Increase of manual delineation }\end{array}$ & $\begin{array}{l}\text { - Incorporate local } \\
\text { demarcation practices } \\
\text { from existing maps }\end{array}$ \\
\hline $\begin{array}{r}\text { Beacons as } \\
\text { boundaries }\end{array}$ & $\begin{array}{l}\text { - No recognition of beacons } \\
\text { through image segmentation }\end{array}$ & $\begin{array}{l}\text { - Include beacon recognition } \\
\text { and linkage }\end{array}$ \\
\hline
\end{tabular}


The results obtained show once more that advantages of automated cadastral mapping depend mainly on the quality of automatically extracted features and their match with cadastral boundaries. As for the automatically extracted features, the visibility of features to be extracted is crucial for the usefulness of lines generated through image segmentation. As for their match with cadastral boundaries, the concept of visible boundaries can be questioned: most indirect surveying studies are based on the assumption that physical objects, e.g., fences, walls, hedges or roads, demarcate visible cadastral boundaries [42-44,304]. This assumption neglects the following: the correct identification of physical objects often requires additional local knowledge (Figure 6.10e-h). Even after correct identification, the outlines of physical objects require further consideration to be transferred into cadastral boundaries: the context often provides more insights than the object outlines themselves. While a delineator closes a gap between two walls demarcating a parcel, selects the in- or outside of a wall as a boundary, or assumes a rectangular shape of similar size in a row of plots, current automated boundary mapping approaches do not incorporate such knowledge. A delineator has more skills than delineating the outlines of visible linear features.

The development of automated approaches in cadastral mapping should thus not only rely on extracting objects but also on closing boundaries based on local knowledge and context. This information is not inherent in the concept of the visible boundary, but it is extractable from remote sensing imagery. To further develop automated cadastral mapping in indirect surveying, we suggest considering the extractable boundary rather than the visible boundary alone (Figure 6.15).

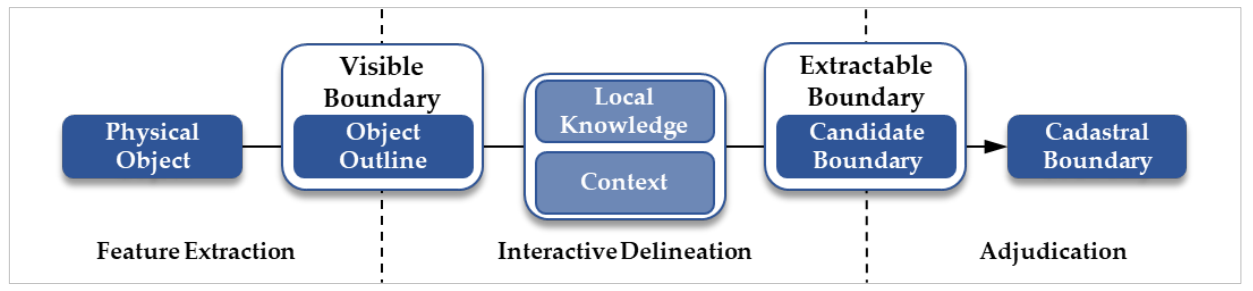

Figure 6.15. From physical object to cadastral boundary: reformulated boundary concepts for indirect surveying.

In general, cadastral boundary mapping from remote sensing data, known as indirect surveying, depends on the percentage of cadastral boundaries being extractable and not necessarily being visible. We observed that a majority of boundaries is extractable, but not necessarily visible, i.e., demarcated by a physical object. While we suggested before to consider the percentage of boundaries being visible before choosing a surveying approach, we now recommend to consider the percentage of boundaries being extractable. Our approach that couples a machine-based automatic feature extraction with a 
delineator-based interactive delineation can be used to map extractable boundaries. Current limitations are present on a conceptual, technological and operational level in each workflow step of image segmentation, boundary classification, and interactive delineation. The presented ideas on improving upon these limitations can serve as a basis for future work. Furthermore, local knowledge could be incorporated by considering ground-level images (Figure 6.10) in addition to the UAV data. Context knowledge could be incorporated by adding learning phases to the workflow.

Given the complexity of cadastral boundaries, automating their delineation remains challenging: the variability of objects and extraction methods reflects the problem's complexity, consisting of extracting different objects with varying characteristics. These circumstances impede the compilation of a generic model for a cadastral boundary and thus the development of a generic method. These remarks come back to the limitations of general boundaries: no standardized specifications exist for boundary features, boundaries are often not marked continuously and maintained poorly [328].

Despite considerable progress in object extraction and classification from remote sensing data, Höhle points out that a gap often remains between the result of an automatic approach and the desired map product [332]. Similarly, Chen et al. constitute a considerable amount of manual intervention required in most existing methods [333]. An alternative to our current approach may be a deep learning based approach. Significant progress has been achieved in object extraction through deep learning [334-336]. For building extraction, deep learning has shown substantial improvements with high accuracies achieved mostly for buildings of consistent and regular roof sizes and shapes [333]. Applying similar approaches for boundary extraction is however not trivial: deep learning approaches require large amounts of training data, thus cadastral data and remote sensing data. Current governmental open data initiatives to publically share such data [337,338] and open-service data portals for aerial imagery [339] could contribute to generating sufficient training data and thus in developing deep learning approaches for automated cadastral mapping.

In any case, the delineation cannot be fully automated at the current state since the extracted outlines require (legal) adjudication and incorporation of local knowledge from human operators to create final cadastral boundaries. I mage-based approaches offer the potential to automatically extract use rights, which do not necessarily represent legal rights. These circumstances limit the scope of automated approaches. Therefore, future studies should focus on the interactive part that bridges the gap between automatically generated results and the desired product, i.e., cadastral boundaries. This would promote the paradigm shift towards cadastral intelligence that integrates human-based 
expert knowledge with automatically generated machine-based knowledge. Additionally, future studies should provide approaches to capture requirements from existing technical, legal, financial, and institutional frameworks to be considered when aiming to implement innovative cadastral mapping procedures successfully.

\subsection{Conclusion}

This chapter focusses on a previously developed boundary mapping approach [300]. The approach supports the delineation of objects by automatically retrieving information from UAV-based RGB and DSM data that is used to guide an interactive boundary delineation. In this chapter, we introduce an improved version, in which the steps of image segmentation and line extraction have been combined, the features used during classification have been optimized, the interactive delineation has been redesigned to be more intuitive, and the entire workflow has undergone refactoring and restructuring for a more stable and efficient applicability. Subsequently, we describe the approach's current functioning, demonstrate results obtained in different scenarios, and synthesize user feedback.

Specific bottlenecks on a conceptual, technological and operational level are listed and can serve as a basis for future work. The insights from this chapter allow reformulating current boundary concepts in indirect surveying, in order to develop automated feature extraction for UAV-based cadastral mapping further: instead of focusing on the visible boundary comprising outlines of physical objects, automated cadastral mapping should focus on the extractable boundary that incorporates local knowledge and context. On the one hand this calls to incorporate deep or recursive learning. On the other hand, we observed that automating cadastral mapping dealing with sensitive land rights can only be successful, when the interactive part that bridges the gap between automatically generated results and the final cadastral boundary is designed and implemented in correspondence to surveyors' needs. Our work revealed limitations of the current approach and ideas for improvements to be addressed in future work to advance the current approach in terms of efficiency and acceptance. 


\section{Deep Learning for Boundary Line Classification in Cadastral Mapping ${ }^{\star}$}

* This chapter is based on:

340. Crommelinck, S.; Koeva, M.; Yang, M.Y.; Vosselman, G. Application of Deep Learning for Delineation of Visible Cadastral Boundaries from Remote Sensing Imagery. Remote Sensing 2019, 11, 1-22. 


\begin{abstract}
Cadastral boundaries are often demarcated by objects that are visible in remote sensing imagery. Indirect surveying relies on the delineation of visible parcel boundaries from such images. Despite advances in automated detection and localization of objects from images, indirect surveying is rarely automated and relies on manual on-screen delineation. We have previously introduced a boundary delineation workflow comprising image segmentation, boundary classification, and interactive delineation. In this study, we improve each of these steps. For image segmentation, we limit over-segmentation by reducing the number of segment lines by $80 \%$ through filtering. For boundary classification, we show how Convolutional Neural Networks (CNN) can be used for boundary line classification and achieve $71 \%$ accuracy. For interactive delineation, we develop additional delineation functionalities. We apply our approach to UAV and aerial imagery of $0.02-0.25$ m resolution from Kenya, Rwanda and Ethiopia. We show that it is more effective in terms of clicks and time compared to manual delineation for parcels surrounded by visible boundaries. Strongest advantages are obtained for rural scenes delineated from aerial imagery, where the delineation effort per parcel requires $38 \%$ less time and $80 \%$ fewer clicks compared to manual delineation.
\end{abstract}




\subsection{Introduction}

Cadastral mapping is considered as the most expensive part of the land administration system [7]. There is a clear need for innovation for fast, accurate and cost-effective land rights mapping in order to provide tenure security and sustainable governance. We previously proposed automating indirect surveying for cadastral mapping by the following workflow [300]: (i) image segmentation to extract visible object outlines, (ii) boundary classification to predict boundary likelihoods for extracted segment lines, and (iii) interactive delineation to connect these lines based on the predicted boundary likelihood.

In this study, we investigate improvements in all three steps (Figure 7.1). First, for step (i), we filter out small segments to reduce over-segmentation. Second, for step (ii), we replace hand-crafted features and line classification based on Random Forest (RF) by Convolutional Neural Networks (CNNs). Finally, for step (iii), we introduce more intuitive and comprehensive delineation functionalities.
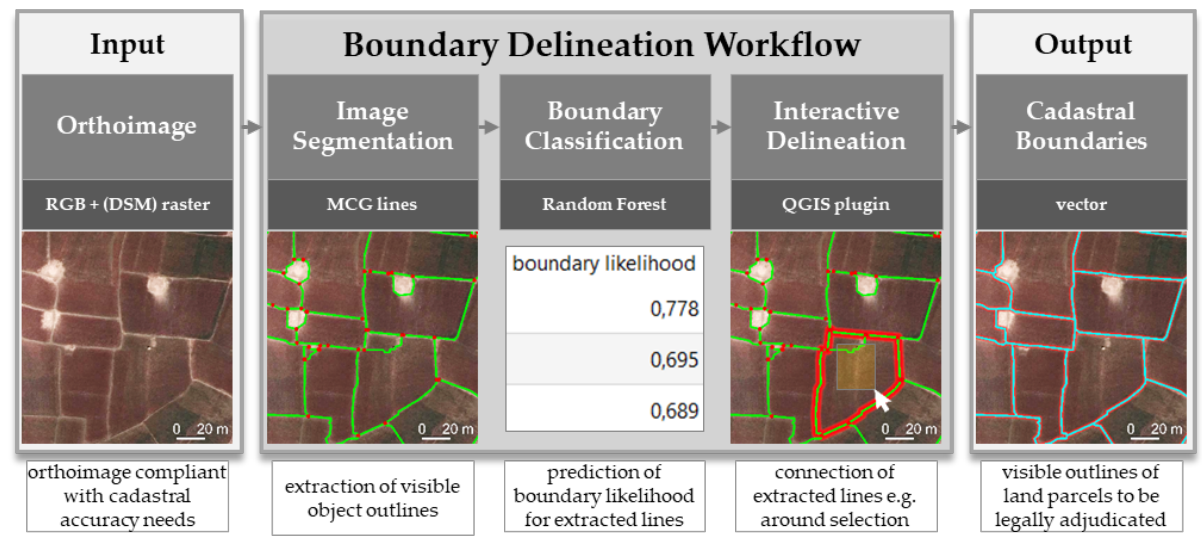

Figure 7.1. Boundary Delineation workflow proposed to improve indirect surveying. This study optimizes image segmentation, questions whether Random Forest (RF) or Convolutional Neural Networks (CNN) are better suited to derive boundary likelihoods for visible object outlines, and introduces additional functionalities for the interactive delineation.

CNNs are one of the most popular and successful deep networks for image interpretation tasks. They are proven to work efficiently to identify various objects in remote sensing imagery [336,341-344]. Comprehensive overviews contextualizing the evolution of deep learning and CNNs in geoscience and remote sensing are provided in $[335,345]$. In essence, CNNs are neural networks that incorporate the convolution and pooling operation as a layer. CNNs have been characterized by five concepts [346]: 
(i) Convolution operation increases the network's simplicity, which makes training more efficient.

(ii) Representation learning through filters requires the user to engineer the architecture rather than the features.

(iii) Location invariance through pooling layers allows filters to detect features dissociated from their location.

(iv) Hierarchy of layers allows learning of abstract concepts based on simpler concepts.

(v) Feature extraction and classification are included in training, which eliminates the traditional machine learning need for hand-crafted features, and distinguishes $\mathrm{CNN}$ as a deep learning approach.

In deep learning, there are two approaches to train a CNN: from scratch or via transfer learning [347]. When trained from scratch, all features are learned from data to be provided, which demands large amounts of data and comes with a higher risk of overfitting. An overfitted network can make accurate predictions for a certain dataset, but fails to generalize its learning capacity for another dataset. With transfer learning, part of the features are learned from a different, typically large dataset. These low-level features are more general and abstract. The network has proven excellence for a specific application. Its core architecture is kept and applied to a new application. Only the last convolution block is trained on specific data of the new application resulting in specialized high-level features. Transfer learning requires learning fewer features and thus fewer data. In our study, we investigate transfer learning an existing CNN for cadastral mapping.

Our new functionalities for the interactive delineation address cases for which the boundary classification fails or is not necessary. Boundary classification comes into play in cases of over-segmentation, when many object outlines exist. Then, the delineator has to choose from a collection of lines which ones demarcate the cadastral boundary. Support comes from the lines' boundary likelihood predicted by RF or CNN. Sometimes, the prediction is not correct or only one outline surrounds a parcel. Then functionalities other than the leastcost-path searching optimal boundary likelihoods are required. In this study, we introduce functionalities that allow connecting image segmentation lines to cadastral boundaries regardless of their boundary likelihood.

\subsection{Materials and Methods}

\subsubsection{Image Data}

In this study, an aerial image of $0.25 \mathrm{~m}$ Ground Sample Distance (GSD) of a rural scene in Ethiopia is used. The cadastral reference data cover $33 \mathrm{~km}^{2}$ containing 9,454 plots with a median size of $2,500 \mathrm{~m}^{2}$. For a later assessment, 
in which we compare our approach to on-screen manual delineation, additional UAV data from Kenya and Rwanda is used. Capture and processing are described in [348].

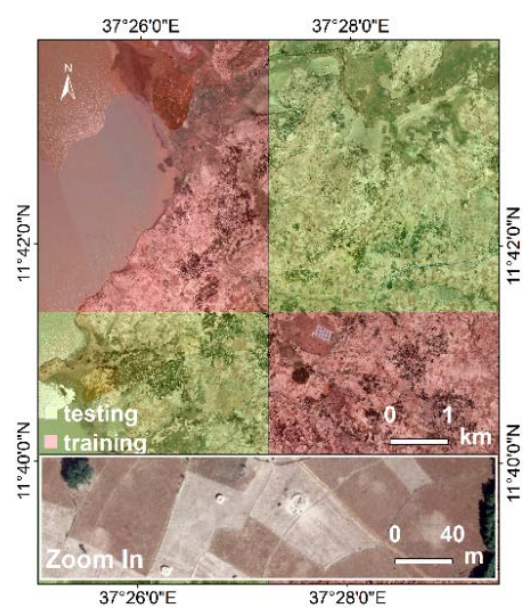

(a)

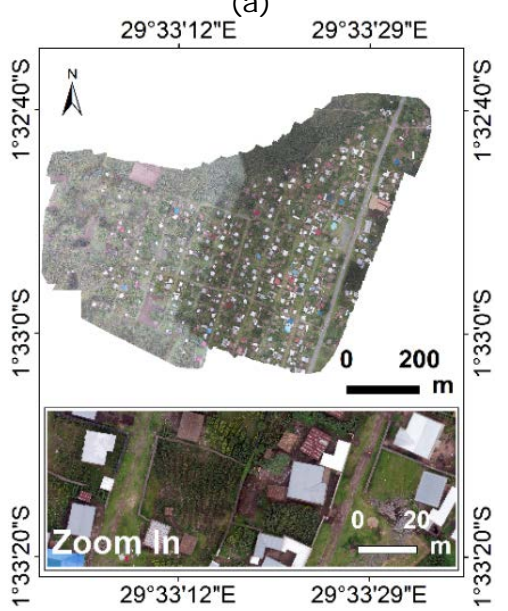

(c)

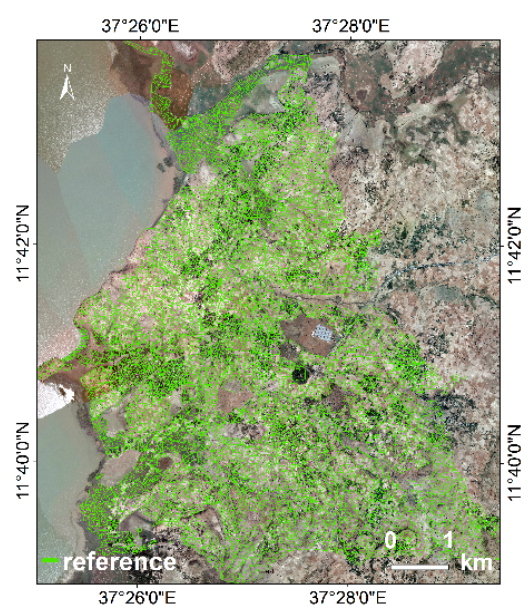

(b)

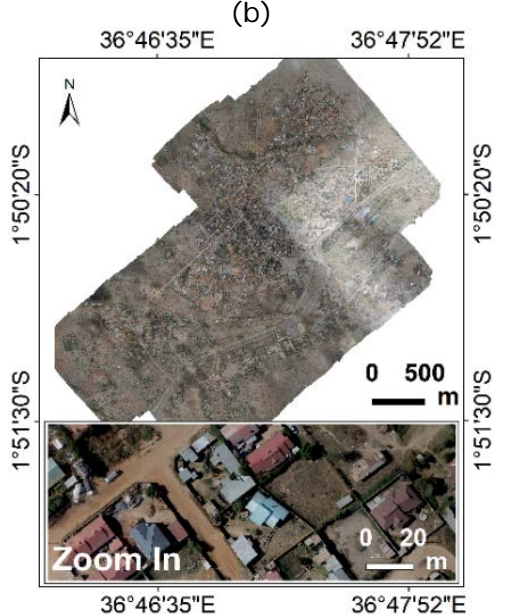

(d)

Figure 7.2. (a) Aerial image of $0.25 \mathrm{~m} \mathrm{GSD}$ for a rural scene in Ethiopia, divided into areas for training and testing our approach before comparing results to (b) cadastral reference. UAV images for peri-urban scenes in (c) Rwanda (0.02 m GSD), and (d) Kenya ( $0.06 \mathrm{~m} \mathrm{GSD}$ ) to compare automated to manual delineation.

\subsubsection{Boundary Mapping Approach}

The boundary mapping approach refers to the one described in chapter 7 . In the following, modifications and the data-dependent implementation of the three workflow steps are described. The source code is publically available under [305]. 
(i) I mage segmentation is based on Multiresolution Combinatorial Grouping (MCG) [117], which delivers closed contours capturing the outlines of visible objects. To run the original MCG implementation, the Ethiopian aerial image is tiled to 20 tiles of $8000 \times 8000$ pixels. The parameter $k$ regulating over- and under-segmentation is set to produce oversegmentation $(k=0.1)$. This setting creates outlines around the majority of visible objects. Tests with parameters ( $k=0.3$ and $k=0.5)$ resulting in less over-segmentation show that visible object outlines are partly missed, while irrelevant lines around small objects are still produced. To reduce the number of irrelevant lines produced through over-segmentation, the lines are simplified through filtering (Figure 7.3): lines around areas smaller than $30 \mathrm{~m}^{2}$ are merged to the neighboring segments, which reduces the line count by $80 \%$ to 600,000 lines. According to our visual inspections, this post-processing removes artefacts in the segmentation results and keeps outlines of large objects being more relevant for cadastral mapping. For the high-resolution data from Rwanda and Kenya, we proceed similarly by tiling the data and setting $\mathrm{k}=0.4$ and $\mathrm{k}=0.3$, respectively.


Figure 7.3. MCG image segmentation lines around visible objects before and after simplification reducing the line count by $80 \%$.

(ii) Boundary classification is applied to the post-processed 600,000 MCG lines. We investigate two machine learning approaches to derive the boundary likelihood per MCG line: Random Forest (RF) and Convolutional Neural Networks (CNN). Both require labelling of training data as 'boundary' and 'not boundary'. The training data for RF consist of lines, that for CNN of image tiles. For both approaches, the cadastral reference is buffered by a radius of $0.4 \mathrm{~m}$. This size accounts for inaccuracies in the cadastral reference and the orthoimage, enlarges the number of 'boundary' samples, and is identical to the one applied to derive hand-crafted RF features. For both approaches, the ratio between training and testing data is set to $50 \%$. The number of 'boundary' and 'not boundary' training samples is balanced to $1: 1$ by randomly under-sampling 'not boundary' tiles (Table 7.1). The areas for training and testing are randomly selected and large to minimize the number of lines at the borders of each area that are clipped and of limited use for further analysis (Figure 7.2). The boundary likelihood predicted by both approaches represents the probability $(\hat{y})$ of a line being 'boundary': 


$$
\text { boundary likelihood }[0 ; 1]=\hat{y}_{\text {boundary }}
$$

Table 7.1. Distribution of training and testing data for boundary classification based on Random Forest (RF) and Convolutional Neural Networks (CNN)

\begin{tabular}{rcccc}
\hline & \multicolumn{2}{c}{ RF classification } & \multicolumn{2}{c}{ CNN classification } \\
\cline { 2 - 5 } & \multicolumn{2}{c}{ number of lines } & \multicolumn{2}{c}{ number of tiles } \\
\hline Label & training & testing & training & testing \\
\hline 'boundary' & $12,280(50 \%)$ & $9,742(3 \%)$ & $35,643(50 \%)$ & $34,721(4 \%)$ \\
\hline 'not boundary' & $12,280(50 \%)$ & $280,108(97 \%)$ & $34,665(50 \%)$ & $746,349(96 \%)$ \\
\hline$\Sigma$ & 24,560 & 289,850 & 70,308 & 781,070 \\
\hline
\end{tabular}

RF classification is applied as described in chapter 7 and shown in Figure 7.4. Instead of manually labelling lines for training, a line is now automatically labelled as 'boundary' when it overlaps with the cadastral reference buffer of $0.4 \mathrm{~m}$ by more than $50 \%$. This value aligns with the threshold at which a CNN-derived result is labelled as 'boundary' or 'not boundary'. Since no DSM information is available for the study area, the feature dsm_grad is not calculated.

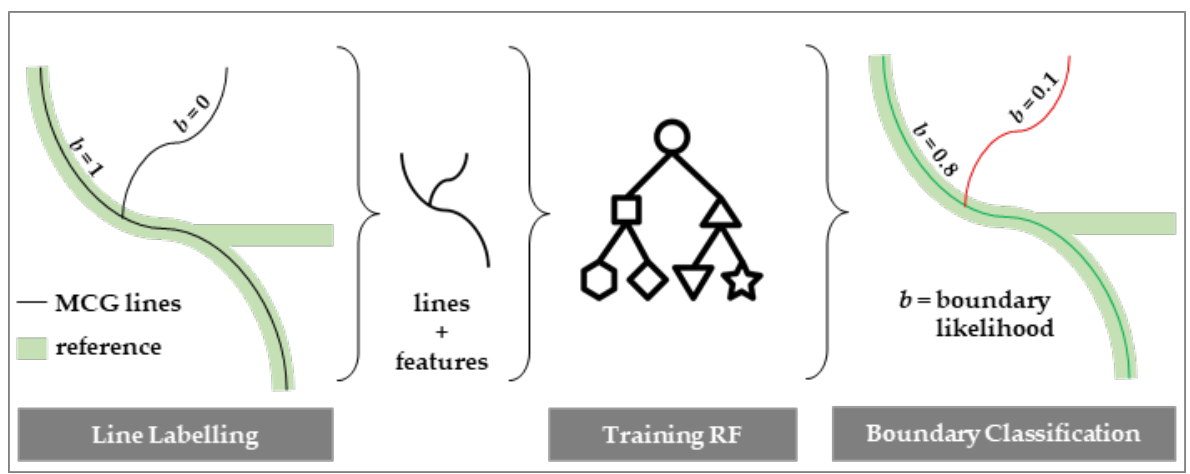

Figure 7.4: Boundary line classification based on Random Forest (RF) to derive boundary likelihoods for MCG lines.

CNN classification is investigated by training state-of-the-art tile-based CNNs (Figure 7.5). We reformulate our problem of generating boundary likelihoods for MCG lines to be solvable by a tile-based CNN as follows: at first, image tiles of $224 \times 224$ pixels centered on an MCG line are cropped from the orthoimage. $224 \times 224 \times 3$ is the standard size of images required by the used CNN. A tile is labelled as 'boundary' if the center pixel covering an MCG line overlaps with the cadastral reference buffer. A tile is created every $5 \mathrm{~m}$ along an MCG line. Decreasing this distance would increase overlap and thus redundancy of image content per tile. Increasing this distance would reduce the number of tiles and thus the number of training data. With these settings, we generate 1.5 million tiles surrounding MCG pixels of which $5 \%$ are labelled as 'boundary' and $95 \%$ as 'not boundary'. After training, the CNN predicts boundary likelihoods for unseen testing areas (Figure 7.2a). The likelihoods 
of all tiles per MCG line are averaged based on the $97^{\text {th }}$ percentile. This value aligns with the distribution of 'boundary' and 'not boundary' lines in the training data (Table 7.1). We use a pre-trained CNN architecture. We apply transfer learning by adding additional trainable layers: a global spatial average pooling layer, a fully connected layer with rectified linear unit (ReLU) activation, a dropout layer, and a logistic layer with softmax activation. Only these last layers are trainable. We investigate using different pre-trained CNN architectures, including VGG [349], ResNet [350], Inception [351] , Xception [352], MobileNet [353] and DenseNet [354], as well as different hyperparameter settings on the learning optimizer, the depth of the fully connected layer, and the dropout rate.



Figure 7.5. Boundary line classification based on Convolutional Neural Networks (CNN) to derive boundary likelihoods for MCG lines.

Interactive delineation supports the creation of final cadastral boundaries. In comparison to chapter 7, we now include more functionalities to delineate parcels (Table 7.2) and redesigned the Graphical User Interface (GUI). The interactive delineation is implemented in the open source geographic information system QGIS [296] as BoundaryDelineation plugin [306]:

Table 7.2. Delineation functionalities of BoundaryDelineation QGIS plugin

\begin{tabular}{ll}
\hline \multicolumn{1}{c}{ Functionality } & \multicolumn{1}{c}{ Description } \\
\hline Connect around selection & $\begin{array}{l}\text { Connect lines surrounding a click or } \\
\text { selection of lines (Figure 7.6a/ b) }\end{array}$ \\
\hline Connect lines' endpoints & $\begin{array}{l}\text { Connect endpoints of selected lines to a } \\
\text { polygon regardless of MCG lines } \\
\text { (Figure 7.6c) }\end{array}$ \\
\hline Connect along optimal path & $\begin{array}{l}\text { Connect vertices along least-cost-path } \\
\text { based on a selected attribute, e.g., } \\
\text { boundary likelihood (Figure 7.6d) }\end{array}$ \\
\hline Connect manual clicks & $\begin{array}{l}\text { Manual delineation with the option to snap } \\
\text { to input lines and vertices }\end{array}$ \\
\hline Update edits & Update input lines based on manual edits \\
\hline Polygonize results & $\begin{array}{l}\text { Convert created boundary lines to } \\
\text { polygons }\end{array}$ \\
\hline
\end{tabular}




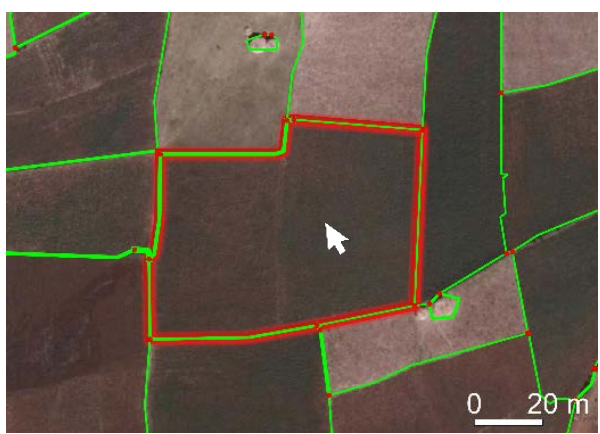

(a)

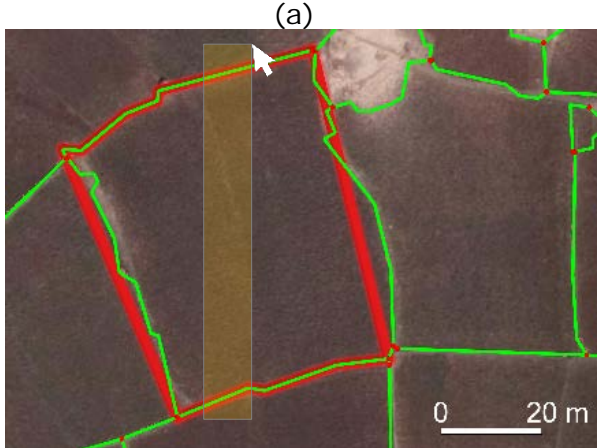

(c)

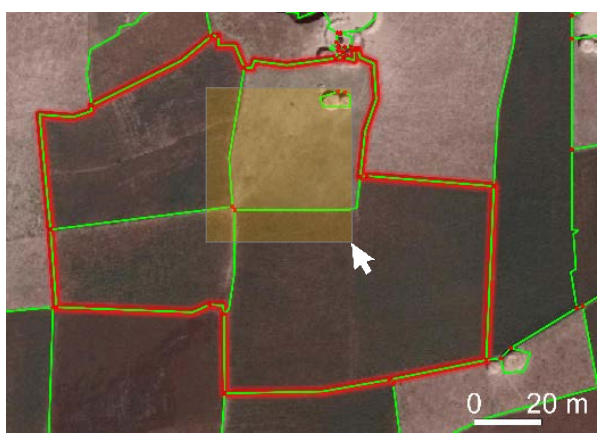

(b)

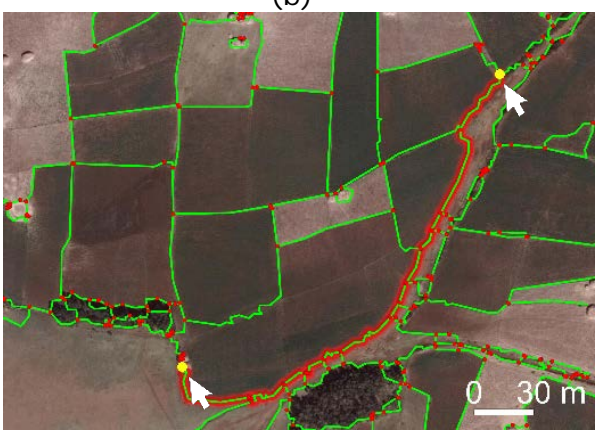

(d)

Figure 7.6: Interactive delineation functionalities: (a) connect lines surrounding a click, or (b) a selection of lines. (c) Close endpoints of selected lines to a polygon. (d) Connect lines along least-cost-path.



Figure 7.7. Interface of open source QGIS BoundaryDelineation plugin [306] developed to guide interactive delineation functionalities.

\subsubsection{Accuracy Assessment}

The accuracy assessment investigates multiple aspects of our workflow each requiring a different analysis: 
CNN Architecture: this analysis aims to optimize the CNN architecture by considering loss and accuracy for training and validation data per epoch. The curves for training loss and validation loss, as well as for training accuracy and validation accuracy, are expected to converge with incremental epochs. Loss is the summation of errors made for each example in training and should be minimized. We use cross-entropy loss that increases as the predicted probability $\left(\hat{y}_{i}\right)$ diverges from the actual label $\left(y_{i}\right)$ :

$$
\text { cross }- \text { entropy loss }=-\left(y_{i} \log \left(\hat{y}_{i}\right)+\left(1-y_{i}\right) \log \left(1-\hat{y}_{i}\right)\right)
$$

All predictions $<0.5$ are considered as 'not boundary', those $>=0.5$ as 'boundary'. This results in a confusion matrix showing the number of tiles being False Positive (FP), True Positive (TP), False Negative (FN), and True Negative (TN). From this matrix, the accuracy is derived as the sum of correctly classified tiles divided by all tiles:

$$
\operatorname{accuracy}[0 ; 1]=\frac{\mathrm{TP}+\mathrm{TN}}{\mathrm{TP}+\mathrm{FP}+\mathrm{FN}+\mathrm{TN}}
$$

RF vs. CNN Classification: this analysis compares the boundary likelihood obtained through RF and CNN to the percentage to which an MCG line overlaps with the cadastral reference. Both are buffered with a radius of $0.4 \mathrm{~m}$. The area of their overlap in relation to the entire MCG buffer area represents the percentage of overlap:

$$
\text { overlap }[0 ; 1]=\frac{\text { area }_{M C G-\text { buffer }} \cap \text { area }_{\text {cadastral-buffer }}}{\text { area }_{M C G-\text { buffer }}}
$$

We investigate whether lines that should get a boundary likelihood $>0$, i.e., those that fall within the cadastral reference buffer, are assigned a boundary likelihood $>0$ :

$$
\text { recall }[0 ; 1]=\frac{\mathrm{TP}}{\mathrm{TP}+\mathrm{FN}}
$$

Then, we check whether the assigned boundary likelihood is valid, i.e., whether it is equal to the line's overlap with the cadastral reference buffer. This is indicated by the precision that captures the ratio of lines having a boundary likelihood that aligns with overlap to the sum of lines having a correct or too positive boundary likelihood:

$$
\text { precision }[0 ; 1]=\frac{\mathrm{TP}}{\mathrm{TP}+\mathrm{FP}}
$$


Since the boundary likelihood captures the probability of a line being a 'boundary' line, a high boundary likelihood should go along with a high overlap between MCG and cadastral reference buffer:

$$
\text { overlap }[0 ; 1] \triangleq \text { boundary likelihood }[0 ; 1]
$$

Both values are not expected to be identical, which can be influenced by altering the buffer size. Our focus is on comparing RF to CNN and secondarily on the boundary likelihood itself. Results are considered only in areas for testing, in which we have cadastral reference data (Figure 7.2).

Manual vs. Automated Delineation: this analysis compares the time and number of clicks required to delineate visible boundaries once manually and once with the automated approach. Manual delineation refers to delineating parcels based on the orthoimage without further guidance. Automated delineation refers to our approach, including RF or CNN classification depending on which approach shows superior results in chapter 7.3.2. All delineations should fall within the cadastral reference buffer of $0.4 \mathrm{~m}$ radius. The buffer size represents the local accepted accuracy for cadastral delineation and falls within the $2.4 \mathrm{~m}$ proposed for rural areas by the International Association of Assessing Officers (IAAO) [303].

The comparison is conducted for a rural area in Ethiopia and two peri-urban areas in Rwanda and Kenya (Figure 7.2). No urban area is selected, as indirect surveying relies on the existence of visible boundaries, which are rare in densely populated areas [355]. Furthermore, indirect surveying in urban areas saves less logistics for field surveys, due to smaller parcel sizes. Only parcels for which all boundaries are visible and thus detectable from the orthoimage are kept for this analysis. Since no digital up-to-date cadastral reference exists for our areas in Kenya and Rwanda, cadastral reference data are created based on local knowledge in alignment with visible boundaries.

\subsection{Results}

\subsubsection{CNN Architecture}

We first tested different pre-trained base CNNs (VGG, ResNet, Inception, Xception, MobileNet, and DenseNet) to which we added trainable layers. The combined CNN model was trained with a batch size of 32 for 100 epochs. In the case of no learning, the training stopped earlier. We used $10 \%$ of the balanced training data for validation. These data were not seen by the network, but used only to calculate loss and accuracy per epoch. These metrics and their curves looked most promising for VGG19 [349]. VGG19 is a 19 layer deep CNN developed by the Visual Geometry Group (VGG) from University of Oxford. VGG19 is trained to classify images into 1000 object categories, such as 
keyboard, mouse pencil, and many animals. The network has learned highlevel features for a wide range of images from I mageNet [356]. I mageNet, is a dataset of over 15 million labelled high-resolution images with around 22,000 categories. Compared to other CNNs, VGG has shown to generalize well compared to more complex and less deep CNN architectures [349].

We used VGG19 layers pre-trained for 20,024,384 parameters as a base model. Next, we modified hyper-parameters for VGG19 on the learning optimizer, the depth of the fully connected layer, and the dropout rate to optimize accuracy and loss. We used softmax as an activation function to retrieve predictions for tiles being 'not boundary' in the range [0; 1$]$. These values represent the weights for the later least-cost-path calculation. Sigmoid activation, which is a type of softmax for a binary classification problem, provided similar results in terms of accuracy and loss. However, it required more post-processing, as the resulting value in the range $[0 ; 1]$ cannot be understood as described for softmax activation.

The aim was to maximize the accuracy for training and validation data, while minimizing loss. To avoid over-fitting, the curves for training and validation accuracy should not diverge, which was achieved by increasing the dropout rate from 0.5 to 0.8 . To avoid under-fitting, the curve for training accuracy should not be below that of validation accuracy, which was avoided by increasing the depth of the fully connected layer from 16 to 1024 . To avoid oscillations in loss, the learning rate was lowered from 0.01 to 0.001 . Learning was stopped once the validation accuracy did not further improve. Results and observations derived from different hyper-parameter settings and different pre-trained base CNNs are provided in the appendix.

We achieved the best results after training 8,242 parameters on four trainable layers added to 22 pre-trained VGG19 layers (Table 7.3). This led to a validation accuracy of $71 \%$ and a validation loss of 0.598 after 200 epochs (Figure 7.8). The accuracy could be increased by $1 \%$ after 300 epochs, with validation loss restarting to increase to 0.623 . We conclude that optimal results are achieved after 200 epochs. 100 epochs halve the training time to 11 hours, while obtaining $1 \%$ less accuracy and a loss of 0.588 . The implementation relies on the open source library Keras [357] and is publically available [305]. All experiments are conducted on a machine having a NVIDIA GM200 (GeForce GTX TITAN X) GPU with 128 GB RAM. 
Table 7.3. Settings for our fine-tuned CNN based on VGG19.

\begin{tabular}{rll}
\hline $\begin{array}{r}\text { untrainable } \\
\text { layers }\end{array}$ & Settings & Parameters \\
\hline \multirow{2}{*}{$\begin{array}{r}\text { trainable } \\
\text { layers }\end{array}$} & pooling layer & $\begin{array}{l}\text { exclusion of final pooling and fully } \\
\text { connected layer }\end{array}$ \\
\cline { 2 - 3 } & fully connected layer & global average pooling 2D \\
\cline { 2 - 3 } & dropout layer & depth=1024, activation=ReLu \\
\cline { 2 - 3 } $\begin{aligned} \text { learning } \\
\text { optimizer }\end{aligned}$ & $\begin{array}{l}\text { stochastic gradient } \\
\text { descent (SGD) optimizer }\end{array}$ & $\begin{array}{l}\text { learning rate }=0.001 \\
\text { momentum }=0.9 \\
\text { decay=learning rate/epochs }\end{array}$ \\
\hline \multirow{2}{*}{ training } & $\begin{array}{l}\text { shuffled training tiles and } \\
\text { un-shuffled validation tiles }\end{array}$ & $\begin{array}{l}\text { epochs }=\text { max. } 200 \\
\text { batch size }=32\end{array}$ \\
\hline
\end{tabular}

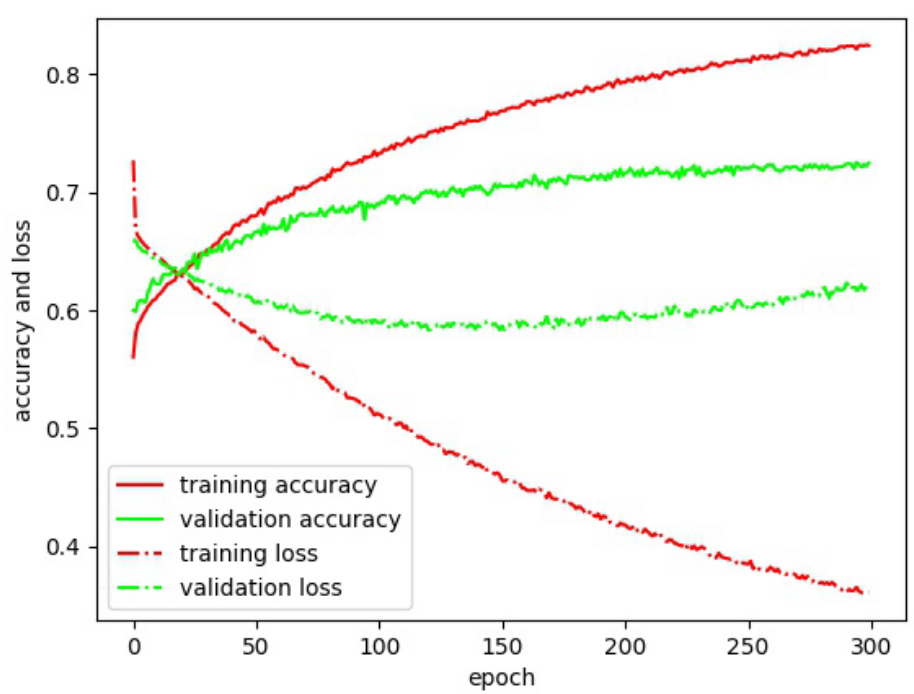

Figure 7.8. Accuracy and loss for our fine-tuned VGG19.

\subsubsection{RF vs. CNN Classification}

Of those lines that should get a boundary likelihood $>0$, i.e., those that fall within the cadastral reference buffer, $100 \%$ for RF and $98 \%$ for CNN are assigned a boundary likelihood $>0$ (Table 7.4). This means that both classifiers predict a boundary likelihood in the range 10; 1 ] when there is some overlap with the cadastral reference buffer.

Next, we looked at how valid the boundary likelihood is, i.e., whether its value is equal to the line's overlap with the cadastral reference buffer. For this, we excluded lines having no overlap with the cadastral reference buffer, i.e., those having an overlap $=0$. We grouped the remaining lines to compare boundary likelihood and overlap values (Table 7.5). For RF-derived boundary likelihoods, we obtained an accuracy of $41 \%$ and a precision of $49 \%$. For CNNderived boundary likelihoods, we obtained an accuracy of $52 \%$ and a precision 
of $76 \%$. The percentage of lines per value interval of 0.25 for the same boundary likelihood and overlap value deviated on average by $15 \%$ for RF and by $7 \%$ for CNN (Table 7.5).

Overall, CNN-derived boundary likelihoods obtained a similar recall, a higher accuracy, and a higher precision (Table 7.4). The percentage of lines for different ranges of boundary likelihoods represented the distribution of overlap values more accurately (Table 7.5). Even though the values of overlap and boundary likelihood do not express the same, they provide a valid comparison between RF- and CNN-derived boundary likelihoods. We consider CNN-derived boundary likelihoods a better input for the interactive delineation and continue the accuracy assessment for a boundary classification based on CNN.

Table 7.4. Is the boundary likelihood predicted for the correct lines?

\begin{tabular}{|c|c|c|c|c|c|c|}
\hline \multicolumn{7}{|c|}{ overlap } \\
\hline \multirow{5}{*}{ 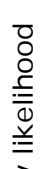 } & & & 0 & ]0; 1] & $\Sigma$ & $\Sigma \%$ \\
\hline & \multirow{4}{*}{ RF } & 0 & 535 & 265 & 800 & 0 \\
\hline & & ]0; 1] & 150,583 & 59,123 & 209,706 & 100 \\
\hline & & $\Sigma$ & 151,118 & 59,388 & 210,506 & \\
\hline & & $\sum \%$ & 72 & 28 & & 100 \\
\hline \multirow{4}{*}{$\begin{array}{l}\frac{1}{1} \\
\frac{\pi}{0} \\
\subseteq \\
0 \\
0 \\
0\end{array}$} & \multirow{4}{*}{ CNN } & 0 & 7,560 & 1,794 & 9,354 & 4 \\
\hline & & ]0; 1] & 145,558 & 57,594 & 201,152 & 96 \\
\hline & & $\Sigma$ & 151,118 & 59,388 & 210,506 & \\
\hline & & $\Sigma \%$ & 72 & 28 & & 100 \\
\hline
\end{tabular}

Table 7.5. How correct is the predicted boundary likelihood?

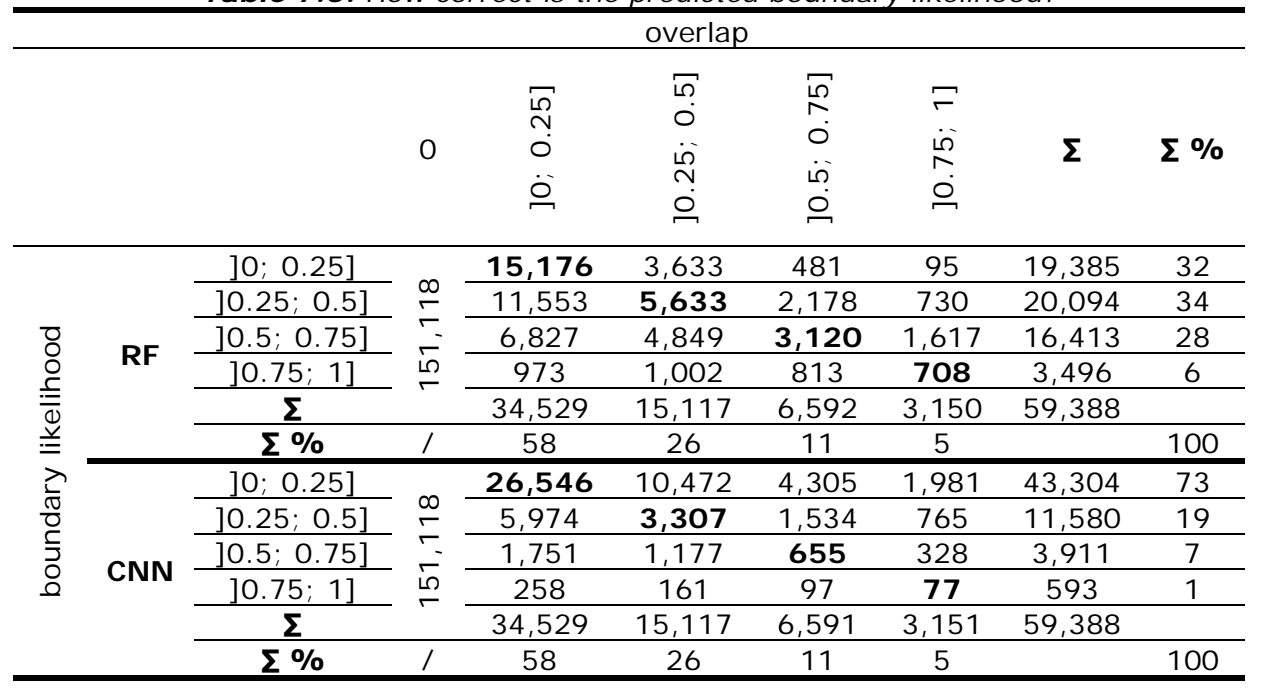

\subsubsection{Manual vs. Automated Delineation}

Before comparing manual to automated delineation, we filtered the cadastral reference data for Ethiopia (Figure 7.2b) to contain visible parcels only. We kept only those parcels for which all boundary parts were visually demarcated. 
As in [358], we consider only fully closed polygons that are entirely visible in the image. Parcels, for which all boundaries were visible amount to $38 \%$ with strong variations across the scene. In [358], this number has been reported to average around $71 \%$ for rural Ethiopian areas. We can confirm $71 \%$ for parts of our study area that cover smallholder farms. Cadastral data for Rwanda and Kenya were delineated based on local knowledge in alignment with visible boundaries. As for Ethiopia, only fully closed and visible parcels were considered. The mean size of our visible parcels amounts to $2,725 \mathrm{~m}^{2}$ for Ethiopia, $656 \mathrm{~m}^{2}$ for Rwanda, and $730 \mathrm{~m}^{2}$ for Kenya.

When manually delineating visible boundaries, we observed how tiring a task as manual delineation is: the delineator has to continuously scan the image for visible boundaries to then click precisely and repeatedly along the boundary to be delineated. Apart from the visual observation of the orthoimage, the delineator has no further guidance on where to click. Each parcel is delineated the same way, which makes it a highly repetitive task that exhausts eyes and fingers in no time.

When comparing manual to automated delineation, this impression changes: the delineator now has lines and vertices to choose from, which can be connected automatically using multiple functionalities (Table 7.2, Figure 7.6). Complex, as well as simple parcels, require fewer clicking when delineating with the automated approach: to follow a curved outline, manual delineation requires frequent and accurate clicking while zooming in and out. Automated delineation requires clicking on vertices covering the start and endpoint once before they are automatically connected precisely following object outlines (Figure 7.6d). Similarly, the automated delineation is superior for simple rectangular parcels: while manual delineation requires accurate clicking on each of the at least four corners of a rectangle, automated delineation allows clicking once somewhere inside the rectangle to retrieve its outline (Figure 7.9a).

However, choosing the optimal functionality can be time-consuming, especially in cases of fragmented MCG lines obtained from high-resolution UAV data. We assume that the time for automated delineation can be reduced through increased familiarity with all functionalities and by further developing their usability, e.g., by keyboard shortcuts.

Automated delineation required fewer clicks for our rural and peri-urban study areas (Table 7.6). Only those parcels for which one of our functionalities was more effective than manual delineation are considered for the automated delineation, amounting to $40-58 \%$ of all visible parcels. The effectiveness of manual delineation is considered for all $100 \%$ of the visible parcels. By maximizing the number of delineated parcels, we aimed to minimize the effect 
of unusual parcels that required much effort to delineate manually. We expect the measures that we obtained for the manual delineation to be similar for the $40-58 \%$ of parcels considered for the automated delineation. For the remaining parcels, MCG lines were either not available, or not aligning enough with the reference data. Manually delineating these parcels with the plugin requires the same number of clicks and time as conventional manual delineation, but is partly less tiring, as the delineation can be snapped to the MCG lines and vertices.

Nevertheless, the lines and vertices can also impede the visibility: for our data from Rwanda and Kenya, the boundaries are not continuously visible. The partly vegetation-covered boundaries result in zigzagged and fragmented MCG lines (Figure 7.9b). Additionally, visible boundaries with low contrast were partly missed by MCG image segmentation. In both cases, the advantages of automated delineation are limited.

In chapter 6 and 7, we claimed that the least-cost-path based on the boundary likelihood is beneficial to delineate long and curved outlines [300]. For the Ethiopian data, we now rarely made use of the boundary likelihood: for the often small and rectangular parcels, connecting all lines surrounding a click or a selection of lines was more efficient. For areas with few fragmented, long or curved outlines, the workflow is assumed to be of similar effectiveness when leaving out the boundary classification. To include the boundary classification is beneficial when boundaries are demarcated, e.g., by long and curved roads, visibly confined waterbodies, or circular vegetation as demonstrated for Kajiado in chapter 7.

For our data from Kenya and Rwanda, we omitted the boundary classification, since we hardly used it for the Ethiopian data. The least-cost-path, for which a weight attribute can be selected in the plugin interface, used line length instead of boundary likelihood. Since the boundaries differ from the boundaries in the Ethiopian scene, the CNN would need to be retrained or fine-tuned for the new boundary types. Retrieving CNN-derived boundary likelihoods for these UAV data, would require further experiments on whether and how to rescale tiles to $224 \times 224$ pixels while providing context comparable to our aerial tiles (Figure 7.5).

Overall, the automated delineation provided diverse functionalities for different boundary types (Table 7.7), which made delineation less tiring and more effective (Table 7.6). Improvements to manual delineation were strongest for parcels fully surrounded by MCG lines. Such parcels were mostly found in the Ethiopian rural scene, where boundaries aligned with agricultural fields. In the Rwandan scene, automated delineation was time-consuming, since the boundaries were not demarcated consistently. Selecting and joining 
fragmented MCG lines required more careful visual inspection compared to the rural Ethiopian scene. In the Kenyan scene, the boundaries were less often covered by vegetation and in general better visible. Compared to the rural Ethiopian scene, the automated delineation still required more zooming, as boundaries were demarcated by more diverse objects.

Table 7.6. Does automated delineation cost less effort?

\begin{tabular}{|c|c|c|c|c|c|c|}
\hline & \multicolumn{3}{|c|}{ manual delineation } & \multicolumn{3}{|c|}{ automated delineation } \\
\hline & $\begin{array}{l}\text { parcel } \\
\text { count }\end{array}$ & $\begin{array}{c}\frac{\text { time }}{\text { parcel }} \\
{[\mathrm{s}]}\end{array}$ & $\frac{\text { clicks }}{\text { parcel }}$ & $\begin{array}{l}\text { parcel } \\
\text { count }\end{array}$ & $\begin{array}{c}\frac{\text { time }}{\text { parcel }} \\
{[\mathrm{s}]}\end{array}$ & $\frac{\text { clicks }}{\text { parcel }}$ \\
\hline Ethiopia (rural) & 350 & 13 & 10 & $181(52 \%)$ & 8 & 2 \\
\hline Rwanda (peri-urban) & 100 & 12 & 7 & $40(40 \%)$ & 25 & 5 \\
\hline Kenya (peri-urban) & 272 & 11 & 5 & $157(58 \%)$ & 10 & 4 \\
\hline
\end{tabular}



(a)

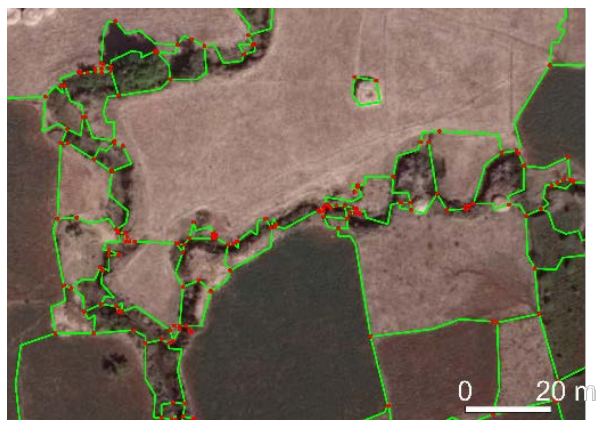

(b)

Figure 7.9. (a) Automated delineation requires clicking once somewhere in the parcel, while manual delineation requires precise clicking at least four times on each corner. (b) Boundaries partly covered or delineated by vegetation impede indirect surveying and limit the effectiveness of our automated delineation compared to manual delineation.

Table 7.7. Which plugin functionality to use for which boundary type?

\begin{tabular}{|c|c|c|c|}
\hline Functionality & Boundary type & $\begin{array}{c}\text { Boundary } \hat{=} \\
\text { Segmentation }\end{array}$ & $\begin{array}{l}\text { Example } \\
\text { boundary }\end{array}$ \\
\hline $\begin{array}{r}\text { Connect around } \\
\text { selection }\end{array}$ & $\begin{array}{l}\text { complex or } \\
\text { rectangular }\end{array}$ & yes & agricultural field \\
\hline $\begin{array}{r}\text { Connect lines' } \\
\text { endpoints }\end{array}$ & $\begin{array}{l}\text { small or } \\
\text { rectangular }\end{array}$ & partly & vegetation-covered \\
\hline $\begin{array}{r}\text { Connect along } \\
\text { optimal path }\end{array}$ & long or curved & yes & curved river \\
\hline $\begin{array}{r}\text { Connect manual } \\
\text { clicks }\end{array}$ & $\begin{array}{l}\text { fragmented or } \\
\text { partly invisible }\end{array}$ & no or partly & low-contrast \\
\hline
\end{tabular}

\subsection{Discussion}

How we reformulated our problem to be solvable by a tile-based CNN has been similarly proposed in biomedical optics [359]. Fang et al. crop tiles centered on retinal boundary pixels and train a CNN to predict nine different boundary labels. Correspondingly labelled pixels are connected with a graph-based 
approach. To transfer the latter to our case, we may investigate whether connecting tiles of similar boundary likelihood can omit the need for an initial MCG image segmentation: by using Fully Convolutional Networks (FCN) [360] each pixel of the input image would be assigned a boundary likelihood, which can be connected using Ultrametric Contour Maps (UCM) [249] included in MCG as investigated in [334]. Connecting pixels of corresponding boundary likelihoods could also be realized by using MCG-based contour closure [361], line integral convolution [362], or template matching [363].

Alternatively, the topology of MCG lines can be used to sort out false boundary likelihoods before aggregating them per line: this could be realized by not shuffling training data and thus maintaining more context information per batch, or by using graph-based approaches such as active contour models [191] suggested for road detection [364,365], or region-growing models suggested for RF-based identification of linear vegetation [366].

Predicting the optimal MCG parameter k per image may also be achieved with CNNs. Depending on whether an area is, e.g., rural or urban, cadastral parcels vary in size and shape. Larger parcels demand less over-segmentation and a higher k. Similarly, our high-resolution UAV data required a higher k, i.e., 0.3 and 0.4 as compared to 0.1 for the aerial data. Challenges to be addressed are training with data from multiple sensors, varying parcel sizes in training, and automatically labelling data with the optimal segmentation parameter $\mathrm{k}$.

When training a network to predict boundary likelihoods for visible object outlines, our training data based on cadastral reference are beneficial as it is available without further processing. The data have little bias, as no human annotator with domain knowledge is required [367]. However, the data could be improved: cadastral data contain invisible boundaries not detectable by MCG. To limit training data to visible boundaries would match better with what the network is expected to learn and increase achievable accuracy metrics.

When deciding whether to use RF or CNN for boundary classification, one needs to balance feature extraction for RF [368] against training data preparation and computational requirements for CNN [335]. In cases of limited training data for CNN, our CNN-based boundary classification may be adopted by data augmentation and re-balancing class weights. One advantage of our RF-based boundary classification is that it contains a feature capturing 3D information from a Digital Surface Model (DSM), which we used in [300]. 3D information still needs to be included in the CNN-based boundary classification.

Compared to computer vision, the amount and size of benchmark image data are marginal: existing benchmarks cover aerial data for urban object classification [369] and building extraction [333], satellite imagery for road 
extraction, building extraction and land cover classification [370], as well as satellite and aerial imagery for road extraction [371]. Such benchmarks in combination with open data initiatives for governmental cadastral data [337], aerial imagery [339], and crowdsourced labelling [372-374] may propel deep learning frameworks for cadastral boundary delineation, i.e., cadastral intelligence. Instead of using a VGG pre-trained on ImageNet, our approach could then be trained on diverse remote sensing and cadastral data, resulting in a possibly more effective and scalable network.

\subsection{Conclusion}

By reformulating our problem to be solvable by a CNN, we have investigated integrating a more state-of-the-art approach in our previously proposed boundary delineation workflow [300]. Our workflow consists of image segmentation, boundary classification, and interactive delineation: for boundary classification, a deep learning CNN was assumed to be superior to machine learning RF, as CNNs require no hand-crafted features and can be trained incrementally.

This starting hypothesis held true: even though pre-trained on images from computer vision, transfer-learning a CNN on remote sensing data provided more accurate predictions for boundary likelihoods compared to RF. Our successful integration reduces the effect of possibly meaningless or biased hand-crafted features and increases the degree of automation of our approach. However, when conducting the final workflow step, i.e., interactive delineation, we found that we seldom made use of the boundary likelihood. We reduced over-segmentation, due to post-processing the image segmentation. This, in combination with new interactive delineation functionalities, is more effective than manual delineation for regular-shaped parcels surrounded by visible boundaries. The delineation functionality that uses boundary likelihood is beneficial for long or curved boundaries, which was rare in our study areas.

Our approach is now more automated due to the integration of deep learning and has proven to be less tiring and more effective compared to manual delineation due to the decreased over-segmentation and our new delineation functionalities. The approach works on data from different sensors (aerial and UAV) of different resolutions (0.02-0.25 m). Advantages are strongest when delineating in rural areas due to the continuous visibility of monotonic boundaries.

Despite these advances, automating cadastral boundary delineation is not at its end. Identifying areas in which a large portion of cadastral boundaries is visible and for which high-resolution remote sensing and up-to-date cadastral data are available in digital form still impedes methodological development. 
Future work could investigate the approach's applicability for invisible boundaries, that are marked before UAV data capture, e.g., with paint or other temporary boundary markers. In this context, the degree to which the approach can support participatory mapping could also be investigated. Furthermore, research needs to be done on how to align innovative approaches with existing technical, social, legal, and institutional frameworks in land administration as addressed in [348,375,376]. We are pursuing this by providing documentation and testing material [377] that enables surveyors and policy makers in land administration to easily understand, test, and adapt our approach. 


\subsection{Appendix}

Table 7.8. Results obtained on validation data for different fine-tuned CNNs.

The one used for further analysis in our study is outlined in green. The legend text corresponds to that of Figure 7.8.

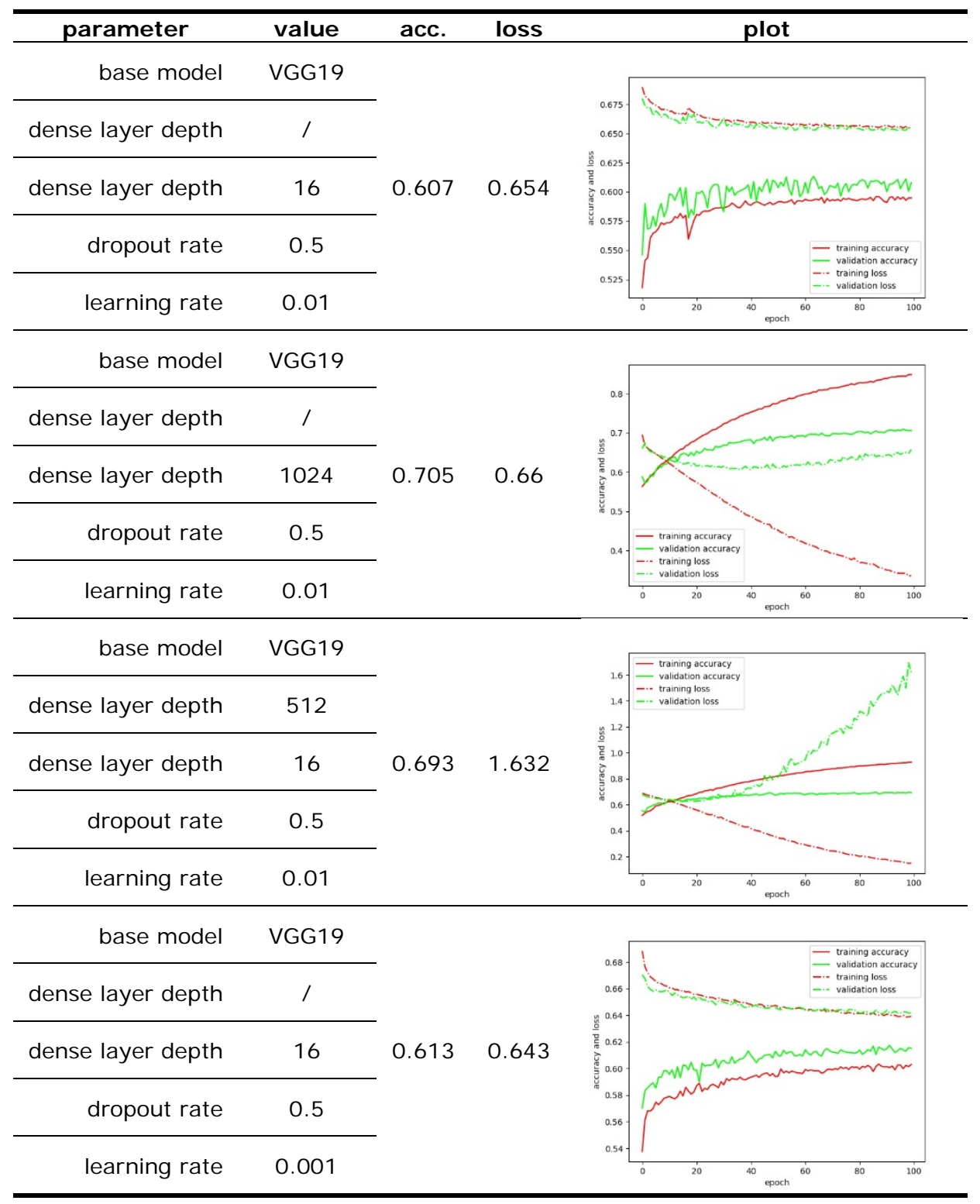




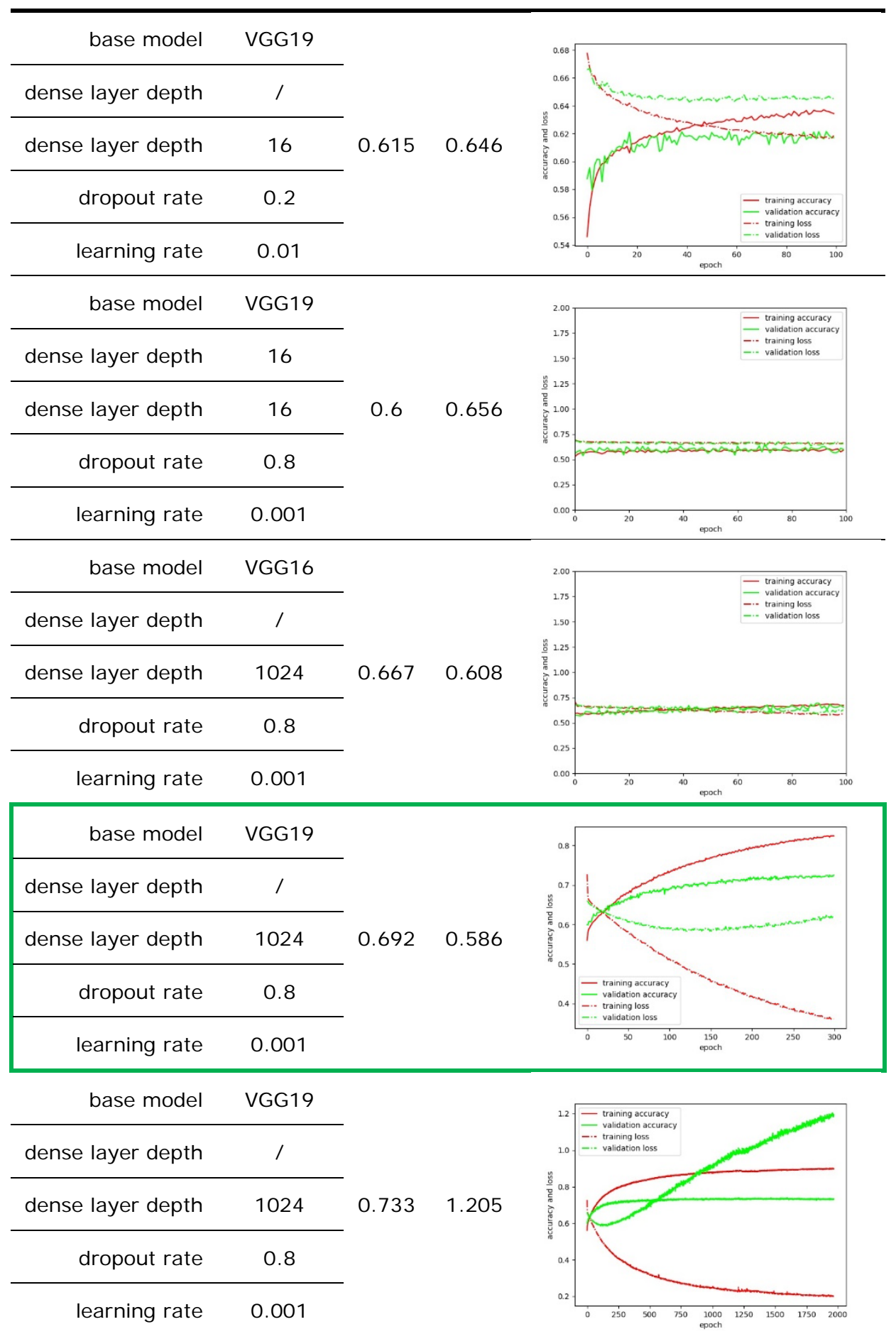









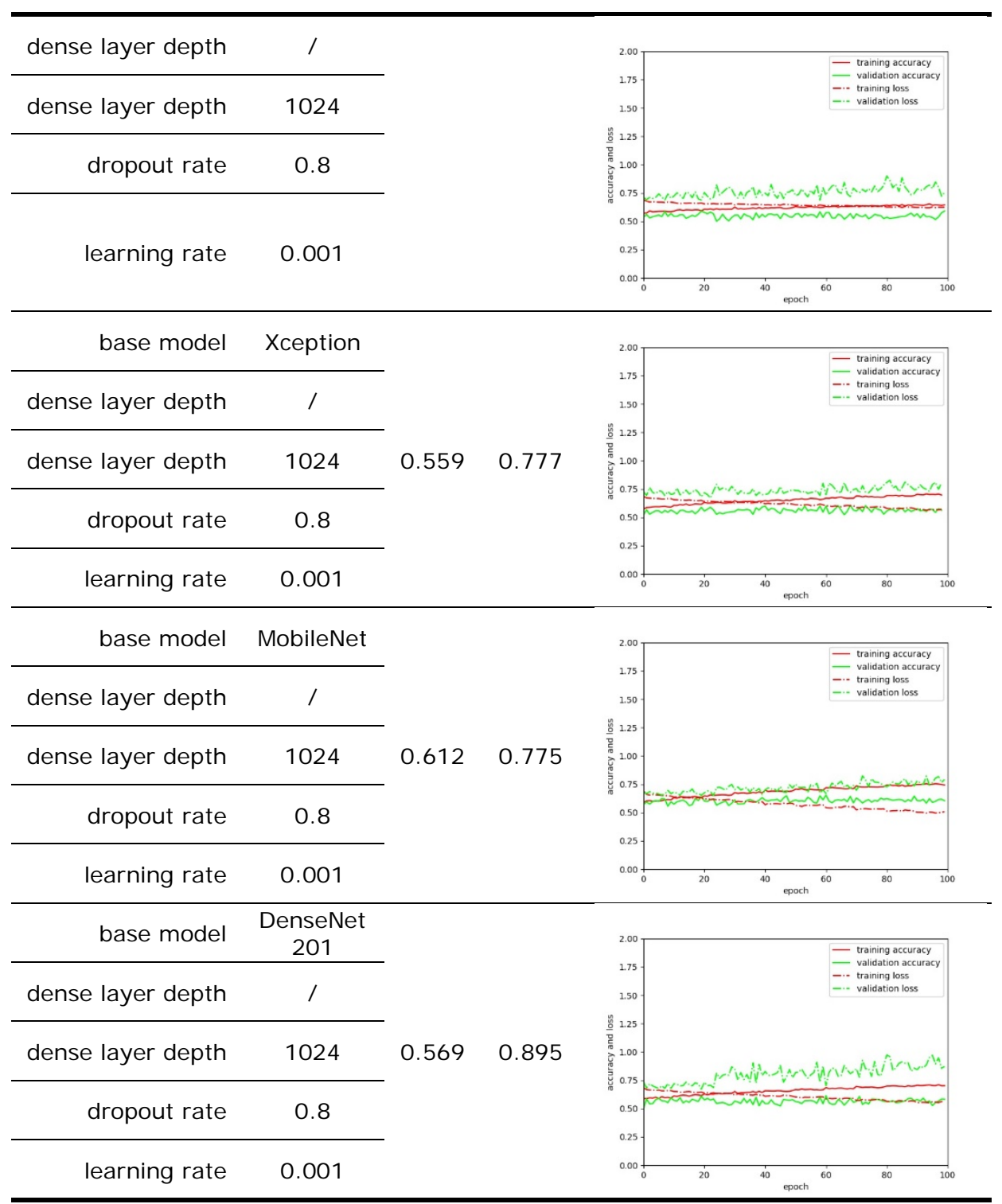




\section{Synthesis ${ }^{*}$}

* This chapter is based on the previous chapters, including lessons learned in
(co-)authoring the following publications: 3. Koeva, M.; Crommelinck, S.; Stöcker, C.; Crompvoets, J.; Ho, S.; Buntix, I.; Schwering, A.; Chipofya, M.; Sahib, J.; Zein, T., et al. In Its4land Challenges and Opportunities in Developing Innovative Geospatial Tools for Fitfor-Purpose Land Rights Mapping, FIG Congress, Istanbul, Turkey, 6-11 May, 2018; pp 1-17.

4. Bennett, R.; Gerke, M.; Crompvoets, J.; Ho, S.; Schwering, A.; Chipofya, M.; Schultz, C.; Zein, T.; Biraro, M.; Alemie, B., et al. In Building Third Generation Land Tools: Its4land, Smart Sketchmaps, UAVs, Automatic Feature Extraction, and the Geocloud, World Bank Conference on Land and Poverty, Washington, DC, USA, 20-24 March, 2017; pp 1-23.

378. Koeva, M.; Bennett, R.; Gerke, M.; Crommelinck, S.; Stöcker, C.; Crompvoets, J.; S.Ho; Schwering, A.; Chipofya, M.; Schultz, C., et al. In Towards I nnovative Geospatial Tools for Fit-for-Purpose Land Rights Mapping, ISPRS Geospatial Week, Wuhan, China, 18-22 September, 2017; XLII-2/W, pp 37-43.

379. Gerke, M.; Stöcker, C.; Crommelinck, S.; Koeva, M. UAV Für Das Kadaster - Das Eu-Projekt Its4land. In UAV 2018 - Vermessung Mit Unbemannten Flugsystemen: Beiträge Zum 169. Dvw-Seminar, Deutsche Gesellschaft fur Geodesie, Geoinformation, und Landmanagement (DVW): 2018; Vol. 89, pp 105-116.

380. Kohli, D.; Crommelinck, S.; Bennett, R.; Koeva, M.; Lemmen, C. In Object-Based I mage Analysis Methods for Cadastral Boundary Mapping Using Satellite Images, Image and Signal Processing for Remote Sensing XXIII, Warsaw, Poland, 4 October 2017, 2017; 10427.

381. Crommelinck, S.; Koeva, M. Towards Cadastral Intelligence? GIM International 2019, 27-29.

382. Marshall, M.; Crommelinck, S.; Kohli, D.; Perger, C.; Yang, M.Y.; Ghosh, A.; Fritz, S.; Bie, K.d.; Nelson, A. Crowd-Driven and Automated Mapping of Field Boundaries in Highly Fragmented Agricultural Landscapes of Ethiopia with Very High Spatial Resolution Imagery. Remote Sensing 2019, 11, 2082. 
The main goal of this Ph.D. research was to develop an approach that simplifies image-based cadastral mapping to support the automated mapping of land tenure. The goal has been pursued by developing an automated cadastral boundary delineation approach applicable to UAV imagery and further sources of remote sensing data.

Overall, this manuscript illustrates how state-of-the-art knowledge from remote sensing, geo-informatics, photogrammetry, computer vision, and machine learning can be combined into an innovative cadastral mapping approach. By making use of synergies, we developed an approach that is superior to manual delineation when object outlines are continuously visible and coincide with cadastral boundaries. In future work, it can be adapted and transferred to real-world cadastral mapping use cases. The automated approach simplifies and speeds-up the delineation of objects from imagery. While the approach has been developed for cadastral mapping, it can also be used to delineate objects in other application fields, such as land use mapping, topographical mapping, road tracking, or building extraction. This Ph.D. research can be considered an innovative impulse for improving manual delineation in land administration and beyond.

\subsection{Conclusions per Objective}

\section{(i) To review relevant information}

This objective is addressed in chapters 1 and 3 . The extensive literature review provided in chapter 3 deals with concepts and case studies related to this Ph.D. research. We review the state-of-the-art on cadastral mapping, boundary delineation, UAV photogrammetry, feature extraction, as well as their interactions. The review reveals that automating indirect surveying from UAV data is a recently emerging research field. In practice, indirect surveying from UAV or any other remotely sensed imagery appears to be rarely automated and relies mostly on manual delineation through on-screen delineation.

Reviewing background information helped to contextualize later results of this Ph.D. research, to raise awareness for bottlenecks and lessons learned in related studies, and to depict the potential of automated UAV-based cadastral mapping. Most important for subsequent work was the knowledge gained on feature extraction methods synthesized in a workflow for automated UAVbased cadastral mapping. This knowledge served as a basis for the development of a corresponding workflow. Similarly, the knowledge gained on accuracy assessment helped in the later design of appropriate evaluation measures. 


\section{(ii) To develop a suitable approach}

This objective is addressed in chapters 4, 5 and 6 . This includes developing and testing the workflow synthesized from the review in chapter 3. The workflow consists of image segmentation, line extraction and contour generation. These steps are addressed one after another in chapters 4, 5 and 6 , respectively.

We observe that some of the workflow steps, such as image segmentation and line extraction, are solved more efficiently in the computer vision community compared to the remote sensing one. Transferring suitable approaches to remote sensing and testing their applicability for UAV-based cadastral mapping proved beneficial.

In contrast to our initial assumption that the entire workflow could be implemented in an open-source GIS, we realized that state-of-the-art approaches are often available only as a standalone solution for a specific software, on a specific operating system, and in a specific programming language. It became necessary to develop the workflow in a modular fashion that integrates different source code implementations.

From presenting at conferences and discussing on-going work, it became evident that an automatic extraction of boundaries is only part of the solution: even after successful development of an approach that extracts $99 \%$ of all visible boundaries, the approach might not reach acceptance and applicability. A surveyor would have to check all results to find the missing $1 \%$. Defining land property rights is too sensitive a task to be left completely to an algorithm.

We stepped back from the idea of automatically generating boundaries that require some post-editing before obtaining final cadastral boundaries. Instead, we shifted to the idea of automatically extracting boundary features that support an interactive delineation before obtaining final cadastral boundaries. It became evident that providing a solution for what we considered first as 'some post-editing' and what we then implemented as an interactive delineation is of major importance for the acceptance and applicability of automated cadastral mapping. Bridging the gap between an automatically extracted result and the desired end product should not be neglected and should receive more attention in research. 


\section{(iii) To optimize and evaluate the developed approach}

This objective is addressed in chapters 7 and 8 . We investigate how the approach can be transferred further from ideation to application. In chapter 7 , the automated part is altered once more, by combining the steps of image segmentation and line extraction and thus reducing the workflow's complexity. The cycle of designing, developing, testing and evaluating the workflow or parts of it has been repeated several times in the course of this Ph.D. research. Chapter 7 takes a step back and addresses a more global picture, in which potential end users are involved and asked for feedback. This contributed in evaluating the interactive delineation, before being optimized in the following chapter. In chapter 8 , the machine learning part of the workflow is improved by replacing hand-crafted features and RF by deep learning CNNs. In the end, the newly added functionalities for the interactive delineation make our approach more effective compared to manual delineation even without CNNderived boundary likelihoods.

While our previous evaluations use objects such as roads and buildings potentially demarcating cadastral boundaries, evaluations in chapter 7 and 8 compare results to the intended end product: cadastral boundaries. Overall, the analysis reveals that successful application of the approach requires more cycles of designing, developing, testing and evaluating. The questions and schemas developed within this Ph.D. research can contribute to creating and implementing corresponding cycles to mature the approach's technological readiness level. As part of its4land, we are developing documentation and testing material that enables developers, surveyors and policy makers in land administration to easily understand, test and adapt our approach.

\subsection{Reflections and Outlook}

This work was motivated by the large number of people without access to formal land administration. The lack of recorded land rights creates insecure land tenure and existence-threatening conflicts. This research aims to support the recording of land rights by simplifying cadastral mapping, which is considered the most expensive part of the land administration system [7]. The developed approach is inspired by geo-technology taking into account stateof-the-art knowledge from remote sensing, geo-informatics, photogrammetry, computer vision, and machine learning.

Discussing the work at conferences of these different communities, as well as in the frame of the inter-disciplinary its4land project, raised the awareness of different problem perceptions and thus different approaches to solution development. Integrating these different perspectives was beneficial and necessary. On the one hand, cadastral boundary extraction can be considered a problem of image segmentation since a boundary is not necessarily an object, 
but the separation line between multiple objects. On the other hand, it can be considered a classification problem since a boundary can be demarcated by an object. Similarly, high-resolution data obtained through a remote sensing platform, such as an UAV, might be better analyzed through a computer vision approach typically designed for high-resolution data. However, computer vision approaches might not account for different characteristics of remote sensing data. Another example is machine learning. Research on machine learning focusses on how to solve complex problems in an efficient and accurate way. However, it remains crucial for a latter acceptance and applicability to adapt understanding and functioning of an approach to a specific application domain. This is seldom addressed in machine learning research. Similarly, the highresolution of UAV-based imagery might allow the extraction of visible boundaries with high spatial accuracy. However, current regulatory frameworks on UAV data capture can turn the use of other data sources such as aircraft or satellite imagery into a faster, cheaper, and more applicable choice.

Throughout this Ph.D. research, we had to find the balance between demands and expectations from different perspectives. Developing an approach that is of contribution to different scientific communities and to potential end-users demanded multiple cycles of designing, developing, testing and evaluating the approach with altering foci. At the beginning, we focused on an accurate object extraction. Later, our focus shifted to an effective and applicable mapping solution.

At the beginning of this Ph.D. research, we found that automating indirect surveying from UAV data was a recently emerging research field. During the past 3.5 years, a growing interest has been manifested, e.g., in a symposium on feature extraction for cadastral boundaries and several master students at the Faculty of Geo-information Science and Earth Observation of the University of Twente worked on related method development and applicability. In the African context, we observed more projects funded by the World Bank on automatically mapping, e.g., smallholder rights, and conferences aiming at digitizing land administration by strengthening ICT-related research. Nevertheless, automating indirect surveying from UAV data remains rarely investigated in depth outside of this Ph.D. research.

The initial goal proposed in its4land was to fully automate the extraction of cadastral boundaries: we aimed to translate the intelligence of a human delineator into a machine learning approach. So far, our workflow based on deep learning has not been shown 'intelligent' enough to replace a human delineator. Even with an optimally predicted boundary likelihood, a human is still required to select which lines to convert to boundaries. Ideally, deep learning methods would learn and apply this by themselves. Cadastral 
intelligence, which we define as deep learning frameworks for cadastral boundary delineation, requires further improvements before replacing delineation by a human. Deep learning frameworks trained on different objects demarcating cadastral boundaries in varying scenes, as well as incremental learning from a delineation may propel cadastral intelligence. An increase in the availability of training data can be expected from the increasing number of open data initiatives. The rise of crowd-mapping platforms and participatory mapping projects might contribute valuable data to train a cadastral intelligence system. Future work should also include the protection of data privacy connected to such new forms of recording land tenure.

One major limitation throughout the Ph.D. research was the unavailability of UAV and cadastral reference data covering areas with many visible boundaries. UAV data capture in our target countries was impeded by unclear and restrictive regulations. Cadastral data were often not available in digital, largescale, and up-to-date form, which was the starting problem for this research. The lack of large datasets restricted the use of deep learning. Existing digital records did not align with visible boundaries, as they were delineated on outdated or low-resolution imagery. Boundaries were often not as visible as expected with sections being covered by vegetation or shadow. Other boundaries were not demarcated continuously. These conditions impeded method development and made us wonder whether a different approach to improving land tenure mapping might have been more fruitful.

The limited availability of UAV imagery and cadastral reference data throughout the Ph.D. research, as well as the variability in objects demarcating cadastral boundaries, made us develop a generic modular workflow. In the end, this workflow is not restricted to delineate cadastral boundaries from UAV data only. The workflow can be applied to delineate visible objects images from different sensors. Since this research relies mostly on open source libraries and frameworks, the approach is publically available and can easily be implemented and adapted.

Taking a step back, we can consider this work an innovative impulse for improving land administration. However, land administration includes more than the mapping of cadastral boundaries. A system that allows large-scale registration of unrecorded land tenure does not only have to solve spatial data acquisition and processing. A widely applicable and scalable solution that meets fit-for-purpose criteria would need to be compliant with local requirements. This demands analyses beyond accuracy and efficiency that incorporate existing social, legal, and institutional frameworks.

Taking another step back, one can evaluate how this Ph.D. research approached the nexus of methodological and applied research. This research 
demanded the development of methodological skills in image analysis, technical skills in software development, and applied skills in conducting and evaluating practical tests. For future work, we recommend to join scientists, developers, practitioners, and policy makers in a diverse team. Such a team would incorporate views, knowledge, and approaches from diverse scientific communities, practitioners, and policy makers to further develop image-based cadastral boundary mapping. 


\section{Bibliography}

1. its4land consortium. Its4land Website. Available online: https://its4land.com/ (accessed on 10 July 2019).

2. Koeva, M.; Crommelinck, S.; Stöcker, C.; Crompvoets, J.; Ho, S.; Buntinx, I.; Schwering, A.; Chipofya, M.; Jan, S.; Zein, T., et al. Its4land - Developing Innovative Geospatial Tools for Fitfor-Purpose Land Rights Mapping. Coordinates, the monthly magazine on positioning, navigation and beyond 2018, XIV, 31-38.

3. Koeva, M.; Crommelinck, S.; Stöcker, C.; Crompvoets, J.; Ho, S.; Buntix, I.; Schwering, A.; Chipofya, M.; Sahib, J.; Zein, T., et al. In Its4land - Challenges and Opportunities in Developing Innovative Geospatial Tools for Fit-for-Purpose Land Rights Mapping, FIG Congress, Istanbul, Turkey, 6-11 May, 2018; pp 1-17.

4. Bennett, R.; Gerke, M.; Crompvoets, J.; Ho, S.; Schwering, A.; Chipofya, M.; Schultz, C.; Zein, T.; Biraro, M.; Alemie, B., et al. In Building Third Generation Land Tools: Its4land, Smart Sketchmaps, UAVs, Automatic Feature Extraction, and the Geocloud, World Bank Conference on Land and Poverty, Washington, DC, USA, 20-24 March, 2017; pp 1-23.

5. United Nations. Sustainable Development Goals. Available online: https://sustainabledevelopment.un.org/sdg1 (accessed on 10 J uly 2019).

6. Enemark, S.; Bell, K.C.; Lemmen, C.; McLaren, R. Fit-forPurpose Land Administration. International Federation of Surveyors: Frederiksberg, Denmark, 2014; p 42.

7. Williamson, I.; Enemark, S.; Wallace, J.; Rajabifard, A. Land Administration for Sustainable Development. ESRI Press Academic: Redlands, CA, USA, 2010; p 472.

8. Dale, P.; McLaughlin, J. Land Administration. University Press: Oxford, 1999; p 182.

9. Zhang, C. In Development of a UAV-Based Remote Sensing System for Unpaved Road Condition Assessment, Annual Conference American Society for Photogrammetry \& Remote Sensing (ASPRS), Portland, Oregon, 28 April-2 May, 2008; pp 1-7.

10. Mumbone, M.; Bennett, R.; Gerke, M.; Volkmann, W. In Innovations in Boundary Mapping: Namibia, Customary Lands and UAVs, World Bank Conference on Land and Poverty, Washington DC, USA, 2015; pp 1-22.

11. Volkmann, W.; Barnes, G. In Virtual Surveying: Mapping and Modeling Cadastral Boundaries Using Unmanned Aerial Systems 
(UAS), FIG Congress: Engaging the Challenges - Enhancing the Relevance, Kuala Lumpur, Malaysia, 2014; pp 1-13.

12. Maurice, M.J.; Koeva, M.N.; Gerke, M.; Nex, F.; Gevaert, C. In A Photogrammetric Approach for Map Updating Using UAV in Rwanda, GeoTechRwanda, Kigali, Rwanda, 18-20 November, 2015; pp 1-8.

13. Barthel, K. Linking Land Policy, Geospatial Technology and Community Participation. Available online: http://www.globallandalliance.org/early-lessons-learned-fromtesting-uavs-for-geospatial-data-collection-and-participatorymapping/ (accessed on 10 July 2019).

14. Colomina, I.; Molina, P. Unmanned Aerial Systems for Photogrammetry and Remote Sensing: A Review. ISPRS J ournal of Photogrammetry and Remote Sensing 2014, 92, 79-97.

15. Pajares, G. Overview and Current Status of Remote Sensing Applications Based on Unmanned Aerial Vehicles (UAVs). Photogrammetric Engineering \& Remote Sensing 2015, 81, 281-329.

16. Everaerts, J. In The Use of Unmanned Aerial Vehicles (UAVs) for Remote Sensing and Mapping, ISPRS - International Archives of the Photogrammetry, Remote Sensing and Spatial Information Sciences, Beijing, China, 2008; XXXVII-B1, pp 1187-1192.

17. Remondino, F.; Barazzetti, L.; Nex, F.; Scaioni, M.; Sarazzi, D. In UAV Photogrammetry for Mapping and 3D Modeling-Current Status and Future Perspectives, ISPRS - International Archives of the Photogrammetry, Remote Sensing and Spatial Information Sciences, Zurich, Switzerland, 14-16 September, 2011; XXXVIII-1/C22, pp 25-31.

18. Watts, A.C.; Ambrosia, V.G.; Hinkley, E.A. Unmanned Aircraft Systems in Remote Sensing and Scientific Research: Classification and Considerations of Use. Remote Sensing 2012, 4, 1671-1692.

19. Yao, H.; Qin, R.; Chen, X. Unmanned Aerial Vehicle for Remote Sensing Applications-a Review. Remote Sensing 2019, 11, 1443.

20. Ali, Z.; Tuladhar, A.; Zevenbergen, J. An Integrated Approach for Updating Cadastral Maps in Pakistan Using Satellite Remote Sensing Data. International Journal of Applied Earth Observation and Geoinformation 2012, 18, 386-398.

21. Corlazzoli, M.; Fernandez, O. In Spot 5 Cadastral Validation Project in I zabal, Guatemala, ISPRS - International Archives of the Photogrammetry, Remote Sensing and Spatial Information Sciences, 2004; XXXV-7/W4, pp 291-296. 
22. Konecny, G. Cadastral Mapping with Earth Observation Technology. In Geospatial Technology for Earth Observation, Li, D.; Shan, J.; Gong, J., Eds. Springer: 2010; pp 397-409.

23. Ondulo, J.-D. In High Spatial Resolution Satellite Imagery for Pid Improvement in Kenya, FIG Congress, Munich, Germany, 813 October, 2006; pp 1-9.

24. Zevenbergen, J.; De Vries, W.; Bennett, R.M. Advances in Responsible Land Administration. CRC Press: Padstow, UK, 2015; p 279.

25. Tuladhar, A. In Spatial Cadastral Boundary Concepts and Uncertainty in Parcel-Based Information Systems, ISPRS International Archives of the Photogrammetry, Remote Sensing and Spatial Information Sciences, 1996; XXXI-3, pp 890-893.

26. Christodoulou, K.; Tsakiri-Strati, M. In Combination of Satellite Image Pan Ikonos-2 with Gps in Cadastral Applications, Workshop on Spatial Information Management for Sustainable Real Estate Market, Athens, Greece, 2002.

27. Alkan, M.; Marangoz, M. Creating Cadastral Maps in Rural and Urban Areas of Using High Resolution Satellite I magery. Applied Geo-informatics for Society and Environment 2009, 89-95.

28. Törhönen, M.P. Developing Land Administration in Cambodia. Computers, Environment and Urban Systems 2001, 25, 407 428.

29. Greenwood, F. Mapping in Practise. In Drones and Aerial Observation, New America: 2015; pp 49-55.

30. Manyoky, M.; Theiler, P.; Steudler, D.; Eisenbeiss, H. In Unmanned Aerial Vehicle in Cadastral Applications, ISPRS International Archives of the Photogrammetry, Remote Sensing and Spatial Information Sciences, Zurich, Switzerland, 2011; XXXVIII-1/C22, p 57.

31. Mesas-Carrascosa, F.J.; Notario-García, M.D.; de Larriva, J.E.M.; de la Orden, M.S.; Porras, A.G.-F. Validation of Measurements of Land Plot Area Using UAV Imagery. International Journal of Applied Earth Observation and Geoinformation 2014, 33, 270-279.

32. Rijsdijk, M.; Hinsbergh, W.H.M.V.; Witteveen, W.; Buuren, G.H.M.; Schakelaar, G.a.; Poppinga, G.; Persie, M.V.; Ladiges, R. In Unmanned Aerial Systems in the Process of Juridical Verification of Cadastral Border, ISPRS - International Archives of the Photogrammetry, Remote Sensing and Spatial Information Sciences, Rostock, Germany, 4-6 September, 2013; XL-1/W2, pp 325-331.

33. van Hinsberg, W.; Rijsdijk, M.; Witteveen, W. UAS for Cadastral Applications: Testing Suitability for Boundary Identification in Urban Areas. GIM International 2013, 27, 17-21. 
34. Cunningham, K.; Walker, G.; Stahlke, E.; Wilson, R. In Cadastral Audit and Assessments Using Unmanned Aerial Systems, Proceedings of ISPRS and UAV-g, Zurich, Switzerland, 14-16 September, 2011; pp 1-4.

35. Cramer, M.; Bovet, S.; Gültlinger, M.; Honkavaara, E.; McGill, A.; Rijsdijk, M.; Tabor, M.; Tournadre, V. In On the Use of Rpas in National Mapping-the Eurosdr Point of View, ISPRS International Archives of the Photogrammetry, Remote Sensing and Spatial Information Sciences, Rostock, Germany, 4 - 6 Septembe, 2013; Copernicus Publications: XL-1/W2, pp 93-99.

36. Haarbrink, R. In UAS for Geo-Information: Current Status and Perspectives, ISPRS - International Archives of the Photogrammetry, Remote Sensing and Spatial Information Sciences, Zurich, Switzerland, 11-16 September, 2011; XXXVIII-1/C22, pp 1-6.

37. Eyndt, T.; Volkmann, W. UAS as a Tool for Surveyors: From Tripods and Trucks to Virtual Surveying. GIM International 2013, 27, 20-25.

38. Barnes, G.; Volkmann, W. High-Resolution Mapping with Unmanned Aerial Systems. Surveying and Land Information Science 2015, 74, 5-13.

39. Nex, F.; Remondino, F. UAV for 3D Mapping Applications: A Review. Applied Geomatics 2014, 6, 1-15.

40. Heipke, C.; Woodsford, P.A.; Gerke, M. Updating Geospatial Databases from Images. In ISPRS Congress Book, Li; Chen; Baltsavias, Eds. Taylor \& Francis Group,: London, 2008; pp 355-362.

41. Jazayeri, I.; Rajabifard, A.; Kalantari, M. A Geometric and Semantic Evaluation of 3D Data Sourcing Methods for Land and Property Information. Land Use Policy 2014, 36, 219-230.

42. Zevenbergen, J.; Bennett, R. In The Visible Boundary: More Than J ust a Line between Coordinates, GeoTechRwanda, Kigali, Rwanda, 18-20 November, 2015; pp 1-4.

43. Bennett, R.; Kitchingman, A.; Leach, J. On the Nature and Utility of Natural Boundaries for Land and Marine Administration. Land Use Policy 2010, 27, 772-779.

44. Crommelinck, S.; Bennett, R.; Gerke, M.; Nex, F.; Yang, M.Y.; Vosselman, G. Review of Automatic Feature Extraction from High-Resolution Optical Sensor Data for UAV-Based Cadastral Mapping. Remote Sensing 2016, 8, 1-28.

45. Harwin, S.; Lucieer, A. Assessing the Accuracy of Georeferenced Point Clouds Produced Via Multi-View Stereopsis from Unmanned Aerial Vehicle (UAV) Imagery. Remote Sensing 2012, 4, 1573-1599. 
46. Gerke, M.; Przybilla, H.-J. Accuracy Analysis of Photogrammetric UAV I mage Blocks: Influence of Onboard RtkGnss and Cross Flight Patterns. PhotogrammetrieFernerkundung-Geoinformation 2016, 14, 17-30.

47. Tahar, K.N.; Ahmad, A. An Evaluation on Fixed Wing and MultiRotor UAV Images Using Photogrammetric Image Processing. International Journal of Computer, Electrical, Automation, Control and Information Engineering 2013, 7, 48-52.

48. Toth, C.; Jóźków, G. Remote Sensing Platforms and Sensors: A Survey. ISPRS J ournal of Photogrammetry and Remote Sensing 2016, 115, 22-36.

49. Eisenbeiss, H.; Sauerbier, M. Investigation of UAV Systems and Flight Modes for Photogrammetric Applications. The Photogrammetric Record 2011, 26, 400-421.

50. Fernández-Hernandez, J.; González-Aguilera, D.; RodríguezGonzálvez, P.; Mancera-Taboada, J. Image-Based Modelling from Unmanned Aerial Vehicle (UAV) Photogrammetry: An Effective, Low-Cost Tool for Archaeological Applications. Archaeometry 2015, 57, 128-145.

51. Zhang, C.; Kovacs, J.M. The Application of Small Unmanned Aerial Systems for Precision Agriculture: A Review. Precision Agriculture 2012, 13, 693-712.

52. Berni, J.; Zarco-Tejada, P.; Suárez, L.; González-Dugo, V.; Fereres, E. In Remote Sensing of Vegetation from UAV Platforms Using Lightweight Multispectral and Thermal Imaging Sensors, ISPRS - International Archives of the Photogrammetry, Remote Sensing and Spatial Information Sciences, 2009; XXXVIII-8, pp 1-6.

53. Puri, A.; Valavanis, K.; Kontitsis, M. In Statistical Profile Generation for Traffic Monitoring Using Real-Time UAV Based Video Data, Mediterranean Conference on Control \& Automation, Athens, Greece, 16-19 June, 2007; pp 1-6.

54. Bendea, H.; Boccardo, P.; Dequal, S.; Giulio Tonolo, F.; Marenchino, D.; Piras, M. In Low Cost UAV for Post-Disaster Assessment, ISPRS - International Archives of the Photogrammetry, Remote Sensing and Spatial Information Sciences, Beijing, China, 3-11 July, 2008; XXXVII-B8, pp 13731379.

55. Chou, T.-Y.; Yeh, M.-L.; Chen, Y.C.; Chen, Y.H. In Disaster Monitoring and Management by the Unmanned Aerial Vehicle Technology, Proceedings of the ISPRS TC VII Symposium, Vienna, Austria, 5-7 July, 2010; XXXVVII-7B, pp 137-142.

56. Irschara, A.; Kaufmann, V.; Klopschitz, M.; Bischof, H.; Leberl, F. In Towards Fully Automatic Photogrammetric Reconstruction 
Using Digital Images Taken from UAVs, Proceedings of the ISPRS TC VII Symposium, Vienna, Austria, 2010.

57. Binns, B.O.; Dale, P.F. Cadastral Surveys and Records of Rights in Land Administration. Available online: www.fao.org/docrep/006/v4860e/v4860e03.htm (accessed on 10 July 2019).

58. Alemie, B.K.; Bennett, R.M.; Zevenbergen, J. Evolving Urban Cadastres in Ethiopia: The Impacts on Urban Land Governance. Land Use Policy 2015, 42, 695-705.

59. United Nations. Land Administrtation in the UNECE Region: Development Trends and Main Principles. UNECE Information Service: Geneva, 2005; p 103.

60. Kelm, K. UAVs Revolutionise Land Administration. GIM International 2014, 28, 35-37.

61. Zevenbergen, J.; Augustinus, C.; Antonio, D.; Bennett, R. ProPoor Land Administration: Principles for Recording the Land Rights of the Underrepresented. Land Use Policy 2013, 31, 595-604.

62. Smith, B. On Drawing Lines on a Map. In Spatial Information Theory: A Theoretical Basis for GIS, Springer: 1995; pp 475484.

63. Lengoiboni, M.; Bregt, A.K.; van der Molen, P. Pastoralism within Land Administration in Kenya-the Missing Link. Land Use Policy 2010, 27, 579-588.

64. Fortin, M.-J.; Olson, R.; Ferson, S.; Iverson, L.; Hunsaker, C.; Edwards, G.; Levine, D.; Butera, K.; Klemas, V. Issues Related to the Detection of Boundaries. Landscape Ecology 2000, 15, 453-466.

65. Fortin, M.-J.; Drapeau, P. Delineation of Ecological Boundaries: Comparison of Approaches and Significance Tests. Oikos 1995, 72, 323-332.

66. Richardson, K.A.; Lissack, M.R. On the Status of Boundaries, Both Natural and Organizational: A Complex Systems Perspective. A Journal of Complexity Issues in Organizations and Management Emergence 2001, 3, 32-49.

67. Fagan, W.F.; Fortin, M.-J.; Soykan, C. Integrating Edge Detection and Dynamic Modeling in Quantitative Analyses of Ecological Boundaries. BioScience 2003, 53, 730-738.

68. Cay, T.; Corumluoglu, O.; Iscan, F. In A Study on Productivity of Satellite Images in the Planning Phase of Land Consolidation Projects, ISPRS Congress: Geo-Imagery Bridging Continents, Istanbul, Turkey, 12-23 July, 2004; pp 1-6.

69. Barnes, G.; Moyer, D.D.; Gjata, G. Evaluating the Effectiveness of Alternative Approaches to the Surveying and Mapping of 
Cadastral Parcels in Albania. Computers, Environment and Urban Systems 1994, 18, 123-131.

70. Lemmen, C.; Zevenbergen, J.A.; Lengoiboni, M.; Deininger, K.; Burns, T. In First Experiences with High Resolution Imagery Based Adjudication Approach for Social Tenure Domain Model in Ethiopia, FIG-World Bank Conference, Washington, DC, USA, March, 2009; pp 9-10.

71. Rao, S.; Sharma, J.; Rajashekar, S.; Rao, D.; Arepalli, A.; Arora, V.; Singh, R.; Kanaparthi, M. In Assessing Usefulness of High-Resolution Satellite Imagery (HRSI) for Re-Survey of Cadastral Maps, ISPRS Annals of the Photogrammetry, Remote Sensing and Spatial Information Sciences, Hyderabad, India, 912 December, 2014; II-8, pp 133-143.

72. Quackenbush, L.J . A Review of Techniques for Extracting Linear Features from Imagery. Photogrammetric Engineering \& Remote Sensing 2004, 70, 1383-1392.

73. Edwards, G.; Lowell, K.E. Modeling Uncertainty in Photointerpreted Boundaries. Photogrammetric Engineering \& Remote Sensing 1996, 62, 377-390.

74. Aien, A.; Kalantari, M.; Rajabifard, A.; Williamson, I.; Bennett, R. In Advanced Principles of 3D Cadastral Data Modelling, International Workshop on 3D Cadastres, Delft, Netherlands, 16-18 November, 2011; pp 377-396.

75. Ali, Z.; Ahmed, S. In Extracting Parcel Boundaries from Satellite Imagery for a Land Information System, International Conference on Recent Advances in Space Technologies (RAST), Istanbul, Turkey, 11-14 June, 2013; pp 79-81.

76. Lin, G.; Shen, C.; Reid, I. In Efficient Piecewise Training of Deep Structured Models for Semantic Segmentation, IEEE Conference on Computer Vision and Pattern Recognition (CVPR), Las Vegas, USA, 8-10 June, 2015; pp 1-11.

77. Hay, G.J.; Blaschke, T.; Marceau, D.J.; Bouchard, A. A Comparison of Three Image-Object Methods for the Multiscale Analysis of Landscape Structure. ISPRS Journal of Photogrammetry and Remote Sensing 2003, 57, 327-345.

78. Blaschke, T. Object Based Image Analysis for Remote Sensing. ISPRS J ournal of Photogrammetry and Remote Sensing 2010, 65, 2-16.

79. Blaschke, T.; Hay, G.J.; Kelly, M.; Lang, S.; Hofmann, P.; Addink, E.; Queiroz Feitosa, R.; van der Meer, F.; van der Werff, H.; van Coillie, F., et al. Geographic Object-Based Image Analysis - Towards a New Paradigm. ISPRS Journal of Photogrammetry and Remote Sensing 2014, 87, 180-191.

80. Benz, U.C.; Hofmann, P.; Willhauck, G.; Lingenfelder, I.; Heynen, M. Multi-Resolution, Object-Oriented Fuzzy Analysis of 
Remote Sensing Data for GIS-Ready Information. ISPRS Journal of Photogrammetry and Remote Sensing 2004, 58, 239-258.

81. Babawuro, U.; Beiji, Z. Satellite Imagery Cadastral Features Extractions Using I mage Processing Algorithms: A Viable Option for Cadastral Science. International Journal of Computer Science I ssues 2012, 9, 30-38.

82. Luhmann, T.; Robson, S.; Kyle, S.; Harley, I. Close Range Photogrammetry: Principles, Methods and Applications. Whittles: Dunbeath, UK, 2006; p 528.

83. Selvarajan, S.; Tat, C.W. In Extraction of Man-Made Features from Remote Sensing Imageries by Data Fusion Techniques, Asian Conference on Remote Sensing, Singapore, 5-9 November, 2001; 5, p 9.

84. Wang, J.; Zhang, Q. Applicability of a Gradient Profile Algorithm for Road Network Extraction-Sensor, Resolution and Background Considerations. Canadian Journal of Remote Sensing 2000, 26, 428-439.

85. Singh, P.P.; Garg, R.D. In Road Detection from Remote Sensing Images Using Impervious Surface Characteristics: Review and Implication, ISPRS - International Archives of the Photogrammetry, Remote Sensing and Spatial Information Sciences, Hyderabad, India, 9-12 December, 2014; XL-8, pp 955-959.

86. Trinder, J.C.; Wang, Y. Automatic Road Extraction from Aerial Images. Digital Signal Processing 1998, 8, 215-224.

87. Jin, H.; Feng, Y.; Li, B. In Road Network Extraction with New Vectorization and Pruning from High-Resolution Rs Images, International Conference on Image and Vision Computing (IVCNZ), Christchurch, New Zealand, 26-28 November, 2008; pp 1-6.

88. Wolf, B.-M.; Heipke, C. Automatic Extraction and Delineation of Single Trees from Remote Sensing Data. Machine Vision and Applications 2007, 18, 317-330.

89. Haralick, R.M.; Shapiro, L.G. Image Segmentation Techniques. Computer Vision, Graphics, and Image Processing 1985, 29, 100-132.

90. Pal, N.R.; Pal, S.K. A Review on Image Segmentation Techniques. Pattern Recognition 1993, 26, 1277-1294.

91. Sonka, M.; Hlavac, V.; Boyle, R. Image Processing, Analysis, and Machine Vision. Chapman \& Hal: London, 2014; p 829.

92. Burns, J.B.; Hanson, A.R.; Riseman, E.M. Extracting Straight Lines. IEEE Transactions on Pattern Analysis and Machine Intelligence 1986, PAMI-8, 425-455. 
93. Sharifi, M.; Fathy, M.; Mahmoudi, M.T. In A Classified and Comparative Study of Edge Detection Algorithms, International Conference on Information Technology: Coding and Computing, Las Vegas, USA, 8-10 April, 2002; pp 117-120.

94. Martin, D.R.; Fowlkes, C.C.; Malik, J. Learning to Detect Natural Image Boundaries Using Local Brightness, Color, and Texture Cues. IEEE Transactions on Pattern Analysis and Machine Intelligence 2004, 26, 530-549.

95. Chen, J.; Dowman, I.; Li, S.; Li, Z.; Madden, M.; Mills, J.; Paparoditis, N.; Rottensteiner, F.; Sester, M.; Toth, C., et al. Information from Imagery: ISPRS Scientific Vision and Research Agenda. ISPRS Journal of Photogrammetry and Remote Sensing 2016, 115, 3-21.

96. Nixon, M. Feature Extraction \& Image Processing. Elsevier: Oxford, 2008; p 406.

97. Petrou, M.; Petrou, C. I mage Processing: The Fundamentals. 2 ed.; J ohn Wiley \& Sons: West Sussex, UK, 2010; p 333.

98. Cheng, G.; Han, J. A Survey on Object Detection in Optical Remote Sensing I mages. ISPRS J ournal of Photogrammetry and Remote Sensing 2016, 117, 11-28.

99. Steger, C.; Ulrich, M.; Wiedemann, C. Machine Vision Algorithms and Applications. Wiley-VCH: Weinheim, Germany, 2008; p 360.

100. Karantzalos, K.; Bliziotis, D.; Karmas, A. A Scalable Geospatial Web Service for near Real-Time, High-Resolution Land Cover Mapping. IEEE Journal of Selected Topics in Applied Earth Observations and Remote Sensing 2015, 8, 4665-4674.

101. Zhang, C. Towards an Operational System for Automated Updating of Road Databases by Integration of Imagery and Geodata. ISPRS Journal of Photogrammetry and Remote Sensing 2004, 58, 166-186.

102. Heipke, C.; Pakzad, K.; Willrich, F.; Peled, A. Theme Issue: Integration of Geodata and Imagery for Automated Refinement and Update of Spatial Databases. ISPRS Journal of Photogrammetry and Remote Sensing 2004, 58, 127-128.

103. Bordes, G.; Giraudon, G.; J amet, O. Road Modeling Based on a Cartographic Database for Aerial Image Interpretation. In Semantic Modeling for the Acquisition of Topographic Information from Images and Maps, Förstner, W.; Plümer, L., Eds. Birkhäuser: Basel, Switzerland, 1997; pp 123-139.

104. Egmont-Petersen, M.; de Ridder, D.; Handels, H. Image Processing with Neural Networks-a Review. Pattern Recognition 2002, 35, 2279-2301. 
105. Buades, A.; Coll, B.; Morel, J.-M. A Review of Image Denoising Algorithms, with a New One. Multiscale Modeling \& Simulation 2005, 4, 490-530.

106. Perona, P.; Malik, J. Scale-Space and Edge Detection Using Anisotropic Diffusion. IEEE Transactions on Pattern Analysis and Machine Intelligence 1990, 12, 629-639.

107. Black, M.J.; Sapiro, G.; Marimont, D.H.; Heeger, D. Robust Anisotropic Diffusion. IEEE Transactions on Image Processing 1998, 7, 421-432.

108. Tan, D. In I mage Enhancement Based on Adaptive Median Filter and Wallis Filter, National Conference on Electrical, Electronics and Computer Engineering, Xi'an, China, 12-13 December, 2016; pp 767-772.

109. Schiewe, J. In Segmentation of High-Resolution Remotely Sensed Data-Concepts, Applications and Problems, ISPRS International Archives of the Photogrammetry, Remote Sensing and Spatial Information Sciences, Ottawa, Canada, 9-12 July, 2002; XXXIV-4, pp 380-385.

110. Dey, V.; Zhang, Y.; Zhong, M. In A Review on Image Segmentation Techniques with Remote Sensing Perspective, Proceedings of the ISPRS TC VII Symposium, Vienna, Austria, 5-7 July, 2010; XXXVIII-7a, pp 31-42.

111. Cheng, H.-D.; Jiang, X.; Sun, Y.; Wang, J. Color Image Segmentation: Advances and Prospects. Pattern Recognition 2001, 34, 2259-2281.

112. Felzenszwalb, P.F.; Huttenlocher, D.P. Efficient Graph-Based Image Segmentation. International J ournal of Computer Vision 2004, 59, 167-181.

113. Francisco, J.E.; Allan, D.J. Benchmarking Image Segmentation Algorithms. International J ournal of Computer Vision 2009, 85, 167-181.

114. Achanta, R.; Shaji, A.; Smith, K.; Lucchi, A.; Fua, P.; Susstrunk, S. SLIC Superpixels Compared to State-of-the-Art Superpixel Methods. IEEE Transactions on Pattern Analysis and Machine Intelligence 2012, 34, 2274-2282.

115. Ishida, T.; Itagaki, S.; Sasaki, Y.; Ando, H. Application of Wavelet Transform for Extracting Edges of Paddy Fields from Remotely Sensed Images. International Journal of Remote Sensing 2004, 25, 347-357.

116. Arbelaez, P.; Maire, M.; Fowlkes, C.; Malik, J. Contour Detection and Hierarchical Image Segmentation. IEEE Transactions on Pattern Analysis and Machine Intelligence 2011, 33, 898-916.

117. Pont-Tuset, J.; Arbeláez, P.; Barron, J.T.; Marques, F.; Malik, J. Multiscale Combinatorial Grouping for Image Segmentation and 
Object Proposal Generation. IEEE Transactions on Pattern Analysis and Machine Intelligence 2017, 39, 128-140.

118. Ma, L.; Cheng, L.; Li, M.; Liu, Y.; Ma, X. Training Set Size, Scale, and Features in Geographic Object-Based Image Analysis of Very High Resolution Unmanned Aerial Vehicle Imagery. ISPRS Journal of Photogrammetry and Remote Sensing 2015, 102, 14-27.

119. Liu, Y.; Li, M.; Mao, L.; Xu, F.; Huang, S. Review of Remotely Sensed Imagery Classification Patterns Based on ObjectOriented Image Analysis. Chinese Geographical Science 2006, 16, 282-288.

120. Blaschke, T.; Lang, S.; Lorup, E.; Strobl, J.; Zeil, P. ObjectOriented Image Processing in an Integrated GIS/Remote Sensing Environment and Perspectives for Environmental Applications. Environmental information for planning, politics and the public 2000, 2, 555-570.

121. Mountrakis, G.; Im, J.; Ogole, C. Support Vector Machines in Remote Sensing: A Review. ISPRS J ournal of Photogrammetry and Remote Sensing 2011, 66, 247-259.

122. Vakilian, A.A.; Vakilian, K.A. A New Satellite Image Segmentation Enhancement Technique for Weak Image Boundaries. Annals of the Faculty of Engineering Hunedoara 2012, 10, 239-243.

123. Radoux, J.; Defourny, P. A Quantitative Assessment of Boundaries in Automated Forest Stand Delineation Using Very High Resolution Imagery. Remote Sensing of Environment 2007, 110, 468-475.

124. Mueller, M.; Segl, K.; Kaufmann, H. Edge-and Region-Based Segmentation Technique for the Extraction of Large, Man-Made Objects in High-Resolution Satellite Imagery. Pattern Recognition 2004, 37, 1619-1628.

125. Wang, Y.; Li, X.; Zhang, L.; Zhang, W. In Automatic Road Extraction of Urban Area from High Spatial Resolution Remotely Sensed Imagery, ISPRS - International Archives of the Photogrammetry, Remote Sensing and Spatial Information Sciences, Beijing, China, 2008; XXXVII-B6b.

126. Kumar, M.; Singh, R.; Raju, P.; Krishnamurthy, Y. In Road Network Extraction from High Resolution Multispectral Satellite Imagery Based on Object Oriented Techniques, ISPRS Annals of the Photogrammetry, Remote Sensing and Spatial Information Sciences, Hyderabad, India, 2014; II-8, pp 107-110.

127. Butenuth, M. Segmentation of Imagery Using Network Snakes. Photogrammetrie-Fernerkundung-Geoinformation 2007, 2007, 1-7. 
128. Vetrivel, A.; Gerke, M.; Kerle, N.; Vosselman, G. In Segmentation of UAV-Based Images Incorporating 3D Point Cloud Information, ISPRS - International Archives of the Photogrammetry, Remote Sensing and Spatial Information Sciences, Munich, Germany, 25-27 March, 2015; XL-3/W2, pp 261-268.

129. Fernandez Galarreta, J.; Kerle, N.; Gerke, M. UAV-Based Urban Structural Damage Assessment Using Object-Based Image Analysis and Semantic Reasoning. Natural Hazards and Earth System Science 2015, 15, 1087-1101.

130. Grigillo, D.; Kanjir, U. In Urban Object Extraction from Digital Surface Model and Digital Aerial Images, ISPRS Annals of the Photogrammetry, Remote Sensing and Spatial Information Sciences, Melbourne, Australia, 25 August-1 September, 2012; I-3, pp 215-220.

131. Awad, M.M. A Morphological Model for Extracting Road Networks from High-Resolution Satellite Images. Journal of Engineering 2013, 1-9.

132. Ünsalan, C.; Boyer, K.L. A System to Detect Houses and Residential Street Networks in Multispectral Satellite Images. Computer Vision and Image Understanding 2005, 98, 423-461.

133. Sohn, G.; Dowman, I. In Building Extraction Using Lidar Dems and Ikonos Images, ISPRS - International Archives of the Photogrammetry, Remote Sensing and Spatial Information Sciences, Dresden, Germany, 8-10 October, 2003; XXXIV-3, pp 37-43.

134. Mena, J.B. Automatic Vectorization of Segmented Road Networks by Geometrical and Topological Analysis of High Resolution Binary Images. Knowledge-Based Systems 2006, 19, 704-718.

135. Mena, J.B.; Malpica, J.A. An Automatic Method for Road Extraction in Rural and Semi-Urban Areas Starting from High Resolution Satellite Imagery. Pattern Recognition Letters 2005, 26, 1201-1220.

136. J in, X.; Davis, C.H. An Integrated System for Automatic Road Mapping from High-Resolution Multi-Spectral Satellite Imagery by Information Fusion. Information Fusion 2005, 6, 257-273.

137. Chen, T.; Wang, J.; Zhang, K. In A Wavelet Transform Based Method for Road Extraction from High-Resolution Remotely Sensed Data, IEEE International Geoscience and Remote Sensing Symposium (IGARSS), Toronto, Canada, 24-28 June, 2002; 6, pp 3369-3371.

138. Liow, Y.-T.; Pavlidis, T. Use of Shadows for Extracting Buildings in Aerial Images. Computer Vision, Graphics, and Image Processing 1990, 49, 242-277. 
139. Liu, H.; Jezek, K. Automated Extraction of Coastline from Satellite Imagery by Integrating Canny Edge Detection and Locally Adaptive Thresholding Methods. International J ournal of Remote Sensing 2004, 25, 937-958.

140. Wiedemann, C.; Heipke, C.; Mayer, H.; Hinz, S. In Automatic Extraction and Evaluation of Road Networks from Moms-2p Imagery, ISPRS - International Archives of the Photogrammetry, Remote Sensing and Spatial Information Sciences, 1998; XXXII-3, pp 285-291.

141. Tiwari, P.S.; Pande, H.; Kumar, M.; Dadhwal, V.K. Potential of Irs P-6 Liss IV for Agriculture Field Boundary Delineation. J ournal of Applied Remote Sensing 2009, 3, 1-9.

142. Karathanassi, V.; Iossifidis, C.; Rokos, D. A Texture-Based Classification Method for Classifying Built Areas According to Their Density. International J ournal of Remote Sensing 2000, 21, 1807-1823.

143. Qiaoping, Z.; Couloigner, I. Automatic Road Change Detection and GIS Updating from High Spatial Remotely-Sensed Imagery. Geo-Spatial Information Science 2004, 7, 89-95.

144. Sharma, O.; Mioc, D.; Anton, F. In Polygon Feature Extraction from Satellite Imagery Based on Colour Image Segmentation and Medial Axis, ISPRS - International Archives of the Photogrammetry, Remote Sensing and Spatial Information Sciences, Beijing, China, 2008; XXXVII-B3a, pp 235-240.

145. Butenuth, M.; Straub, B.-M.; Heipke, C. In Automatic Extraction of Field Boundaries from Aerial Imagery, KDNet Symposium on Knowledge-Based Services for the Public Sector, Bonn, Germany, 2004; 3, pp 14-25.

146. Stoica, R.; Descombes, X.; Zerubia, J. A Gibbs Point Process for Road Extraction from Remotely Sensed Images. International J ournal of Computer Vision 2004, 57, 121-136.

147. Mokhtarzade, M.; Zoej, M.V.; Ebadi, H. In Automatic Road Extraction from High Resolution Satellite Images Using Neural Networks, Texture Analysis, Fuzzy Clustering and Genetic Algorithms, ISPRS - International Archives of the Photogrammetry, Remote Sensing and Spatial Information Sciences, Beijing, China, 2008; XXXVII-B3b, pp 549-556.

148. Zhang, C.; Baltsavias, E.; Gruen, A. Knowledge-Based Image Analysis for 3D Road Reconstruction. Asian Journal of Geoinformatics 2001, 1, 3-14.

149. Shao, Y.; Guo, B.; Hu, X.; Di, L. Application of a Fast Linear Feature Detector to Road Extraction from Remotely Sensed Imagery. IEEE Journal of Selected Topics in Applied Earth Observations and Remote Sensing 2011, 4, 626-631. 
150. Ding, X.; Kang, W.; Cui, J.; Ao, L. In Automatic Extraction of Road Network from Aerial I mages, International Symposium on Systems and Control in Aerospace and Astronautics (ISSCAA), Harbin, China, 19-21 January, 2006; pp 220-223.

151. Udomhunsakul, S.; Kozaitis, S.P.; Sritheeravirojana, U. In Semi-Automatic Road Extraction from Aerial Images, Proceedings of SPIE, Singapore, 24-27 October, 2004; International Society for Optics and Photonics: 5239, pp 26-32.

152. Amini, J.; Saradjian, M.; Blais, J.; Lucas, C.; Azizi, A. Automatic Road-Side Extraction from Large Scale Imagemaps. International Journal of Applied Earth Observation and Geoinformation 2002, 4, 95-107.

153. Drăgut,, L.; Blaschke, T. Automated Classification of Landform Elements Using Object-Based I mage Analysis. Geomorphology 2006, 81, 330-344.

154. Saeedi, P.; Zwick, H. In Automatic Building Detection in Aerial and Satellite Images, International Conference on Control, Automation, Robotics and Vision, Phuket, Thailand, 2008; pp 623-629.

155. Song, Z.; Pan, C.; Yang, Q. In A Region-Based Approach to Building Detection in Densely Build-up High Resolution Satellite Image, IEEE International Conference on Image Processing, Atlanta, USA, 2006; pp 3225-3228.

156. Song, M.; Civco, D. Road Extraction Using Svm and Image Segmentation. Photogrammetric Engineering \& Remote Sensing 2004, 70, 1365-1371.

157. Momm, H.; Gunter, B.; Easson, G. In Improved Feature Extraction from High-Resolution Remotely Sensed Imagery Using Object Geometry, SPIE - International Society for Optical Engineering, 2010; 7695, pp 1-11.

158. Chaudhuri, D.; Kushwaha, N.; Samal, A. Semi-Automated Road Detection from High Resolution Satellite Images by Directional Morphological Enhancement and Segmentation Techniques. IEEE Journal of Selected Topics in Applied Earth Observations and Remote Sensing 2012, 5, 1538-1544.

159. Hofmann, P. Detecting Buildings and Roads from Ikonos Data Using Additional Elevation Information. GIS 2001, 6, 28-33.

160. Hofmann, P. In Detecting Urban Features from Ikonos Data Using an Object-Oriented Approach, Proceedings of the first annual conference of the remote sensing \& photogrammetry society, Nottingham, UK, 12-14 September, 2001; pp 79-91.

161. Yager, N.; Sowmya, A. In Support Vector Machines for Road Extraction from Remotely Sensed Images, Computer Analysis of Images and Patterns, York, UK, 27-29 August 2003; pp 285292. 
162. Wang, Y.; Tian, Y.; Tai, X.; Shu, L. Extraction of Main Urban Roads from High Resolution Satellite Images by Machine Learning. In Computer Vision-Accv, Springer: 2006; pp 236245.

163. Zhao, B.; Zhong, Y.; Zhang, L. A Spectral-Structural Bag-ofFeatures Scene Classifier for Very High Spatial Resolution Remote Sensing Imagery. ISPRS Journal of Photogrammetry and Remote Sensing 2016, 116, 73-85.

164. Gerke, M.; Xiao, J. In Supervised and Unsupervised Mrf Based 3D Scene Classification in Multiple View Airborne Oblique Images, ISPRS Annals of the Photogrammetry, Remote Sensing and Spatial Information Sciences, Antalya, Turkey, 12-13 November, 2013; II-3/W3, pp 25-30.

165. Gerke, M.; Xiao, J. Fusion of Airborne Laserscanning Point Clouds and Images for Supervised and Unsupervised Scene Classification. ISPRS J ournal of Photogrammetry and Remote Sensing 2014, 87, 78-92.

166. Guindon, B. In Application of Spatial Reasoning Methods to the Extraction of Roads from High Resolution Satellite Imagery, IEEE International Geoscience and Remote Sensing Symposium (IGARSS), Seattle, WA, USA, 6-10 July, 1998; 2, pp 1076-1078.

167. Rydberg, A.; Borgefors, G. Integrated Method for Boundary Delineation of Agricultural Fields in Multispectral Satellite Images. IEEE Transactions on Geoscience and Remote Sensing 2001, 39, 2514-2520.

168. Mokhtarzade, M.; Ebadi, H.; Valadan Zoej, M. Optimization of Road Detection from High-Resolution Satellite Images Using Texture Parameters in Neural Network Classifiers. Canadian J ournal of Remote Sensing 2007, 33, 481-491.

169. Mokhtarzade, M.; Zoej, M.V. Road Detection from HighResolution Satellite Images Using Artificial Neural Networks. International Journal of Applied Earth Observation and Geoinformation 2007, 9, 32-40.

170. Zheng, Y.-J. Feature Extraction and I mage Segmentation Using Self-Organizing Networks. Machine Vision and Applications 1995, 8, 262-274.

171. Canny, J. A Computational Approach to Edge Detection. IEEE Transactions on Pattern Analysis and Machine Intelligence 1986, PAMI-8, 679-698.

172. Juneja, M.; Sandhu, P.S. Performance Evaluation of Edge Detection Techniques for Images in Spatial Domain. International Journal of Computer Theory and Engineering 2009, 1, 614-621.

173. Shrivakshan, G.T.; Chandrasekar, C. A Comparison of Various Edge Detection Techniques Used in Image Processing. 
International J ournal of Computer Science I ssues 2012, 9, 269276.

174. Hough, P.V. Method and Means for Recognizing Complex Patterns. US Patent 30696541962.

175. Wu, J.; Jie, S.; Yao, W.; Stilla, U. In Building Boundary Improvement for True Orthophoto Generation by Fusing Airborne Lidar Data, Joint Urban Remote Sensing Event (JURSE), Munich, Germany, 11-13 April, 2011; pp 125-128.

176. von Gioi, R.G.; Jakubowicz, J.; Morel, J.-M.; Randall, G. Lsd: A Line Segment Detector. I mage Processing On Line 2012, 2, 3555.

177. Babawuro, U.; Beiji, Z. Satellite Imagery Quality Evaluation Using I mage Quality Metrics for Quantitative Cadastral Analysis. International Journal of Computer Applications in Engineering Sciences 2011, 1, 391-395.

178. Turker, M.; Kok, E.H. Field-Based Sub-Boundary Extraction from Remote Sensing Imagery Using Perceptual Grouping. ISPRS J ournal of Photogrammetry and Remote Sensing 2013, 79, 106-121.

179. Hu, J.; You, S.; Neumann, U. In Integrating Lidar, Aerial I mage and Ground Images for Complete Urban Building Modeling, International Symposium on 3D Data Processing, Visualization, and Transmission, Chapel Hill, USA, 14-16 J une, 2006; pp 184191.

180. Hu, J.; You, S.; Neumann, U.; Park, K.K. In Building Modeling from Lidar and Aerial I magery, Proceedings of ASPRS, 2004; 4, pp 23-28.

181. Liu, Z.; Cui, S.; Yan, Q. In Building Extraction from High Resolution Satellite Imagery Based on Multi-Scale Image Segmentation and Model Matching, International Workshop on Earth Observation and Remote Sensing Applications, Beijing, China, 30 June-2 July, 2008; pp 1-7.

182. Bartl, R.; Petrou, M.; Christmas, W.J .; Palmer, P. In Automatic Registration of Cadastral Maps and Landsat Tm Images, Satellite Remote Sensing III, Taormina, Italy, 17 December, 1996; SPIE: 2955, pp 9-20.

183. Wang, Z.; Liu, W. In Building Extraction from High Resolution Imagery Based on Multi-Scale Object Oriented Classification and Probabilistic Hough Transform, International Geoscience and Remote Sensing Symposium (IGARSS), Seoul, South Korea, 31 July-4 August, 2005; pp 25-29.

184. Park, S.-R.; Kim, T. In Semi-Automatic Road Extraction Algorithm from I konos Images Using Template Matching, Asian Conference on Remote Sensing, Singapore, 5-9 November, 2001; 5, p 9. 
185. Goshtasby, A.; Shyu, H.-L. Edge Detection by Curve Fitting. Image and Vision Computing 1995, 13, 169-177.

186. Venkateswar, V.; Chellappa, R. Extraction of Straight Lines in Aerial Images. IEEE Transactions on Pattern Analysis \& Machine Intelligence 1992, 1111-1114.

187. Lu, H.; Aggarwal, J.K. Applying Perceptual Organization to the Detection of Man-Made Objects in Non-Urban Scenes. Pattern Recognition 1992, 25, 835-853.

188. Torre, M.; Radeva, P. In Agricultural Field Extraction from Aerial Images Using a Region Competition Algorithm, ISPRS International Archives of the Photogrammetry, Remote Sensing and Spatial Information Sciences, Amsterdam, Netherlands, 2000; XXXIII-B3, pp 889-896.

189. Vosselman, G.; de Knecht, J. Road Tracing by Profile Matching and Kalman Filtering. In Automatic Extraction of Man-Made Objects from Aerial and Space Images, Gruen, A.; Kuebler, O.; Agouris, P., Eds. Springer: Boston, 1995; pp 265-274.

190. Sarkar, S.; Boyer, K.L. Perceptual Organization in Computer Vision: A Review and a Proposal for a Classificatory Structure. IEEE Transactions on Systems, Man and Cybernetics 1993, 23, 382-399.

191. Kass, M.; Witkin, A.; Terzopoulos, D. Snakes: Active Contour Models. International J ournal of Computer Vision 1988, 1, 321331.

192. Hasegawa, H. In A Semi Automatic Road Extraction Method for Alos Satellite Imagery, ISPRS - International Archives of the Photogrammetry, Remote Sensing and Spatial Information Sciences, Istanbul, Turkey, 2004; XXXV-3, p 303.

193. Montesinos, P.; Alquier, L. In Perceptual Organization of Thin Networks with Active Contour Functions Applied to Medical and Aerial I mages, International Conference on Pattern Recognition, Vienna, Austria, 25-30 August, 1996; 1, pp 647-651.

194. Yang, J.; Wang, R. Classified Road Detection from Satellite Images Based on Perceptual Organization. International J ournal of Remote Sensing 2007, 28, 4653-4669.

195. Mohan, R.; Nevatia, R. Using Perceptual Organization to Extract 3D Structures. IEEE Transactions on Pattern Analysis and Machine Intelligence 1989, 11, 1121-1139.

196. Jaynes, C.O.; Stolle, F.; Collins, R.T. In Task Driven Perceptual Organization for Extraction of Rooftop Polygons, Second IEEE Workshop on Applications of Computer Vision, Sarasota, USA, 1994; pp 152-159.

197. Lin, C.; Huertas, A.; Nevatia, R. In Detection of Buildings Using Perceptual Grouping and Shadows, IEEE Conference on 
Computer Vision and Pattern Recognition (CVPR), Seattle, WA, USA, 1994; pp 62-69.

198. Noronha, S.; Nevatia, R. In Detection and Description of Buildings from Multiple Aerial Images, IEEE Conference on Computer Vision and Pattern Recognition (CVPR), Fort Collins, USA, 1997; pp 588-594.

199. Mayer, H.; Laptev, I.; Baumgartner, A. Multi-Scale and Snakes for Automatic Road Extraction. In Computer Vision-Eccv, Springer: 1998; pp 720-733.

200. Gruen, A.; Li, H. Semi-Automatic Linear Feature Extraction by Dynamic Programming and LSB-Snakes. Photogrammetric engineering and remote sensing 1997, 63, 985-994.

201. Laptev, I.; Mayer, H.; Lindeberg, T.; Eckstein, W.; Steger, C.; Baumgartner, A. Automatic Extraction of Roads from Aerial Images Based on Scale Space and Snakes. Machine Vision and Applications 2000, 12, 23-31.

202. Agouris, P.; Gyftakis, S.; Stefanidis, A. In Dynamic Node Distribution in Adaptive Snakes for Road Extraction, Vision Interface, Ottawa, Canada, 7-9 June, 2001; pp 134-140.

203. Saalfeld, A. Topologically Consistent Line Simplification with the Douglas-Peucker Algorithm. Cartography and Geographic Information Science 1999, 26, 7-18.

204. Dong, P. Implementation of Mathematical Morphological Operations for Spatial Data Processing. Computers \& Geosciences 1997, 23, 103-107.

205. Guo, X.; Dean, D.; Denman, S.; Fookes, C.; Sridharan, S. In Evaluating Automatic Road Detection across a Large Aerial Imagery Collection, International Conference on Digital Image Computing Techniques and Applications, Noosa, Australia, 6-8 December, 2011; pp 140-145.

206. Heipke, C.; Englisch, A.; Speer, T.; Stier, S.; Kutka, R. Semiautomatic Extraction of Roads from Aerial Images. In Spatial I nformation from Digital Photogrammetry and Computer Vision: ISPRS Commission Iii Symposium, 1994; Vol. 2357, pp 353-360.

207. Amini, J.; Lucas, C.; Saradjian, M.; Azizi, A.; Sadeghian, S. Fuzzy Logic System for Road Identification Using Ikonos Images. The Photogrammetric Record 2002, 17, 493-503.

208. Ziems, M.; Gerke, M.; Heipke, C. In Automatic Road Extraction from Remote Sensing I magery Incorporating Prior I nformation and Colour Segmentation, ISPRS - International Archives of the Photogrammetry, Remote Sensing and Spatial Information Sciences, Munich, Germany, 19-21 September, 2007; XXXVI3/W49a, pp 141-147. 
209. Mohammadzadeh, A.; Tavakoli, A.; Zoej, M.V. In Automatic Linear Feature Extraction of I ranian Roads from High Resolution Multi-Spectral Satellite I magery, ISPRS Congress: Geo-Imagery Bridging Continents, Istanbul, Turkey, 12-23 July, 2004; pp 764-768.

210. Corcoran, P.; Winstanley, A.; Mooney, P. Segmentation Performance Evaluation for Object-Based Remotely Sensed Image Analysis. International J ournal of Remote Sensing 2010, 31, 617-645.

211. Baumgartner, A.; Steger, C.; Mayer, H.; Eckstein, W.; Ebner, H. Automatic Road Extraction Based on Multi-Scale, Grouping, and Context. Photogrammetric Engineering \& Remote Sensing 1999, 65, 777-786.

212. Wiedemann, C. In External Evaluation of Road Networks, ISPRS - International Archives of the Photogrammetry, Remote Sensing and Spatial Information Sciences, Munich, Germany, 2003; XXXIV-3/W8, pp 93-98.

213. Overby, J.; Bodum, L.; Kjems, E.; Iisoe, P. Automatic 3D Building Reconstruction from Airborne Laser Scanning and Cadastral Data Using Hough Transform. ISPRS - International Archives of the Photogrammetry, Remote Sensing and Spatial Information Sciences 2004, 35, 296-301.

214. Radoux, J.; Bogaert, P.; Fasbender, D.; Defourny, P. Thematic Accuracy Assessment of Geographic Object-Based Image Classification. International J ournal of Geographical I nformation Science 2011, 25, 895-911.

215. Stehman, S.V. Selecting and Interpreting Measures of Thematic Classification Accuracy. Remote Sensing of Environment 1997, 62, 77-89.

216. Zhan, Q.; Molenaar, M.; Tempfli, K.; Shi, W. Quality Assessment for Geo-Spatial Objects Derived from Remotely Sensed Data. International Journal of Remote Sensing 2005, 26, 2953-2974.

217. Foody, G.M. Status of Land Cover Classification Accuracy Assessment. Remote Sensing of Environment 2002, 80, 185201.

218. Congalton, R.G.; Green, K. Assessing the Accuracy of Remotely Sensed Data: Principles and Practices. CRC press: 2008; p 200.

219. Lizarazo, I. Accuracy Assessment of Object-Based Image Classification: Another Step. International Journal of Remote Sensing 2014, 35, 6135-6156.

220. Rottensteiner, F.; Sohn, G.; Gerke, M.; Wegner, J.D.; Breitkopf, U.; Jung, J. Results of the ISPRS Benchmark on Urban Object Detection and 3D Building Reconstruction. ISPRS Journal of Photogrammetry and Remote Sensing 2014, 93, 256-271. 
221. Winter, S. Uncertain Topological Relations between Imprecise Regions. International Journal of Geographical Information Science 2000, 14, 411-430.

222. Clementini, E.; Di Felice, P. An Algebraic Model for Spatial Objects with Indeterminate Boundaries. In Geographic Objects with Indeterminate Boundaries, Burrough, P.A.; Frank, A., Eds. Taylor \& Francis: London, 1996; pp 155-169.

223. Worboys, M. Imprecision in Finite Resolution Spatial Data. Geol nformatica 1998, 2, 257-279.

224. Rutzinger, M.; Rottensteiner, F.; Pfeifer, N. A Comparison of Evaluation Techniques for Building Extraction from Airborne Laser Scanning. IEEE J ournal of Selected Topics in Applied Earth Observations and Remote Sensing 2009, 2, 11-20.

225. Heipke, C.; Mayer, H.; Wiedemann, C.; Jamet, O. In Evaluation of Automatic Road Extraction, ISPRS - International Archives of the Photogrammetry, Remote Sensing and Spatial Information Sciences, Stuttgart, Germany, 1997; XXXII-3-4W2, pp 151160.

226. Wiedemann, C.; Ebner, H. In Automatic Completion and Evaluation of Road Networks, ISPRS - International Archives of the Photogrammetry, Remote Sensing and Spatial Information Sciences, Amsterdam, Netherlands, 2000; XXXIII-3, pp 979986.

227. Harvey, W.A. Performance Evaluation for Road Extraction. Bulletin de la Société Française de Photogrammétrie et Télédétection 1999, 153, 79-87.

228. Shi, W.; Cheung, C.K.; Zhu, C. Modelling Error Propagation in Vector-Based Buffer Analysis. International Journal of Geographical Information Science 2003, 17, 251-271.

229. Suetens, P.; Fua, P.; Hanson, A.J. Computational Strategies for Object Recognition. ACM Computing Surveys 1992, 24, 5-62.

230. Mena, J.B. State of the Art on Automatic Road Extraction for GIS Update: A Novel Classification. Pattern Recognition Letters 2003, 24, 3037-3058.

231. Tien, D.; Jia, W. In Automatic Road Extraction from Aerial Images: A Contemporary Survey, International Conference in IT and Applications (ICITA), Harbin, China, 2007; pp 294-299.

232. Mayer, H.; Hinz, S.; Bacher, U.; Baltsavias, E. A Test of Automatic Road Extraction Approaches. ISPRS - International Archives of the Photogrammetry, Remote Sensing and Spatial Information Sciences 2006, XXXVI-3, 209-214.

233. Xie, S.; Tu, Z. In Holistically-Nested Edge Detection, IEEE International Conference on Computer Vision, Santiago, Chile, 13-16 December, 2015; pp 1395-1403. 
234. Arbelaez, P.; Maire, M.; Fowlkes, C.; Malik, J. Contour Detection and Hierarchical Image Segmentation. Pattern Analysis and Machine Intelligence, IEEE Transactions on 2011, 33, 898-916.

235. Vetrivel, A.; Gerke, M.; Kerle, N.; Vosselman, G. Identification of Structurally Damaged Areas in Airborne Oblique Images Using a Visual-Bag-of-Words Approach. Remote Sensing 2016, $8,231$.

236. Gülch, E.; Müller, H.; Hahn, M. In Semi-Automatic Object Extraction-Lessons Learned, ISPRS - International Archives of the Photogrammetry, Remote Sensing and Spatial Information Sciences, 2004; XXXIV-3, pp 488-493.

237. Hecht, A.D.; Fiksel, J.; Fulton, S.C.; Yosie, T.F.; Hawkins, N.C.; Leuenberger, H.; Golden, J.S.; Lovejoy, T.E. Creating the Future We Want. Sustainability: Science, Practice, and Policy 2012, 8, 62-75.

238. Baltsavias, E.P. Object Extraction and Revision by Image Analysis Using Existing Geodata and Knowledge: Current Status and Steps Towards Operational Systems. ISPRS Journal of Photogrammetry and Remote Sensing 2004, 58, 129-151.

239. Schwering, A.; Wang, J.; Chipofya, M.; Jan, S.; Li, R.; Broelemann, K. Sketchmapia: Qualitative Representations for the Alignment of Sketch and Metric Maps. Spatial Cognition and Computation 2014, 14, 220-254.

240. Crommelinck, S.; Bennett, R.; Gerke, M.; Yang, M.Y.; Vosselman, G. Contour Detection for UAV-Based Cadastral Mapping. Remote Sensing 2017, 9, 1-13.

241. Manyoky, M.; Theiler, P.; Steudler, D.; Eisenbeiss, H. In Unmanned Aerial Vehicle in Cadastral Applications, International Archives of the Photogrammetry, Remote Sensing and Spatial Information Sciences, Zurich, Switzerland, 14-16 September, 2011; XXXVIII-1/C22, pp 1-6.

242. Mumbone, M.; Bennett, R.; Gerke, M.; Volkmann, W. In Innovations in Boundary Mapping: Namibia, Customary Lands and UAVs, World Bank Conference on Land and Poverty, Washington, DC, USA, 23-27 March, 2015; pp 1-22.

243. Volkmann, W.; Barnes, G. In Virtual Surveying: Mapping and Modeling Cadastral Boundaries Using Unmanned Aerial Systems (UAS), FIG Congress, Kuala Lumpur, Malaysia, 16-21 June, 2014; pp 1-13.

244. Malik, J.; Belongie, S.; Leung, T.; Shi, J. Contour and Texture Analysis for Image Segmentation. International Journal of Computer Vision 2001, 43, 7-27.

245. Maire, M.; Arbeláez, P.; Fowlkes, C.; Malik, J. In Using Contours to Detect and Localize Junctions in Natural Images, IEEE 
Conference on Computer Vision and Pattern Recognition (CVPR'08), Anchorage, AK, USA, 23-28 June, 2008; pp 1-8.

246. Arbelaez, P.; Maire, M.; Fowlkes, C.; Malik, J. In From Contours to Regions: An Empirical Evaluation, IEEE Conference on Computer Vision and Pattern Recognition (CVPR), Miami Beach, FL, USA, 20-25 June, 2009; pp 2294-2301.

247. Arbeláez, P.; Fowlkes, C.; Martin, D. Berkeley Segmentation Dataset and Benchmark. Available online: https://www2.eecs. berkeley. edu/Research/Projects/CS/vision/ bsds/ (accessed on 10 July 2019).

248. Arbeláez, P.; Maire, M.; Fowlkes, C.; Malik, J. Contour Detection and Image Segmentation Resources. Available online: https://www2.eecs.berkeley.edu/Research/Projects/CS/vision/ grouping/resources. html (accessed on 10 July 2019).

249. Arbeláez, P. In Boundary Extraction in Natural Images Using Ultrametric Contour Maps, Conference on Computer Vision and Pattern Recognition Workshop (CVPRW), New York, NY, USA, 17-22 June, 2006; pp 1-8.

250. Jevnisek, R.J.; Avidan, S. Semi Global Boundary Detection. Computer Vision and Image Understanding 2016, 152, 21-28.

251. Zhang, X.; Xiao, P.; Song, X.; She, J. Boundary-Constrained Multi-Scale Segmentation Method for Remote Sensing Images. ISPRS J ournal of Photogrammetry and Remote Sensing 2013, 78, 15-25.

252. Szeliski, R. Computer Vision: Algorithms and Applications. Springer London: London, UK, 2010; p 812.

253. Dornaika, F.; Moujahid, A.; El Merabet, Y.; Ruichek, Y. Building Detection from Orthophotos Using a Machine Learning Approach: An Empirical Study on Image Segmentation and Descriptors. Expert Systems with Applications 2016, 58, 130142.

254. Hou, B.; Kou, H.; Jiao, L. Classification of Polarimetric Sar Images Using Multilayer Autoencoders and Superpixels. IEEE Journal of Selected Topics in Applied Earth Observations and Remote Sensing 2016, 9, 3072 - 3081.

255. Wiedemann, C.; Heipke, C.; Mayer, H.; Jamet, O. Empirical Evaluation of Automatically Extracted Road Axes. In Empirical Evaluation Techniques in Computer Vision, Bowyer, K.W.; Phillips, P.J., Eds. IEEE Computer Society Press: Los Alamitos, CA, USA, 1998; pp 172- 187.

256. Mayer, H. Object Extraction in Photogrammetric Computer Vision. ISPRS J ournal of Photogrammetry and Remote Sensing 2008, 63, 213-222.

257. Kumar, M.; Singh, R.; Raju, P.; Krishnamurthy, Y. In Road Network Extraction from High Resolution Multispectral Satellite 
Imagery Based on Object Oriented Techniques, ISPRS Technical Commission VIII Symposium, Hyderabad, India, 09-12 December, 2014; II-8, pp 107-110.

258. Goodchild, M.F.; Hunter, G.J. A Simple Positional Accuracy Measure for Linear Features. International Journal of Geographical Information Science 1997, 11, 299-306.

259. Zevenbergen, J.; De Vries, W.; Bennett, R. Advances in Responsible Land Administration. CRC Press: Padstow, UK, 2015; p 279.

260. Basaeed, E.; Bhaskar, H.; Al-Mualla, M. In Cnn-Based MultiBand Fused Boundary Detection for Remotely Sensed I mages, International Conference on Imaging for Crime Prevention and Detection, London, UK, 15-17 July, 2015; pp 1-6.

261. Crommelinck, S.; Bennett, R.; Gerke, M.; Koeva, M.; Yang, M.Y.; Vosselman, G. In SLIC Superpixels for Object Delineation from UAV Data, International Conference on Unmanned Aerial Vehicles in Geomatics, Bonn, Germany, 04-07 September, 2017; IV-2/W3, pp 9-16.

262. Ren, X.; Malik, J. In Learning a Classification Model for Segmentation, International Conference on Computer Vision (ICCV), Washington, DC, USA, 13-16 October, 2003; pp 10-17.

263. Csillik, O. In Superpixels: The End of Pixels in Obia. A Comparison of Stat-of-the-Art Superpixel Methods for Remote Sensing Data, GEOBIA, Enschede, Netherlands, 14-16 September, 2016; pp 1-5.

264. Neubert, P.; Protzel, P. In Superpixel Benchmark and Comparison, Proc. Forum Bildverarbeitung, Regensburg, Germany, 29-30 November, 2012; pp 1-12.

265. Stutz, D. In Superpixel Segmentation: An Evaluation, German Conference on Pattern Recognition (GCPR), Aachen, Germany, 7-10 October, 2015; pp 555-562.

266. Schick, A.; Fischer, M.; Stiefelhagen, R. In Measuring and Evaluating the Compactness of Superpixels, International Conference on Pattern Recognition (ICPR), Tsukuba Science City, Japan, 11-15 November, 2012; pp 930-934.

267. Stutz, D.; Hermans, A.; Leibe, B. Superpixels: An Evaluation of the State-of-the-Art. Computer Vision and Image Understanding 2017, 1-32.

268. Achanta, R.; Shaji, A.; Smith, K.; Lucchi, A.; Fua, P.; Süsstrunk, S. In SLIC Superpixels, EPFL Technical Report no. 149300, 2010; pp 1-15.

269. Shi, J.; Malik, J. Normalized Cuts and Image Segmentation. IEEE Transactions on Pattern Analysis and Machine Intelligence 2000, 22, 888-905. 
270. Moore, A.P.; Prince, S.J.; Warrell, J.; Mohammed, U.; J ones, G. In Superpixel Lattices, IEEE Conference on Computer Vision and Pattern Recognition (CVPR), Anchorage, AK, USA, 23-28 June, 2008; pp 1-8.

271. Comaniciu, D.; Meer, P. Mean Shift: A Robust Approach toward Feature Space Analysis. IEEE Transactions on Pattern Analysis and Machine Intelligence 2002, 24, 603-619.

272. Levinshtein, A.; Stere, A.; Kutulakos, K.N.; Fleet, D.J.; Dickinson, S.J .; Siddiqi, K. Turbopixels: Fast Superpixels Using Geometric Flows. IEEE Transactions on Pattern Analysis and Machine Intelligence 2009, 31, 2290-2297.

273. Vincent, L.; Soille, P. Watersheds in Digital Spaces: An Efficient Algorithm Based on Immersion Simulations. IEEE Transactions on Pattern Analysis and Machine Intelligence 1991, 13, 583598.

274. Gary, B. Opencv Superpixels. Available online: http://docs.opencv.org/trunk/df/d6c/group ximgproc super pixel.html (accessed on 10 July 2019).

275. Vedaldi, A. VIfeat SLIC Superpixels. Available online: http://www.vlfeat.org/api/slic.html (accessed on 10 July 2019).

276. Balint, C. Gdal Superpixels. Available online: https://github.com/cbalint13/gdal-segment (accessed on 10 July 2019).

277. Scikit-Learn Developers. Scikit SLIC Superpixels. Available online: http://scikitimage.org/docs/dev/api/skimage. segmentation. html\#skimage segmentation. slic (accessed on 10 July 2019).

278. MathWorks. Matlab Superpixels. Available online: https://nl. mathworks.com/help/images/ref/superpixels.html (accessed on 10 July 2019).

279. Kanavath, R.; Metz, M. Grass SLIC Superpixels. Available online:

https://grass. osgeo.org/grass72/manuals/addons/i.superpixel s.slic.html (accessed on 10 July 2019).

280. Malisiewicz, T.; Efros, A.A. In Improving Spatial Support for Objects Via Multiple Segmentations, British Machine Vision Conference (BMVC), Coventry, UK, 10-13 September 2007, 2007; pp 1-11.

281. Pantofaru, C.; Schmid, C.; Hebert, M. In Object Recognition by Integrating Multiple Image Segmentations, European Conference on Computer Vision (ECCV), Marsaille, France, 1218 October, 2008; pp 481-494.

282. Zhang, G.; Jia, X.; Hu, J. Superpixel-Based Graphical Model for Remote Sensing Image Mapping. IEEE Transactions on Geoscience and Remote Sensing 2015, 53, 5861-5871. 
283. Vargas, J.; Falcao, A.; dos Santos, J.; Esquerdo, J.; Coutinho, A.; Antunes, J. In Contextual Superpixel Description for Remote Sensing Image Classification, IEEE International Geoscience and Remote Sensing Symposium (IGARSS), Milan, Italy, 26-31 July, 2015; pp 1132-1135.

284. Thompson, D.R.; Mandrake, L.; Gilmore, M.S.; Castano, R. Superpixel Endmember Detection. IEEE Transactions on Geoscience and Remote Sensing 2010, 48, 4023-4033.

285. Ortiz Toro, C.A.; Gonzalo Martín, C.; García Pedrero, Á.; Menasalvas Ruiz, E. Superpixel-Based Roughness Measure for Multispectral Satellite Image Segmentation. Remote Sensing 2015, 7, 14620-14645.

286. Acuña, R.G.G.; Tao, J.; Breen, D.; Breen, B.; Pointing, S.; Gillman, L.; Klette, R. Robust Segmentation of Aerial Image Data Recorded for Landscape Ecology Studies. In Image and Video Technology, Huang, F.; Sugimoto, A., Eds. Springer: Cham, Switzerland, 2016; pp 61-72.

287. Sahli, S.; Lavigne, D.A.; Sheng, Y. Saliency Region Selection in Large Aerial Imagery Using Multiscale SLIC Segmentation. Proceedings of SPIE 2012, 8360, 1-8.

288. Chen, Z.; Wang, C.; Wen, C.; Teng, X.; Chen, Y.; Guan, H.; Luo, H.; Cao, L.; Li, J. Vehicle Detection in High-Resolution Aerial Images Via Sparse Representation and Superpixels. IEEE Transactions on Geoscience and Remote Sensing 2016, 54, 103-116.

289. Chen, Y.; Qin, K.; Liu, Y.; Gan, S.; Zhan, Y. Feature Modelling of High Resolution Remote Sensing I mages Considering Spatial Autocorrelation. ISPRS - International Archives of the Photogrammetry, Remote Sensing and Spatial Information Sciences 2012, Volume XXXIX-B3, 467-472.

290. Csilik, O.; Lang, S. In Improving the Speed of Multiresolution Segmentation Using SLIC Superpixels, GEOBIA, Enschede, Netherlands, 14-16 September, 2016; pp 1-4.

291. Russell, B.C.; Freeman, W.T.; Efros, A.A.; Sivic, J.; Zisserman, A. In Using Multiple Segmentations to Discover Objects and Their Extent in Image Collections, IEEE Conference on Computer Vision and Pattern Recognition (CVPR), New York, NY, USA, 17-22 June, 2006; pp 1605-1614.

292. Borenstein, E.; Ullman, S. Combined Top-Down/Bottom-up Segmentation. IEEE Transactions on Pattern Analysis and Machine Intelligence 2008, 30, 2109-2125.

293. Yang, M.Y.; Rosenhahn, B. Superpixel Cut for Figure-Ground Image Segmentation. ISPRS Annals of the Photogrammetry, Remote Sensing and Spatial Information Sciences 2016, III-3, 387-394. 
294. Levinshtein, A.; Sminchisescu, C.; Dickinson, S. Optimal Image and Video Closure by Superpixel Grouping. International J ournal of Computer Vision 2012, 100, 99-119.

295. Levinshtein, A.; Sminchisescu, C.; Dickinson, S. In Optimal Contour Closure by Superpixel Grouping, European Conference on Computer Vision (ECCV), Crete, Greece, 5-11 September, 2010; pp 480-493.

296. QGIS Development Team. QGIS Geographic Information System, Open Source Geospatial Foundation. Available online: https://www.qgis.org (accessed on 10 October 2019).

297. GRASS Development Team. Geographic Resources Analysis Support System (Grass) Software, Version 7.0. Available online: https://grass.osgeo.org/ (accessed on 10 July 2019).

298. GDAL Development Team. Gdal - Geospatial Data Abstraction Library, Version 2.1.2. Open Source Geospatial Foundation. Available online: www.gdal.org (accessed on 10 July 2019).

299. Wiedemann, C. In External Evaluation of Road Networks, Photogrammetric image analysis, Munich, Germany, 17-19 December, 2003; XXXIV-3/W8, pp 93-98.

300. Crommelinck, S.; Höfle, B.; Koeva, M.; Yang, M.Y.; Vosselman, G. In Interactive Boundary Delineation from UAV Data, ISPRS Annals of the Photogrammetry, Remote Sensing and Spatial Information Sciences, Riva del Garda, Italy, 04-07 June, 2018; IV-2, pp 81-88.

301. Koeva, M.; Muneza, M.; Gevaert, C.; Gerke, M.; Nex, F. Using UAVs for Map Creation and Updating. A Case Study in Rwanda. Survey Review 2016, 1-14.

302. Gevaert, C.M. Unmanned Aerial Vehicle Mapping for Settlement Upgrading. PhD, University of Twente, 2018.

303. International Association of Assessing Officers (IAAO). Standard on Digital Cadastral Maps and Parcel Identifiers. Kansas City, MO, USA, 2015; p 24.

304. Luo, X.; Bennett, R.; Koeva, M.; Lemmen, C.; Quadros, N. Quantifying the Overlap between Cadastral and Visual Boundaries: A Case Study from Vanuatu. Urban Science 2017, $1,32$.

305. Crommelinck, S. Delineation-Tool Github. Available online: https://github.com/its4land/delineation-tool (accessed on 10 October 2019).

306. Crommelinck, S. QGIS Plugin Repository: Boundarydelineation. Available online: http://plugins.qgis.org/plugins/BoundaryDelineation/ (accessed on 10 October 2019).

307. Sowmya, A.; Trinder, J. Modelling and Representation Issues in Automated Feature Extraction from Aerial and Satellite Images. 
ISPRS J ournal of Photogrammetry and Remote Sensing 2000, 55, 34-47.

308. Iqbal, Q.; Aggarwal, J.K. Retrieval by Classification of Images Containing Large Manmade Objects Using Perceptual Grouping. Pattern Recognition 2002, 35, 1463-1479.

309. Stahl, J.S.; Wang, S. Edge Grouping Combining Boundary and Region Information. IEEE Transactions on Image Processing 2007, 16, 2590-2606.

310. Estrada, F.J .; Jepson, A.D. In Perceptual Grouping for Contour Extraction, International Conference on Pattern Recognition (ICPR), Cambridge, UK, 26-26 August, 2004; 2, pp 32-35.

311. Lowe, D. Perceptual Organization and Visual Recognition. Springer: 1985; Vol. 5, p 162.

312. Warnke, S.; Bulatov, D. In Variable Selection for Road Segmentation in Aerial Images, International Archives of the Photogrammetry, Remote Sensing and Spatial Information Sciences, Hannover, Germany, 6-9 June, 2017; XLII-1/W1, pp 297-304.

313. García-Pedrero, A.; Gonzalo-Martín, C.; Lillo-Saavedra, M. A Machine Learning Approach for Agricultural Parcel Delineation through Agglomerative Segmentation. International J ournal of Remote Sensing 2017, 38, 1809-1819.

314. Santana, T.M.H.C.; Machado, A.M.C.; de A. Araújo, A.; dos Santos, J.A. In Star: A Contextual Description of Superpixels for Remote Sensing Image Classification, Progress in Pattern Recognition, Image Analysis, Computer Vision, and Applications, Lima, Peru, 8-11 November, 2017; pp 300-308.

315. Wang, X.-Y.; Wu, C.-W.; Xiang, K.; Chen, W. Efficient Local and Global Contour Detection Based on Superpixels. Journal of Visual Communication and Image Representation 2017, 48, 77-87.

316. Jiang, H.; Wu, Y.; Yuan, Z. In Probabilistic Salient Object Contour Detection Based on Superpixels, Conference on Image Processing (ICIP), Melbourne, Australia, 15-18 September, 2013; pp 3069-3072.

317. Stock, K.M. Accuracy Requirements for Rural Land Parcel Boundaries. Australian Surveyor 1998, 43, 165-171.

318. Albrecht, F. In Uncertainty in I mage Interpretation as Reference for Accuracy Assessment in Object-Based Image Analysis, International Symposium on Spatial Accuracy Assessment in Natural Resources and Environmental Sciences, Leicester, UK, 20-23 July, 2010; pp 13-16.

319. Genuer, R.; Poggi, J.-M.; Tuleau-Malot, C. Variable Selection Using Random Forests. Pattern Recognition Letters 2010, 31, 2225-2236. 
320. Crommelinck, S.; Koeva, M.; Yang, M.Y.; Vosselman, G. Robust Object Extraction from Remote Sensing Data. arXiv: 1904.125862019.

321. Breiman, L. Random Forests. Machine Learning 2001, 45, 532.

322. Pedregosa, F.; Varoquaux, G.; Gramfort, A.; Michel, V.; Thirion, B.; Grisel, O.; Blondel, M.; Prettenhofer, P.; Weiss, R.; Dubourg, V. Scikit-Learn: Machine Learning in Python. Journal of Machine Learning Research 2011, 12, 2825-2830.

323. Crommelinck, S.; Yang, M.Y.; Koeva, M.; Gerke, M.; Bennett, R.; Vosselman, G. Towards Automated Cadastral Boundary Delineation from UAV Data. arXiv: 1709.018132017.

324. Puniach, E.; Bieda, A.; Ćwiakała, P.; Kwartnik-Pruc, A.; Parzych, P. Use of Unmanned Aerial Vehicles (UAVs) for Updating Farmland Cadastral Data in Areas Subject to Landslides. ISPRS International Journal of Geo-Information 2018, 7, 1-19.

325. Heipke, C.; Mayer, H.; Wiedemann, C.; Jamet, O. In Evaluation of Automatic Road Extraction, 3D Reconstrucion and Modeling of Topographic Objects, Stuttgart, Germany, 17-19 September, 1997; XXXII-3-4W2, pp 151-160.

326. Crommelinck, S. QGIS Python Plugins Repository: Linecomparison. Available online: https://plugins.qgis.org/plugins/linecomparison/ (accessed on 10 July 2019).

327. Siriba, D.N.; Voß, W.; Mulaku, G.C. The Kenyan Cadastre and Modern Land Administration. Zeitschrift fur Vermessungswesen 2011, 136, 177-186.

328. Mwenda, J.N. In Spatial I nformation in Land Tenure Reform with Special Reference to Kenya, International Conference on Spatial Information for Sustainable Development, Nairobi, Kenya, 2-5 October, 2001; pp 1-11.

329. Wayumba, G. The Structure of Cadastral System in Kenya. J ournal of Land Administration in Eastern Africa 2017, 1, 5-19.

330. Wayumba, R.; Mwangi, P.; Chege, P. Application of Unmanned Aerial Vehicles in Improving Land Registration in Kenya. International Journal of Research in Engineering and Science (IJ RES) 2017, 5, 7.

331. European Commission. Technology Readiness Levels (Trl). Available online: https://ec.europa.eu/research/participants/data/ref/h2020/wp 2014_2015/annexes/h2020-wp1415-annex-g-trl_en.pdf (accessed on 10 July 2019).

332. Höhle, J. Generating Topographic Map Data from Classification Results. Remote Sensing 2017, 9, 1-24. 
333. Chen, Q.W., Lei; Wu, Yifan; Wu, Guangming; Guo, Zhiling; Waslander, Steven L. Aerial Imagery for Roof Segmentation: A Large-Scale Dataset Towards Automatic Mapping of Buildings. ISPRS J ournal of Photogrammetry and Remote Sensing 2018, 147, 42-55.

334. Volpi, M.; Tuia, D. Deep Multi-Task Learning for a Geographically-Regularized Semantic Segmentation of Aerial Images. ISPRS J ournal of Photogrammetry and Remote Sensing 2018, 144, 48-60.

335. Zhu, X.X.; Tuia, D.; Mou, L.; Xia, G.; Zhang, L.; Xu, F.; Fraundorfer, $F$. Deep Learning in Remote Sensing: A Comprehensive Review and List of Resources. IEEE Geoscience and Remote Sensing Magazine 2017, 5, 8-36.

336. Long, Y.; Gong, Y.; Xiao, Z.; Liu, Q. Accurate Object Localization in Remote Sensing Images Based on Convolutional Neural Networks. IEEE Transactions on Geoscience and Remote Sensing 2017, 55, 2486-2498.

337. University of Auckland. Land Information New Zeeland Data Service. Available online: https://data.linz.govt.nz/ (accessed on 10 October 2019).

338. eurogeographics. Open Cadastral Index Map Service. Available online: https://www.euro-geo-opendata.eu/service/opencadastral-index-map-service (accessed on 10 July 2019).

339. Humanitarian OpenStreetMap Team. Openaerialmap. Available online: https://openaerialmap.org/ (accessed on 10 October 2019).

340. Crommelinck, S.; Koeva, M.; Yang, M.Y.; Vosselman, G. Application of Deep Learning for Delineation of Visible Cadastral Boundaries from Remote Sensing Imagery. Remote Sensing 2019, 11, 1-22.

341. He, H.; Zhou, J.; Chen, M.; Chen, T.; Li, D.; Cheng, P. Building Extraction from UAV Images Jointly Using 6d-SLIC and Multiscale Siamese Convolutional Networks. Remote Sensing 2019, 11, 1-33.

342. Marmanis, D.; Wegner, J.D.; Galliani, S.; Schindler, K.; Datcu, M.; Stilla, U. Semantic Segmentation of Aerial Images with an Ensemble of Cnns. ISPRS Annals of the Photogrammetry, Remote Sensing and Spatial Information Sciences 2016, III-3, 473-480.

343. Yang, M.Y.; Liao, W.; Li, X.; Rosenhahn, B. In Deep Learning for Vehicle Detection in Aerial Images, IEEE International Conference on Image Processing (ICIP), Athens, Greece, 7-10 October, 2018; IEEE: pp 3079-3083.

344. Persello, C.; Tolpekin, V.A.; Bergado, J.R.; de By, R.A. Delineation of Agricultural Fields in Smallholder Farms from 
Satellite Images Using Fully Convolutional Networks and Combinatorial Grouping. Remote Sensing of Environment 2019, 231, 111253.

345. Bergen, K.J.; J ohnson, P.A.; Maarten, V.; Beroza, G.C. Machine Learning for Data-Driven Discovery in Solid Earth Geoscience. Science 2019, 363, 1-10.

346. Ketkar, N. Deep Learning with Python. Apress: 2017; p 169.

347. Garcia-Gasulla, D.; Parés, F.; Vilalta, A.; Moreno, J.; Ayguadé, E.; Labarta, J.; Cortés, U.; Suzumura, T. On the Behavior of Convolutional Nets for Feature Extraction. Journal of Artificial Intelligence Research 2018, 61, 563-592.

348. Stöcker, C.; Ho, S.; Nkerabigwi, P.; Schmidt, C.; Koeva, M.; Bennett, R.; Zevenbergen, J. Unmanned Aerial System Imagery, Land Data and User Needs: A Socio-Technical Assessment in Rwanda. Remote Sensing 2019, 11, 1035.

349. Simonyan, K.; Zisserman, A. Very Deep Convolutional Networks for Large-Scale Image Recognition. arXiv: 1409.1556 2014, 114.

350. He, K.; Zhang, X.; Ren, S.; Sun, J. In Deep Residual Learning for Image Recognition, IEEE Conference on Computer Vision and Pattern Recognition (CVPR), Las Vegas, NV, USA, 26 June1 July, 2016; pp 770-778.

351. Szegedy, C.; Vanhoucke, V.; Ioffe, S.; Shlens, J.; Wojna, Z. In Rethinking the Inception Architecture for Computer Vision, IEEE Conference on Computer Vision and Pattern Recognition (CVPR), Las Vegas, NV, USA, 26 June-1 July, 2016; pp 28182826.

352. Chollet, F. In Xception: Deep Learning with Depthwise Separable Convolutions, IEEE Conference on Computer Vision and Pattern Recognition (CVPR), Honolulu, Hawaii, 21-26 July, 2017; pp 1251-1258.

353. Howard, A.G.; Zhu, M.; Chen, B.; Kalenichenko, D.; Wang, W.; Weyand, T.; Andreetto, M.; Adam, H. Mobilenets: Efficient Convolutional Neural Networks for Mobile Vision Applications. arXiv: 1704.04861 2017, 1-9.

354. Iandola, F.; Moskewicz, M.; Karayev, S.; Girshick, R.; Darrell, T.; Keutzer, K. Densenet: Implementing Efficient Convnet Descriptor Pyramids. arXiv: 1404.1869 2014, 1-14.

355. Fetai, B.; Oštir, K.; Kosmatin Fras, M.; Lisec, A. Extraction of Visible Boundaries for Cadastral Mapping Based on UAV Imagery. Remote Sensing 2019, 11, 1510.

356. Deng, J.; Dong, W.; Socher, R.; Li, L.-J.; Li, K.; Fei-Fei, L. In Imagenet: A Large-Scale Hierarchical Image Database, IEEE Conference on Computer Vision and Pattern Recognition (CVPR), Miami Beach, FL, USA, 20-25 June, 2009; pp 248-255. 
357. Chollet, F. Keras. Available online: https://keras.io (accessed on 10 October 2019).

358. Kohli, D.; Bennett, R.; Lemmen, C.; Asiama, K.; Zevenbergen, J . In A Quantitative Comparison of Completely Visible Cadastral Parcels Using Satellite Images: A Step Towards Automation, FIG Working Week, Helsinki, Finland, 29 May-2 June, 2017; pp 1-14.

359. Fang, L.; Cunefare, D.; Wang, C.; Guymer, R.H.; Li, S.; Farsiu, S. Automatic Segmentation of Nine Retinal Layer Boundaries in Oct Images of Non-Exudative Amd Patients Using Deep Learning and Graph Search. Biomedical Optics Express 2017, 8, 2732-2744.

360. Long, J.; Shelhamer, E.; Darrell, T. In Fully Convolutional Networks for Semantic Segmentation, IEEE Conference on Computer Vision and Pattern Recognition (CVPR), Boston, MA, USA, 7-12 June, 2015; pp 3431-3440.

361. Yang, J.; Price, B.; Cohen, S.; Lee, H.; Yang, M.-H. In Object Contour Detection with a Fully Convolutional Encoder-Decoder Network, IEEE Conference on Computer Vision and Pattern Recognition (CVPR), Las Vegas, NV, USA, 26 June-1 July, 2016; pp 193-202.

362. Li, P.; Zang, Y.; Wang, C.; Li, J.; Cheng, M.; Luo, L.; Yu, Y. In Road Network Extraction Via Deep Learning and Line Integral Convolution, IEEE International Geoscience and Remote Sensing Symposium (IGARSS), Beijing, China, 10-15 July, 2016; pp 1599-1602.

363. Zhou, W.; Wu, C.; Yi, Y.; Du, W. Automatic Detection of Exudates in Digital Color Fundus Images Using Superpixel MultiFeature Classification. IEEE Access 2017, 5, 17077-17088.

364. Butenuth, M.; Heipke, C. Network Snakes: Graph-Based Object Delineation with Active Contour Models. Machine Vision and Applications 2012, 23, 91-109.

365. Gerke, M.; Butenuth, M.; Heipke, C.; Willrich, F. GraphSupported Verification of Road Databases. ISPRS Journal of Photogrammetry and Remote Sensing 2004, 58, 152-165.

366. Lucas, C.; Bouten, W.; Koma, Z.; Kissling, W.D.; Seijmonsbergen, A.C. Identification of Linear Vegetation Elements in a Rural Landscape Using Lidar Point Clouds. Remote Sensing 2019, 11, 1-16.

367. Nyandwi, E.; Koeva, M.; Kohli, D.; Bennett, R. Comparing Human Versus Machine-Driven Cadastral Boundary Feature Extraction. Preprints 2019, 2019050342.

368. Warner, T.A.; Fang, F. Implementation of Machine-Learning Classification in Remote Sensing: An Applied Review. International J ournal of Remote Sensing 2018, 39, 2784-2817. 
369. Rottensteiner, F.; Sohn, G.; Jung, J.; Gerke, M.; Baillard, C.; Benitez, S.; Breitkopf, U. The ISPRS Benchmark on Urban Object Classification and 3D Building Reconstruction. ISPRS Annals of the Photogrammetry, Remote Sensing and Spatial Information Sciences 2012, I-3, 293-298.

370. Demir, I.; Koperski, K.; Lindenbaum, D.; Pang, G.; Huang, J.; Basu, S.; Hughes, F.; Tuia, D.; Raskar, R. In Deepglobe 2018: A Challenge to Parse the Earth through Satellite Images, IEEE/CVF Conference on Computer Vision and Pattern Recognition Workshops (CVPRW) Salt Lake City, UT, USA, 1822 June, 2018; pp 172-181.

371. Cardim, G.; Silva, E.; Dias, M.; Bravo, I.; Gardel, A. Statistical Evaluation and Analysis of Road Extraction Methodologies Using a Unique Dataset from Remote Sensing. Remote Sensing 2018, 10, 1-17.

372. Debats S. R.; Estes L. D. ; Thompson D. R.; K., C.K. Integrating Active Learning and Crowdsourcing into Large-Scale Supervised Landcover Mapping Algorithms. PeerJ Preprints 2017, 5:e3004v1, 1-26.

373. Keenja, E.; De Vries, W.; Bennett, R.; Laarakker, P. In Crowd Sourcing for Land Administration: Perceptions within Netherlands Kadaster, FIG Working Week, Rome, Italy, 6-10 May, 2012; pp 1-12.

374. Basiouka, S.; Potsiou, C. Vgi in Cadastre: A Greek Experiment to Investigate the Potential of Crowd Sourcing Techniques in Cadastral Mapping. Survey Review 2012, 44, 153-161.

375. Moreri, K.; Fairbairn, D.; James, P. Issues in Developing a Fit for Purpose System for Incorporating Vgi in Land Administration in Botswana. Land Use Policy 2018, 77, 402-411.

376. Spatial Collective. Mapping: (No) Big Deal. Available online: http://mappingnobigdeal.com/ (accessed on 10 October 2019).

377. Crommelinck, S. Delineation-Tool Wiki. Available online: https://github.com/its4land/delineation-tool/wiki (accessed on 10 October 2019).

378. Koeva, M.; Bennett, R.; Gerke, M.; Crommelinck, S.; Stöcker, C.; Crompvoets, J.; S. Ho; Schwering, A.; Chipofya, M.; Schultz, C., et al. In Towards Innovative Geospatial Tools for Fit-forPurpose Land Rights Mapping, ISPRS Geospatial Week, Wuhan, China, 18-22 September, 2017; XLII-2/W, pp 37-43.

379. Gerke, M.; Stöcker, C.; Crommelinck, S.; Koeva, M. UAV Für Das Kadaster - Das Eu-Projekt Its4land. In UAV 2018 Vermessung Mit Unbemannten Flugsystemen: Beiträge Zum 169. Dvw-Seminar, Deutsche Gesellschaft fur Geodesie, Geoinformation, und Landmanagement (DVW): 2018; Vol. 89, pp 105-116. 
380. Kohli, D.; Crommelinck, S.; Bennett, R.; Koeva, M.; Lemmen, C. In Object-Based Image Analysis Methods for Cadastral Boundary Mapping Using Satellite Images, Image and Signal Processing for Remote Sensing XXIII, Warsaw, Poland, 4 October 2017, 2017; 10427.

381. Crommelinck, S.; Koeva, M. Towards Cadastral Intelligence? GIM International 2019, 27-29.

382. Marshall, M.; Crommelinck, S.; Kohli, D.; Perger, C.; Yang, M.Y.; Ghosh, A.; Fritz, S.; Bie, K.d.; Nelson, A. Crowd-Driven and Automated Mapping of Field Boundaries in Highly Fragmented Agricultural Landscapes of Ethiopia with Very High Spatial Resolution Imagery. Remote Sensing 2019, 11, 2082.

All data used for this research have been published through Data Archiving and Networked Services (DANS) on behalf of the Royal Netherlands Academy of Arts and Sciences. DOI: 10.17026/dans-24k-fxw4 


\section{Summary}

Recording land rights provides land owners tenure security, sustainable livelihood and increases financial opportunities. Estimates suggest that about $75 \%$ of the world population does not have access to a formal system to register and safeguard their land rights. This lack of recorded land rights increases insecure land tenure and fosters existence-threatening conflicts, particularly in developing countries. Recording land rights spatially is defined as cadastral mapping or cadastral boundary delineation. Cadastral boundaries can either be recorded on the ground with accurate measurement equipment (direct surveying) or be delineated along visible boundaries from remote sensing imagery (indirect surveying). Cadastral mapping is considered the most expensive part of a land administration system.

Recent developments in technology allow us to rethink contemporary cadastral mapping. Unmanned aerial vehicles (UAVs) known as drones are evolving as an alternative tool to acquire land tenure data. UAVs can capture geospatial data at high-resolution in a low-cost, transparent, and flexible manner. Imagery captured with UAVs is increasingly used in indirect surveying to accurately delineate visible cadastral boundaries. Many cadastral boundaries are visible, as they are demarcated by physical objects such as fences, walls, roads, buildings, or rivers. Furthermore, recent advances in automated detection and localization of objects from images offer new opportunities for indirect surveying: instead of delineating visible boundaries manually from lowresolution imagery, boundaries can be extracted automatically by applying image analysis to high-resolution UAV imagery. Such solutions offer the potential to improve current cadastral mapping procedures in terms of time, cost, and accuracy for the sake of worldwide land tenure security and sustainable land administration.

This Ph.D. research introduces an approach that simplifies image-based cadastral mapping. We develop an automated cadastral boundary delineation approach that is applicable to remote sensing data of high-resolution. The approach is designed for areas, in which boundaries are demarcated by physical objects and are thus visible. Areas of investigation are East African developing countries including Kenya, Rwanda, and Ethiopia.

In chapters 1 and 3, we review the state-of-the-art on cadastral mapping, boundary delineation, UAV photogrammetry, feature extraction, as well as their interactions. The review reveals that automating indirect surveying from UAV data is a recently emerging research field. In practice, indirect surveying from UAV or any other remotely sensed image appears to be rarely automated and relies mostly on manual delineation through on-screen delineation. We show the potential of automated UAV-based cadastral mapping. The review 
covers feature extraction methods that are synthesized into a hypothetical workflow consisting of image segmentation, line extraction, and contour generation.

In chapters 4, 5 and 6, the hypothetical workflow is implemented step by step by testing and adapting previously reviewed methods. Our results show that methods from computer vision are suitable to precisely extract object outlines demarcating cadastral boundaries. However, the methods are developed to work well on small images and not necessarily on UAV images of many more pixels. We adapt these computer vision methods and apply them to remote sensing data that cover large-scale imagery and 3D information. For image segmentation, we find that Globalized Probability of Boundary ( $\mathrm{gPb}$ ) contour detection extracts objects at completeness and correctness rates of up to $80 \%$ (chapter 4). For line extraction, we find that Simple Linear Iterative Clustering (SLIC) delineates the objects with the high accuracy provided by the UAV imagery at completeness rates of up to $64 \%$ (chapter 5). For contour generation, we implement machine learning through Random Forest (RF) classification to combine the results of $\mathrm{gPb}$ and SLIC. Further, we develop a procedure for a subsequent interactive delineation. Compared to manual delineation, the number of clicks per $100 \mathrm{~m}$ is reduced by up to $86 \%$, while obtaining a similar localization quality (chapter 6).

In chapters 7 and 8, the approach is optimized and evaluated for cadastral mapping. While our previous evaluations use objects such as roads and buildings that potentially demarcate cadastral boundaries, the evaluations in chapter 7 and 8 compare results to the intended end product: cadastral boundaries. Furthermore, potential end users are involved and asked for feedback. The workflow's complexity is reduced by replacing gPb and SLIC with another computer vision method, namely Multiscale Combinatorial Grouping (MCG) (chapter 7). The accuracy of boundary likelihoods predicted by machine learning is improved by $11 \%$. The degree of automation is increased by replacing RF classification with deep learning Convolutional Neural Networks (CNN) (chapter 8). The final workflow consists of image segmentation, boundary classification, and interactive delineation. The workflow is tested on UAV and aerial imagery. We show that our approach is less tiring and more effective in terms of clicks and time compared to manual delineation for parcels surrounded by visible boundaries. Strongest advantages are obtained for rural scenes delineated from aerial imagery, where the delineation effort per parcel is requires $38 \%$ less time and $80 \%$ fewer clicks compared to manual delineation.

We aimed to automate the extraction of cadastral boundaries by translating the intelligence of a human delineator into a machine learning approach. So far, our workflow based on deep learning has not been shown to be 'intelligent' 
enough to replace a human delineator. Cadastral intelligence, which we define as deep learning frameworks for cadastral boundary delineation, requires further improvements before fully replacing manual delineation. However, we show cases where our approach is already superior to current manual delineation practices.

A successful application of our approach in a real-world use case requires more cycles of designing, developing, testing, and evaluating. This demands analyses beyond accuracy and efficiency that incorporate existing social, legal, and institutional frameworks, as well as further method development. One major limitation impeding method development throughout the Ph.D. research was the unavailability of UAV and cadastral data covering areas with many visible boundaries.

Overall, this manuscript illustrates how state-of-the-art knowledge from remote sensing, geo-informatics, photogrammetry, computer vision, and machine learning can be combined into an innovative cadastral mapping approach. By making use of synergies, we developed an approach that is superior to manual delineation when object outlines are continuously visible and coincide with cadastral boundaries. In future work, it can be adapted and transferred to real-world cadastral mapping use cases. The automated approach simplifies and speeds-up the delineation of objects from imagery. While the approach has been developed for cadastral mapping, it can also be used to delineate objects in other application fields, such as land use mapping, topographical mapping, road tracking, or building extraction. This Ph.D. research can be considered an innovative impulse for improving manual delineation in land administration and beyond. 


\section{Samenvatting}

Het optekenen van eigendomsrechten geeft aan grondbezitters naast de zekerheid van recht op eigendom, tevens een uitzicht op financiële kansen. Uit schattingen blijkt dat bijna 75\% van de wereldbevolking nog steeds geen toegang heeft tot formeel aanvaarde landregistratiesystemen. Het ontbreken van kadastraal geregistreerde landrechten geeft aanleiding tot onzekerheid omtrent landeigendom en hierdoor soms tot levensbedreigende conflicten. Het optekenen of registreren van lokale landrechten wordt kadastrale kartering of kadastrale grensafbakening genoemd. Kadastrale grenzen kunnen op het terrein worden vastgelegd met behulp van nauwkeurige meetapparatuur (de zogenaamde directe meting). Zichtbare grenzen kunnen ook worden gedigitaliseerd vanuit luchtbeelden via telemetrie (indirecte metingen). Veel kadastrale grenzen zijn zichtbaar, omdat ze worden afgebakend door objecten zoals hekkens, muren, wegen, gebouwen of rivieren. Omdat de opmaak van kadastrale kaarten vaak het duurste deel van een landadministratiesysteem is, zijn nieuwe benaderingen nodig die een snelle, nauwkeurige en kostenefficiënte afbakening van de eigendomsgrenzen mogelijk maken. Dit zal leiden tot meer eigendoms-rechtszekerheid en tot een meer duurzaam beleid.

Recente technologische ontwikkelingen maken het mogelijk om de opmaak van de hedendaagse kadastrale kaarten opnieuw te bezien. Onbemande luchtvoertuigen (UAV) evolueren tot een alternatief instrument om gegevens over grondbezit te verwerven. UAV's kunnen ruimtelijke gegevens van hoge kwaliteit en resolutie op een kostenefficiënte, transparante en flexibele manier vastleggen, van waaruit dan zichtbare perceelsgrenzen kunnen onderscheiden worden. Bovendien maken verbeteringen in geautomatiseerde detectie- en lokalisatie-methoden van objecten uit afbeeldingen, een heroverweging van het gebruik van indirecte metingen mogelijk: in plaats van het handmatig afbakenen van zichtbare grenzen van afbeeldingen met lage resolutie, kunnen nu grenzen automatisch worden geëxtraheerd door beeldanalysetechnieken te koppelen aan beschikbare UAV-gegevens met hoge resolutie. Een dergelijke oplossing biedt de mogelijkheid om de huidige kadastrale karteringsaanpak te verbeteren zowel in betrekking tot tijd, kosten en nauwkeurigheid van de resultaten.

Dit doctoraatsproefschrift licht de aanpak toe, die het maken van beeldgebaseerde kadastrale kaarten vergemakkelijkt voor het automatisch in kaart brengen van eigendomsgrenzen. We ontwikkelen een open-source systeem van geautomatiseerde belijning van kadastrale grenzen dat toepasbaar is op UAV-beelden. Het systeem is ontworpen voor gebieden, waar grenzen worden afgebakend door fysieke objecten en dus zichtbaar zijn. Toepassingsgebieden zijn Oost-Afrikaanse landen, waaronder Kenia, Rwanda en Ethiopië. In de loop 
van dit doctoraal onderzoek hebben we onze innovatieve aanpak van kadastrale kartering verder ontwikkeld, geëvalueerd en verfijnd.

In de hoofdstukken 1 en 3 bespreken we de aan dit onderwerp gerelateerde informatie. We beoordelen het nieuwste-van-het-nieuwste in verband met onder meer kadastrale kartering, afbakening van grenzen, UAVfotogrammetrie en functie-extractie, evenals combinaties van de diverse systemen. Uit dit onderzoek blijkt dat het automatiseren van indirecte terreinverkenning vanuit UAV-gegevens nog een nauwelijks bestaand onderzoeksgebied is. In de praktijk lijken indirecte metingen via UAV of andere beelden die via telemetrie worden verkregen, zelden te worden geautomatiseerd. De opmaak van de kadastrale begrenzingen berust grotendeels op een handmatige afbakening via digitalisering van het beeld op het scherm. We belichten het potentieel van geautomatiseerde op UAV gebaseerde kadastrale kaarten. Dit proefschrift behandelt diverse mogelijkheden, de zogenaamde "feature-extractiemethoden" die samen komen tot een hypothetische workflow die bestaat uit zowel beeldsegmentatie, lijnextractie, als het genereren van objectomtrekken. Deze workflow wordt stap voor stap geïmplementeerd in de volgende hoofdstukken.

In de hoofdstukken 4, 5 en 6 wordt een geschikte aanpak ontwikkeld door eerder beoordeelde methoden in de praktijk te testen en aan te passen. Onze resultaten laten zien dat methoden gebaseerd op computertechnieken geschikt zijn om nauwkeurig objectomtrekken af te leiden die de kadastrale grenzen op het terrein afbakenen. De methoden zijn echter ontwikkeld om goed te werken op basis van kleine afbeeldingen en niet noodzakelijk op UAV-afbeeldingen die veel meer pixels bevatten. We zetten via gecomputeriseerde visualisatiemethoden de gegevens om, teneinde resultaten te verkrijgen uit telemetrie die zowel beelden als 3D informatie bevatten. Bij het opdelen van beelden in diverse segmenten, kunnen we door het gebruik van veralgemeende programmatuur gebaseerd op waarschijnlijkheid van grensbepaling van omtrekken of contouren $(\mathrm{gPb}=$ globalised probability boundery), in uitbreiding, de omtrekken van de objecten met een nauwkeurigheid en volledigheid van maximaal $80 \%$ verkrijgen (hoofdstuk 4). Voor het ontrafelen van lijnvormige afbakeningen hebben we gevonden dat Simple Linear I terative Clustering (SLIC) de objecten afbeeldt, met de hoge nauwkeurigheid van de UAV-ortho-afbeeldingen, met een ratio naar volledigheid van maximaal $64 \%$ (hoofdstuk 5). Voor het genereren van object-contouren passen we artificiële intelligentie toe door gebruik te maken van de Random Forest (RF) classificatie, die de resultaten van gPb en SLIC combineert. Verder ontwikkelen we een procedure voor een volgende interactieve afbakening. In vergelijking met handmatige afbakening wordt het aantal klikken per 100 strekkende meter lijn-lengte met maximaal $86 \%$ verminderd, terwijl een vergelijkbare plaatsbepalingskwaliteit wordt verkregen (hoofdstuk 6). 
In de hoofdstukken 7 en 8 wordt de aanpak voor de opmaak van kadastrale kaarten geoptimaliseerd en geëvalueerd. Terwijl onze vorige evaluaties objecten gebruikten die mogelijk kadastrale grenzen afbakenden, zoals wegen en gebouwen, vergelijken de evaluaties beschreven in hoofdstukken 7 en 8 , nu de verkregen resultaten met het beoogde eindproduct: kadastrale grenzen van de eigendommen vastleggen. Verder zijn potentiële eindgebruikers betrokken en gevraagd om feedback te geven. De complexiteit van de workflow wordt verminderd door $\mathrm{gPb}$ en SLIC te vervangen door een andere gecomputeriseerde benadering, namelijk de methode van de zogenaamde Multiscale Combinatorial Grouping (MCG) (hoofdstuk 7). De juistheid van de voorspelde objectgrenzen wordt hierdoor met $11 \%$ verbeterd en de mate van automatisering wordt verhoogd door de RF-classificatie te vervangen door de zogenaamde deep learning Convolutional Neural Networks (CNN) (hoofdstuk 8). De uiteindelijke workflow bestaat uit achtereenvolgens beeldontrafeling, classificatie van de soorten grenzen en een interactieve belijning van de contouren van het object. Deze aanpak is getest op gegevens afkomstig van UAV en van luchtfoto's. We laten zien dat onze aanpak minder arbeidsintensief en efficiënter is wat betreft het aantal benodigde klikken en de vereiste tijdsspanne in vergelijking met de handmatige afbakening voor percelen die omgeven zijn door zichtbare grenzen. De belangrijkste voordelen van deze werkwijze worden verkregen voor landelijke omgevingen die zijn afgeleid van luchtfoto's, waarbij de benodigde afbakenings-inspanning ca. $38 \%$ minder tijd vergt en $80 \%$ minder klikken per perceel.

We wilden de extractie van kadastrale grenzen automatiseren door de intelligentie van een menselijke cartograaf van kadastrale grenzen te vertalen in een opmaak van een algoritme voor een zelflerende aanpak. Onze workflow, op basis van machine learning is tot dusver niet 'intelligent' genoeg gebleken om een menselijke landmeter te vervangen. Kadastrale intelligentie, die we definiëren als de opmaak van een verdiepend lerend werk-kader voor de afbakening van kadastrale grenzen, vereist verdere verbeteringen voordat de handmatige afbakening door een menselijke cartograaf wordt vervangen. Nochtans lichten we in dit proefschrift toch gevallen toe waarvoor onze aanpak voornamelijk naar precisie, snelheid en accuraatheid, al superieur is aan de huidige handmatige afbakeningspraktijken.

Een succesvolle toepassing van de aanpak aan één specifiek scenario vraagt verdere inspanningen en vereist meer cycli van ontwerp, ontwikkeling, testen en evaluatie. Dit vereist analyses die de beoogde graad van nauwkeurigheid en efficiëntie overstijgen en die bestaande sociale, wettelijke en institutionele kaders en verdere methodeontwikkeling omvatten. Een belangrijke beperking die de ontwikkeling van methoden tijdens het doctoraatsonderzoek belemmerde was de ontbrekende beschikbaarheid van UAV en kadastrale gegevens over gebieden met veel zichtbare grenzen. 
Tenslotte kunnen we stellen dat dit proefschrift illustreert hoe de "state-of-theart" kennis van telemetrie, geo-informatica, fotogrammetrie, computer vision, en artificiële intelligentie kan worden gekoppeld aan een innovatieve aanpak van kadastrale kartering. Door gebruik te maken van synergiën hebben we de mogelijkheid om een benadering van de kartering en afbakening te ontwikkelen die superieur is ten opzichte van de huidige handmatige afbakeningspraktijken, en dit in gebieden waarin objectcontouren continu zichtbaar zijn en samenvallen met kadastrale grenzen.

In de toekomst, kan de werkwijze nog worden aangepast en omgezet tot realworld toepassingen van kadastrale kartering. De geautomatiseerde benadering vereenvoudigt en versnelt de bepaling van de belijning van objecten afkomstig van beeldmateriaal. Terwijl deze innovatieve benadering in de eerste plaats is ontwikkeld voor kadastrale kartering, kan deze aanpak ook haar toepassing vinden in het digitaliseren van objecten ten behoeve van andere domeinen zoals bijvoorbeeld het karteren van percelen voor divers gebruik, topografische kartering, wegtracering of herkenning van bouwwerken. 


\section{Author's Biography}

Sophie Crommelinck was born on May $8^{\text {th }}$ 1992 in Cologne (Germany). A daughter of Belgian parents, she has the Belgian nationality. Sophie grew up in Aachen (Germany), where she graduated from the bilingual French-German class at the Rhine-Maas-Gymnasium in 2011. At age 16, she spent an exchange year at the Rutherford High School in New J ersey

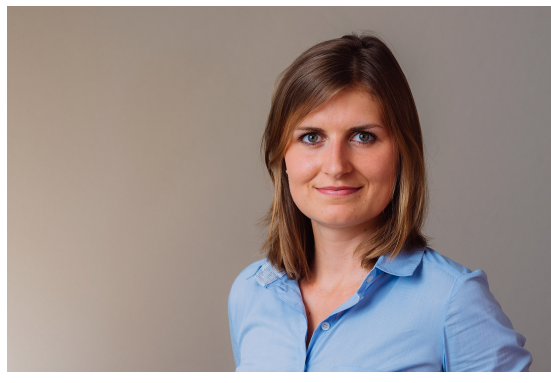
(US). In 2011, she moved to Heidelberg (Germany) to study Geography at the Heidelberg University. During her studies, she spent one semester at the University Paul Valéry in Montpellier (France). After finishing her B.Sc. in 2014, she continued her studies at Heidelberg University to obtain a M.Sc. in Geography with a specialization in Geo-Informatics. With her M.Sc. thesis, she was nominated for the Karl Kraus Young Scientists Award issued by the German Society for Photogrammetry, Remote Sensing and Geo-information.

In February 2016, she started her Ph.D. research at the Faculty of GeoInformation Science and Earth Observation (ITC) of the University of Twente in Enschede (Netherlands). The present manuscript is the result of this research. In 2017, her first paper was selected as cover story of a leading remote sensing journal. In the same year, she spent five months as a guest researcher in the GIScience research group at Heidelberg University. As part of her Ph.D., she attended the following events to present and discuss her research:

\begin{tabular}{|c|c|c|}
\hline Date & Event & Place \\
\hline 23.-26. August 2016 & FOSS4G & Bonn, Germany \\
\hline 14.-16. September 2016 & GEOBIA & Enschede, Netherlands \\
\hline 06.-09. J une 2017 & ISPRS Workshop & Hannover, Germany \\
\hline 28. June 2017 & FOSS4G NL & Groningen, Netherlands \\
\hline 29.-30. June 2017 & LANDac & Utrecht, Netherlands \\
\hline 02.-11. August 2017 & QGIS Developer Meeting & Nødebo, Denmark \\
\hline 04.-07. September 2017 & UAV-g & Bonn, Germany \\
\hline 24. November 2017 & $\begin{array}{l}\text { Fachaustausch } \\
\text { Geoinformation }\end{array}$ & Heidelberg, Germany \\
\hline 04.-07. June 2018 & ISPRS TC II Symposium & Riva del Garda, I taly \\
\hline 13.-14. June 2018 & Urban Studies Symposium & Enschede, Netherlands \\
\hline 11. July 2018 & FOSS4G NL & Almere, Netherlands \\
\hline $\begin{array}{l}\text { 28. September - } \\
02 \text {. October } 2018\end{array}$ & its4land Workshop & Kajiado \& Nairobi, Kenya \\
\hline 25. October 2018 & $\begin{array}{l}\text { Symposium on Feature } \\
\text { Extraction for Cadastral } \\
\text { Boundaries }\end{array}$ & Enschede, Netherlands \\
\hline 29. November 2018 & NCG Symposium & Wageningen, Netherlands \\
\hline 03. April 2019 & Geospatial World Forum & Amsterdam, Netherlands \\
\hline
\end{tabular}




\begin{tabular}{rll}
\hline 10.-14. June 2019 & Geospatial Week & Enschede, Netherlands \\
\hline 23.-24. July 2019 & EALAN Conference & Zanzibar, Tanzania \\
\hline 13.-16. August 2019 & AfriGEOSS Symposium & Nairobi, Kenya \\
\hline
\end{tabular}

Towards the end of her Ph.D. research, she was an invited reviewer for scientific manuscripts submitted to Remote Sensing, ISPRS Annals, Land Use Policy, International Journal of Remote Sensing, and the Conference on Computer Vision and Pattern Recognition. She was part of the scientific committee for the 3D Geolnfo conference in 2018 and 2019.

She attended courses for 52 ECTS from the Twente Graduate School and further institutions on personal development and technical expertise. During her Ph.D., she was involved in teaching the two courses Scientific Geocomputing and Land Administration at the ITC. Further, she was a coach in the Academic Publishing Bootcamp of the Twente Graduate School.

During her Ph.D. research she authored and co-authored the following publications:

Crommelinck, S.; Bennett, R.; Gerke, M.; Nex, F.; Yang, M.Y.; Vosselman, G. Review of Automatic Feature Extraction from High-Resolution Optical Sensor Data for UAV-Based Cadastral Mapping. Remote Sensing 2016, 8, 1-28.

Crommelinck, S.; Bennett, R.; Gerke, M.; Koeva, M.; Yang, M.Y.; Vosselman, G. In SLIC Superpixels for Object Delineation from UAV Data, International Conference on Unmanned Aerial Vehicles in Geomatics, Bonn, Germany, 04-07 September, 2017; IV-2/W3, pp 9-16.

Crommelinck, S.; Bennett, R.; Gerke, M.; Yang, M.Y.; Vosselman, G. Contour Detection for UAV-Based Cadastral Mapping. Remote Sensing 2017, 9, 1-13.

Crommelinck, S.; Höfle, B.; Koeva, M.; Yang, M.Y.; Vosselman, G. Interactive Boundary Delineation from UAV Data. ISPRS Annals of the Photogrammetry, Remote Sensing and Spatial Information Sciences 2018, IV-2, 81-88.

Crommelinck, S.; Koeva, M.; Yang, M.Y.; Vosselman, G. Application of Deep Learning for Delineation of Visible Cadastral Boundaries from Remote Sensing Imagery. Remote Sensing 2019, 11, 22.

Crommelinck, S.; Koeva, M. Towards Cadastral Intelligence? GI M International 2019, 27-29. 
Bennett, R.; Gerke, M.; Crompvoets, J.; Ho, S.; Schwering, A.; Chipofya, M.; Schultz, C.; Zein, T.; Biraro, M.; Alemie, B., et al. In Building Third Generation Land Tools: Its4land, Smart Sketchmaps, UAVs, Automatic Feature Extraction, and the Geocloud, World Bank Conference on Land and Poverty, Washington, DC, USA, 20-24 March, 2017; pp 1-23.

Koeva, M.; Bennett, R.; Gerke, M.; Crommelinck, S.; Stöcker, C.; Crompvoets, J.; S.Ho; Schwering, A.; Chipofya, M.; Schultz, C., et al. In Towards Innovative Geospatial Tools for Fit-for-Purpose Land Rights Mapping, ISPRS Geospatial Week, Wuhan, China, 18-22 September, 2017; XLII2/W, pp 37-43

Kohli, D.; Crommelinck, S.; Bennett, R.; Koeva, M.; Lemmen, C. In ObjectBased Image Analysis Methods for Cadastral Boundary Mapping Using Satellite Images, Image and Signal Processing for Remote Sensing XXIII, Warsaw, Poland, 4 October 2017, 2017; 10427.

Gerke, M.; Stöcker, C.; Crommelinck, S.; Koeva, M. UAV Für Das Kadaster Das Eu-Projekt Its4land. In UAV 2018 - Vermessung Mit Unbemannten Flugsystemen: Beiträge Zum 169. Dvw-Seminar, Deutsche Gesellschaft fur Geodesie, Geoinformation, und Landmanagement (DVW): 2018; Vol. 89, pp 105-116.

Koeva, M.; Crommelinck, S.; Stöcker, C.; Crompvoets, J.; Ho, S.; Buntix, I.; Schwering, A.; Chipofya, M.; Sahib, J.; Zein, T., et al. In Its4land Challenges and Opportunities in Developing Innovative Geospatial Tools for Fit-for-Purpose Land Rights Mapping, FIG Congress, Istanbul, Turkey, 6-11 May, 2018; pp 1-17.

Marshall, M.; Crommelinck, S.; Kohli, D.; Perger, C.; Yang, M.Y.; Ghosh, A.; Fritz, S.; Bie, K.d.; Nelson, A. Crowd-Driven and Automated Mapping of Field Boundaries in Highly Fragmented Agricultural Landscapes of Ethiopia with Very High Spatial Resolution Imagery. Remote Sensing 2019, 11, 2082. 